\title{
A Measurement of the Lifetime of the $\Lambda_{b}$ Baryon with the CDF Detector at the Tevatron Run II
}

\author{
Tatjana Alberta Hanna Unverhau
}

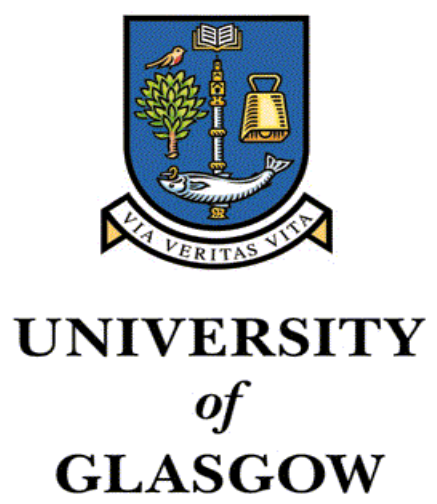

December 2004

A thesis submitted for the degree of Doctor of Philosophy of the University of Glasgow

(C)Tatjana Unverhau 2004 
To my parents and John, my husband, to my grandfather Hans whom I would have loved to know and to my grandfather "Opa" who would have loved to see this 


\section{Abstract}

In March 2001 the Tevatron accelerator entered its Run II phase, providing colliding proton and anti-proton beams with an unprecedented centre-of-mass energy of $1.96 \mathrm{TeV}$. The Tevatron is currently the only accelerator to produce $\Lambda_{b}$ baryons, which provides a unique opportunity to measure the properties of these particles. This thesis presents a measurement of the mean lifetime of the $\Lambda_{b}$ baryon in the semileptonic channel $\Lambda_{b}^{0} \rightarrow \Lambda_{c}^{+} \mu^{-} \bar{\nu}_{\mu}$. In total $186 \mathrm{pb}^{-1}$ of data were used for this analysis, collected with the CDF detector between February 2002 and September 2003. To select the long-lived events from b-decays, the secondary vertex trigger was utilised. This significant addition to the trigger for Run II allows, for the first time, the selection of events with tracks displaced from the primary interaction vertex at the second trigger level. After the application of selection cuts this trigger sample contains approximately $991 \Lambda_{b}$ candidates. To extract the mean lifetime of $\Lambda_{b}$ baryons from this sample, the transverse decay length of the candidates is fitted with an unbinned maximum likelihood fit under consideration of the missing neutrino momentum and the bias introduced by the secondary vertex trigger. The mean lifetime of the $\Lambda_{b}$ is measured to be

$$
\tau=1.29 \pm 0.11 \text { (stat.) } \pm 0.07 \text { (syst.) ps }
$$

equivalent to a mean decay length of

$$
\mathrm{c} \tau=387 \pm 33 \text { (stat.) } \pm 21 \text { (syst.) } \mu \mathrm{m} \text {. }
$$




\section{Acknowledgements}

Why is it that acknowledgements seem to be almost the hardest part to write (apart from the fact that its spelling is not straight forward for a "foreigner")? I am not saying that writing the rest of this thesis was easy - on the contrary, and it has taken long enough, but the major part of a thesis is still the work that goes into obtaining the results. Bringing them down to paper is not that simple, but there are kind spirits that read through drafts, suggest different wording or an extra figure. But who tells you that you have forgotten to thank a person that has made life more worthwhile or work better during the years of the Ph.D.? Well, nobody.

While a lot of people helped me during those good four years of my thesis in one way or another they will not speak up to safe their place in my acknowledgements because that is not how it works and that is exactly why acknowledgements are so hard to write! So here are my preemptive apologies to those I should have mentioned but forgot.

A thesis starts with funding and I am most grateful to the University of Glasgow which provided me through a University Scholarship with the opportunity to come to Glasgow and work on the CDF experiment. Glasgow is a fantastic place and one of the very few cities where I felt home. Obviously I missed people back home, but the feel of the city was right (I should mention though that I was lucky to land in the West End). I need to thank Ken, because he planted the idea of coming to Glasgow in my head, and who could be better suited to convince you that Glasgow is a place with nice people. On the funding side of things I also want to thank David Saxon and Rick St. Denis and the people organising the McRobertson scholarship for making sure I could spend time at Fermilab near Chicago where the experiment 
is situated. When my funded time had run out the money Onkelchen had left me helped a great deal and I hope he finds that a worthwhile use of his legacy. Thank you Onkelchen.

Then of course a topic is rather essential, and here I thank my supervisor Peter Bussey and Marjorie Shapiro for their help in finding an "unoccupied" topic in my second year. At Fermilab there is a large number of very helpful people I am indebted to for the one or other important information, the kind of information that can save weeks or months of work if it is available. Amongst those are Hung-Chung Fang, Shin-Shan Yu, Will, Rick T., Guillelmo, Andreas Korn, Konstantin, Christoph, Thilo, Jonathan Lewis, Saverio, Stefano, Alex Cerri, Robin and several others. I am most grateful however for the help of two people that don't seem to sleep: Masashi Tanaka and Satoru Uozumi. In many discussions their opinion and knowledge has been a great deal of help. That is another thing about acknowledgements - you thank people, but it is hard to bring across just how helpful and patient and important for the progress of a thesis some people have been. So thanks again to Satoru and Masa.

In Glasgow I want to thank Alan Flavell and David Martin for their support. Alan sat many a night in the department helping to set up a meeting scheduled late at night. Thanks to Mahfuzur for saving me a space in the "fish"-office, which had brilliant office mates - Debby, Chris, Francesco, James - and a big fish tank. Big big thanks go to Stan - not just for the social aspects of things and his vast knowledge of real ales and even more impressive knowledge of small German breweries that shadows mine. Knowing that Stan is in the department, you know that things cannot be so bad; talk to Stan and he will try his best to help - whether that is a last minute read through the second year report, finding a PC that works, or office space, you name it. Stan, like Peter and John also deserve thanks for reading through the drafts of my thesis. John in particular had to suffer because he was most readily available and had to answer countless questions of the kind: "where do you think the comma should go here?". Thanks (and sorry).

But now to the social side of things. Richard and Alison have been fantastic friends throughout the whole time, including a little survival bag for the life across the ocean, Ermentrude and monkey, the lovely painting and the dragon of course! 
Jumping to Fermilab the first person I need to thank is Tim. When I arrived at Fermilab Tim was away for a while, but had left the keys to his car - a red Buick. A car is a must over there and it helped a lot. But of course the car is the least of things he deserves thanks for. He has always been a true friend and for that I thank him. Thanks to Fiona for the company on walks with their adoptive German shepherd (which were fun, honest, even with all the ticks we brought back) and for her help to find a car for myself.

The number of fellow students from the U.K. that made Fermilab, Glasgow and also the RAL summer school more fun is rather large. I just pick a few here I have spent the most time with (particularly the non-fermi-folks may forgive me). Thanks to Helen, Anant, Dustin, Bryan, Simon \& Simon (D0Si), Martin, Lucio, Kyle, Gavin, Tamsin, Emily and Liddy. Thanks to Dustin et. al. for spontaneous cinema nights. Thanks to D0Si for letting me stay in his flat, for generally being good fun and for his humour. Somehow we didn't see so much of each other as D0Si works rather odd hours, but on occasion we managed. And thanks to his lovely wife Farah and to Joana for the fun during the summer school. Thanks to Simon for tons of things. He has been a great friend and made the time at Fermilab so much better. Thanks for the great trips to see thousands of Sandhill Cranes, for sarcastic emails to cheer me up when work didn't go the way it should, for helping to send off all my stuff back to the U.K., for picking me up from the airport and much more. Martin is just class. It is the humour he provides willingly as well as the one he provides unwillingly that make your life richer. You are lucky if you have Martin around you (and I am not just talking about the countless things that somehow only happen to him...); he is a very kind person, which is overlooked by some.

And of course I thank Peter Kasper for his ornithological insights. Some of the best Sundays have been spent walking around at 7 or 8 in the morning to appreciate Fermilab's fantastic world of birds (and Peter was quite forgiving when I got my warblers, sparrows and wrens mixed up a wee bit).

Back in Glasgow I thank John (3), Meg, Fred, Val, Chris Parkes, Keith, Gavin, Julien, Marilyne, Double, Andy, Liam, Vic, Christian, Francesco, Craig, Steven (2) and some aforementioned for making meetings at $11 \mathrm{am}, 4 \mathrm{pm}$ (coffee) and Friday at $5 \mathrm{pm}$ (pub) worthwhile. Christian was a great office mate to have (although 
sometimes he took me by surprise when he answered one of my questions with a time delay of 20 minutes).

Julien and Marilyne are lost to South Africa, but I hope John and me can see them and the new addition to the Marchal family once the thesis is finally finished and we have time for a honeymoon.

Francesco is simply magic - although that word should really be reserved for Andy - and was a great enrichment to the department. It was a great pity that Keith moved further away from Glasgow so that he had to miss many of the Friday evening meetings, although he is very serious about the morning ones. Thanks to Keith also for always listening when things were going rather wrong.

Special thanks go to Liam - he was very important in keeping my sanity when things didn't go as planned. He has a talent to say what other people think, or what other people wish they would have the guts to say! And I greatly profited from this. It cheered me up, it kept me sane.

Now Glasgow would not have been the brilliant time it was, besides all the struggles of the thesis, if not for Andy and Double. Double shared a great flat with John and me, ideally located just off Byres Road (so that our little family often attracted the pub-goers after a few beers for some food and some PS2). From a fellow first-year he grew to be a good friend and then grew to be family - as he refers to himself as "the child in the house". I have to say it is not the same waking up at night, walking to the bathroom past the living room and not finding Double in the living room playing his play station.

And Andy, well, what would Glasgow have been without Andy. I do not want to know. Strange to think I only really met him after I got back from Fermilab. The time in Glasgow seems unthinkable without Andy and to me it seems he has always been there. I wouldn't have seen half as much of Glasgow without him. Thanks Andy for magic times! I still wish we could get Clare and you jobs in Munich!

Speaking of Munich I need to thank Dorothee Schaile (she knows what for) the last year would have been so much more difficult and inconvenient without her kindness. The whole ETP group deserves thanks for making my writing-up time easier and more fun. In particular I need to thank Meta who I shared an office with, Johannes who endures us even though we support FC Bayern and Tim 
\& Johanna who transformed the Friday pub meetings to Friday StuSta-Schnitzel meetings. Thanks also to Knud the new Bavarian and one of the few people that moved to where we live instead of away like Tim and now even Johanna.

Special thanks go to Otmar Biebel who was an enormous help when it came to evaluating some more theory-based systematics. As busy as he may have been his door was always open for me. Thank you!

And of course I thank Theresa, alias Resi, or Hexokinase for her friendship that dates back to school times. It outlasted time and distance and finally, now that I am back in Munich we can see more of each other again.

Last but not least I get to the people closest to me eventhough - apart from John - they have lived furthest away from me during most of the time of my thesis. Thanks to my parents and John's parents for always believing that I will finish this thesis. Thanks to John's parents also for considering me a worthy daughter-in-law before even John realised. Thanks to Marissa, the bestest sister one can have, and her husband, my friend and former flat-mate Henrik, for their letters, phone calls, for cheering me up. Well, spending a year in Australia and telling me they just saw whales when I struggled with my theory chapter was a bit cheeky, but they are forgiven (this way at least John and me got to spend an amazing holiday with them).

Thanks to my parents for their unfailing love, their survival parcels (which Double also came to enjoy), their little postcards and the smiley faces dad drew onto them and for thinking that at nearly $30 \mathrm{I}$ am still not too old for an advent calender. In the end of the day, they made all this possible and they supported me during this time every way they could. Eventhough they don't really get any day off in their bookshop they managed to come to Glasgow for a long weekend. No words can express just how grateful I am to my parents for everything they gave me.

The last big thanks go to John Alan Kennedy, my friend and my loving husband. I guess John might have had the hardest time during my Ph.D. as it is always the people closest to you that suffer most from your bad moods, from seeing you unhappy and from seeing you stressed without being able to change the situation.

Thanks for being there for me all this time, thanks for exciting times and nice and quiet times, for visiting me at Fermilab when you were so stressed yourself with 
your own Ph.D., for coming to visit Munich and for choosing it as the place for your current job. My good friends know that I really enjoyed my time in Glasgow and that I didn't quite feel ready to leave, but that my husband likes the place I was born in means a lot to me. People tend to want to go back to where they were born once they grow older. Well, we are back for now, not quite so old yet, but who knows, we might stay (and bring all our friends).

Thank you John for being proud of me (god knows why) and above all, for having had the courage to ask me to marry you. 


\section{Declaration}

Except where explicit reference is made to the work of others, this dissertation is the result of my own work. None of this material has been submitted for any other degree at the University of Glasgow or any other institution.

Tatjana Alberta Hanna Unverhau 


\section{Contents}

Contents $\quad x i$

List of Figures $\quad$ xiv

List of Tables $\quad$ xix

1 Introduction 1

2 Theoretical Overview $\quad 4$

2.1 Fundamental Constituents of Matter . . . . . . . . . . . . . 4

2.2 Interactions in the Standard Model . . . . . . . . . . . . . . 6

2.2.1 Gauge Theories and the Electromagnetic Force . . . . . . 7

2.2.2 The Strong Interaction . . . . . . . . . . . . . 11

2.2.3 The Weak Interaction . . . . . . . . . . . . 13

2.2.4 Spontaneous Symmetry Breaking and the Higgs Mechanism . . 16

2.3 Symmetries in the Standard Model . . . . . . . . . . . 18

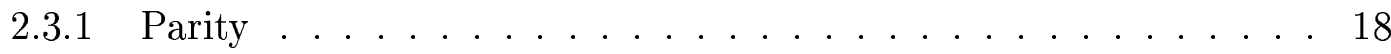

2.3.2 Charge Conjugation . . . . . . . . . . . 19

2.3.3 Time Reversal and the CPT Theorem .......... 20

2.4 B Hadron Production . . . . . . . . . . . . . . . . . 20

2.4.1 Leading Order Processes for Heavy Quark Production . . . . . . . 21

2.4.2 Next to Leading Order Processes . . . . . . . . . . . . 22

2.4 .3 Quark Fragmentation . . . . . . . . . . . 23

2.5 Decay of Heavy Hadrons . . . . . . . . . . . . . . 26

2.5.1 The Spectator Model . . . . . . . . . . . 27 
2.5.2 Diagrams with Spectator Quark Involvement and Pauli Interference 29

2.5.3 Heavy Quark Theory . . . . . . . . . . . . . . . . 31

2.5.4 Motivation for the Measurement of the $\Lambda_{b}$ Lifetime $\ldots \ldots . .32$

3 The Experimental Apparatus $\quad 34$

3.1 The Accelerator Complex . . . . . . . . . . . . . . . . . . . . 34

3.2 The Collider Detector at Fermilab . . . . . . . . . . . . . . 40

3.2 .1 Tracking Detectors . . . . . . . . . . . . . . . 43

3.2 .2 The Time-Of-Flight Detector $\ldots \ldots \ldots \ldots \ldots$

3.2 .3 The Calorimeter System . . . . . . . . . . . . . . . 51

3.2 .4 CDF's Muon System . . . . . . . . . . . . . . . 56

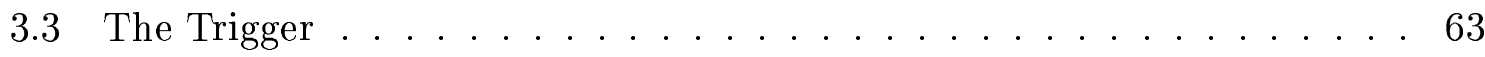

4 Event Reconstruction and Selection $\quad 69$

4.1 Track and Vertex Reconstruction . . . . . . . . . . . . . . 70

4.2 Particle Identification . . . . . . . . . . . . . . . . . 74

4.2 .1 Muon Identification . . . . . . . . . . . . . . . . . . 74

$4.2 .2 \quad$ Particle Identification via $\mathrm{dE} / \mathrm{dx} \ldots \ldots \ldots \ldots . \ldots . \ldots 75$

4.3 Offline Confirmation of the Trigger Cuts $\ldots \ldots \ldots \ldots . \ldots 77$

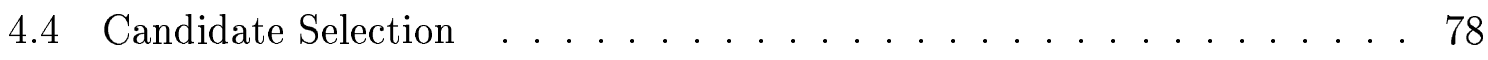

4.4 .1 Preselection . . . . . . . . . . . . . . . 78

4.4 .2 Monte Carlo Simulation . . . . . . . . . . . . . . . 81

4.4 .3 Optimisation of Selection Cuts $\ldots \ldots \ldots \ldots$

4.5 Background Processes . . . . . . . . . . . . . . . . . 102

4.5.1 Combinatorial Background and $\Lambda_{c}$ Reflections $\ldots \ldots \ldots 102$

4.5.2 Backgrounds from Real Charm Decays . . . . . . . . . . . 104

4.5.3 Other Sources of $\Lambda_{c}$ Baryons . . . . . . . . . . . . . 110

4.5.4 Background from $b \bar{b}$ and $c \bar{c} \ldots \ldots \ldots \ldots 112$

4.6 Summary . . . . . . . . . . . . . . . . . . . . . . . 113 
5 Measurement of the $\Lambda_{b}$ Lifetime $\quad 115$

5.1 Determination of the Proper Decay Length . . . . . . . . . . . . 115

5.1 .1 The K-Factor . . . . . . . . . . . . . . . . 116

5.1 .2 The Trigger Bias . . . . . . . . . . . . . . . . 117

5.2 The Lifetime Fit . . . . . . . . . . . . . . . . . . . . 123

5.2.1 The Unbinned Maximum Log Likelihood Fit . . . . . . . . . 123

5.2 .2 The Fit Function . . . . . . . . . . . . . . . 126

5.3 Validation of the Fit . . . . . . . . . . . . . . . . 129

5.3 .1 Toy Monte Carlo Studies . . . . . . . . . . . . . . . . 129

5.3 .2 Fit to the Realistic Simulation . . . . . . . . . . . . 131

6 Systematic Effects 133

7 Results and Conclusions $\quad 148$

7.1 Fixed Input Values and Distributions . . . . . . . . . . . . . . 148

7.2 Free Parameters of the Lifetime Fit . . . . . . . . . . . . . . 151

7.3 The Lifetime Fit . . . . . . . . . . . . . . . . . 152

7.4 Conclusions . . . . . . . . . . . . . . . . . . . 154

$\begin{array}{ll}\text { A Data - Monte Carlo Comparison } & 157\end{array}$

B Integration of the Fit Functions $\quad 170$

B.1 Calculation of the Signal Function . . . . . . . . . . . . 170

B.2 Calculation of the Background Function $\ldots \ldots \ldots \ldots \ldots$

$\begin{array}{ll}\text { Bibliography } & 175\end{array}$ 


\section{List of Figures}

2.1 (a) Primitive QED vertex; (b) Feynman diagram for Compton scattering. 10

2.2 Primitive QCD vertices. . . . . . . . . . . . . 12

2.3 Primitive vertices of the weak interaction. . . . . . . . . . 15

2.4 Higgs potential. . . . . . . . . . . . . . . 17

2.5 Leading order Feynman diagrams for heavy quark production. . . . . . . 21

2.6 Total production cross section for heavy quark pairs in $p \bar{p}$ collisions. . . . 22

2.7 Example Feynman diagrams for NLO contributions to flavour creation. . 23

2.8 Feynman graphs for gluon splitting and flavour excitation. . . . . . . . 24

2.9 Peterson fragmentation function. . . . . . . . . . . 26

2.10 Spectator diagram for b-mesons (a) and b-hadrons (b). . . . . . . . . 27

2.11 Lifetimes of charm and bottom hadrons. . . . . . . . . . . 28

2.12 Diagrams for Weak Annihilation (a) and W-Scattering (b) . . . . . . . 29

2.13 Helicity assignments for the decay of a $D^{0}$ meson in the $D^{0}$ restframe. . 30

2.14 External and internal spectator diagrams for $B^{-}$and $B^{0}$ mesons. . . . . 30

2.15 Feynman diagrams for the decays $\Lambda_{b}^{0} \rightarrow \Lambda_{c}^{+} l^{-} \bar{\nu}_{l}$ and $\Lambda_{c}^{+} \rightarrow p K^{-} \pi^{+} \ldots 33$

3.1 Aerial view of the accelerator complex at the Fermi National Accelerator Laboratory. . . . . . . . . . . . . . . . 35

3.2 Schematic view of the accelerator complex at the Fermi National Accelerator Laboratory. . . . . . . . . . . . . . . . 36

3.3 Layout of the anti-proton source. . . . . . . . . . . . 37

$3.4 \overline{\mathcal{N}}$ for various conditions at CDF. . . . . . . . . . . . . . 40

3.5 Solid cutaway view of the CDF II detector. . . . . . . . . . . 41

3.6 Elevation view of one half of the CDF II detector. . . . . . . . . . . 42 
3.7 Schematic layout of the silicon tracking system. . . . . . . . . . . . 44

3.8 (a) $\mathrm{r}-\phi$ view of L00 (innermost cylinder cross section) and the first two layers of the SVX II detector; (b) perspective view of the three barrels of the SVX II detector. . . . . . . . . . . . . . . . . . 45

3.9 (a) Perspective view of the ISL space frame and the silicon ladders; (b) design of a half ladder of the ISL detector. . . . . . . . . . . . 46

3.10 East endplate of the COT . . . . . . . . . . . . . . . 48

3.11 Three drift cells of the COT in superlayer 2, looking along the $z$ direction. 49

3.12 (a) Location of the TOF detector and the new calorimeter system in the plug region within the CDF II detector; (b) separation power obtained from the time difference as a function of momentum between $\mathrm{K} / \pi, \mathrm{p} / \mathrm{K}$ and $\mathrm{p} / \pi$ assuming a timing resolution of $100 \mathrm{ps} \ldots \ldots \ldots \ldots$

3.13 Development of an electromagnetic shower and a hadronic shower. . . . . 52

3.14 One wedge of the CEM with its light collecting system. . . . . . . . . 54

$3.15 \mathrm{R}-z$ view of the top half of one plug of the new calorimeter for CDF II. . 56

3.16 Location of the muon detectors for Run II in azimuth $\phi$ and pseudorapidity $\eta \ldots \ldots \ldots \ldots \ldots \ldots \ldots \ldots \ldots$

3.17 (a) Location of the central muon detector in $\mathrm{r}-\phi$ and $\mathrm{r}-z$; (b) cross section of a CMU module showing its 12 drift tube cells. . . . . . . . . . 58

3.18 Configuration of the CMP and its scintillators. . . . . . . . . 59

3.19 (a) Schematic r- $z$ view of a CMX wedge. (b) Drift cell layout for the CMX. 60

3.20 The intermediate muon system. . . . . . . . . . . . . . 62

3.21 Architecture of the three-level pipelined and buffered trigger system for CDF II. . . . . . . . . . . . . . . . . . . . . 64 64

3.22 Block diagram of the trigger system for CDF II. . . . . . . . . . . 65

3.23 Definition of the impact parameter $\mathrm{d}_{0}$ at CDF. . . . . . . . 66

4.1 Event topology for $\Lambda_{b}^{0} \rightarrow \Lambda_{c}^{+} l^{-} \bar{\nu}_{l}$ with $\Lambda_{c}^{+} \rightarrow p K^{-} \pi^{+} . \ldots \ldots 69$

4.2 Impact parameter of a track from a three prong decay for two different lifetimes of the decaying particle. . . . . . . . . . 70

4.3 Track segments and angle $\alpha$ used in segment linking. . . . . . . . . . 71 
4.4 Stopping power for positive muons in copper vs $\beta \gamma$ and energy deposited vs momentum in the TPC at PEP. . . . . . . . . . . . 76

4.5 $p K \pi$ invariant mass distribution for all three track combinations after application of a cut on the fit probability; histogram of the vertex fit

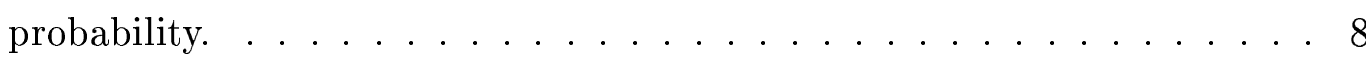

4.6 Input $\mathrm{b}$ quark $\frac{\mathrm{d} \sigma}{\mathrm{dp} \text { dy }}$ spectrum for the production of $\mathrm{B}$ hadrons with Bgenerator. . . . . . . . . . . . . . . . 81

$4.7 \quad \mathrm{p}_{\mathrm{T}} \mathrm{e}$ vs $\mathrm{p}_{\mathrm{T}} \mathrm{b}$ distribution and $\mathrm{p}_{\mathrm{T}}$ distributions for $\mathrm{b}$ quark and $\Lambda_{b}$ baryon. 82

4.8 The $p K \pi$ invariant mass distribution for events from the realistic simulation and from data. . . . . . . . . . . . . . . . 83

4.9 First set of cut variables; no selection cuts are applied. . . . . . . . . 86

4.10 Second set of cut variables; no selection cuts are applied. . . . . . . . . 87

4.11 Third set of cut variables; no selection cuts are applied. . . . . . . . . . . 88

4.12 First set of cut variables, after the application of selection cuts. . . . . . 89

4.13 Second set of cut variables, after the application of selection cuts. . . . . 90

4.14 Third set of cut variables, after the application of selection cuts. . . . . . 91

$4.15 \mathrm{c} \tau\left(\Lambda_{b}\right)$ distributions for various different cut values on $\mathrm{L}_{\mathrm{xy}}$ (p.v. $\left.-\Lambda_{\mathrm{c}}\right)$ and

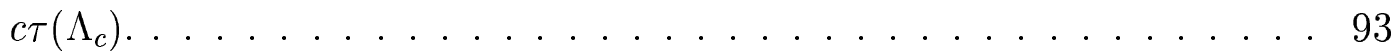

$4.16 p K \pi$ invariant mass plots before and after application of the cuts on ptratiol and ptratio $2 \ldots \ldots \ldots \ldots \ldots \ldots$

4.17 Data distribution of ptratiol and ptratio2. . . . . . . . . . 95

$4.18 \mathrm{dE} / \mathrm{dx}$ distributions obtained from data. . . . . . . . . . . . . . 97

$4.19 p K \pi$ invariant mass plots after all selection cuts, and comparing $p K \pi$ invariant mass distribution after various cuts. . . . . . . . . . . 98

4.20 First set of cut variables, after the application of all selection cuts including cuts on $\mathrm{dE} / \mathrm{dx} \ldots \ldots \ldots \ldots \ldots \ldots$

4.21 Second set of cut variables, after the application of all selection cuts including cuts on $\mathrm{dE} / \mathrm{dx} \ldots \ldots \ldots \ldots$. . . . . . . . . . . 100

4.22 Third set of cut variables, after the application of all selection cuts including cuts on $\mathrm{dE} / \mathrm{dx} \ldots \ldots \ldots \ldots$. . . . . . . . . . . . 101

$4.23 p K \pi$ invariant mass plot for data after selection cuts. . . . . . . . . 102 
$4.24 \Lambda_{c}$ reflections and the distribution $\mathrm{p}_{\mathrm{T}} \mathrm{p} / \mathrm{p}_{\mathrm{T}} \pi$ for $\Lambda_{c}$ signal MC. . . . . 103

4.25 Plots showing the background from $D^{0}$ mesons. . . . . . . . . 106

4.26 Plots showing the background from $D^{+}$mesons. . . . . . . . . . . 107

4.27 Plots showing the background from $D_{s}^{+}$mesons. . . . . . . . . . 109

4.28 Feynman diagrams for $B \rightarrow \Lambda_{c} X \ldots \ldots \ldots \ldots \ldots \ldots \ldots$

$4.29 \mathrm{~m}\left(1 \Lambda_{c}\right)$ for background from b-meson decay and signal. . . . . . . . . 111

$4.30 p K \pi$ invariant mass plot after all cuts. . . . . . . . . . . . . . . 114

5.1 Event topology for the signal $\Lambda_{b}$ decay and the decay length $L$. . . . 115

5.2 K-factor distribution before and after the application of selection cuts; mean value of the K-factor in bins of $\mathrm{p}_{\mathrm{T}}\left(1 \Lambda_{\mathrm{c}}\right) \ldots \ldots \ldots \ldots \ldots$

$5.3 c \tau\left(\Lambda_{b}\right)$ distribution before and after application of the trigger cuts; ratio of the previous $c \tau\left(\Lambda_{b}\right)$ distributions. . . . . . . . . . 118

5.4 SVT tracking efficiency curves. . . . . . . . . . . . . . . 120

5.5 Scale factor for the realistic simulation and $c \tau_{\text {reco }}^{\prime}-c \tau_{\text {gen }}^{\prime} \ldots \ldots \ldots . . .121$

$5.6 c \tau$ efficiency curve for run 142110 of the realistic simulation and for data. 122

5.7 Distribution of $c \tau$ and $c \tau^{\prime}$ and fit results of an unbinned log likelihood fit. 125

5.8 Fit of a Gaussian and a first order polynomial to the $p K \pi$ invariant mass distribution. . . . . . . . . . . . . . . . 127

5.9 Distributions showing the mean $c \tau$ values and pull distributions for toy experiments generated to test the fit. . . . . . . . . . . . 131

$5.10 \mathrm{~K}$-factor and $c \tau$ efficiency distribution used for the fit to the realistic simulation. . . . . . . . . . . . . . . . . 132

6.1 SVT tracking efficiency and $c \tau$ efficiency curves. . . . . . . . . 135

6.2 Peterson fragmentation function for four different values of $\epsilon \ldots \ldots \ldots 141$

6.3 Distribution of $\cos \theta$ for various cuts. . . . . . . . . . . 143

6.4 $p K \pi$ invariant mass plots for events containing a muon or anti-muon respectively. . . . . . . . . . . . . . . . . . 145

$7.1 \quad$ K-factor and $c \tau$ efficiency curve for data fit. . . . . . . . . . . . . 149

7.2 Fit of a Gaussian and a first order polynomial to the $p K \pi$ invariant mass distribution. . . . . . . . . . . . . . . . . 150 
7.3 Lifetime fit to the data. . . . . . . . . . . . . . . . . 153

7.4 Results of measurements of the $\Lambda_{b}$ lifetime. . . . . . . . . . 156

A.1 Comparison between signal distributions of the realistic simulation and data (sideband subtracted). . . . . . . . . . . . . . . 158

A.2 Comparison between signal distributions of the realistic simulation and data (sideband subtracted). . . . . . . . . . . . . . . 159

A.3 Comparison between signal distributions of the realistic simulation and data (sideband subtracted). . . . . . . . . . . . . . . 160

A.4 Comparison between signal distributions of the realistic simulation and data (sideband subtracted). . . . . . . . . . . . . . . . 161

A.5 Comparison between signal distributions of the parametric simulation and data (sideband subtracted) . . . . . . . . . . . . . 162

A.6 Comparison between signal distributions of the parametric simulation and data (sideband subtracted) . . . . . . . . . . . . . 163

A.7 Comparison between signal distributions of the parametric simulation and data (sideband subtracted) . . . . . . . . . . . . . . 164

A.8 Comparison between signal distributions of the parametric simulation and data (sideband subtracted) . . . . . . . . . . . . . 165

A.9 Comparison between signal distributions of the parametric simulation

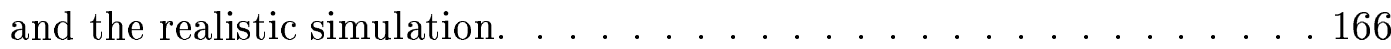

A.10 Comparison between signal distributions of the parametric simulation and the realistic simulation. . . . . . . . . . . . . 167

A.11 Comparison between signal distributions of the parametric simulation and the realistic simulation. . . . . . . . . . . . . 168

A.12 Comparison between signal distributions of the parametric simulation and the realistic simulation. . . . . . . . . . . . . . 169 


\section{List of Tables}

2.1 The three generations of fundamental fermions. . . . . . . . . 5

2.2 The three interactions described by the Standard Model together with their force mediating vector bosons. . . . . . . . . . . . 6

2.3 B quark fragmentation fractions. . . . . . . . . . . . . . 24

2.4 Lifetimes of charm and bottom hadrons. . . . . . . . . . . . . 28

2.5 Theoretical predictions and experimental results for the ratio of exclusive lifetimes of $b$ hadrons. . . . . . . . . . . . . . . 32

3.1 Position Resolution of the components of the CDF tracking system. . . . 49

5.1 Parameters of the SVT efficiency and the impact parameter resolution. . 123

5.2 Parameters from a fit to the $c \tau$ efficiency. . . . . . . . . . . . 123

5.3 Fit results from a blind test to four sets of simulated signal samples. . . . 129

5.4 Fit results from a blind test to eight sets of simulated samples containing signal events in the presence of background. . . . . . . . . . . 130

5.5 Results of the fits to the realistic simulation. . . . . . . . . 132

6.1 Measurements of the $\Lambda_{b}$ polarisation at LEP. . . . . . . . . . . . . . 142

6.2 Summary of the sources of the systematic error of the lifetime fit. . . . . 147

7.1 Fit parameters for the final lifetime fit to data. . . . . . . . . 154

7.2 Results of measurements of the $\Lambda_{b}$ lifetime from previous experiments. .155 


\section{Chapter 1}

\section{Introduction}

Inquisitiveness is a distinguishing feature of human nature. Today there are multitudinous scientific areas that explore the macroscopic and microscopic phenomena of the universe we live in, satisfying mankind's thirst for knowledge. Particle physics in particular is focused upon answering the intriguing age-old question "What is the world made of?". First accounts of this question date back approximately 2500 years to ancient Greece. The answers have changed drastically over the centuries with advances in technical equipment and theoretical knowledge. There have been ideas such as that basic matter is water, or that all matter is composed of four basic natural elements: fire, water, earth and air.

The first concept of elementary particles dates back to Democritus of Abdera, who lived from 460-371 BC, and his teacher Leucippus. He introduced the principle of non-continuous matter. Subdividing matter ever further one would eventually reach a limit and be left with an invisible and indivisible substance. After the Greek word for indivisible, atomos, he called them atoms. This idea did not gain popularity until the early $18^{\text {th }}$ century, when the Frenchman Joseph Gay-Lussac found that water is always produced with the same ratio of oxygen and hydrogen. To him this indicated that the elements consist of basic units. John Dalton took this idea a step further, proposing that every element has its own type of atoms and that all atoms of one element are identical. A classification for those elements was derived by the Russian chemist Dmitri Ivanovich Mendele'ev in 1869. He ordered them according to their atomic weight and discovered that the properties of elements seem to change 
periodically. From unfilled places in his table he predicted new elements with specific properties. Their subsequent discovery proved to be a great success for his model and up to this day people work with the periodic table of elements.

However atoms did not remain the indivisible building blocks of matter for long. The electron, a particle considered elementary to present knowledge, was discovered by J. J. Thomson in 1897 from cathode rays. Thompson correctly surmised that these charged particles are constituents of the neutral atoms. To achieve neutrality he assumed that the electrons are submerged in a positively charged entity - like plums in a pudding. Rutherford found later from his scattering experiments of alpha particles on gold foil that atoms are composed of electrons situated in a large empty space around a concentrated positive nucleus. This atomic model of Rutherford was developed further by Niels Bohr describing discrete electron orbits around the positively charged nucleus which explain distinct emission lines in atomic spectra.

With Chadwick's discovery of the neutron in 1932, the theory of the fundamental building blocks of matter seemed simpler again - different numbers of electrons, protons and neutrons could be put together to form all elements listed in the periodic table. However, even though these particles still comprised the main components of ordinary matter, more and more particles were discovered in the following decades in accelerator experiments and cosmic radiation that did not fit into this pattern. The world of elementary particles seemed complicated again up until the proposition of quarks by Gell-Mann and Zweig and the subsequent experimental discovery of evidence for their existence. The quarks were proposed as constituents of a whole family of particles, the hadrons. Protons and neutrons belong to this family as well as a particle called the $\Lambda_{b}$ baryon.

The $\Lambda_{b}$ baryon, which consists of three such quarks, is the main focus of this thesis and its experimental discovery was first announced in 1991 by an experiment conducted at CERN, the European centre for particle physics near Geneva in Switzerland. This thesis presents a measurement of the lifetime of the $\Lambda_{b}$ baryon in its semileptonic channel. Computing weak decays of heavy hadrons is a difficult task owing to the non-trivial interplay of strong and electroweak forces. However today there exist various tools that allow the theoretical prediction of lifetime ratios of bottom hadrons which can then be tested experimentally. The experimental values 
agree reasonably well with the theoretical predictions, although the lifetime of the $\Lambda_{b}$ baryon is a little shorter than expected from theory. The measurements are however still statistically limited. The Tevatron is currently the only accelerator that can produce $\Lambda_{b}$ baryons and thus provides a unique opportunity to throw further light into this area.

This thesis is organised as follows. Chapter 2 introduces in more detail our present model of sub-atomic particles, the Standard Model of fundamental particles and interactions, predictions of which have been verified with extremely high precision in modern experiments. The $\Lambda_{b}$ baryon is introduced and its lifetime measurement is motivated.

While theoretical advancements have led to new and exciting theories about the fundamental particles and interactions, the technical achievements of the last century have led to ever more sophisticated experimental techniques and advanced particle accelerators reaching ever higher energies that allow us to test those theories and explore the properties of fundamental and composite particles. Chapter 3 describes the Tevatron accelerator and the CDF detector used to provide and record the data for this thesis. The Tevatron collider currently provides the world's largest centre-of-mass energies and it is the sole accelerator capable of producing $\Lambda_{b}$ particles and thus to allow the study of their properties.

Chapter 4 describes the reconstruction and selection of the desired events, while chapter 5 introduces the lifetime measurement and fitting mechanism. Possible systematic uncertainties on the measurement are investigated and quantified in chapter 6 . Finally chapter 7 details and discusses the result of the $\Lambda_{b}$ lifetime measurement. 


\section{Chapter 2}

\section{Theoretical Overview}

The Standard Model (SM) of particle physics quantitatively describes all interactions of the fundamental particles, with the exclusion of gravity. The interactions within this model are formulated in terms of Quantum Field Theory (QFT), as gauge theories. As yet there exists no satisfactory description of gravity at the quantum level. However the effects of gravity are negligible in high energy physics processes and thus a discussion of gravity is omitted from this thesis.

The following section presents an introduction to the particle content of the Standard Model, a brief discussion of the underlying principle of gauge theories and an overview of the physics relevant to the decays of $b$ hadrons.

\subsection{Fundamental Constituents of Matter}

Particles can be classified according to their spin statistics as either bosons, particles with integer spin (in units of $\hbar$ ), or fermions, particles with half integer spin. Quarks and leptons, carrying spin $1 / 2$, belong to the latter group and are thought to be the fundamental constituents of matter. Today we know of 12 different types of fundamental fermions, six leptons and six quarks. For each of these particles there exists an antiparticle with the same mass but opposite electric charge.

Probably the best known lepton is the electron $\left(e^{-}\right)$, which was the first fundamental particle to be discovered. The muon $\left(\mu^{-}\right)$and the tau particle $\left(\tau^{-}\right)$behave much like the electron, only their mass is larger. Each of these leptons has an associated low mass neutrino with zero electric charge. While all leptons are subject to the 


\begin{tabular}{|c|c|c|c|c|}
\hline \multirow[t]{4}{*}{ Quarks } & Generation & Symbol & $\begin{array}{l}\text { Electric } \\
\text { Charge }\end{array}$ & Mass $\left(\mathrm{GeV} / \mathrm{c}^{2}\right)[1]$ \\
\hline & $\bar{~} 1$. & $\begin{array}{l}\mathrm{u} \\
\mathrm{d}\end{array}$ & $\begin{array}{l}+2 / 3 \\
-1 / 3 \\
\end{array}$ & $\begin{array}{c}0.0015 \text { to } 0.0045 \\
0.005 \text { to } 0.0085\end{array}$ \\
\hline & 2. & $\begin{array}{l}\mathrm{c} \\
\mathrm{s}\end{array}$ & $\begin{array}{l}+2 / 3 \\
-1 / 3\end{array}$ & $\begin{array}{c}1.0 \text { to } 1.4 \\
0.080 \text { to } 0.155\end{array}$ \\
\hline & 3. & $\begin{array}{l}\mathrm{t} \\
\mathrm{b}\end{array}$ & $\begin{array}{l}+2 / 3 \\
-1 / 3\end{array}$ & $\begin{array}{c}174.3 \pm 5.1 \\
4.0 \text { to } 4.5\end{array}$ \\
\hline
\end{tabular}

\section{Leptons}

\begin{tabular}{|l|c|c|c|}
\hline Generation & Symbol & $\begin{array}{c}\text { Electric } \\
\text { Charge }\end{array}$ & Mass $\left(\mathrm{MeV} / \mathrm{c}^{2}\right)[1]$ \\
\hline \hline 1. & $\nu_{\mathrm{e}}$ & 0 & $<0.000003$ \\
& $\mathrm{e}^{-}$ & -1 & $0.510998902 \pm 0.000000021$ \\
\hline 2. & $\nu_{\mu}$ & 0 & $<0.19$ \\
& $\mu^{-}$ & -1 & $105.658357 \pm 0.000005$ \\
\hline 3. & $\nu_{\tau}$ & 0 & $<18.2$ \\
& $\tau^{-}$ & -1 & $1776.99_{-0.26}^{+0.29}$ \\
\hline
\end{tabular}

Table 2.1: The three generations of fundamental fermions. The large uncertainties in the quark masses arise since quarks do not exist as free particles. The large top mass however permits direct measurements, as the top quark decays before hadronisation.

weak interaction, only the charged leptons interact electromagnetically. Quarks, on the other hand, carry an additional quantum number called colour that subjects them to the strong force thus making them participants of all interactions. The six quarks are, in order of increasing mass, the up (u), down (d), strange (s), charm (c), bottom (b) and top (t) quark. Each quark can occur in the three different colour states, conventionally referred to as red, green and blue.

These fundamental quarks and leptons can be grouped into three families or generations, according to increasing mass. The generations reflect each other in their behaviour under the strong, electromagnetic and weak force. The 12 fermions, their masses and their electric charge are shown in table 2.1, where they are arranged into the three generations.

At present it is unclear whether there could be a fourth generation of fermions. Any additional generation would have to contain much heavier particles to agree with the present experimental data, since precise measurements of the $\mathrm{Z}$ Boson width at LEP have determined the number of generations of light neutrinos $\left(m_{\nu}<m_{\mathrm{Z}} / 2\right)$ to be three. Thus far there also exists no evidence to support theories which suggest 
that fermions are composite particles, even though the grouping into families could hint towards a substructure.

\subsection{Interactions in the Standard Model}

In addition to the fundamental fermions the Standard Model includes a description of the force carrying particles, the gauge bosons, and the force or interaction they mediate between fermions and themselves. Four fundamental forces are known today. They are, in order of decreasing strength, the strong force, the electromagnetic force, the weak force and the gravitational force. While the last of these is not included in the Standard Model, the electromagnetic and weak force find a unified description reducing the four interactions to three. Table 2.2 lists the interactions treated in the Standard Model together with their respective force mediating particles.

\begin{tabular}{|c|lr|c|c|}
\hline Force & \multicolumn{2}{|c|}{ Mediator } & Electric Charge & Mass $\left(\mathbf{G e V} / \mathbf{c}^{\mathbf{2}}\right)$ \\
\hline strong & gluon & $\mathrm{g}$ & 0 & 0 \\
\hline electromagnetic & photon & $\gamma$ & 0 & 0 \\
\hline weak & & & & \\
charged & W boson & $W^{ \pm}$ & \pm 1 & $80.423 \pm 0.039[1]$ \\
neutral & Z boson & $Z$ & 0 & $91.1876 \pm 0.0021[1]$ \\
\hline
\end{tabular}

Table 2.2: The three interactions described by the Standard Model together with their force mediating vector bosons.

As mentioned above, all interactions in the Standard Model are described in terms of QFT. The interactions are derived through the requirement that the invariance of the Lagrangian of a system under global gauge transformations should hold locally, i.e., under space-time dependent transformations.

The idea of local gauge invariance was first introduced by the work of Hermann Weyl in 1919 [2]. Applying the demand for local gauge invariance to the Dirac Lagrangian for a free electron generates the complete theory of electrodynamics. The concept of gauge theories is summarised here following the case of electrodynamics. Although this presents the simplest case it contains all the important concepts. Quantum ElectroDynamics (QED) is the oldest, simplest and most successfully tested of the dynamical theories in the Standard Model. 


\subsubsection{Gauge Theories and the Electromagnetic Force}

Starting from the theory of a free electron, described by its four-component complex spinor field $\Psi_{i}(\mathrm{i}=0,1,2,3)$, the Lagrangian density for a free Dirac field $\Psi$ is

$$
\mathcal{L}=\bar{\Psi}(x)\left(i \gamma^{\mu} \partial_{\mu}-m\right) \Psi(x)
$$

$m$ is the electron mass, $\gamma^{\mu}$ are the $4 \times 4$ Dirac matrices and the adjoint spinor $\bar{\Psi}$ represents $\Psi^{\dagger} \gamma^{0}$ with ${ }^{\dagger}$ denoting the transpose conjugate. One explicit (but not unique) representation of the Dirac matrices is:

$$
\begin{aligned}
\gamma^{0} & =\left(\begin{array}{cccc}
1 & 0 & 0 & 0 \\
0 & 1 & 0 & 0 \\
0 & 0 & -1 & 0 \\
0 & 0 & 0 & -1
\end{array}\right), \gamma^{1}=\left(\begin{array}{cccc}
0 & 0 & 0 & 1 \\
0 & 0 & 1 & 0 \\
0 & -1 & 0 & 0 \\
-1 & 0 & 0 & 0
\end{array}\right), \\
\gamma^{2} & =\left(\begin{array}{cccc}
0 & 0 & 0 & -i \\
0 & 0 & i & 0 \\
0 & i & 0 & 0 \\
-i & 0 & 0 & 0
\end{array}\right), \gamma^{3}=\left(\begin{array}{cccc}
0 & 0 & 1 & 0 \\
0 & 0 & 0 & -1 \\
-1 & 0 & 0 & 0 \\
0 & 1 & 0 & 0
\end{array}\right) .
\end{aligned}
$$

Phase transformations of the kind $\Psi(x) \rightarrow \Psi^{\prime}(x)=e^{i \Theta} \Psi(x)^{1}$ are called global if the real phase $\Theta$ is the same for all space-time points $x$. The Lagrangian of equation 2.1 is invariant under such a transformation; $\bar{\Psi}$ transforms to $\bar{\Psi} e^{-i \Theta}$, and in the $\bar{\Psi} \Psi$ combination terms the exponential factors cancel. If on the other hand we apply a local gauge transformation, i.e., we allow the phase to vary from point to point, $\Psi(x) \rightarrow \Psi^{\prime}(x)=e^{i \Theta(x)} \Psi(x)$, the free Dirac Lagrangian is no longer invariant since we pick up an extra term from the derivative of $\Theta(=\Theta(x))$ :

$$
\partial_{\mu}\left(e^{i \Theta} \Psi\right)=i\left(\partial_{\mu} \Theta\right) e^{i \Theta} \Psi+e^{i \Theta} \partial_{\mu} \Psi
$$

resulting in

$$
\mathcal{L} \rightarrow \mathcal{L}-\left(\partial_{\mu} \Theta\right) \bar{\Psi} \gamma^{\mu} \Psi
$$

For the following it is helpful and more intuitive to replace $\Theta$ with $q \lambda(x)$, identifying $q$ as charge. To obtain a locally invariant Lagrangian we have to add an extra

\footnotetext{
${ }^{1}$ The set of all such phase transformations is the group $\mathrm{U}(1)$. As $e^{i \Theta_{1}} e^{i \Theta_{2}}=e^{i \Theta_{2}} e^{i \Theta_{1}}$, it is an Abelian group.
} 
term to the free Lagrangian to cancel the additional derivative $\partial_{\mu} \Theta \bar{\Psi} \gamma^{\mu} \Psi$. Suppose we add a term $-q \bar{\Psi} \gamma^{\mu} \Psi A_{\mu}$, resulting in the Lagrangian

$$
\mathcal{L}=\bar{\Psi}(x)\left(i \gamma^{\mu} \partial_{\mu}-m\right) \Psi(x)-q \bar{\Psi} \gamma^{\mu} \Psi A_{\mu}
$$

where $A_{\mu}$ is a new vector field, called a gauge field. Requiring the field to transform as

$$
A_{\mu} \rightarrow A_{\mu}^{\prime}=A_{\mu}-\partial_{\mu} \lambda
$$

renders the Lagrangian invariant under the local gauge transformation defined above. However, if this field is to be identified with a physical force mediating particle, in this case the photon, the Lagrangian for the added field $A_{\mu}$ needs a kinetic term. The full Lagrangian for a free vector field is

$$
\mathcal{L}=-\frac{1}{16 \pi} F^{\mu \nu} F_{\mu \nu}+\frac{1}{8} \pi m_{A} A^{\nu} A_{\nu} .
$$

While $F^{\mu \nu}=\partial^{\mu} A^{\nu}-\partial^{\nu} A^{\mu}$ remains invariant if $A_{\mu}$ is transformed according to equation 2.4, the mass term does not. Therefore, if the latter term is not to break the invariance, the field $A_{\mu}$ has to be massless. Thus, by demanding local invariance, we arrive at

$$
\mathcal{L}=\bar{\Psi}(x)\left(i \gamma^{\mu} \partial_{\mu}-m\right) \Psi(x)-q \bar{\Psi} \gamma^{\mu} \Psi A_{\mu}+\frac{-1}{16 \pi} F^{\mu \nu} F_{\mu \nu},
$$

which is the Lagrangian for Quantum Electro Dynamics (QED). The first term describes the kinetic energy and mass of $\Psi$, the second determines the interaction - how the field $A_{\mu}$ couples to $\Psi$ via the charge q - and the third term contains the kinetic energy of $A_{\mu}$, which is precisely the electromagnetic potential. The last two terms reproduce the Maxwell Lagrangian for a massless vector field with the current density $J^{\mu}=q\left(\bar{\Psi} \gamma^{\mu} \Psi\right)$. Demanding that global gauge invariance should hold locally has lead to the generation of electrodynamics. The mediating particle can be identified as the photon, which is indeed massless.

As a recipe for obtaining a locally gauge invariant Lagrangian for an interaction, the form of the Lagrangian in equation 2.3 can also be derived by introducing the covariant derivative $D_{\mu} \equiv \partial_{\mu}+i q A_{\mu}$. Then by replacing $\partial_{\mu}$ with $D_{\mu}$ in the free Lagrangian (equation 2.1) and letting $A_{\mu}$ transform as $A_{\mu}^{\prime} \rightarrow A_{\mu}-\partial_{\mu} \lambda$ the Lagrangian 
is invariant under $\Psi \rightarrow \Psi e^{i q \lambda(x)}$. Then it only remains for the kinetic terms of the new (massless) field to be added.

The gauge transformation can also be described as a multiplication of the wave function by a term $e^{i H} ; H$ is, in the case of electrodynamics, simply a phase factor and as such the Lagrangian is said to be symmetric under an Abelian gauge transformation. In 1954 Yang and Mills extended the idea from the Abelian case of electrodynamics (with the photon representing the introduced gauge field) to the non-Abelian case of the group $\mathrm{SU}(2)$. Here, $H$ is a $2 \times 2$ hermitian matrix and in this case three massless vector fields are required to provide local gauge invariance; yet at that time no isotriplet of particles with the necessary features was known. However, Yang and Mills had shown that it is in principle possible to extend Weyl's idea to the non-Abelian case. The power and generality of local gauge invariance was not fully appreciated until the early 70's when Gerard 't Hooft showed [3,4] that gauge theories are automatically renormalisable.

Recapitulating, the photon is the mediator of the electromagnetic force, which acts on all charged particles. As the photon itself is neutral there is no direct interaction between photons themselves. The elementary process for electromagnetic interactions is shown in figure 2.1 (a). The denotation "e" stands for any charged particle and " $\gamma$ " denotes the photon as customary. Processes such as $e^{-} \rightarrow e^{-}+\gamma$ and $e^{-}+e^{+} \rightarrow \gamma$ on their own, i.e. processes consisting of only the primitive vertex depicted in figure 2.1 (a) do not occur in nature. They are forbidden by energy and momentum conservation respectively. However combining two or more such vertices will lead to all possible processes described by QED. Such combined diagrams representing physical processes are examples of Feynman diagrams. Figure 2.1 (b) shows the lowest order Feynman diagram for Compton scattering. Time increases here from left to right with particles indicated as travelling forwards in time and antiparticles indicated as travelling backwards.

Although these diagrams are only graphical representations and do not indicate the actual momentum direction of each particle participating in the interaction, they are very useful tools for calculating cross sections and decay rates. For each diagram there exist rules which allow the calculation of a number which ultimately 


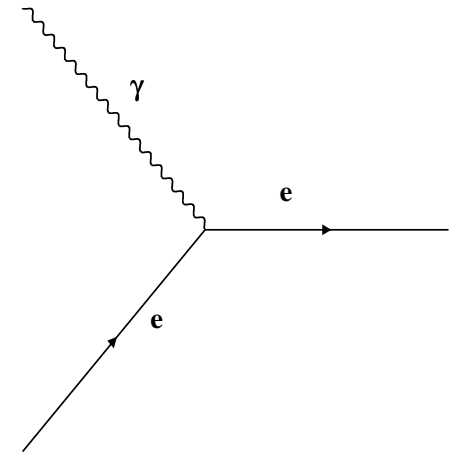

(a)

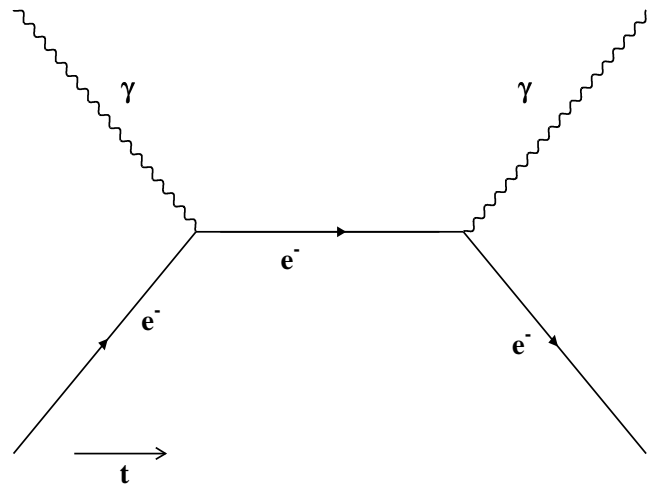

(b)

Figure 2.1: Figure (a) shows the primitive QED vertex; e represents any charged particle and $\gamma$ stands for the photon. Figure (b) shows the lowest order diagram for Compton scattering.

corresponds to the transition amplitude $M$ for the process in question. These amplitudes occur in formulae both for cross sections and decay rates. Energy and momentum are conserved at each vertex and the mass of particles on internal lines is determined by this conservation of energy and momentum rather than by the nature of the particle. To calculate the amplitude of a certain process all diagrams with the same external lines, i.e. all diagrams with the same initial and final state have to be added. Each vertex of a particular interaction contributes to such calculations proportional to the respective coupling constant. In the case of QED the strength is proportional to the coupling constant $g$ which is related to the fine structure constant $\alpha$ via $g=\sqrt{4 \pi \alpha}$. Even though $g$ is called a coupling constant, the strength of electromagnetic interactions varies with momentum transfer, increasing slightly with increasing energy. While the value of $\alpha$ is $1 / 137$ for low energies it grows to $1 / 128$ at the mass of the $\mathrm{W}$ boson. The small size of this coupling constant allows QED processes to be approximated by a perturbation series. Each term in this series can be expressed with a Feynman diagram and calculated according to the Feynman rules. Terms of higher order in $\alpha$, i.e. terms with more vertices and internal loops, contribute less to the final result and a calculation of the first terms in the perturbation series leads to good approximations of QED processes. 


\subsubsection{The Strong Interaction}

The first application of the gauge principle in the non-Abelian case was the description of the strong interaction. Analogously to the electric charge, particles that are subject to this force (the quarks) carry a charge referred to as colour (therefore "chromo" dynamics). They exist in three colours, referred to as red, green, and blue. The gauge theory describing the strong interaction is known as Quantum Chromo Dynamics (QCD). It is based on the non-Abelian group $\mathrm{SU}(3)_{\mathrm{c}}$, where "c" stands for colour and the three dimensions originate from the three possible colour states of quarks. Demanding the Lagrangian of the system to be invariant under local gauge transformations leads to the introduction of eight massless gauge fields, the quanta of QCD, which are in nature realised by eight massless gluons. The covariant derivative, acting on the three quark spinor,

$$
q=\left(\begin{array}{l}
q_{1} \\
q_{2} \\
q_{3}
\end{array}\right)
$$

in the case of QCD is

$$
D_{\mu}=\partial^{\mu}+i g_{s}\left(\frac{\lambda_{\alpha}}{2}\right) A_{\mu}^{\alpha}
$$

where $A_{\mu}^{\alpha}$ are the eight gluon fields and $\frac{\lambda_{\alpha}}{2}$ are the generators of the group $\mathrm{SU}(3)$. The Gell-Mann matrices $\lambda_{\alpha}$ are:

$$
\begin{gathered}
\lambda_{1}=\left(\begin{array}{lll}
0 & 1 & 0 \\
1 & 0 & 0 \\
0 & 0 & 0
\end{array}\right), \lambda_{2}=\left(\begin{array}{ccc}
0 & -i & 0 \\
i & 0 & 0 \\
0 & 0 & 0
\end{array}\right), \lambda_{3}=\left(\begin{array}{ccc}
1 & 0 & 0 \\
0 & -1 & 0 \\
0 & 0 & 0
\end{array}\right) \\
\lambda_{4}=\left(\begin{array}{lll}
0 & 0 & 1 \\
0 & 0 & 0 \\
1 & 0 & 0
\end{array}\right), \lambda_{5}=\left(\begin{array}{ccc}
0 & 0 & -i \\
0 & 0 & 0 \\
i & 0 & 0
\end{array}\right) \\
\lambda_{6}=\left(\begin{array}{lll}
0 & 0 & 0 \\
0 & 0 & 1 \\
0 & 1 & 0
\end{array}\right), \lambda_{7}=\left(\begin{array}{ccc}
0 & 0 & 0 \\
0 & 0 & -i \\
0 & i & 0
\end{array}\right), \lambda_{8}=\frac{1}{\sqrt{3}}\left(\begin{array}{ccc}
1 & 0 & 0 \\
0 & 1 & 0 \\
0 & 0 & -2
\end{array}\right) .
\end{gathered}
$$

Gluons, the mediators of the strong force, carry a combination of colour and anticolour. This results in additional primitive vertices that account for the self coupling of gluons. Colour is conserved at each vertex of QCD. The three primitive vertices of QCD are depicted in figure 2.2. Up to this day no free quarks have been observed; they are confined within experimentally observable colourless particles called 

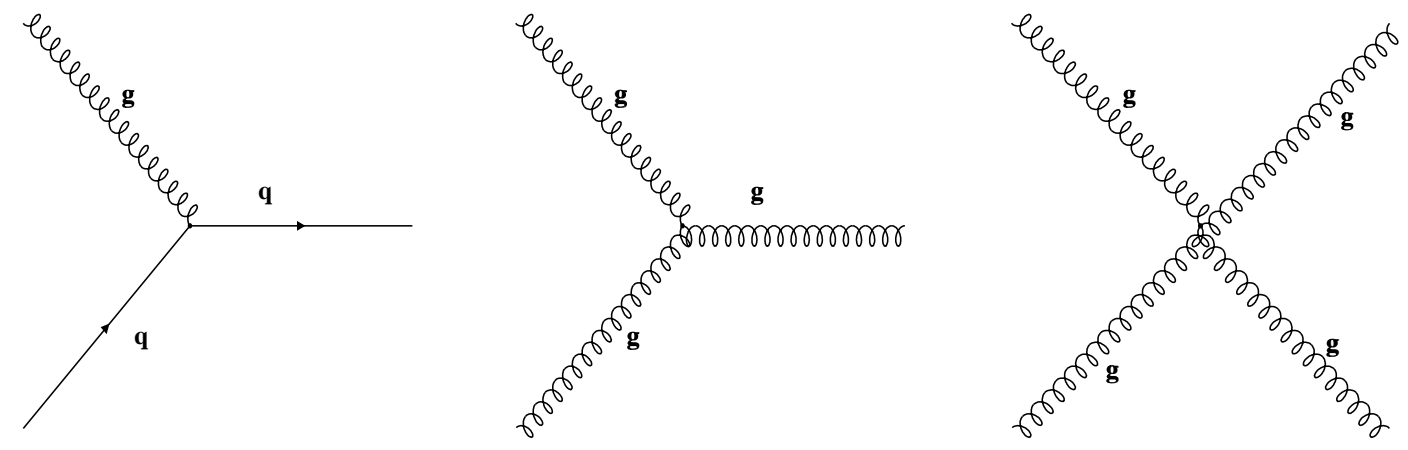

Figure 2.2: Primitive QCD vertices. The first vertex represents the coupling of gluons to quarks. Colour is conserved at each vertex. Looking at the leftmost diagram, if the incoming quark is red and the outgoing quark is blue, then the incoming gluon has to carry one unit of anti-red and one unit of blue. The self coupling of gluons results in the three and four quark vertices.

hadrons. Today we know of two types of hadrons, baryons and mesons. Baryons are fermions consisting of three quarks, such as the proton and the neutron which have a quark content $u u d$ and $u d d$ respectively. The three quarks carry together one unit of red, one unit of green and one unit of blue resulting in a colourless, or white state. Mesons such as the $\pi^{+}(u \bar{d})$ are bosons consisting of a quark and an antiquark. To result in a colourless state the quark and the antiquark inside a meson have to carry colour and anticolour, e.g. if the quark is red, the antiquark has to be anti-red. In theory more complicated composite particles could exist as long as they are colourless.

The coupling of the strong interaction $g_{s}=\sqrt{4 \pi \alpha_{s}}$ takes, as the name indicates, much larger values than the coupling of the electromagnetic interaction. The coupling strength is again dependent on the distance between particles and on the momentum transfer, $\mathrm{Q}$, between them. In the case of $\mathrm{QCD}$, the coupling decreases with increasing momentum transfer Q. This is the typical behaviour for non-Abelian gauge theories where the field particles carry charge and are self-coupling. The coupling constant can be expressed in terms of a single parameter $\Lambda$, the scale of QCD. In lowest order this relation is:

$$
\alpha_{s}\left(Q^{2}\right)=\frac{12 \pi}{(11 n-2 f) \ln \left(\frac{q^{2}}{\Lambda^{2}}\right)} \text { for } q^{2} \gg \Lambda^{2},
$$


where $n$ is the number of colours and $f$ is the number of flavours. In the SM, (11n$2 f$ ) is larger than zero yielding the characteristic behaviour of a coupling constant that decreases with increasing energy. At large values of $Q^{2}$ the coupling becomes small enough for processes to be calculated perturbatively and the quarks can be regarded as quasi-free particles. This feature of QCD is referred to as asymptotic freedom. The opposite effect, that at increasing distances and thus small $Q^{2}$ the coupling becomes strong, is referred to as confinement. This effect is most likely responsible for the observation of only colour singlet states in nature, where quarks are confined into hadrons.

If quarks move apart from each other (for instance after their creation in a high energy collision), the further they move apart the larger the binding energy between them becomes, so that eventually it is energetically more favourable to create new $q \bar{q}$ pairs, resulting in more and more colour singlet objects until the energy of the quarks is sufficiently low.

\subsubsection{The Weak Interaction}

The weak interaction was first derived by Enrico Fermi as a point-like interaction with a 4-particle coupling and as such no force mediating bosons. This description is a good approximation for low energies but at higher energies certain weak processes violate unitarity. The first attempts to describe the weak interaction on its own as an $\mathrm{SU}(2)$ gauge theory failed and it became apparent that the weak interaction could only be described in the framework of gauge theories if combined with the electromagnetic interaction. Glashow, Weinberg and Salam finally succeeded in deriving both forces as different components of a single gauge theory. The underlying gauge group is the direct product of $\mathrm{SU}(2)_{\mathrm{L}}$ and $\mathrm{U}(1)_{\mathrm{Y}}$. L stands for left handed, expressing that the weak interaction is parity ${ }^{2}$ violating and thus left and right handed components of the fields should be treated separately. Y denotes the weak hypercharge, which is defined as

$$
\frac{\mathrm{Y}}{2}=Q_{e m}-I_{3}
$$

\footnotetext{
${ }^{2} \mathrm{~A}$ parity transformation is a discrete transformation that changes the sign of the space coordinates $\mathrm{x}, \mathrm{y}$ and $\mathrm{z}$ (see section 2.3).
} 
where $I_{3}$ is the third component of the weak isospin $I$ and $Q_{e m}$ is the electromagnetic charge. To ensure that the groups $\mathrm{U}(1)_{\mathrm{Y}}$ and $\mathrm{SU}(2)_{\mathrm{L}}$ commute, $\mathrm{Y}$ has to take the same value for the charged fermion and the neutrino fields. Fermion fields in this theory are separated into left and right handed components. The left-handed electron and neutrino fields form doublets, while in absence of right-handed neutrinos the right-handed electron fields form singlets of weak isospin:

$$
\begin{gathered}
f_{L}=\left(\begin{array}{c}
\nu_{e} \\
e
\end{array}\right)_{L}\left(\begin{array}{c}
\nu_{\mu} \\
\mu
\end{array}\right)_{L}\left(\begin{array}{c}
\nu_{\tau} \\
\tau
\end{array}\right)_{L}\left(\begin{array}{c}
u \\
d^{\prime}
\end{array}\right)_{L}\left(\begin{array}{c}
c \\
s^{\prime}
\end{array}\right)_{L}\left(\begin{array}{c}
t \\
b^{\prime}
\end{array}\right)_{L} \begin{array}{c}
I_{3}=1 / 2 \\
I_{3}=-1 / 2
\end{array} \\
f_{R}=e_{R}, \mu_{R}, \tau_{R}, u_{R}, c_{R}, t_{R}, d_{R}, s_{R}, b_{R} \quad I=0 .
\end{gathered}
$$

Unlike leptons, quarks of different families interact with each other via the weak interaction with interactions across generations less likely to occur than interactions within a generation. The eigenstates of the weak interaction $d^{\prime}, s^{\prime}$ and $b^{\prime}$ are related to the physical quarks or mass eigenstates $d, s$ and $b$ via the $3 \times 3$ Cabibbo-KobayashiMaskawa matrix (CKM matrix):

$$
\left(\begin{array}{l}
d^{\prime} \\
s^{\prime} \\
b^{\prime}
\end{array}\right)=\left(\begin{array}{lll}
V_{u d} & V_{u s} & V_{u b} \\
V_{c d} & V_{c s} & V_{c b} \\
V_{t d} & V_{t s} & V_{t b}
\end{array}\right)\left(\begin{array}{c}
d \\
s \\
b
\end{array}\right)
$$

If this matrix were diagonal, then "up-ness"+"down-ness" would be exactly conserved in weak processes; however the experimental values of the matrix elements [1] do show cross generation interactions:

$$
\left(\begin{array}{ccc}
0.9741 \text { to } 0.9756 & 0.219 \text { to } 0.226 & 0.0025 \text { to } 0.0048 \\
0.219 \text { to } 0.226 & 0.9732 \text { to } 0.9748 & 0.038 \text { to } 0.044 \\
0.004 \text { to } 0.014 & 0.037 \text { to } 0.044 & 0.9990 \text { to } 0.9993
\end{array}\right)
$$

The diagonal elements are, as expected, close to one and the elements for interactions between two generations are considerably smaller than the elements for interactions within one generation.

Demanding a gauge invariant theory in the case of the electroweak theory requires the introduction of four gauge fields: a massless weak isospin triplet of gauge fields $\mathbf{W}_{\mu}=\left(W_{\mu}^{1}, W_{\mu}^{2}, W_{\mu}^{3}\right)$ and a massless hypercharge singlet gauge field $B_{\mu}$. The covariant derivative for the gauge group $\mathrm{U}(1)_{\mathrm{Y}} \times \mathrm{SU}(2)_{\mathrm{L}}$, acting on the fermion fields $f$, is

$$
D_{\mu}=\partial^{\mu}+i g \mathbf{T} \mathbf{W}^{\mu}+i \frac{g^{\prime}}{2} Y B^{\mu}
$$


where $g$ is the coupling constant for $\mathrm{SU}(2)_{\mathrm{L}}$ and $g^{\prime}$ is the coupling constant for $\mathrm{U}(1)$. The matrices $\mathbf{T}$ are the generators of the group SU(2). For the right-handed fermion fields $\mathbf{T}=0$, as they do not couple to the $W^{ \pm}$. The fundamental representation of $\mathbf{T}$ for left-handed fermion fields is related to the Pauli spin matrices via $\mathbf{T}=\boldsymbol{\sigma} / 2$ :

$$
T_{1}=\frac{1}{2}\left(\begin{array}{ll}
0 & 1 \\
1 & 0
\end{array}\right), T_{2}=\frac{1}{2}\left(\begin{array}{cc}
0 & -i \\
i & 0
\end{array}\right), T_{3}=\frac{1}{2}\left(\begin{array}{cc}
1 & 0 \\
0 & -1
\end{array}\right)
$$

As mentioned above, $\mathrm{SU}(2)_{\mathrm{L}}$ and $\mathrm{U}(1)_{\mathrm{Y}}$ cannot be identified with the weak and the electromagnetic interaction respectively. For instance, the neutral gauge field $W_{\mu}^{3}$ cannot be identified with the $\mathrm{Z}^{0}$ boson, as observations show that the neutral non-QED current is not purely left-handed. The physical bosons can be identified however with linear combinations of the four fields as follows:

$$
\begin{aligned}
W_{\mu}^{ \pm} & =\frac{1}{\sqrt{2}}\left(W_{\mu}^{1} \mp i W_{\mu}^{2}\right) \\
Z_{\mu} & =-B_{\mu} \sin \Theta_{W}+W_{\mu}^{3} \cos \Theta_{W} \equiv Z^{0} \\
A_{\mu} & =B_{\mu} \cos \Theta_{W}+W_{\mu}^{3} \sin \Theta_{W} \equiv \gamma,
\end{aligned}
$$

with the weak mixing angle $\Theta_{W} \equiv \tan ^{-1}\left(g^{\prime} / g\right)$. Figure 2.3 shows the resulting basic vertices of the weak sector of the electroweak interaction. The interactions of the gauge boson with the fermions of the theory are shown. As the $\mathrm{W}$ and $\mathrm{Z}$ boson themselves carry a weak charge there are, as previously discussed in the case of QCD, also vertices between those bosons. The $\mathrm{W}$ boson also carries an electromagnetic charge which results in additional $\mathrm{W} /$ photon vertices.
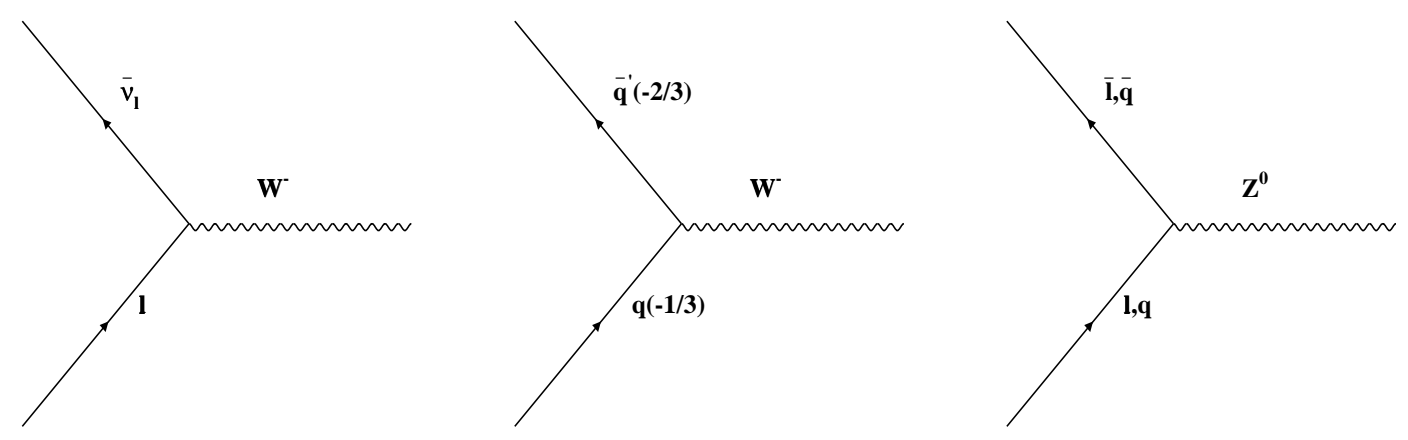

Figure 2.3: The primitive vertices for the interaction of the weak gauge bosons with the fundamental fermions.

After this unification one problem remains within the theory, relating to the identification of the $\mathrm{W}$ and $\mathrm{Z}$ bosons with the gauge bosons of the weak interaction. 
The invariance of the Lagrangian is achieved only for massless gauge fields. The photon fulfils this requirement, but the $\mathrm{Z}^{0}$ boson with a mass of about $91.2 \mathrm{GeV} / \mathrm{c}^{2}$ and the $\mathrm{W}^{ \pm}$bosons with masses of about $80.4 \mathrm{GeV} / \mathrm{c}^{2}$ certainly do not. Simply starting with a gauge invariant theory and adding the mass terms by hand destroys the renormalisability of the theory. A rather elegant way to introduce masses to the theory lies in the principle of spontaneous symmetry breaking and the Higgs mechanism [5-9].

\subsubsection{Spontaneous Symmetry Breaking and the Higgs Mech- anism}

The symmetry of a system is said to be spontaneously broken if its ground state does not exhibit the symmetry governing its dynamics. One example is the infinitely extended ferromagnet. For temperatures below the Curie temperature all spins are oriented parallel. Even though the interaction among the spins is invariant under a rotation of the system the ground state clearly is not. Once the temperature drops below the critical temperature $T_{c}$ one spin orientation is chosen out of an infinite number of possible ground states. Choosing a particular ground state produces Spontaneous Symmetry Breaking (SSB). In quantum field theory SSB occurs if the ground state of a system (the vacuum) does not share the symmetry of its Lagrangian. One feature of SSB is the appearance of massless modes, the Nambu-Goldstone bosons, or simply Goldstone bosons. The famous Higgs mechanism operates when SSB occurs in a gauge theory. In this case the Goldstone bosons are absorbed as the third degree of freedom of the massless vectorfields, which, as such, acquire mass.

In the electroweak theory SSB must break the gauge group $S U(2)_{L} \otimes U(1)_{Y}$ down to $U(1)_{e m}$, giving mass to the $\mathrm{W}$ and $\mathrm{Z}$ bosons while rendering the photon massless, as $U(1)_{e m}$ is a valid symmetry of the vacuum. This process leads to the introduction of a new field, the Higgs field. The simplest field structure that fulfils all requirements for SSB in the electroweak theory consists of two complex fields that form a doublet with respect to the weak isospin:

$$
\Phi=\left(\begin{array}{c}
\phi^{+} \\
\phi^{0}
\end{array}\right) \quad I=1 / 2, \quad \mathrm{Y}=1
$$



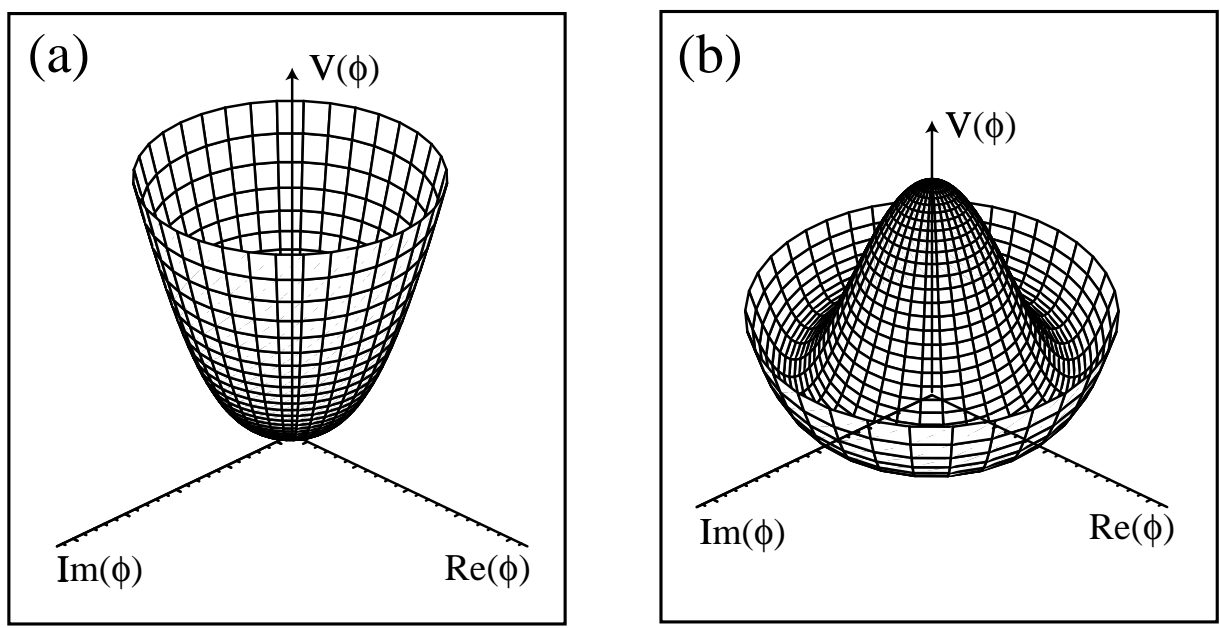

Figure 2.4: Higgs potential $V=-\mu^{2} \Phi^{\dagger} \Phi+\lambda\left(\Phi^{\dagger} \Phi\right)^{2}$ for $-\mu^{2}>0$ (a) and $-\mu^{2}<0$ (b).

The Lagrangian of the Higgs field is

$$
\left(D_{\mu} \Phi\right)^{\dagger}\left(D^{\mu} \Phi\right)-V(\Phi)
$$

with the potential

$$
V=-\mu^{2} \Phi^{\dagger} \Phi+\lambda\left(\Phi^{\dagger} \Phi\right)^{2} \text { and } \lambda>0
$$

With $-\mu^{2}<0$, the minimum of the potential $V$ is

$$
|\Phi|^{2}=\frac{\mu^{2}}{2 \lambda} \neq 0
$$

Figure 2.4 shows the potential for the cases $-\mu^{2}>0$ and $-\mu^{2}<0$.

This groundstate is degenerate through an arbitrary phase $\phi$ and thus has no preferred direction in the weak isospin space. However once a specific ground state is chosen SSB occurs. For instance choosing

$$
\phi_{0}=\frac{1}{\sqrt{2}}\left(\begin{array}{l}
0 \\
v
\end{array}\right), v=\sqrt{\frac{\mu^{2}}{\lambda}}
$$

breaks $S U(2)_{L} \otimes U(1)_{Y}$ leaving $U(1)_{e m}$ as a symmetry of the vacuum. Feynman calculus is based on perturbation theory, where fields are treated as fluctuations around the ground state. Expanding $\Phi$ around the chosen ground state yields a Lagrangian where the Goldstone bosons are absorbed by the gauge bosons fields. This Lagrangian also contains a new field, the Higgs field, and a massive scalar particle, 
the Higgs boson. Through an additional term in the complete SM Lagrangian, the Yukawa term, the fermions of the theory acquire mass via coupling to the Higgs field. As just one physical Higgs boson occurs in this theory it is often referred to as Higgs boson of the minimal Standard Model. In nature the Higgs field could be of a more complex structure with several Higgs Bosons (five in the case of a supersymmetric extension of the SM). While all couplings to bosons and fermions are known as a function of the Higgs mass, the mass itself is a free parameter of the theory. One experimentally verifiable prediction of this minimal model is the ratio of the mass of the $\mathrm{W}$ and $\mathrm{Z}$ bosons:

$$
\frac{M_{W}}{M_{Z}}=\cos \left(\theta_{W}\right)
$$

with the weak mixing angle $\theta_{W}$ as in equation 2.14. However despite all efforts undertaken to find the Higgs boson, it still eludes detection. The Higgs Hunter's Guide [10] provides detailed remarks to all features of a Higgs boson in this minimal model and also more complicated models with several Higgs bosons.

\subsection{Symmetries in the Standard Model}

Investigating symmetries of a model or a physical state can often simplify the extraction of information on the system. Part of the merit of symmetries lies in their dynamical implications. Emmy Noether published her theorem relating symmetries to conservation laws in 1918 [11]. It states that invariance under a global transformation yields a conserved quantity and vice versa.

There are several types of symmetries in the SM. Here only the discrete spacetime symmetries, namely parity, charge conjugation and time reversal are mentioned.

\subsubsection{Parity}

A parity transformation is the transformation of the space-time coordinates $x, y$ and $z$ to $-x,-y$ and $-z$ respectively. All particles are eigenstates of the parity operator. The electromagnetic interaction and the strong interaction are invariant under such a transformation; the mirror image of an electromagnetic or strong interaction is also a valid physical process of the respective interaction. Before 1956 all interactions were believed to be parity conserving. However Lee and Yang found that there was no 
actual confirmation of such an invariance in the case of the weak interaction. They suggested an experiment which was later performed by C.S. Wu et al. [12,13]. The result of this experiment showed that the weak interaction does violate parity. It was found later that the violation is indeed maximal, treating neutrinos in the massless approximation. The spin of a particle is an axial vector, i.e. it does not change direction under a parity transformation. Thus the parity operator would turn a left-handed neutrino, i.e. a neutrino where spin and momentum vector are aligned, into a right-handed neutrino where spin and momentum vector are anti-parallel. However in the massless approximation no right-handed neutrinos are observed.

\subsubsection{Charge Conjugation}

The operator for charge conjugation $\mathrm{C}$ changes a particle into its antiparticle. The name charge conjugation is slightly misleading as the operation changes all internal quantum numbers leaving only energy, momentum, mass and spin unchanged, thus also turning a neutral particle into its antiparticle. Particles that are their own antiparticle are thus eigenstates of C. Electromagnetic and strong processes are invariant under charge conjugation and yet again in the weak interaction $\mathrm{C}$ is not conserved. Charge conjugation would transform a left-handed neutrino into a left-handed antineutrino, which is not realised in nature if neutrinos are massless. However the application of the combined transformation CP turns a left-handed neutrino into a right-handed antineutrino, which does exist. This led to the proposition that the combined charge and parity transformation could be a conserved quantity for all interactions. It was proposed that maybe this is how a "mirror" process should have been defined from the start. However much to the disappointment of many people, CP was also found to be violated in the weak interaction, in the neutral kaon system. Even though there is no obvious theoretical reason for $\mathrm{CP}$ violation in the Standard Model, it can be introduced via a complex phase $\delta$ in the CKM matrix. The discovery of CP violation in the weak interaction was the true motivation for introducing the CKM matrix as a $3 \times 3$ matrix even though at the time only two quark generations were known. A $2 \times 2$ matrix would only have one real phase and no complex phase at all, while the $3 \times 3$ matrix has three real 
parameters and one complex phase. Re-expressed in terms of this complex phase and three rotational angles $\theta_{12}, \theta_{23}, \theta_{13}$ the CKM matrix can be expressed as [1]

$$
\left(\begin{array}{ccc}
c_{12} c_{13} & s_{12} c_{13} & s_{13} e^{-i \delta} \\
-s_{12} c_{23}-c_{12} s_{23} s_{13} e^{i \delta} & c_{12} c_{23}-s_{12} s_{23} s_{13} e^{i \delta} & s_{23} c_{13} \\
s_{12} s_{23}-c_{12} c_{23} s_{13} e^{i \delta} & -c_{12} s_{23}-s_{12} c_{23} s_{13} e^{i \delta} & c_{23} c_{13}
\end{array}\right)
$$

with $c_{i j}=\cos \theta_{i j}$ and $s_{i j}=\sin \theta_{i j}$.

\subsubsection{Time Reversal and the CPT Theorem}

It is difficult to test whether a process is invariant under time reversal; in the laboratory time cannot be reversed. A reaction can however run in the opposite direction and the reaction rates for both directions can be compared. It is however difficult to identify reactions with similar phase space in both directions. For the strong and electromagnetic interaction no deviation from an invariance under $\mathrm{T}$ as been observed. In the case of the weak interaction time invariance is extremely difficult to test since most processes are dominated by the strong and electromagnetic interaction. Purely weak interactions, i.e. interactions involving neutrinos, are hard to measure with high precision. Currently the only direct observation of $\mathrm{T}$ violation was reported by the CPLEAR collaboration through a measurement of the asymmetry between a $\mathrm{K}_{0}$ transforming into a $\overline{\mathrm{K}}_{0}$ and vice versa as a function of neutral-kaon decay time [14]. Theoretically one would expect $\mathrm{T}$ to be conserved for the electromagnetic and strong force and violated in the weak force. This is due to the fact that processes have to be invariant under the combined transformation $\mathrm{CPT}$ in any relativistic field theory. This statement is called the CPT theorem. One consequence of this theorem is that the mass and the lifetime of a particle equals exactly the mass and the lifetime of its antiparticle.

\subsection{B Hadron Production}

The $\Lambda_{b}$ baryons examined for their lifetime in this thesis are ultimately created through the hadronisation of $\mathrm{b}$ quarks produced in $p \bar{p}$ collisions. The cross section for $b \bar{b}$ production, i.e. the reaction rate per instantaneous luminosity, at the Tevatron reaches approximately $0.1 \mathrm{mb}$ due to the high centre-of-mass energy. Thus a peak 
luminosity of $2 \times 10^{32} \mathrm{~cm}^{-2} \mathrm{~s}^{-1}$ results in the production of up to $2 \times 10^{4} \mathrm{~b} \overline{\mathrm{b}}$ pairs every second. However the total hadronic cross section is approximately $75 \mathrm{mb}$, nearly three orders of magnitude higher, demanding a sophisticated trigger system to select the interesting events from heavy quark production. In the following sections the mechanisms for heavy quark production relevant to the Tevatron and their subsequent fragmentation are discussed.

\subsubsection{Leading Order Processes for Heavy Quark Production}

At leading order in $\alpha_{s}$ (i.e. for probabilities $\propto \alpha_{s}^{2}$ ) heavy quark $(Q)$ and antiquark $(\bar{Q})$ pairs are produced via the hard QCD 2 to 2 parton subprocesses

$$
\begin{aligned}
& \text { (a) } q+\bar{q} \rightarrow Q+\bar{Q} \\
& \text { (b) } g+g \rightarrow Q+\bar{Q} .
\end{aligned}
$$

The Feynman diagrams for the above interactions are depicted in figure 2.5.

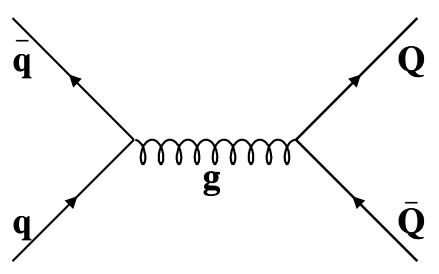

(a)

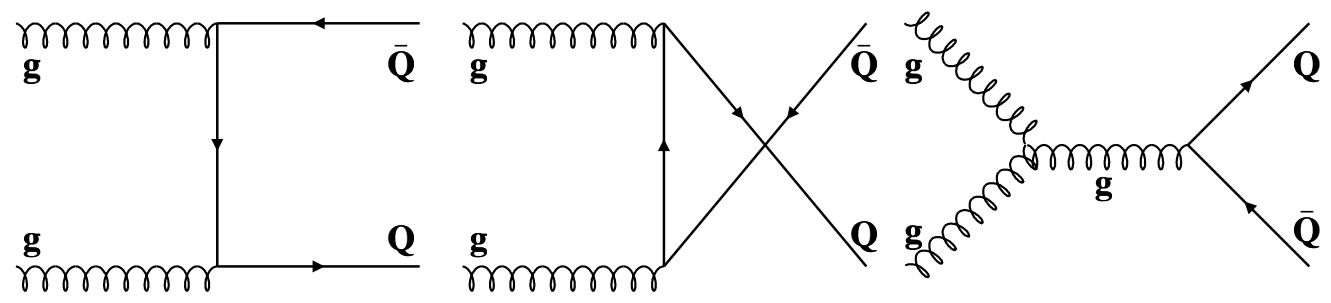

(b)

Figure 2.5: Leading order Feynman diagrams for heavy quark production. Figure (a) shows the production of a $Q \bar{Q}$ pair via the annihilation of a light quark-antiquark pair and figure (b) shows the gluon gluon fusion processes. 
To obtain the total hadro-production cross section from the above processes, the quark cross sections are convoluted with the parton distribution functions $f_{i, j}(x)$ as follows:

$$
\frac{d \sigma}{d y d \bar{y} d p_{T}^{2}} \propto \frac{1}{m_{T}^{4}(1+\cosh (\Delta y))^{2}} \times \sum_{i, j} x_{1} f_{i}\left(x_{1}\right) x_{2} f_{j}\left(x_{2}\right) \bar{\sum}|M(i j \rightarrow Q \bar{Q})|^{2}
$$

$m_{T}$ is the transverse mass $\sqrt{m_{Q}^{2}+p_{T}^{2}}$ and $y$ and $\bar{y}$ are the rapidity of the heavy quark and antiquark respectively. $\bar{\sum}$ indicates averaging(summation) over initial(final) colour and spin states. Due to the cosh term in the denominator the $Q \bar{Q}$ pairs tend to be produced with similar rapidities. Figure 2.6 shows the production cross section for $\mathrm{b}, \mathrm{c}$ and $\mathrm{t}$ quark pairs in $p \bar{p}$ collisions.

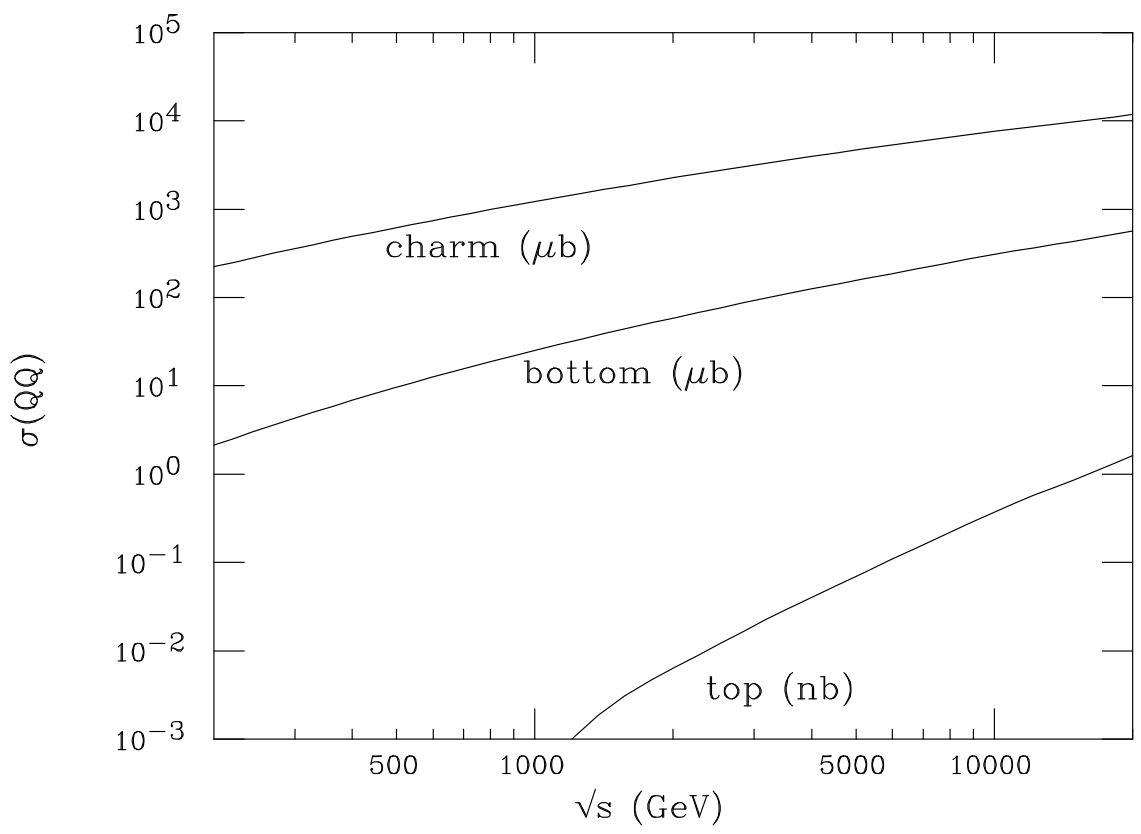

Figure 2.6: Total production cross section for heavy quark pairs in $p \bar{p}$ collisions [15]. Note the different scale for top quarks (nb) compared to charm and bottom quarks $(\mu \mathrm{b})$.

\subsubsection{Next to Leading Order Processes}

The leading order processes detailed above, together with contributions from initial and final state gluon radiation, are called flavour creation. Examples of NLO contributions to the flavour creation process are shown in figure 2.7. 


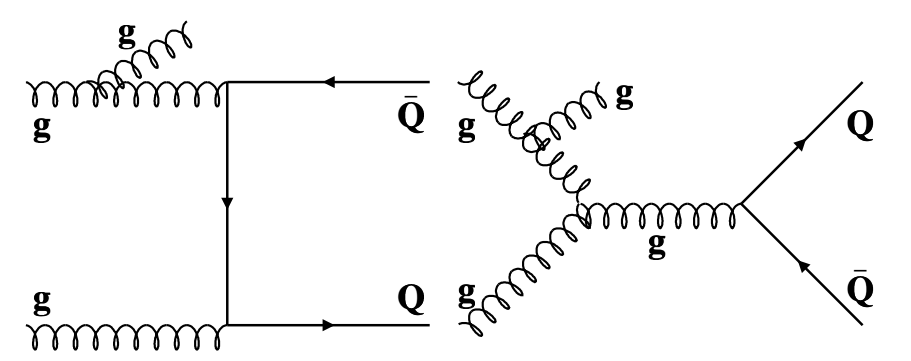

(a) real gluon emission

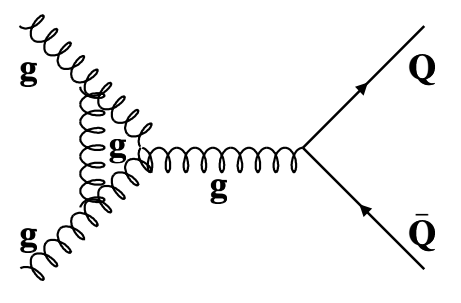

(b) virtual emission

Figure 2.7: Example Feynman diagrams for NLO contributions to flavour creation.

Additionally to these diagrams there is a further set of NLO processes relevant for hadro-production at the Tevatron, which are depicted in figure 2.8 (b). The first Feynman diagram of figure 2.8 (b) is often called gluon splitting, while the remaining two diagrams are sometimes categorised as graphs for flavour excitation. Figure 2.8 (a) shows the process which is more commonly called flavour excitation. The reason for this nomenclature lies in the fact that diagram (a) is already present as a subdiagram in the latter two diagrams of (b). Because of this, diagram (a) should not be included when considering the total cross section.

\subsubsection{Quark Fragmentation}

In this thesis the heavy quark of interest is the b quark. After the heavy quarkantiquark pair is created the quarks can rid themselves of part of their momentum through the emission of gluons, before they eventually form the observable hadrons. This hadronisation is a long distance process with small momentum transfer in which perturbative QCD is not applicable. Thus this part of hadron creation is described through phenomenological models. One of those models, the string model, is based on the dynamics of a relativistic string that represents the colour flux stretched between the initial $b \bar{b}$ pair. When the $b \bar{b}$ pair moves apart, the string breaks up, usually creating a light $q \bar{q}$ pair out of the intense colour field. The string breaking may be parametrised by certain fragmentation models, one of which is discussed below. The newly created $q \bar{q}$ pair can have different flavours resulting in differently flavoured $\mathrm{B}$ hadrons. The fractions with which the initial $\mathrm{b}$ quark combines to 


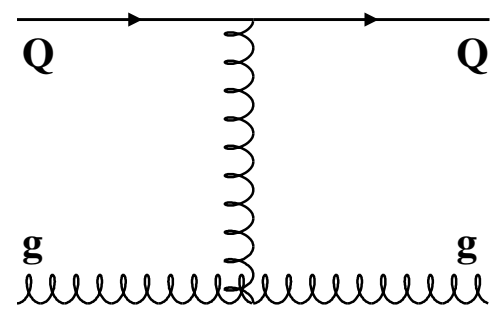

(a)
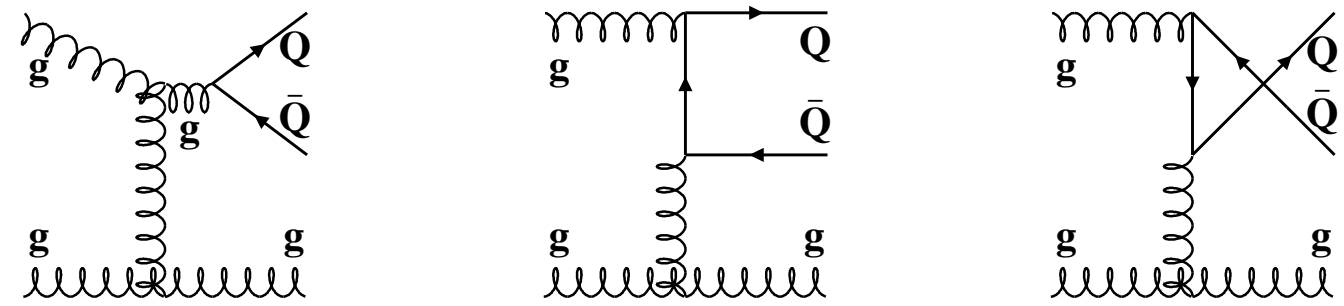

(b) Feynman graphs with spin one exchange in the t-channel

Figure 2.8: Figure (a) represents what is most commonly known as flavour excitation. The first graph in figure (b) represents gluon splitting which is a relevant mechanisms for heavy quark production at the Tevatron. The remaining graphs in figure (b) also contribute significantly to the b-quark cross section and are often referred to as flavour excitation as they include figure (a) as a subdiagram.

form various weakly decaying B mesons as well as the $\Lambda_{b}$ baryon were measured at CDF [16] and are shown in table 2.3. The newly produced heavy flavoured hadron retains a large fraction of the momentum of the primordial heavy quark. The heavier the quark, the higher the momentum fraction is found to be. There exist several different parametrisations for the phenomenologically determined non-perturbative part of the fragmentation process. One of the better known parametrisations is that by Peterson, Schlatter, Schmitt and Zerwas [17] known generally as the Peterson

\begin{tabular}{|c|c|cc|}
\hline B Hadron & Quark Content & Production Fraction assuming $f_{u}=f_{d}$ \\
\hline$B^{+}$ & $\bar{b} u$ & $f_{u}=0.375 \pm 0.023$ \\
\hline$B^{0}$ & $\bar{b} d$ & $f_{d}=0.375 \pm 0.023$ \\
\hline$B_{s}$ & $\bar{b} s$ & $f_{s}=0.160 \pm 0.044$ \\
\hline$\Lambda_{b}$ & $b u d$ & $f_{\text {baryon }}=0.090 \pm 0.029$ \\
\hline
\end{tabular}

Table 2.3: B quark fragmentation fractions for weakly decaying $B$ hadrons from $p \bar{p}$ collisions [16]. Dropping the requirement $f_{u}=f_{d}$ the ratio $f_{d} / f_{u}$ is found to be $0.84 \pm 0.16$. 
fragmentation function. Here the function is obtained by assuming that the energy difference of the system before and after fragmentation is inversely proportional to the transition amplitude. For a heavy quark $Q$ with momentum $P$ that fragments into a heavy hadron $H=Q \bar{q}$ with momentum $z P$ and the remaining light quark $q$ this energy difference is

$$
\Delta E=E_{Q}-E_{H}-E_{q}=\sqrt{m_{Q}^{2}+P^{2}}-\sqrt{m_{H}^{2}+z^{2} P^{2}}-\sqrt{m_{q}^{2}+(1-z)^{2} P^{2}} .
$$

Assuming $m_{Q} \approx m_{H}$ this results in

$$
\Delta E=P\left[\sqrt{\frac{m_{Q}^{2}}{P^{2}}+1}-\sqrt{\frac{m_{Q}^{2}}{P^{2}}+z^{2}}-\sqrt{\frac{m_{q}^{2}}{P^{2}}+(1-z)^{2}}\right] .
$$

Expanding around $x=m_{Q}^{2} / P^{2}$ at $x=0$ gives for $P \gg m_{Q}$

$$
\Delta E=\frac{m_{Q}^{2}}{2 P}\left[1-\frac{1}{z}-\frac{\epsilon_{Q}}{1-z}\right]
$$

where $\epsilon_{Q}=m_{q}^{2} / M_{Q}^{2}$. The complete Peterson fragmentation function for the fragmentation of a heavy quark $Q$ into a heavy hadron $H$ is then obtained by squaring the amplitude and adding a factor $1 / z$ for longitudinal phase space

$$
D(z)=\frac{N_{H}}{z} \frac{1}{\left[1-\frac{1}{z}-\frac{\epsilon_{Q}}{1-z}\right]^{2}} .
$$

The normalisation factor $N_{H}$ is obtained through a summation over all hadrons that contain the heavy quark $\mathrm{Q}$. The parameter $\epsilon$, although proportional to $m_{q}^{2} / m_{Q}^{2}$, is a free parameter of the fragmentation function and is determined experimentally. The value of $\epsilon$ depends on the order of the perturbative calculation that precedes the non-perturbative part of the fragmentation. For the leading-log approximation, $\epsilon_{c}$ and $\epsilon_{b}$ are found to be approximately 0.05 and 0.006 respectively [1]. The Peterson function for those values is displayed in figure 2.9. It can be seen that the momentum fraction for the heavier $\mathrm{b}$ quark is indeed larger than that for the $\mathrm{c}$ quark. 


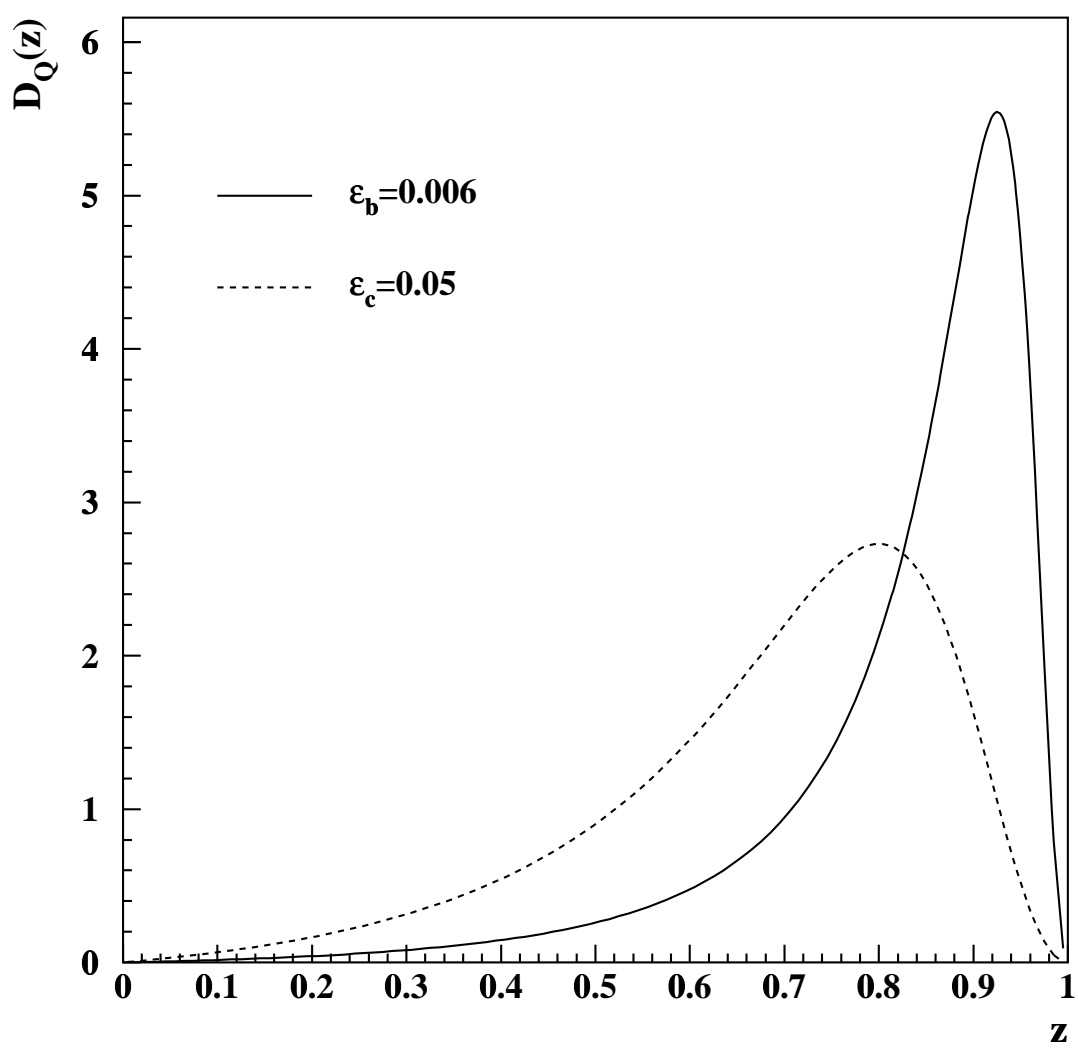

Figure 2.9: Peterson fragmentation function for b quarks $\left(\epsilon_{b}=0.006\right.$, solid curve) and $c$ quarks $\left(\epsilon_{c}=0.05\right.$, dashed curve). As expected the curve for $\mathrm{b}$ quarks is peaked at higher $\mathrm{z}$ values compared to the curve for the lighter $\mathrm{c}$ quarks.

\subsection{Decay of Heavy Hadrons}

A hadron is considered heavy if it contains a charm or a bottom quark (the top quark decays before it can hadronise), that is, if one of its constituent quarks has a mass that is large compared to the scale of QCD, $\Lambda_{Q C D}$, which is $200-300 \mathrm{MeV}$. The magnitude of the lifetime of such hadrons is governed both by the quark-quark couplings $-\mathrm{V}_{\mathrm{cb}}$ and $\mathrm{V}_{\mathrm{ub}}$ in the case of bottom hadron decays and $\mathrm{V}_{\mathrm{cs}}$ in the case of charm hadron decays - and by the decay dynamics. 


\subsubsection{The Spectator Model}

In the naive spectator model it is assumed that the light quarks in a heavy hadron do not affect the decay of the heavy quark; the lifetimes of weakly decaying hadrons that contain the same heavy quark are thus equal in this model, regardless of the flavour of the accompanying quarks. Figure 2.10 shows the Feynman diagrams for the spectator decay of bottom mesons (a) and bottom baryons (b). Following the

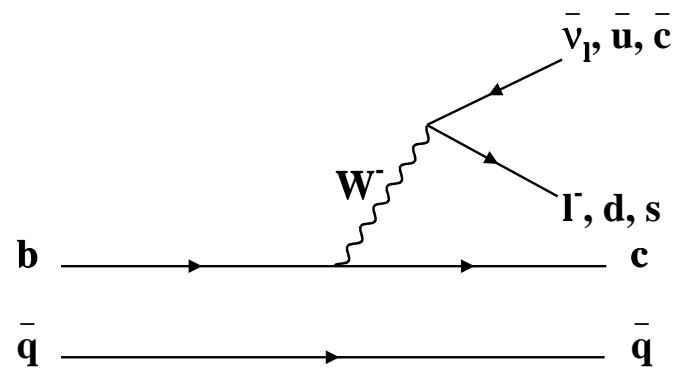

(a)

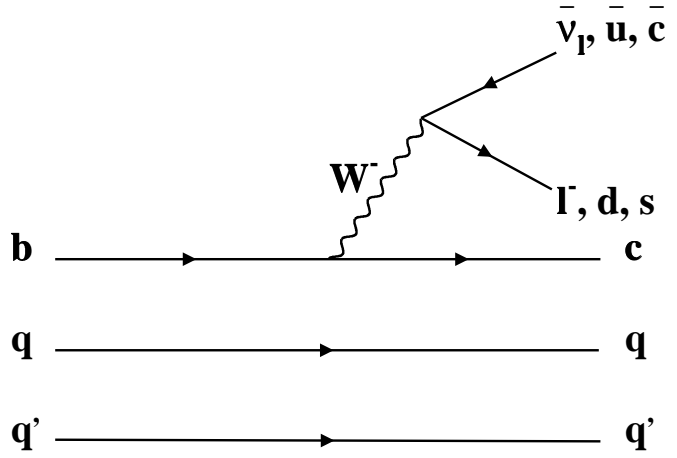

(b)

Figure 2.10: Figure (a) shows the spectator diagram for the decay of mesons containing a $b$ quark; the $b$ quark decays weakly, while the additional antiquark $\bar{q}$ is a spectator to the decay process. Figure (b) shows the spectator diagram for $b$ baryon decay.

muon decay, the spectator model can give a rough estimate of the expected lifetimes for charm and bottom hadrons. In muon decays the virtual W-boson decays to e $\nu_{\mathrm{e}}$ since that is the only kinematically allowed channel. In charm decays however the Wboson has five possibilities to decay ( $\mathrm{e} \nu_{\mathrm{e}}, \mu \nu_{\mu}$ and $\mathrm{u} \overline{\mathrm{d}} \times 3$ colours). In bottom decays the number of possible decay products increases to nine $\left(\mathrm{e} \nu_{\mathrm{e}}, \mu \nu_{\mu}, \tau \nu_{\tau}, \mathrm{u} \overline{\mathrm{d}}\right.$ and $\mathrm{s} \overline{\mathrm{c}}$, again with three colour combinations for the last two possibilities). Furthermore the quark-quark couplings and the mass of the decaying particle must be adjusted when starting from the muon decay formula. With these adjustments the lifetime of bottom hadrons can be estimated as:

$$
\Gamma_{b} \approx \Gamma_{\mu} \times \frac{9 V_{c b}^{2} M_{b}^{5}}{M_{\mu}^{5}} \Rightarrow \tau_{b}=\frac{1}{\Gamma_{b}} \approx 1.3-1.7 \mathrm{ps},
$$

with $\mathrm{M}_{\mathrm{b}}=4.2 \mathrm{GeV} / \mathrm{c}^{2}$ and $\mathrm{V}_{\mathrm{cb}}=0.038-0.044$. It is apparent, particularly due to the uncertainty in the quark mass, which enters the calculation to the fifth power, and 
due to the uncertainty in the measurement of $V_{c b}$, that this is only a very rough estimate.

Soon after the simple spectator model was proposed it became apparent, particularly in the charm sector, that other diagrams have to be included in the description of decays of heavy hadrons to explain the measured lifetime differences between hadrons containing different spectator quarks. The first indication were measurements of the lifetime of the $D^{+}$and the $D^{0}$ meson which yielded $\tau\left(D^{+}\right) / \tau\left(D^{0}\right) \approx$ 2.5. Table 2.4 lists the current results for lifetime measurements of various $\mathrm{b}$ and c hadrons, which are also shown in figure 2.11. The necessity for the inclusion of non-spectator diagrams is made obvious by the spread of the measured lifetimes.

\begin{tabular}{|c|c|c|c|c|}
\hline \multicolumn{2}{|c|}{ Charm Hadrons } & & \multicolumn{2}{c|}{ Bottom Hadrons } \\
\hline Hadron & lifetime $[\mathrm{ps}]$ & & Hadron & lifetime $[\mathrm{ps}]$ \\
\hline$D^{+}$ & $1.051 \pm 0.013$ & & $B^{+}$ & $1.674 \pm 0.018$ \\
\hline$D^{0}$ & $0.412 \pm 0.003$ & & $B^{0}$ & $1.542 \pm 0.016$ \\
\hline$D_{s}^{+}$ & $0.490 \pm 0.009$ & & $B_{s}^{+}$ & $1.461 \pm 0.057$ \\
\hline$\Lambda_{c}^{+}$ & $0.200 \pm 0.006$ & & $\Lambda_{b}^{0}$ & $1.229 \pm 0.080$ \\
\hline$\Xi_{c}^{+}$ & $0.442 \pm 0.026$ & & $\Xi_{b}$ & $1.39_{-0.28}^{+0.34}$ \\
\hline$\Xi_{c}^{0}$ & $0.098_{-0.015}^{+0.023}$ & & \multicolumn{3}{|c|}{} \\
\hline
\end{tabular}

Table 2.4: Lifetimes of various charm and bottom hadrons [1]. The $\Xi_{b}$ lifetime average is based on studies of an excess of events containing same sign $\Xi$-l pairs in jets by the ALEPH [18] and the DELPHI [19] Collaboration.
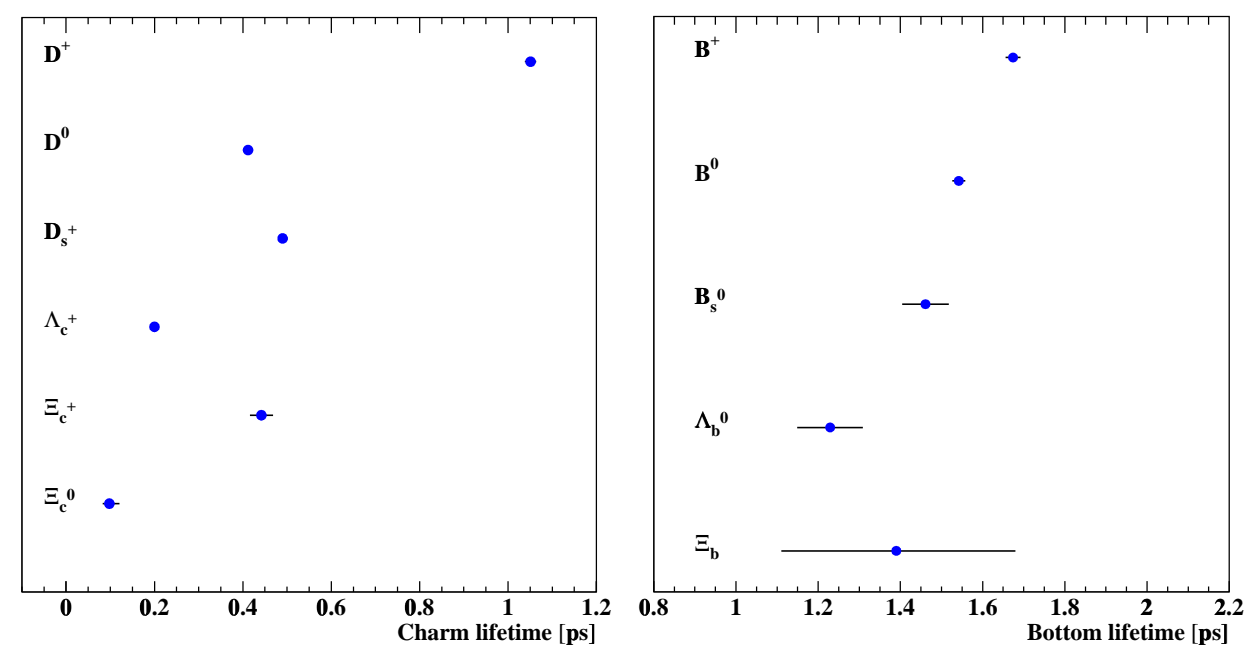

Figure 2.11: Lifetimes of charm (left) and bottom hadrons (right). 


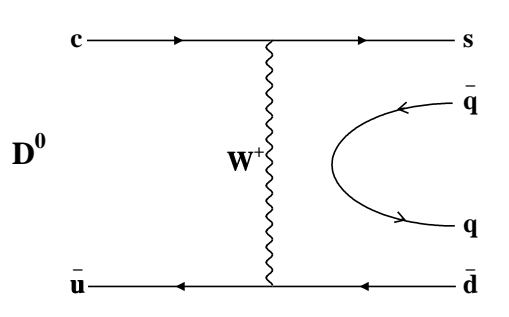

(a) Weak Annihilation

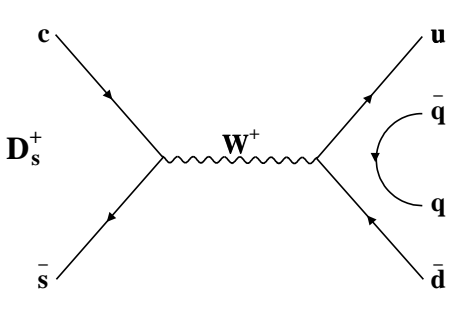

(b) W-Scattering

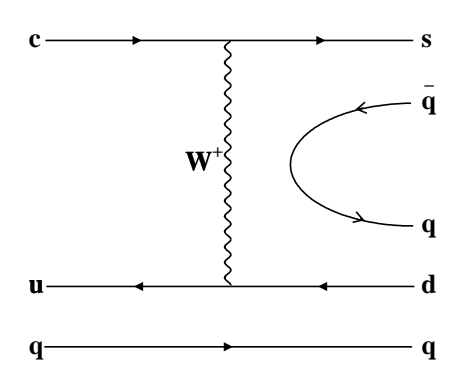

\section{(a)}




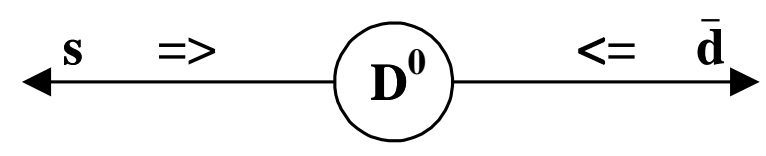

Figure 2.13: Decay of a $D^{0}$ meson ( $\left.\operatorname{spin} 0\right)$ in the $D^{0}$ restframe. The s and $\overline{\mathrm{d}}$ quark are here left-handed which causes helicity suppression for this decay.

with spin $=1 / 2$ from quark model predictions, the spin assignment causes no helicity suppression.

A second contribution to lifetime differences comes from interference effects between decay diagrams, referred to as Pauli Interference (PI). Figure 2.14 shows the diagrams for PI for the case of a $B^{-}$and a $B^{0}$ meson. The upper diagram is in each case an external spectator diagram, while the lower diagram represents the internal spectator decay. In the case of the $B^{-}$both such diagrams yield the same final state, while in the case of the $B^{0}$ the final states differ. The PI turns out to be destructive, which again prolongs the lifetime of the $B^{+}\left(D^{+}\right)$over the lifetime of the $B^{0}\left(D^{0}\right)$, and is thought to be the dominant factor leading to the lifetime differences.

However these phenomenological considerations can yield at best a hierarchy of lifetimes such as

$$
\tau\left(D^{+}\right)>\tau\left(D^{0}\right) \approx \tau\left(D_{s}^{+}\right)>\tau\left(\Lambda_{c}^{+}\right)
$$
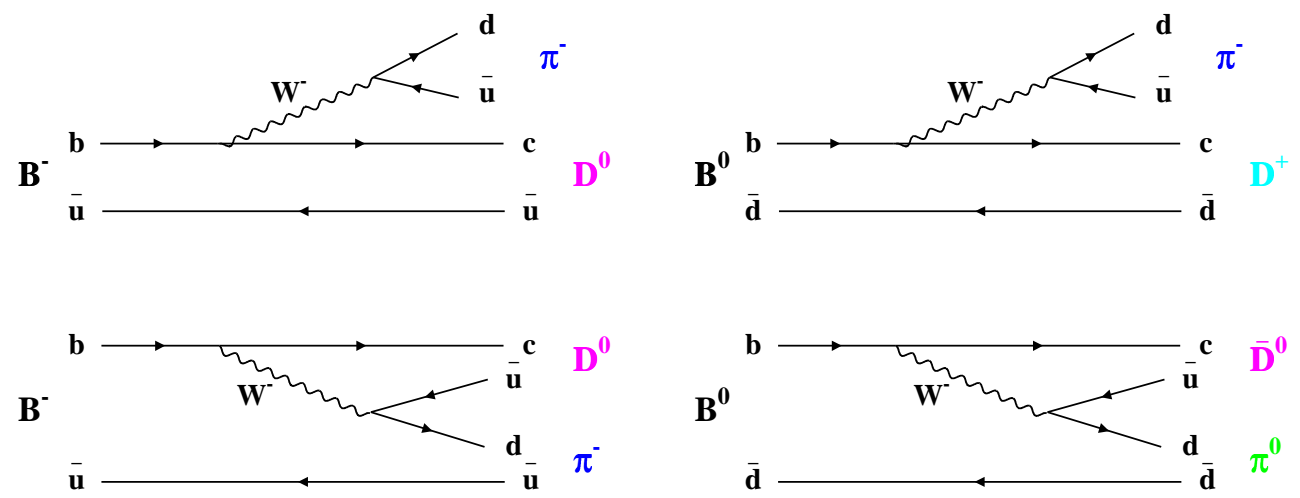

Figure 2.14: Shown are the external (top) and internal (bottom) spectator diagrams for the decay of $B^{-}$mesons and $B^{0}$ mesons on the left-hand side and on the right hand side respectively. In the case of the decay of a $B^{-}$meson the final states contain the same particles, which is known as Pauli Interference. 
They cannot indicate how the corrections scale with the heavy quark mass for instance, although the naive assumption is that corrections are smaller for the heavier bottom hadrons; by how much is unclear. Today however there exists a theoretical apparatus, the heavy quark theory, that enables concrete predictions for lifetime ratios that can be tested experimentally.

\subsubsection{Heavy Quark Theory}

Computing weak decays of heavy hadrons is a difficult task owing to the non-trivial interplay of strong and electroweak forces. Unlike in the case of electroweak processes, all orders of perturbation theory have to be considered in QCD. Various theoretical approaches are today available for the calculation of a number of aspects of heavy quark physics. Inclusive b decays are treated within the framework of the heavy quark expansion (HQE), which is basically an expansion in inverse powers of the heavy quark mass; as $m_{b} \gg \Lambda_{\mathrm{QCD}}$ the inverse quark mass provides a useful expansion parameter. The HQE is based on an operator product expansion (OPE), a method to disentangle long and short distance contributions to the decay amplitudes: a modern version of the Fermi theory of the weak interaction.

The starting point in the HQE is the total decay rate $\Gamma_{H}$ of a heavy hadron:

$$
\Gamma_{H}=\frac{1}{2 m_{H}}\langle H|\mathbf{T}| H\rangle \equiv\langle T\rangle
$$

The transition operator $\mathbf{T}$,

$$
\mathbf{T}=\operatorname{Im} i \int d^{4} x T H_{e f f}(x) H_{e f f}(0)
$$

is the imaginary (absorptive) part of the forward scattering amplitude $\mathrm{H} \rightarrow \mathrm{H}$ under the action of the effective weak Hamiltonian $H_{\text {eff }}$ [20]. The application of OPE results in a series of local operators. The coefficients of those operators are suppressed by increasing powers of $1 / \mathrm{m}_{\mathrm{b}}$. A combination with heavy quark effective theory yields then the following important results:

- $\Gamma_{H}=\Gamma_{b}$ at leading order in the HQE. For the first time the simple spectator model has its justification from heavy quark theory. At leading order the total decay rate of a b flavoured hadron equals the rate of the free b-quark decay regardless of the additional constituent quarks. 
- The first correction term appears at order $1 / m_{b}^{2}$ and concerns the recoil motion of the heavy quark and interactions of the light hadronic cloud with the heavy quark spin. The latter only contributes to the decay of B mesons. For the $\Lambda_{b}$ baryon this term is zero.

- The effects from non spectator diagrams such as WA and PI are present through contributions at order $1 / m_{b}^{3}$. They mainly distinguish between $B_{d}$ and $B_{u}$ mesons (and the equivalent in the charm meson sector).

In this way heavy quark theory yields with the HQE, for the first time, theoretical predictions for lifetime ratios which can be tested experimentally.

\subsubsection{Motivation for the Measurement of the $\Lambda_{b}$ Lifetime}

Table 2.5 shows the theoretical predictions for lifetime ratios of bottom hadrons [21] together with the world averages of current experimental results for the same ratios [21].

\begin{tabular}{|c|c|c|}
\hline Ratio & Theoretical Prediction [21] & World Average [21] \\
\hline$\tau\left(B^{+}\right) / \tau\left(B_{d}\right)$ & $1.06 \pm 0.02$ & $1.073 \pm 0.014$ \\
\hline$\tau\left(B_{s}\right) / \tau\left(B_{d}\right)$ & $1.00 \pm 0.01$ & $0.949 \pm 0.038$ \\
\hline$\tau\left(\Lambda_{b}\right) / \tau\left(B_{d}\right)$ & $0.90 \pm 0.05$ & $0.798 \pm 0.052$ \\
\hline
\end{tabular}

Table 2.5: Theoretical predictions and experimental results for the ratio of exclusive lifetimes of $\mathrm{b}$ hadrons.

The experimental values agree reasonably well with the theoretical predictions, however the lifetime of the $\Lambda_{b}$ baryon is a little shorter than expected from theory. Revised theoretical calculations could not account for the short lifetime as measured by the CERN experiments ALEPH, DELPHI and OPAL, and the CDF experiment during Run I at the Tevatron. However the experimental errors are still large and cannot rule out the validity of the theoretical approach. The error of the lifetime measurement is still statistically dominated.

The Tevatron is currently the only accelerator that can produce $\Lambda_{b}$ baryons and thus provides a unique opportunity to throw further light into this area. 

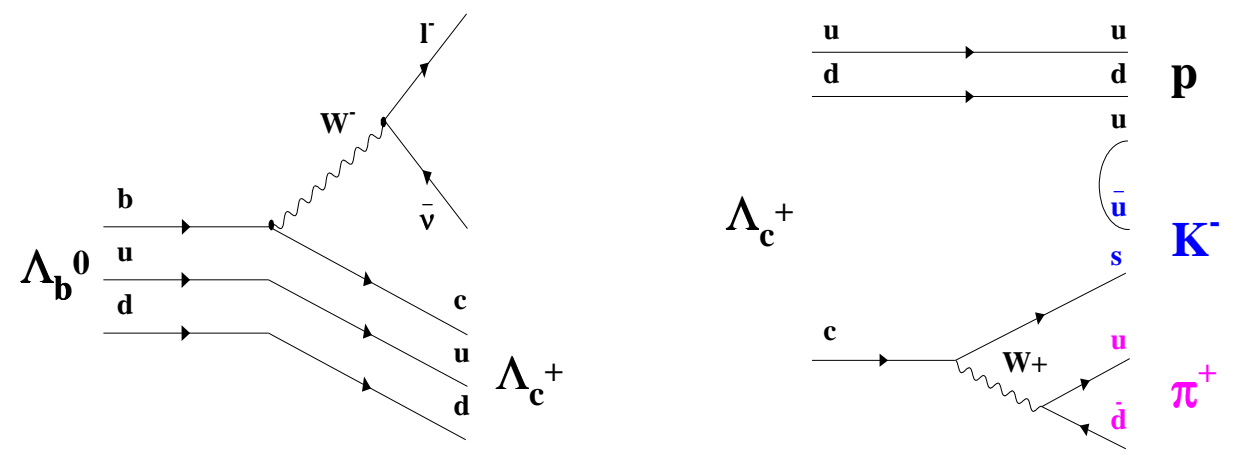

Figure 2.15: Feynman diagrams for the decays $\Lambda_{b}^{0} \rightarrow \Lambda_{c}^{+} l^{-} \bar{\nu}_{l}$ and $\Lambda_{c}^{+} \rightarrow p K^{-} \pi^{+}$.

There are two main decay modes available for this lifetime measurement

- $\Lambda_{b}^{0} \rightarrow J / \psi \Lambda, \quad \Gamma_{i} / \Gamma=(4.7 \pm 2.8) \times 10^{-4}[22]$

- $\Lambda_{b}^{0} \rightarrow \Lambda_{c}^{+} l^{-} \bar{\nu}_{l}, \quad \Gamma_{i} / \Gamma=9.2 \pm 2.1 \%[22]$.

The advantage of the former mode is that it is fully reconstructed, while the latter contains an undetectable neutrino. Furthermore the decay of the $J / \psi$ to two muons has a very clean signature. However the branching fraction for the first decay is several orders of magnitudes lower so that it was not considered up until the end of Tevatron Run I. This thesis presents a measurement of the lifetime of the $\Lambda_{b}$ baryon in its semileptonic channel $\Lambda_{b}^{0} \rightarrow \Lambda_{c}^{+} \mu^{-} \bar{\nu}_{\mu}$, with the subsequent decay $\Lambda_{c}^{+} \rightarrow p K^{-} \pi^{+}$. Figure 2.15 shows the Feynman diagrams for both decays.

The reconstruction and selection of those events is described following chapter 3 which introduces the CDF detector, with which the data for this analysis were recorded. The methodology of the lifetime measurement and the measurement of the lifetime itself are subsequently discussed. The results are presented after consideration of the systematic errors. 


\section{Chapter 3}

\section{The Experimental Apparatus}

The Collider Detector at Fermilab (CDF) is one of two high energy physics experiments designed to study interactions of protons $(\mathrm{p})$ and anti-protons $(\overline{\mathrm{p}})$ provided by the Tevatron collider at the Fermi National Accelerator Laboratory (FNAL), located about $60 \mathrm{~km}$ west of Chicago in Illinois.

\subsection{The Accelerator Complex}

The Tevatron collider is the final stage in a chain of accelerators that create and gradually accelerate protons and anti-protons which are then brought to collision at two designated interaction points around which the general purpose detectors CDF and $\mathrm{D} \varnothing$ are built. It provided first collisions in 1985 with a centre-of-mass energy $\sqrt{\mathrm{s}}$ of $1.8 \mathrm{TeV}$. Between 1996 and 2001 the accelerator complex experienced a major upgrade and currently provides collisions at a centre-of-mass energy of $1.96 \mathrm{TeV}$ referred to as Tevatron Run II. Figure 3.1 shows an aerial view of Fermilab. The actual accelerators are found below the surface, the general layout however can clearly be seen due to the roads and cooling water ponds following the ring at ground level.

Figure 3.2 shows a schematic view of the accelerators which are briefly described in the following. For further details on the Tevatron Run II see Moore [23] and the reference therein. Edward Wilson [24] provides a good introduction to the physics of accelerators. 


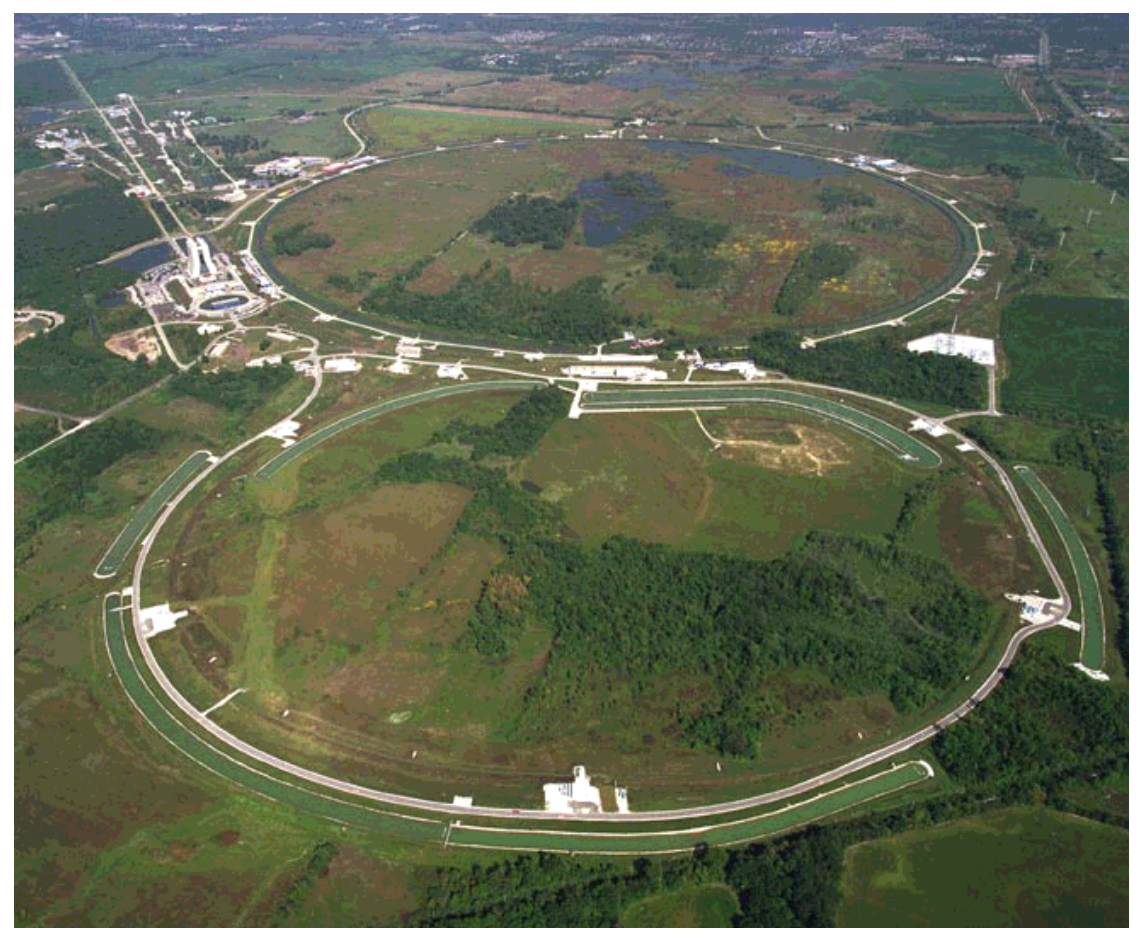

Figure 3.1: Aerial view of the accelerator complex at the Fermi National Accelerator Laboratory. The picture shows the roads and supply systems above the Tevatron and the Main Injector (upper and lower ring respectively).

\section{The Proton Source}

The origin of the proton beam is a negative ion hydrogen source. Electrons are added to hydrogen atoms by collision with the cathode of a magnetron, thereby creating hydrogen ions which are electrostatically accelerated to $750 \mathrm{keV}$ in a CockcroftWalton pre-accelerator and fed into a linear accelerator, the Linac. The Linac comprises an Alvarez type linear accelerator, built in 1971, which accelerates the ions to an energy of $116 \mathrm{MeV}$ and a more recent (1993) side-coupled cavity linear accelerator, replacing part of the old Alvarez type Linac. The ions leave this system with an energy of $400 \mathrm{MeV}$. Upon injection into the next accelerator the $\mathrm{H}^{-}$ions pass a stripper foil to strip of all their electrons. This accelerator, the Booster, is the first synchrotron in the chain. It has a circumference of $475 \mathrm{~m}$ and accelerates the protons to $8 \mathrm{GeV}$ in 0.033 seconds. From here they are passed on to the main injector (lower ring in figure 3.1), a $150 \mathrm{GeV}$ synchrotron serving several purposes. Its operation modes include accelerating protons and anti-protons from $8 \mathrm{GeV}$ to $150 \mathrm{GeV}$, coalescing several proton bunches into a more intense single bunch and 


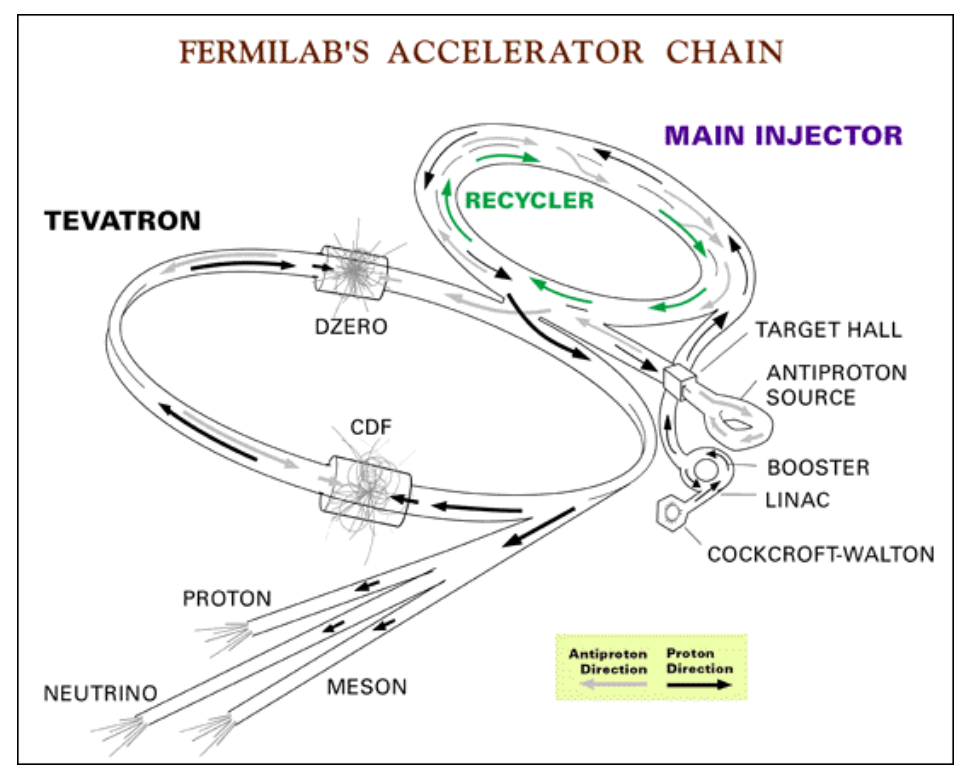

Figure 3.2: Schematic view of the accelerator complex at the Fermi National Accelerator Laboratory.

providing bunches of up to $5 \times 10^{12}$ protons for the anti-proton production, which is described in the following.

\section{The Anti-Proton Source}

Colliding protons and anti-protons as compared to protons and protons has a major advantage even though anti-protons are more difficult to produce in sufficient numbers. Being of opposite charge both particle types can be accelerated in a single ring if they circle the ring in opposite directions. This reduces the cost of an accelerator significantly since only one set of expensive superconducting magnets is needed. Additionally, for collisions at $\sqrt{\mathrm{s}}$ of up to $3 \mathrm{TeV}$ the production rate for a number of interesting processes is higher in p $\bar{p}$ collisions. At higher energies such as $\sqrt{s}=14$ $\mathrm{TeV}$, which will be provided by the Large Hadron Collider (LHC) at CERN from 2007 onwards, the rates for pp and $\mathrm{p} \overline{\mathrm{p}}$ collisions are expected to become similar. Figure 3.3 shows the general layout of the anti-proton source at the Tevatron. Every 1.47 seconds the main injector provides a $16 \mu$ s long bunch of up to $5 \times 10^{12}$ protons with an energy of $120 \mathrm{GeV}$. These protons are directed onto a $8 \mathrm{~cm}$ long nickel target. A lithium lens focuses secondary particles that emerge from these collisions with approximately $8 \mathrm{GeV}$. A pulsed dipole magnet (located at the bend after the 
anti-proton production target in figure 3.3) then deflects the anti-protons towards the debuncher while unwanted particles with the wrong charge-to-mass ratio are filtered out of the beam and are collected by a graphite-core beam-dump.

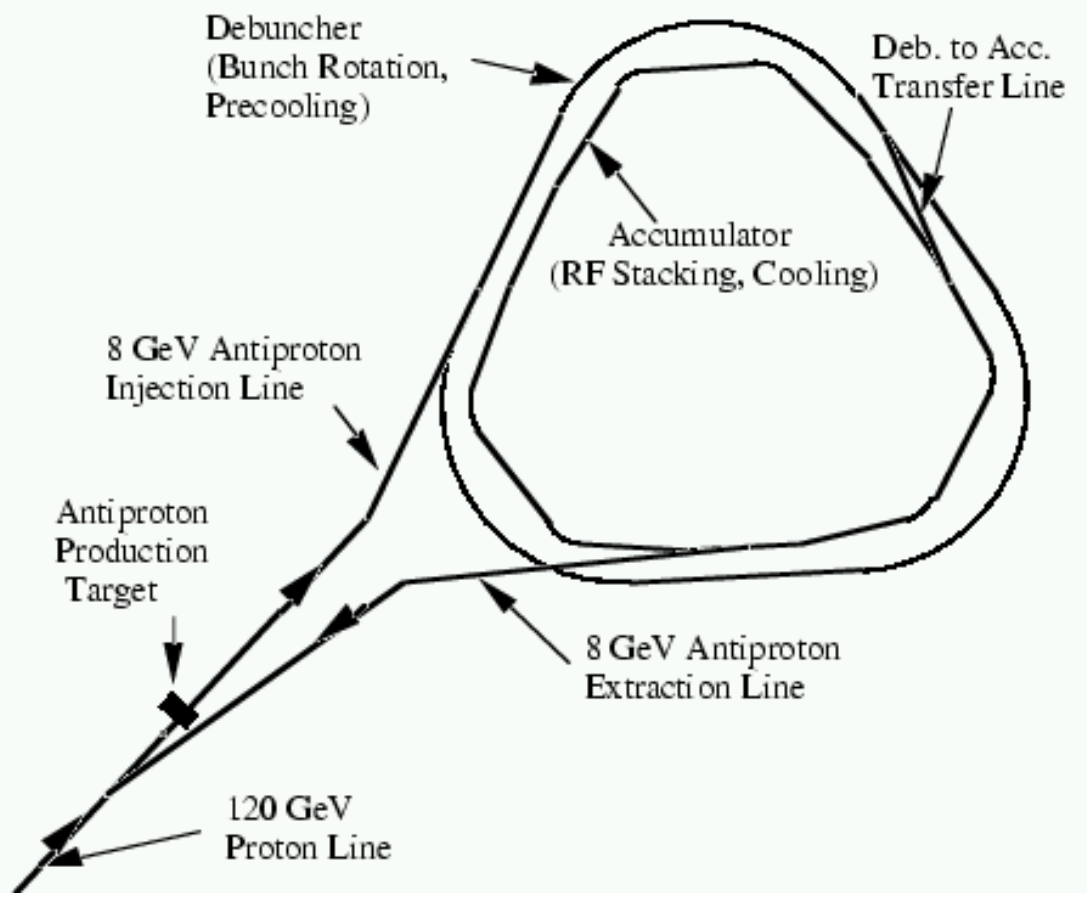

Figure 3.3: Layout of the anti-proton source.

The selected anti-protons still have a spread in energy difficult to accept for downstream accelerators. Their time spread is however small due to the fact that the protons used for their creation were grouped into bunches with narrow time spread. In the debuncher ring, an $8 \mathrm{GeV}$ synchrotron with $500 \mathrm{~m}$ circumference, this narrow time spread is transformed into a narrow energy spread. More energetic particles travel at the outside of the debuncher ring and will arrive at the radio frequency cavities later than less energetic particles which travel at the inside. Thus the particles see different phases, more energetic anti-protons are decelerated while less energetic ones are accelerated. This results, after a sufficient number of cycles, in a larger time spread but also the desired narrow energy spread. This process takes only approximately $100 \mathrm{~ms}$. The main injector delivers $\overline{\mathrm{p}}$ bunches only every $1.5 \mathrm{~s}$, leaving some time for stochastic cooling to decrease the transverse beam size before the anti-protons are transferred to the accumulator ring. The accumulator, 
also an $8 \mathrm{GeV}$ synchrotron located within the debuncher ring with a circumference of $475 \mathrm{~m}$, is used to accumulate large numbers of anti-protons using further stochastic cooling. This is achieved using feedback. Pick-up electrodes measure whether a particle deviates from its designated path and, if it does, a kicker linked to that pick-up electrode will correct this particle's orbit further down its path.

Once the beam is cooled and stacked it is transfered to the main injector, where both protons and anti-protons are accelerated to $150 \mathrm{GeV}$ and then passed onto the final accelerator, the Tevatron.

\section{The Tevatron}

The Tevatron is a p p collider of approximately $6 \mathrm{~km}$ circumference. Being of opposite charge the protons and anti-protons can be accelerated within the same beam pipe. In Run II they are accelerated to an energy of $980 \mathrm{GeV}$ (compared to $900 \mathrm{GeV}$ in Run I). Superconducting magnets with a field strength of 4.2 Tesla keep the particles on their circular path. The main difference from Run I is that there are now 36 bunches of protons and anti-protons each in the ring compared to six in Run I. This results in a bunch spacing of $396 \mathrm{~ns}$ compared to $3500 \mathrm{~ns}$. Once the bunches reach their final energy they are brought to collision within the detectors CDF and DØ.

In contrast to fixed target experiments, colliders have the advantage that the whole beam energy is available to create new massive particles. The Lorentzinvariant form for the total centre-of-mass energy of two colliding particles with masses $m_{1}$ and $m_{2}$ is

$$
E_{c m}=: \sqrt{s}=\sqrt{\left(E_{1}+E_{2}\right)^{2}-\left(\overrightarrow{p_{1}} \overrightarrow{p_{2}}\right)}
$$

Considering head-on collisions and particles with masses negligible compared to their energies this leads to:

$$
\sqrt{s_{\text {collider }}} \approx \sqrt{4 E_{1} E_{2}} \stackrel{E_{1}=E_{2}}{=} 2 E_{1} .
$$

In the case of the Tevatron, $E_{1}$ and $E_{2}$ are each $980 \mathrm{GeV}$, resulting in a centre-ofmass energy of $1.96 \mathrm{TeV}$. At a fixed target experiment however (again neglecting the mass $m_{1}$ of the accelerated particle) we have:

$$
\sqrt{s_{\text {fixed target }}} \approx \sqrt{m_{2} E_{\text {beam }}} .
$$


For colliders, $\sqrt{s}$ is proportional to the beam energy, while at fixed target experiments the available energy grows only with the square root of the energy of the incoming beam. Additionally in a collider the beam is not lost after one collision and all particles that did not interact keep circling the accelerator until the beam intensity becomes too low or the beam is no longer focused adequately.

A useful quantity to describe the performance of an accelerator is the factor of proportionality between the event rate $\mathrm{d} \mathcal{N} / \mathrm{d} t$ and the interaction cross section $\sigma(s)$, the so-called luminosity $\mathcal{L}$ :

$$
\mathrm{d} \mathcal{N} / \mathrm{d} t=\mathcal{L} \cdot \sigma(s)
$$

For the case of a p $\bar{p}$ collider, with $n$ bunches per direction and $N_{p}$ and $N_{\bar{p}}$ protons and anti-protons per bunch respectively, circulating with frequency $f$, the luminosity is given by

$$
\mathcal{L}=f \frac{n N_{p} N_{\bar{p}}}{4 \pi \sigma_{x} \sigma_{y}},
$$

where $\sigma_{x}$ and $\sigma_{y}$ characterise the Gaussian transverse beam profiles in the horizontal and vertical directions ${ }^{1}$. The luminosity lifetime of the beam at the Tevatron is approximately 9-10 hours if measured from the exponential decline in luminosity over the first two hours after injection. The real lifetime can be slightly higher as some mechanisms causing beam losses become weaker at lower luminosity.

In March 2001 the Tevatron entered its Run II. Within a few years of running it will provide a time-integrated luminosity of $2 \mathrm{fb}^{-1}$ at $\sqrt{\mathrm{s}}=2 \mathrm{TeV}$. The instantaneous luminosity is expected to reach up to $2 \times 10^{32} \mathrm{~cm}^{-2} \mathrm{~s}^{-1}$.

\footnotetext{
${ }^{1}$ The beam profiles $\sigma_{x}$ and $\sigma_{y}$ are taken to be equal for the proton and the anti-proton beam.
} 


\subsection{The Collider Detector at Fermilab}

CDF is a 5000 ton detector dedicated to the study of interactions occurring in the collisions of protons and anti-protons at the Tevatron. The upgrade of the accelerator complex also required a major upgrade of the CDF detector itself. This was motivated by the increase in instantaneous luminosity and the shorter bunch crossing times rather than the slight increase in energy. The bunch crossing time sets a time constant for signal integration, triggering and data acquisition. At present the time between bunches is $396 \mathrm{~ns}$; however in the future it is hoped that the number of bunches will be increased to 108 resulting in the shorter bunch crossing time of $132 \mathrm{~ns}$. This may well not be achieved, but these short times are the reason for much of the CDF upgrade.

Another input driving the new design is the average number of hadronic interactions $\overline{\mathcal{N}}$ in a single bunch crossing. This number depends on the luminosity and the bunch setup at CDF. Figure 3.4 depicts $\overline{\mathcal{N}}$ for different setups at CDF.

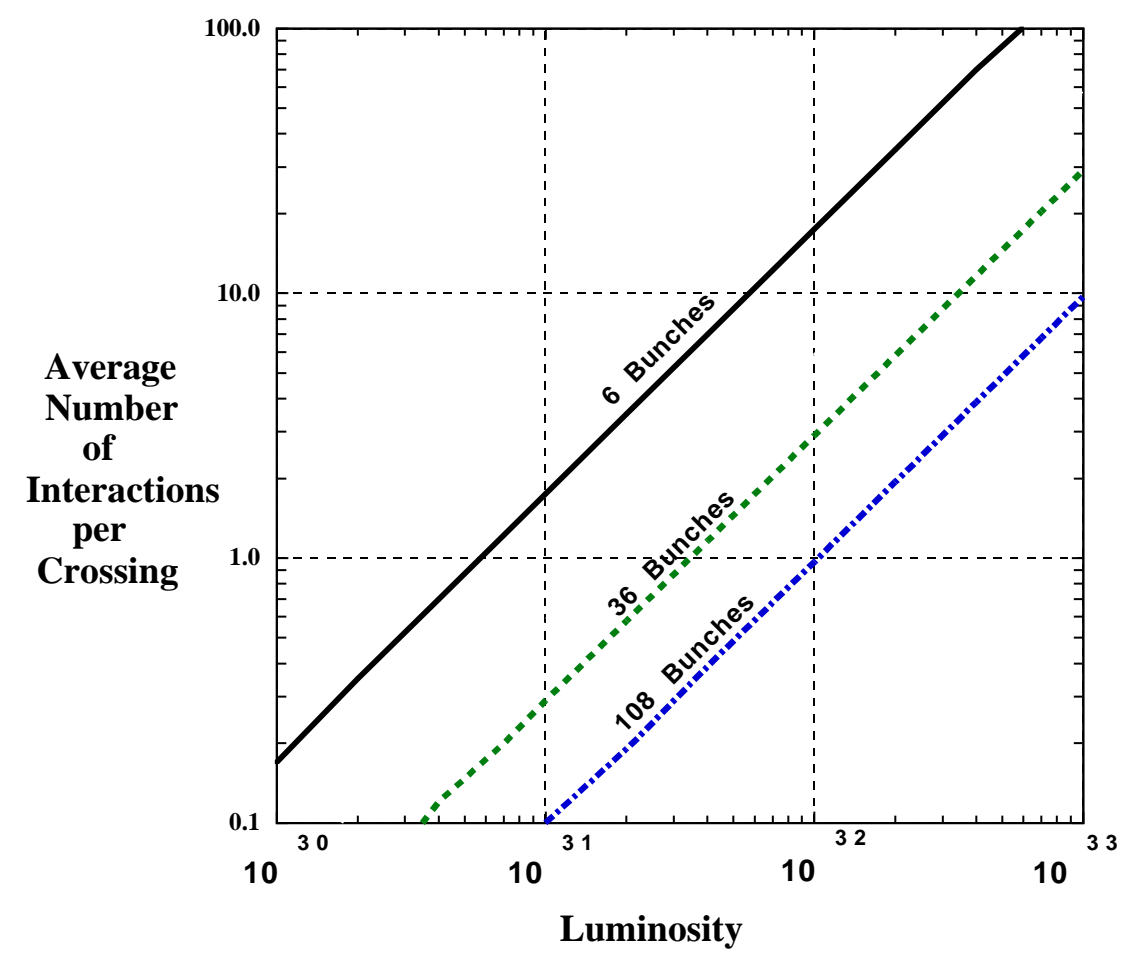

Figure 3.4: $\overline{\mathcal{N}}$ for various conditions at CDF. 36 bunches $\equiv 396 \mathrm{~ns}$ bunch crossing time, 108 bunches $\equiv 132$ ns bunch crossing time and 6 bunches $\equiv$ 3500 ns bunch crossing time (=Run I setup). 
For the two running conditions discussed for Run II, $\overline{\mathcal{N}}$ is obtained to be

- $\overline{\mathcal{N}} \sim 3$ for $\mathcal{L}=1 \times 10^{32} \mathrm{~cm}^{-2} \mathrm{~s}^{-1}$ and $396 \mathrm{nsec}$

- $\overline{\mathcal{N}} \sim 2$ for $\mathcal{L}=2 \times 10^{32} \mathrm{~cm}^{-2} \mathrm{~s}^{-1}$ and $132 \mathrm{nsec}$

and the detector is designed to operate under both conditions. Obviously the design is also influenced in a major way by the type of physics processes the accelerator can provide and the collaborators wish to study. At the Tevatron a multifold of different physics processes can be produced in an interaction, resulting in a very rich physics program ranging from top and b-physics to CP-violation and searches for new particles such as Higgs bosons and supersymmetric particles. CDF is thus designed as a general purpose collider detector which combines high precision tracking with fast projective calorimetry and fine grained muon detection with as large a spatial coverage as possible.

Figure 3.5 shows a solid cutaway view of the upgraded CDF detector (CDF II).

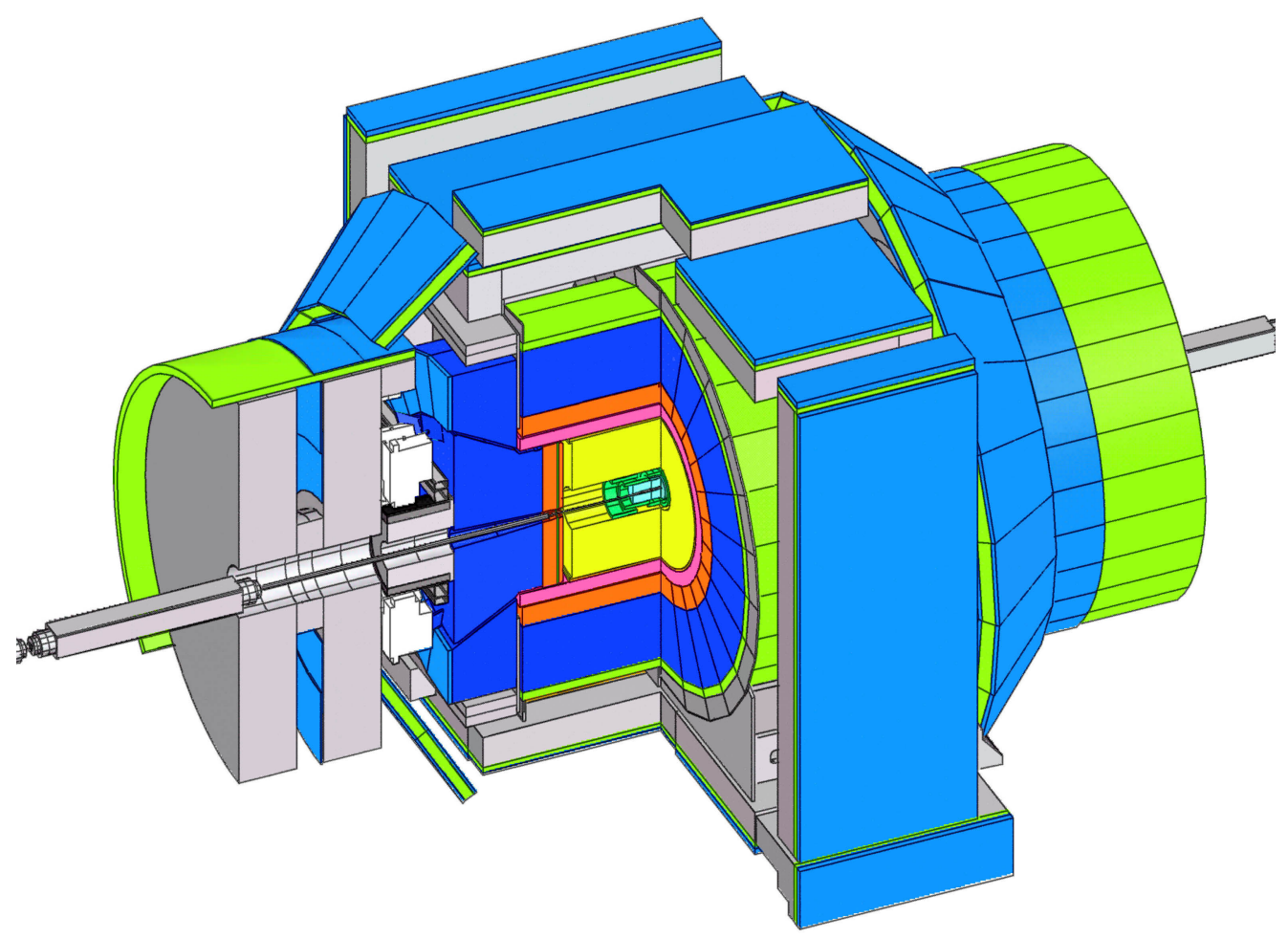

Figure 3.5: Solid cutaway view of the CDF II detector. 


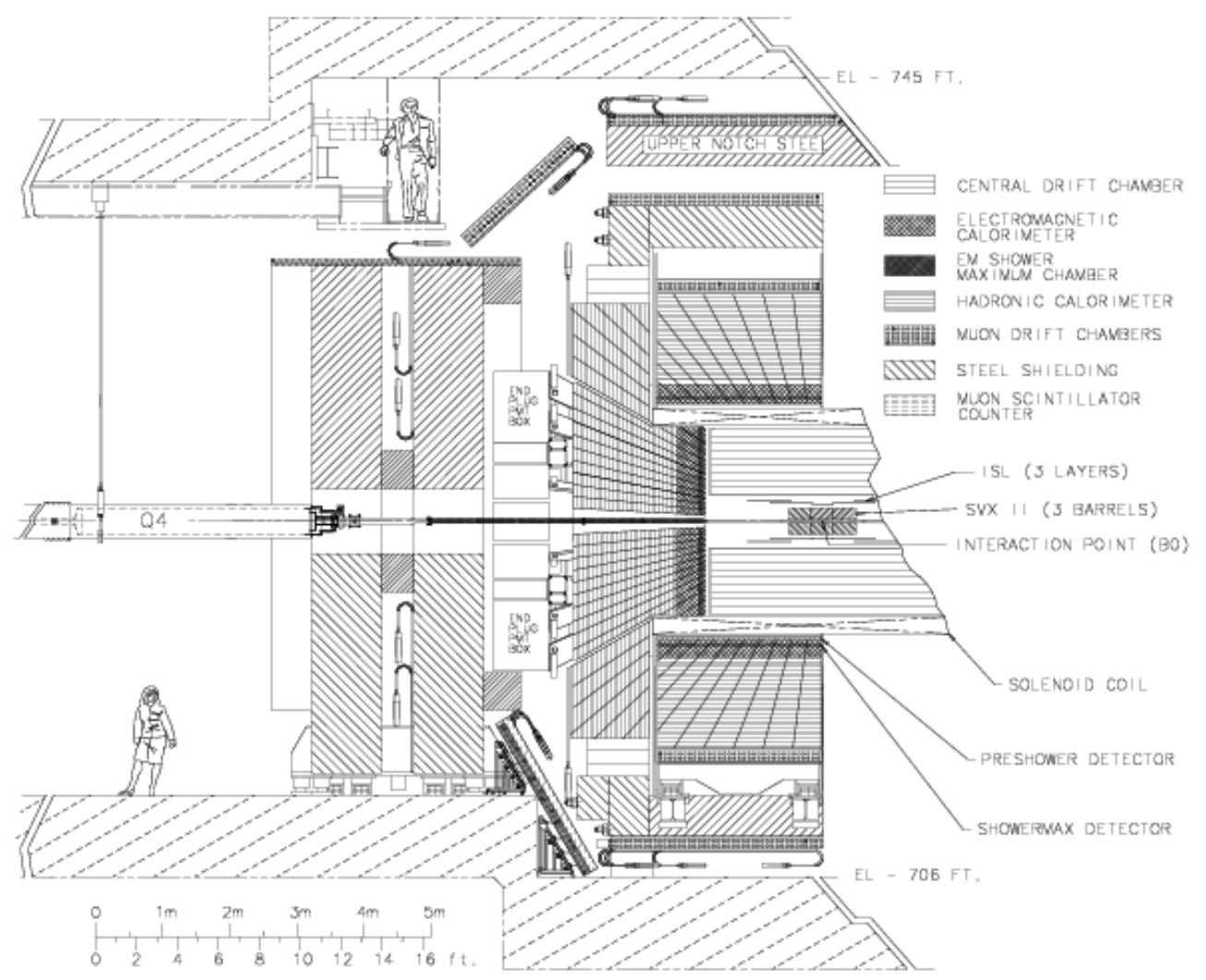

Figure 3.6: Elevation view of one half of the CDF II detector.

A cross section of one half of the detector is depicted in figure 3.6.

Two sub-detector systems, the time-of-flight detector and an additional silicon detector directly surrounding the beam pipe, are absent in this figure. They were proposed after the publication of the Technical Design Report (TDR, [25]). More information on these subsystems can be found in [26].

The coordinate system of CDF is as follows. The $x$-axis lies in the Tevatron plane and points away from the centre of the accelerator. The $y$-axis is perpendicular to the $x$-axis and points upwards. The $z$-axis points along the proton direction. The polar angle $\Theta$ is measured from the positive $z$-axis and the azimuthal angle $\phi$ is measured from the $x$-axis. The rapidity $y$ is defined as

$$
y=\frac{1}{2} \ln \left(\frac{E+p_{z}}{E-p_{z}}\right) .
$$

$E$ and $p_{z}$ are the particle's energy and longitudinal momentum respectively. For ultra-relativistic particles this quantity becomes approximately equal to the pseudo- 
rapidity $\eta$ :

$$
\eta=-\ln \left[\tan \left(\frac{\Theta}{2}\right)\right] .
$$

This quantity is frequently used at hadron collider experiments. The benefit of the rapidity (and the pseudo-rapidity if the mass of the particles can be neglected) is that a boost $\beta$ along the beam axis will shift the $y$ value of all particles in an event by

the same amount, $\tanh ^{-1} \beta$, when viewed in the laboratory frame, thus leaving the shape of the $y$ distribution as well as differences in $y$ between particles unchanged.

In the following the sub-detectors are briefly described in the order a particle generated at the primary interaction point would encounter them, i.e. from small to large radii with respect to the beam line. Emphasis lies on detectors most relevant to this analysis, i.e. on detectors in the central region of the detector.

\subsubsection{Tracking Detectors}

The efficient and precise determination of charged particle tracks is of the utmost importance to the CDF physics program. The tracking devices form the innermost part of the detector. They are located within a solenoid of $1.5 \mathrm{~m}$ radius and $4.8 \mathrm{~m}$ length which provides a magnetic field of approximately $1.4 \mathrm{~T}$ to bend the path of charged particles for momentum measurements.

The tracking system is composed of silicon devices, which CDF has established to be viable in a hadron collider environment, and a large open cell drift chamber consisting of 2520 drift cells. All of these sub-detectors were built for Run II to extend the tracking coverage up to $|\eta|<2$ and to account for the higher luminosity and shorter bunch crossing times.

\section{The Silicon System}

The silicon system comprises three concentric sub-detectors covering a region of $|\eta|<2$ and radii of $1.35 \mathrm{~cm}$ to $29 \mathrm{~cm}$ from the beamline. Figure 3.7 shows a cross section of this system.

A charged particle produced at the primary interaction point will first leave a signal in the innermost silicon layer called Layer 00 (L00) which directly surrounds 


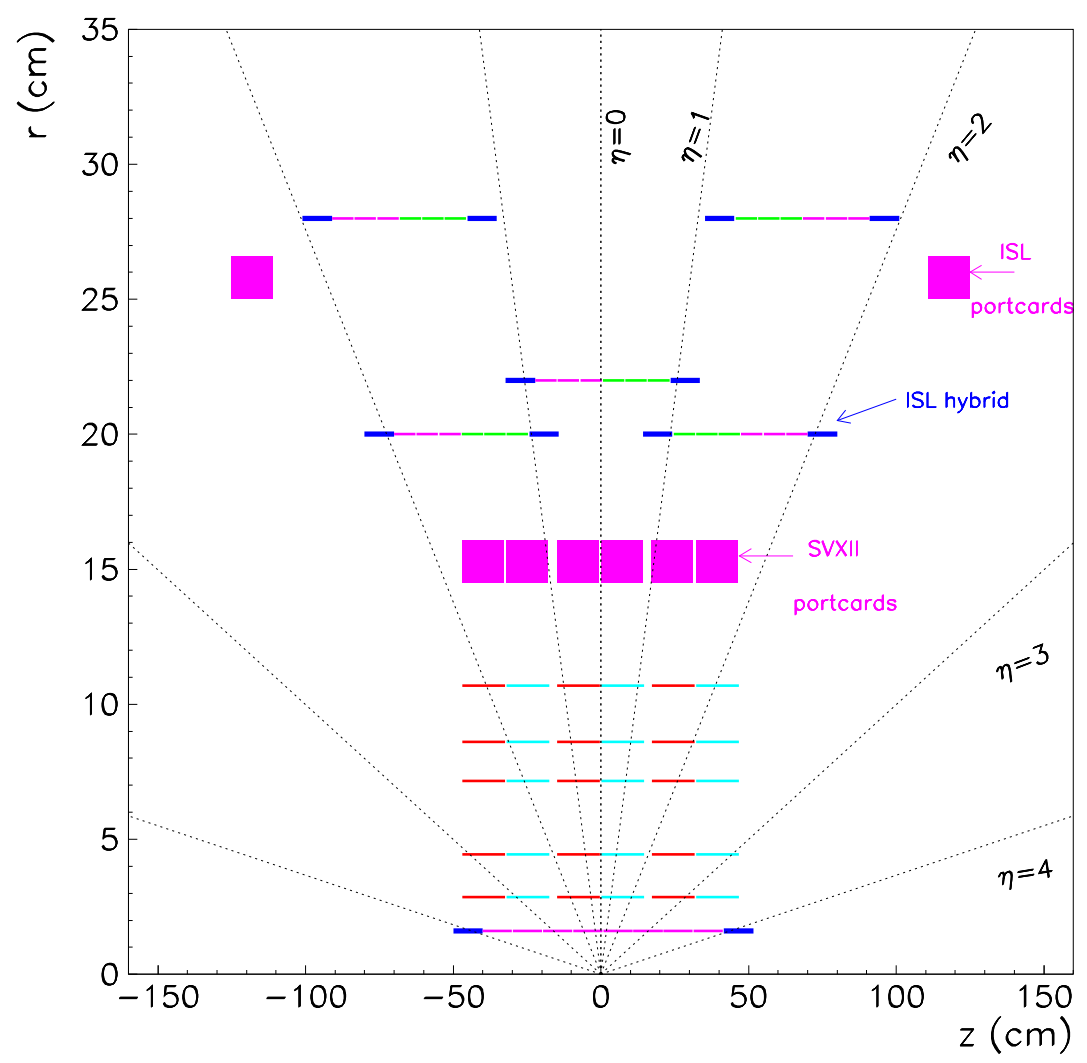

Figure 3.7: Schematic layout of the silicon tracking system. The innermost layer, Layer 00 consists of 6 sensors in $\mathrm{z}$.

the beam-pipe. This most recent addition to the tracking system is designed to improve the impact parameter resolution as well as the b-tagging efficiency. Benefiting from the advent of radiation tolerant silicon developed for the LHC at CERN this innermost layer consists of single sided radiation hard $\mathrm{p}$ on $\mathrm{n}$ silicon and will enable CDF to maintain high precision tracking and triggering beyond the lifetime of the adjacent silicon layer. It is expected that this layer, which was the innermost layer in the baseline design of CDF II, will become inoperable after an integrated luminosity of $5 \mathrm{fb}^{-1}$ is reached. L00 is divided into 12 wedges of sensors in $\phi$ as depicted in figure 3.8 (a), which are physically mounted on and supported by the beam pipe.

Six of the 12 sensors have a width of $8.4 \mathrm{~mm}$ with 128 readout channels; the remaining six have a width of $14.6 \mathrm{~mm}$ with 256 readout channels. They are mounted at radii $\mathrm{r}=1.35$ and $\mathrm{r}=1.62$ respectively. In the $z$ direction each sensor in $\phi$ comprises 6 individual sensors mounted end to end. Kapton cables carry the signal from the 


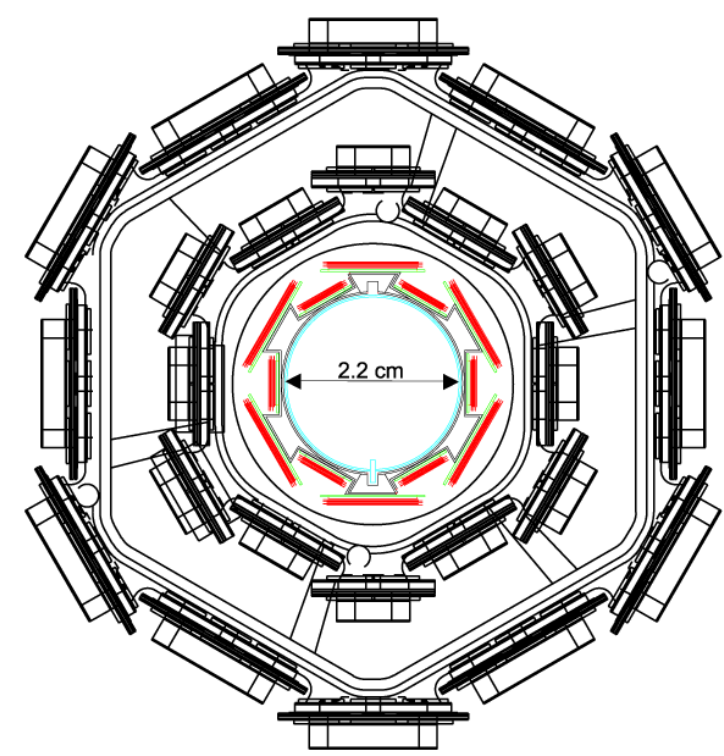

(a)

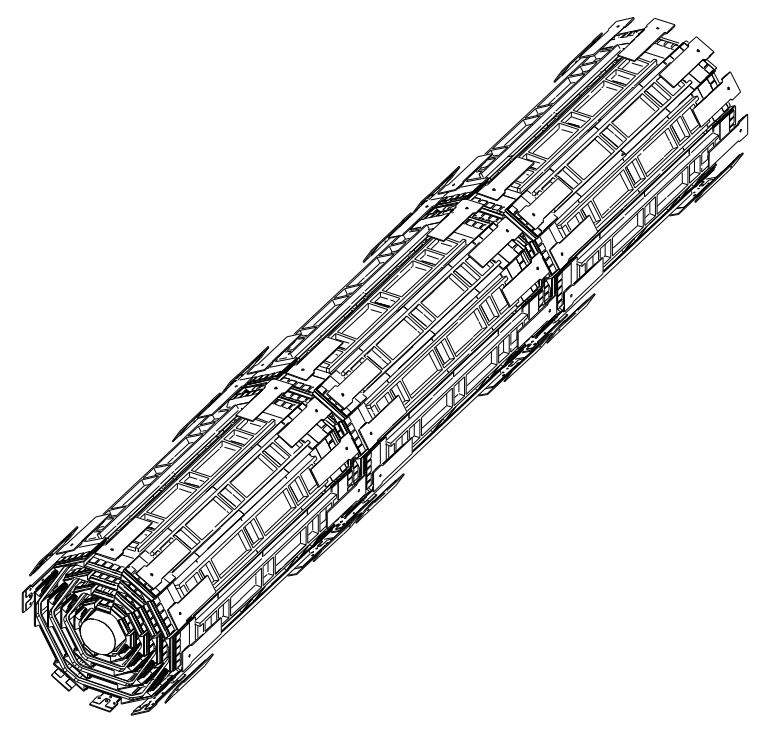

(b)

Figure 3.8: Figure (a) shows the $\mathrm{r}-\phi$ view of L00 (innermost cylinder cross section) and the first two layers of the SVX II detector. Figure (b) shows a perspective view of the three barrels of the SVX II detector.

six pairs of low and high width sensors to the readout electronics mounted at the ends of the array, outside the sensitive volume.

The next system is the Silicon VerteX detector SVX II, an integral part of the CDF upgrade for Run II. It comprises three cylindrical barrels lined in the $z$ direction, each of which has an active length of $29 \mathrm{~cm}$. Each barrel consists of 12 wedges in $\phi$ with five layers of double sided AC coupled silicon, spanning a range in radii from $2.5 \mathrm{~cm}$ for the first layer (layer 0) to $10.6 \mathrm{~cm}$ for the fifth layer (layer 4 ). The $\mathrm{r}-\phi$ view of the first two layers is shown in figure 3.8 (a). One side of each layer provides an $\mathrm{r}-\phi$ measurement, while the strips on the other side are oriented at $90^{\circ}$ with respect to the $z$-axis for layers 0,1 and 3 (referred to as $\mathrm{r}$ - $z$ measurement) and at $1.2^{\circ}$ for layers 2 and 4 (referred to as small angle stereo measurement or SAS) thus enabling three dimensional reconstruction of secondary vertices of long lived particles. The structure of SVX II with its three barrels is depicted in figure 3.8 (b).

The beryllium bulkheads on which the ladders of the three barrels are mounted 
carry the water cooling channels for the readout electronics, custom-designed radiation hard SVX3D readout chips [27].

Altogether the SVX II has just over 400000 readout channels compared to 46080 for the SVX detector in Run I.

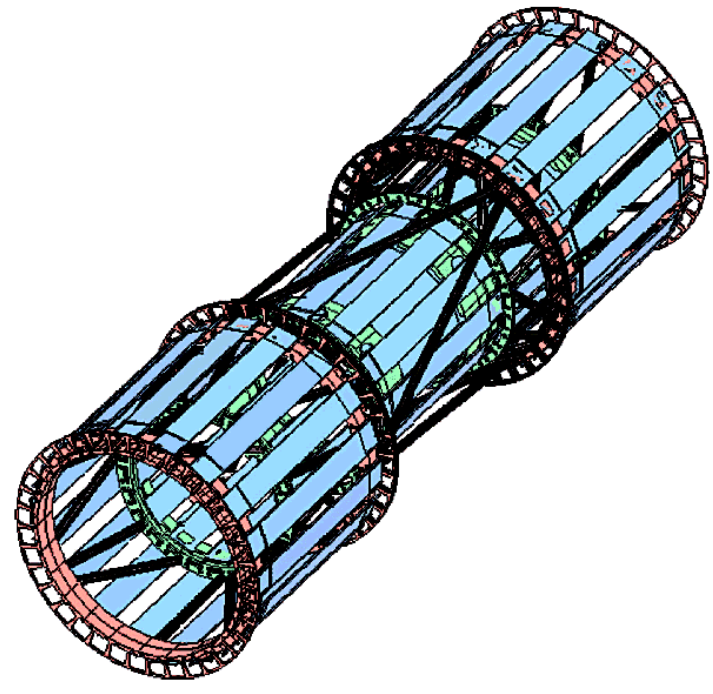

(a)

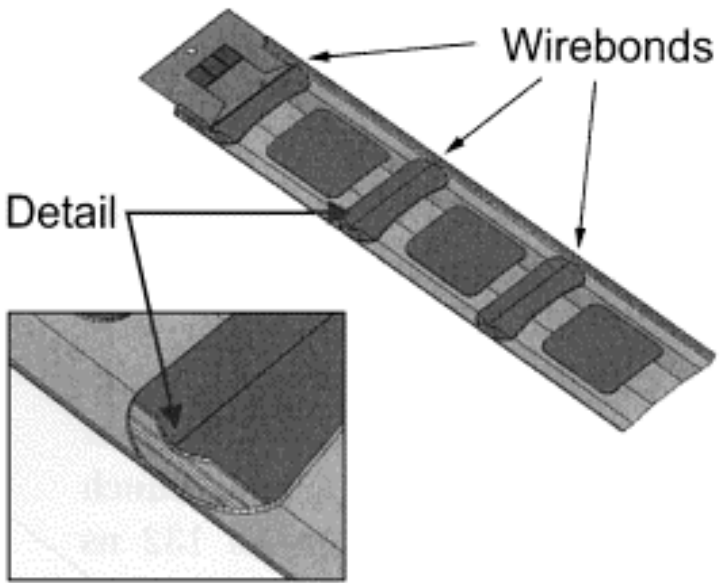

(b)

Figure 3.9: Figure (a) shows a perspective view of the ISL space frame and the silicon ladders. For better perspective view only every other silicon ladder is shown. Figure (b) [28] shows the design of a half ladder of the ISL detector.

The outermost silicon detector is called ISL for Intermediate Silicon Layer. It closes the spatial gap between SVX II and the central drift chamber and aids in combining the information from the two. It consists of a single layer of silicon in the central region $|\eta|<1.1$ at a radius of $22 \mathrm{~cm}$ and two layers at either side of the central layer at $r=20 \mathrm{~cm}$ and $r=28 \mathrm{~cm}$ covering the rapidity region $1 \leq|\eta| \leq 2$. These two layers in the side region are motivated by the desire to have 3D tracking available for $1 \leq|\eta| \leq 2$, a region where SVX II on its own can only provide $2 \mathrm{D}$ tracking. The silicon sensors consist again of double sided silicon crystals. One side provides $\mathrm{r}-\phi$ measurements via axial microstrips, the other side supplies $z$ information via stereo strips (placed at $1.2^{\circ}$ for all layers). Each half ladder is made from three crystals which are microbonded to each other end to end. The ladders are supported by a carbon fibre space frame with beryllium cooling 
ledges. The half ladders are combined into a full ladder with readout modules at either end of this ladder. The readout hybrids are mounted off the silicon and glued onto the edge of the carbon fibre support. Figure 3.9 (a) shows a model of the ISL detector itself and figure 3.9 (b) shows one of its half ladders.

\section{The Central Drift Chamber}

The Central Outer Tracker (COT) is a large cylindrical open cell drift chamber. It was designed to replace the old Central Tracking Chamber (CTC) of Run I. With a maximum drift time of $706 \mathrm{~ns}$ compared to bunch crossing times of $396 \mathrm{~ns}$ and $132 \mathrm{~ns}$, the CTC would have suffered from severe occupancy problems. Shorter drift times can be achieved by choosing a gas with higher drift velocity and by minimising the cell size and thus the drift distance. An argon/ethane $/ \mathrm{CF}_{4}$ mixture of 50:35:15 is found to have a drift velocity of $88 \mu \mathrm{m} / \mathrm{ns}$. Reducing the size of the drift cells by about a factor of four compared to the CTC results in a maximum drift distance of $0.88 \mathrm{~cm}$ which in conjunction with the faster drift velocity yields a maximum drift time of $100 \mathrm{~ns}$, short enough for operation at $132 \mathrm{~ns}$ bunch crossing times. For the 396 ns bunch crossing time it is sufficient to use a 50:50 argon/ethane mixture. To reduce the ageing rate, the gas is bubbled through isopropyl alcohol which also allows the COT to be operated at a higher voltage. The chamber covers the rapidity range $-1<|\eta|<1$ with a length of $310 \mathrm{~cm}$ of active volume. It covers the full $360^{\circ}$ in $\phi$. The radial region from $r=43.4 \mathrm{~cm}$ to $r=132.3 \mathrm{~cm}$ is covered by 96 sense wires which are grouped into eight "superlayers" as indicated in figure 3.10.

Each superlayer is divided into a set of drift cells arranged concentrically around the beamline. Each drift cell contains 13 potential wires interspaced by the 12 sense wires. All wires are gold plated tungsten with a diameter of $40 \mu \mathrm{m}$. The wire planes are sandwiched between gold coated $6.35 \mu \mathrm{m}$ thick Mylar planes which complete the field region. Each plane is shared by adjacent cells. Stainless steel wires are epoxied along the edges. The wires are attached in a parabolic shape to keep the sheets flat under tension. The ends of the cells are closed electrostatically and mechanically by Mylar strips with field shaping wires attached to them. Figure 3.11 shows three drift cells. To ensure short drift distances the cells are tilted by $35^{\circ}$, which is the Lorentz angle in the COT. Axial superlayers alternate with stereo superlayers (the wires are 


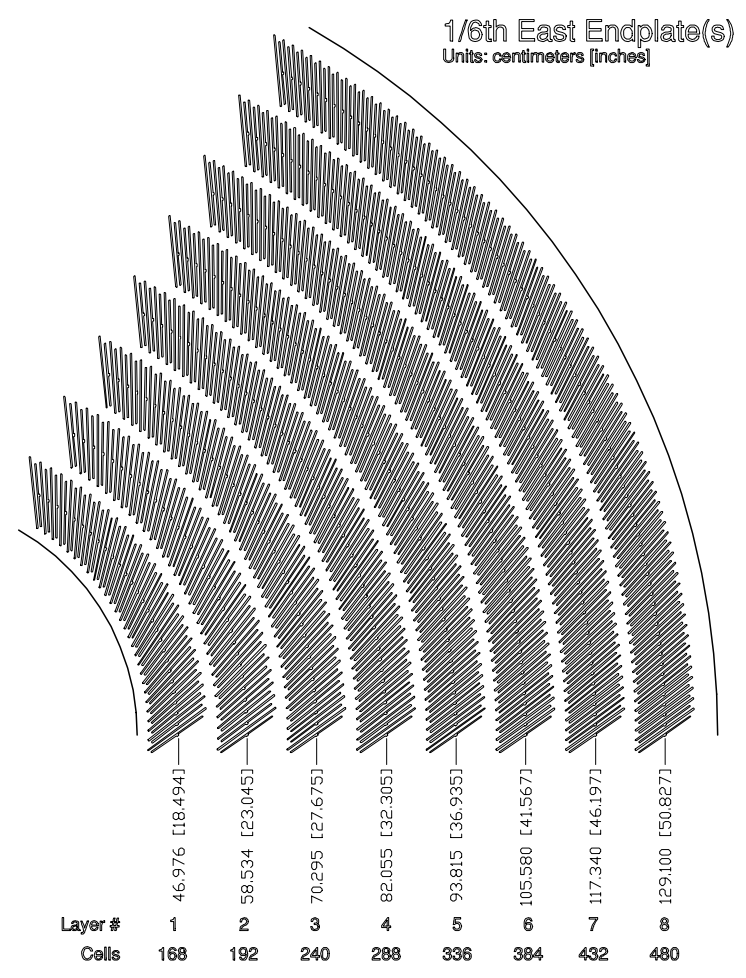

Figure 3.10: East endplate of the COT. Visible are the slots that hold the wires with subdivision into eight superlayers.

here tilted at $\pm 2^{\circ}$ with respect to the axial direction) to provide $\mathrm{r}-\phi$ as well as $\mathrm{r}-z$ measurements. In total the chamber consists of 30240 sense wires and the charge collected at these wires can be turned into information about the energy loss per unit length and thus provide important means of particle identification. Readout electronics as well as high voltage distribution are mounted on the chamber face. On one side high voltage motherboards plug into pairs of wire planes. Motherboards with chips for amplification, signal shaping and discrimination are connected to pairs of wire planes on the other end. They are then connected to pipelined time to digital converters (TDCs) from which the chamber signals are read out providing information available at the first trigger level.

The transverse momentum resolution achieved with the COT is measured - using cosmic ray events [29] - to be:

$$
\sigma\left(\mathrm{p}_{\mathrm{T}}\right) / \mathrm{p}_{\mathrm{T}}^{2}=0.0017(\mathrm{GeV} / \mathrm{c})^{-1} .
$$

Table 3.1 details the single hit resolutions obtained with the various components of the CDF tracking system. 


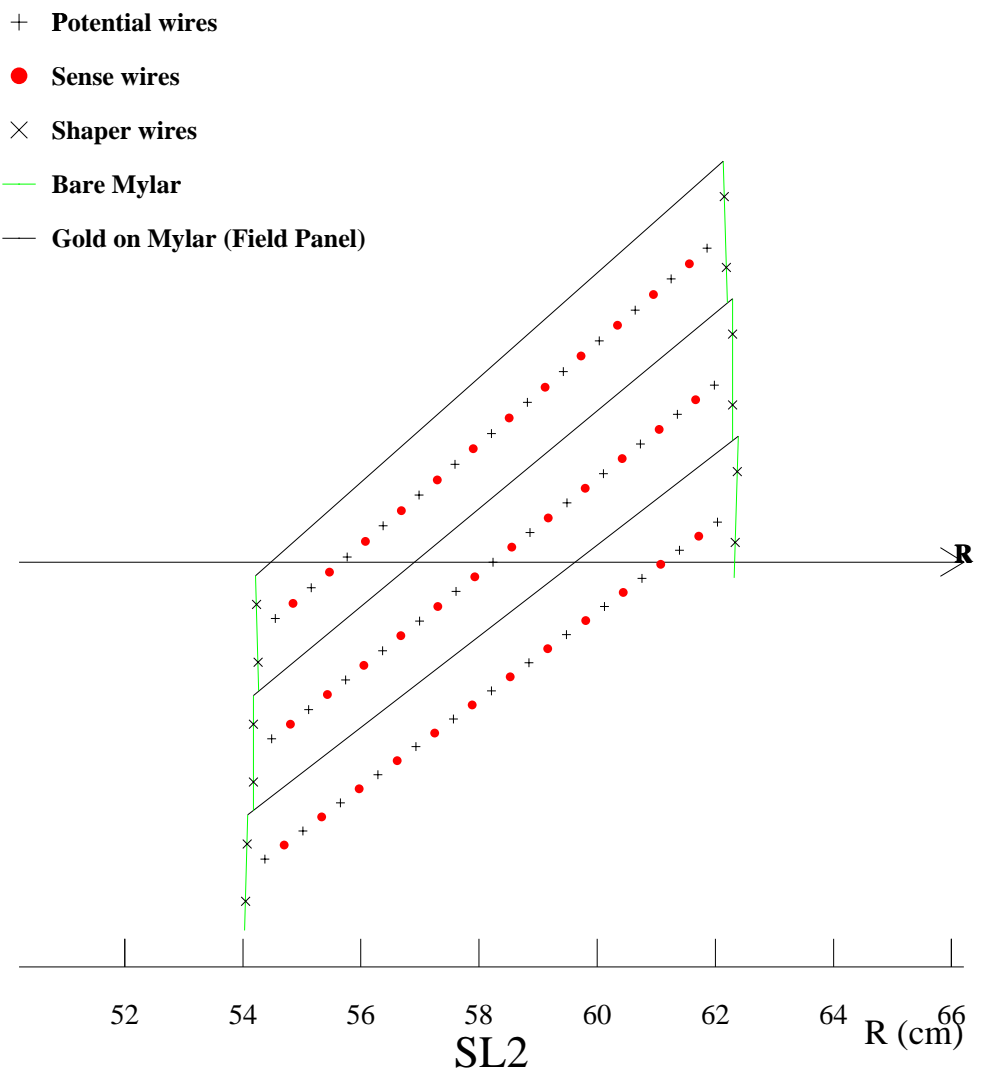

Figure 3.11: Three drift cells of the COT in superlayer 2, looking along the $z$ direction.

Using the hit information from the SVX II detector, an average impact parameter resolution $\sigma\left(d_{0}\right)$ of $34 \mu \mathrm{m}$ is achieved for muon tracks with a transverse momentum of approximately $1.5 \mathrm{GeV} / \mathrm{c}$ [29]. After inclusion of the uncertainty on the transverse size of the beamspot, this resolution increases to approximately $50 \mu \mathrm{m}$.

\begin{tabular}{|c|c|c|}
\hline & Coverage & Position Resolution \\
\hline L00 & $|\eta|<4$ & $6 \mu \mathrm{m}$ (axial) \\
\hline SVX II & $|\eta|<2$ & $12 \mu \mathrm{m}$ (axial) \\
\hline ISL & $|\eta|<2$ & $16 \mu \mathrm{m}$ (axial) \\
\hline COT & $|\eta|<1$ & $180 \mu \mathrm{m}$ \\
\hline
\end{tabular}

Table 3.1: Summary table of the position resolution of the various components of the CDF tracking system [30]. 


\subsubsection{The Time-Of-Flight Detector}

The Time-Of-Flight detector (TOF) is a system of scintillators which was added to the baseline design of the CDF II detector to enhance particle identification for momenta below $2 \mathrm{GeV} / \mathrm{c}$ by precisely measuring the time a particle needs to travel from the interaction point to the TOF system. It is fitted into the radial space between the COT and the cryostat for the superconducting solenoid at $r=138 \mathrm{~cm}$ as shown in figure 3.12 (a).

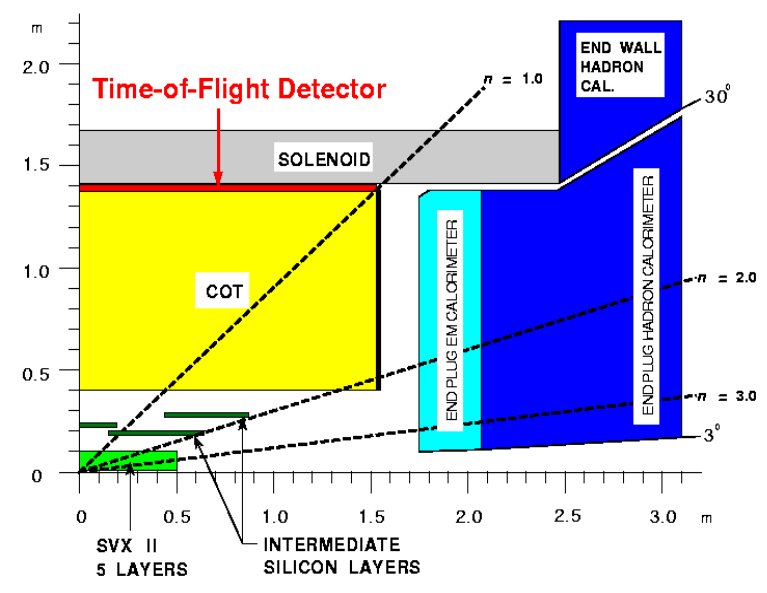

(a)

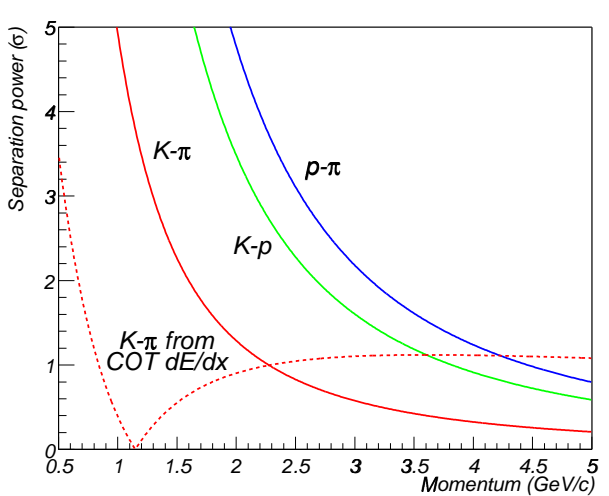

(b)

Figure 3.12: Figure (a) shows the location of the TOF detector and the new calorimeter system in the plug region within the CDF II detector. Figure (b) shows the separation power obtained from the time difference as a function of momentum between $\mathrm{K} / \pi, \mathrm{p} / \mathrm{K}$ and $\mathrm{p} / \pi$ assuming a timing resolution of 100 ps. The $\mathrm{K} / \pi$ separation obtained using $\mathrm{dE} / \mathrm{dx}$ values from the COT is shown for comparison.

The main physics motivation being $\pi / K$ identification for $\mathrm{b}$ flavour tagging it is also used for cosmic ray rejection and signal over background enhancement in hadron reconstruction. The TOF detector is built of 216 bars of Bicron BC-408 scintillator with dimensions $4 \mathrm{~cm} \times 4 \mathrm{~cm} \times 280 \mathrm{~cm}$. The radial thickness was determined by the space available and the azimuthal width was determined by occupancy and resolution considerations. The bars are trapezoidal in shape to maximise the instrumented regions. They cover $|\eta|<1.1$.

Each end of the scintillators is equipped with a photomultiplier with a diameter of 1.5 inch (Hamamatsu R7761, 19 stage, fine-mesh [31]). Their usual gain of about 
$10^{6}$ is reduced to about $3 \times 10^{4}$ inside the $1.4 \mathrm{~T}$ magnetic field. The contribution of the electronics to the timing resolution is measured to be smaller than 25 ps [32].

The photomultipliers yield time and pulse-height information. The time information together with the time of the beam crossing is used for particle identification. With the momentum $p$ and the pathlength $L$, as measured with the tracking system, the time $t$ can be used to calculate the mass $m$ of a particle and thus to determine the particle type using:

$$
m=\frac{p}{c} \sqrt{\frac{c^{2} \cdot t^{2}}{L^{2}}-1} .
$$

Figure 3.12 (b) [32] shows the separation power that would be achieved assuming a timing resolution of $100 \mathrm{ps}$. The separation from $\mathrm{dE} / \mathrm{dx}$ measurements with the COT is shown for comparison for $\mathrm{K} / \pi$. The current mean time resolution measured from all channels is $122 \mathrm{ps}$ with an rms spread of $20 \mathrm{ps}$ [32]. Comparing readings at either end yields a $z$ measurement which is used to assign a TOF signal to a track reconstructed with the tracking detectors.

\subsubsection{The Calorimeter System}

While tracking detectors are low density position measurement devices, calorimeters are total absorption detectors designed to determine the energy of particles.

Electromagnetic calorimeters are built to provide an energy measurement for electrons (positrons) and photons ${ }^{2}$. For energies above a few $100 \mathrm{MeV}$, the main processes that occur when these particles enter the material are bremsstrahlung for electrons and pair production for photons. These two processes lead to the development of an electromagnetic shower, which is depicted in the left part of figure 3.13; a photon enters the detector material and produces an electron-positron pair with its energy distributed between the two particles. After traversing a radiation length $X_{0}$ of material the probability for the photon to produce such a pair is $54 \%$. The newly created particles then radiate new photons in the field of nuclei, a process known as bremsstrahlung. The shower ends when the energy of the particles falls below the

\footnotetext{
${ }^{2}$ Electrons and positrons exhibit the same behaviour and in the following positrons are implicitly included in the description when electrons are mentioned.
} 
critical energy necessary to create new electron-positron pairs. At this point ionisation and excitation are the dominant processes. The length of the electromagnetic calorimeter is designed such that electrons and photons dissipate all their energy in the detector volume.

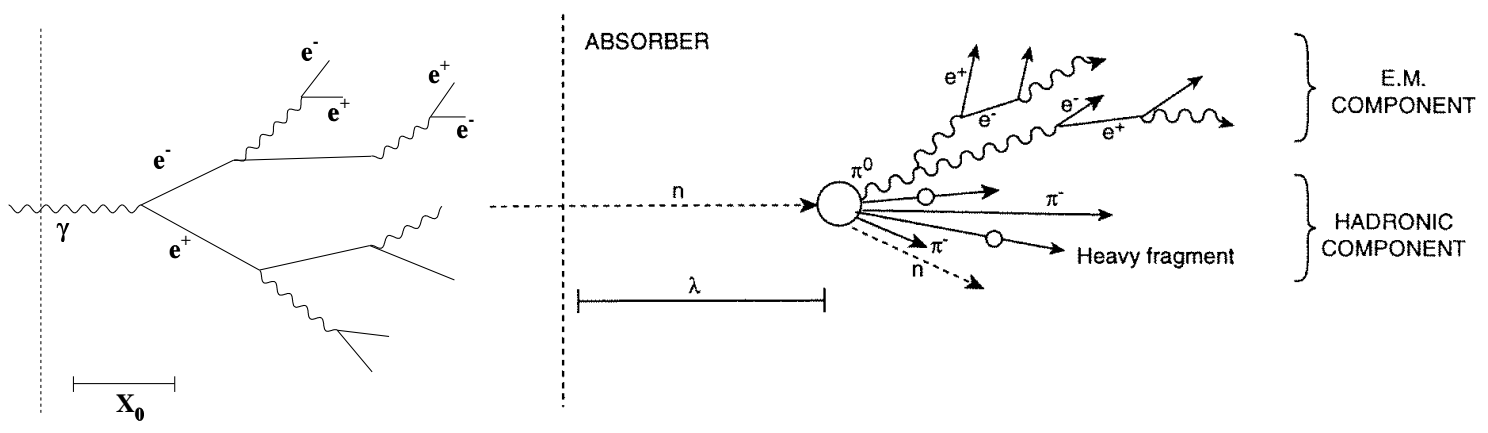

Figure 3.13: Development of an electromagnetic shower (left) and a hadronic shower (right [33]).

\section{CDF's Central Calorimeter}

For this work only the central calorimeter is used. It extends to $|\eta|<1.1$ and is, apart from the electronics, largely unchanged from the Run I calorimeter.

\section{Central Pre Radiator}

Even though the tracking detectors are designed to contain as little material as possible, the magnet coil and the cryostat of the superconducting solenoid themselves comprise already 0.86 to 1.1 radiation length of material [34] depending on their angle with respect to the $z$-axis.

This material acts as absorber, or "radiator" and forms, in conjunction with a single Multi Wire Proportional Chamber (MWPC) plane, the innermost part of CDF's calorimeter system, the Central Pre Radiator (CPR). The MWPCs are fitted into a $5 \mathrm{~cm}$ gap between the solenoid and the central electromagnetic calorimeter. Altogether there are 96 chambers; each chamber contains 32 sense wires and 31 field wires and is filled with non-flammable $\mathrm{Ar} / \mathrm{CO}_{2}$. The system is installed at a distance of $168.29 \mathrm{~cm}$ from the beampipe to sample showers that are initiated before the actual electromagnetic calorimeter. The passive material in conjunction 
with the MWPCs provides an excellent way of aiding in the measurement of prompt gamma production and electron pion separation.

\section{Central Electromagnetic Calorimeter}

The Central Electro Magnetic calorimeter (CEM) follows radially outwards from the CPR. CDF's electromagnetic and hadronic calorimeter have a common structure, they are both sampling calorimeters with alternating layers of scintillator and absorbing material. To combine the good energy resolution of a scintillator with good position measurement, the CEM is additionally built as a hybrid of a scintillator based sampling calorimeter for energy measurement and a 2D strip chamber for position determination and measurement of the longitudinal shower development. The energy resolution achieved with this design is measured to be $\sigma(E) / E=$ $13.5 \% / \sqrt{E_{T}}$ with $E_{T}=E \times \sin \theta$ in $\mathrm{GeV}$. The position resolution achieved is $2 \mathrm{~mm}$ at $50 \mathrm{GeV}$. Following an inner base plate of aluminium the calorimeter consists of alternating layers of $5 \mathrm{~mm}$ thick SCSN-38 polystyrene scintillator [35] (31 layers in total) and $3.2 \mathrm{~mm}$ thick metal (30 layers). The metal comprises mainly lead with small amounts of aluminium. However, to retain constant radiation length thickness it is in places substituted with acrylic. The sandwiched layers can be divided into two halves in $\eta$, each stretching for about $2.5 \mathrm{~m}$ along the $z$-axis and into modules of $15^{\circ}$ in $\phi$, with small cracks at the boundaries, resulting in 48 modules in total. Each module is subdivided into 10 units along $z$ with $\delta \eta \approx 0.11$. The $\eta$ - $\phi$ modules are aligned to point back to the centre of the detector. Together with the cryostat the system comprises approximately $19 X_{0}$ of material. Wavelength shifters installed at the $\phi$ surfaces guide the light produced in the scintillators to photomultiplier tubes. Figure 3.14 shows one of the 24 wedges in $\phi$ with its light gathering system.

Embedded in between the eighth layer of lead and the ninth scintillator layer, which corresponds to a depth of maximum average shower development, at approximately $6 X_{0}$ inside the CEM is the Central Electromagnetic Strip detector CES. Consisting of orthogonal strips and wires the CES is designed to determine the shower position as well as the transverse shower development. The chambers are operated with an $\mathrm{Ar} / \mathrm{CO}_{2}$ mixture. For details of wire material and bonding see [36]. 


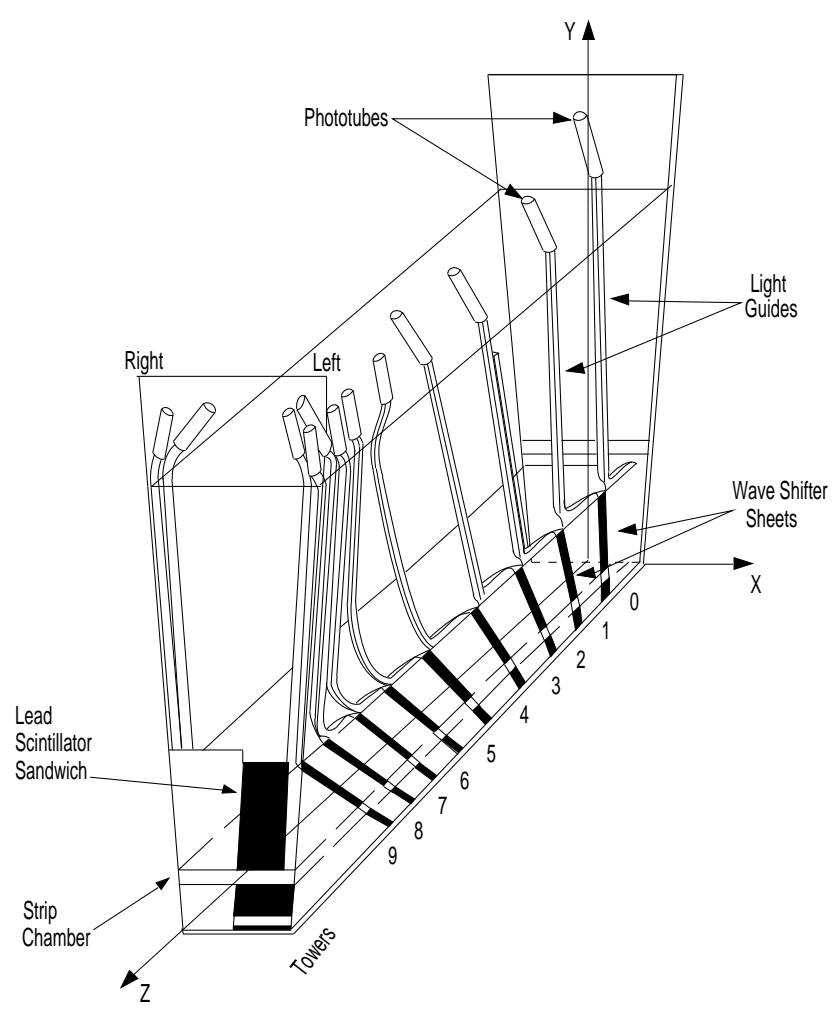

Figure 3.14: One wedge of the CEM with its light collecting system.

\section{Central Hadronic Calorimeter}

Hadrons have a different shower behaviour and the electromagnetic calorimeter does not have much stopping power for hadrons. Thus an additional calorimeter, the Central HAdronic calorimeter $(\mathrm{CHA})$ is built radially outside the electromagnetic calorimeter to measure the energy of hadrons. If hadrons of energies above about $5 \mathrm{GeV}$ impinge on a block of matter, inelastic as well as elastic scattering will occur between the particles and the nucleons inside the material. Thus energy is transferred and secondary hadrons are produced, that further collide inelastically. A hadron shower occurs. The basic interaction responsible for the shower development is the strong interaction. In addition, the shower has an electromagnetic component originating mainly from the decay of secondary $\pi^{0}$ 's, as can be seen in the right drawing of figure 3.13. A hadron calorimeter would equally initiate an electromagnetic shower and hadron calorimeters and electromagnetic calorimeters are built in much the same way. Electrons are however expected to be absorbed before they can reach the hadronic calorimeter, while the CEM only comprises one 
interaction length for pions. (A block of matter generally comprises fewer units of the characteristic variable for hadronic showers, the interaction length $\lambda$, than for the equivalent variable for electromagnetic showers, the radiation length $X_{0}$, which results in electromagnetic calorimeters being more compact than hadronic calorimeters).

The CHA, like the CEM, is a scintillator based sampling calorimeter. It extends from $-0.9 \leq \eta \leq 0.9$ and covers apart from the slightly smaller $\eta$ region essentially the region covered by the CEM with the towers of the CHA built in blocks of 0.11 $\times 15^{\circ}$ in the $\eta-\phi$ plane to match the towers of the CEM. The CHA also comprises 48 modules, each of which consists of 32 layers of $10 \mathrm{~mm}$ thick acrylic scintillator, PMMA (Poly Methyl MetAcrylate) napthalene, sandwiched between $2.5 \mathrm{~cm}$ thick iron absorber plates. The energy resolution of the CHA is $\sigma(E) / E=50 \% / \sqrt{E_{T}}$ with $E_{T}$ in $\mathrm{GeV}$ and it has a thickness of $4.5 \lambda$.

\section{The Plug Calorimeter}

Although not used for this analysis the plug calorimeter is briefly described. It was newly constructed for Run II to replace the existing gas calorimeter whose time response would have been incompatible with the bunch crossing times at Run II. It covers $1.1<\eta<3.6$. Figure 3.12 indicates the position of the new detector system and figure 3.15 gives a more detailed view of the upper part of the new end plug calorimeter. Like the central calorimeter it comprises an electromagnetic calorimeter EM, a shower maximum detector and a hadron calorimeter HAD. The active elements in both EM and HAD are scintillating tiles read out by wave length shifting fibres which in turn are spliced to clear fibres. The clear fibres are then connected to photomultiplier tubes. The scintillator is SCSN-38 (produced by the company Kuraray) which is chosen for its high light output and radiation resistance.

The electromagnetic calorimeter consists of 23 layers of $4 \mathrm{~mm}$ thick scintillator sandwiched between $4.5 \mathrm{~mm}$ thick layers of lead. The scintillator of the first layer is $10 \mathrm{~mm}$ thick and read out by multi-anode photo multiplier tubes thus acting as preshower detector. Altogether the EM has a total thickness of $35.7 \mathrm{~cm}$ or $23.2 X_{0}$ $(0.96 \lambda)$. The scintillator strips of the shower maximum detector are again placed at approximately $6 X_{0}$ into the detector. 


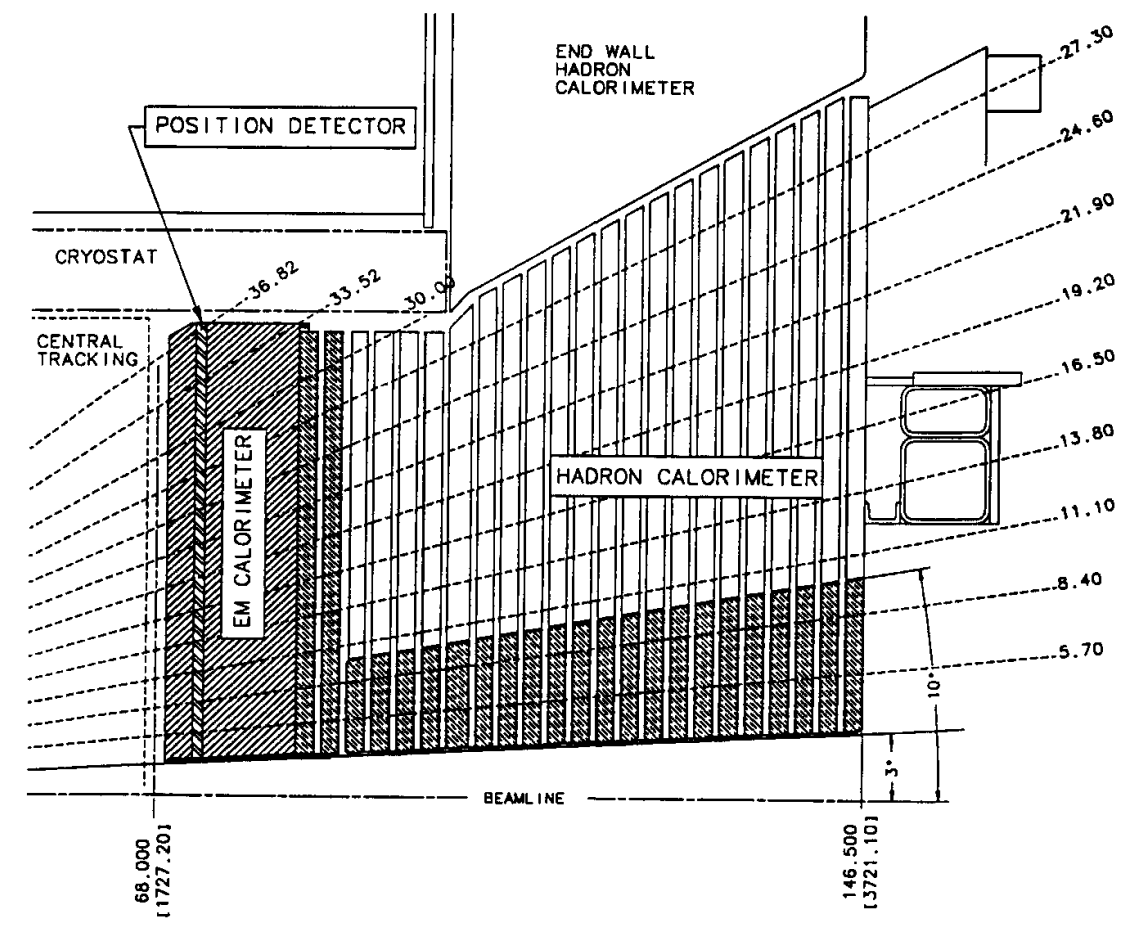

Figure 3.15: R- $z$ view of the top half of one plug of the new calorimeter for CDF II.

The hadron calorimeter consists of 23 layers of $6 \mathrm{~mm}$ thick scintillator placed between 2 inch thick layers of iron. It has a thickness of $160 \mathrm{~cm}$ or $6.8 \lambda$. The towers of EM and HAD point back to the centre of the detector and the subdivision in $\eta-\phi$ is similar to the one of the central calorimeter. More details can be found in the Technical Design Report [25]. The energy resolution of the end plug calorimeter is $\sigma(E) / E=14.5 \% / \sqrt{E(\mathrm{GeV})}$ plus a constant term of $0.7 \%$ for electrons and $\sigma(E) / E=$ $68.0 \% / \sqrt{E(\mathrm{GeV})}$ plus a constant term of $4.1 \%$ for charged pions [37].

\subsubsection{CDF's Muon System}

Four subsystems of muon chambers and scintillation counters form the outermost part of the CDF II detector. The calorimeter steel, the return yoke of the magnet, additional steel walls and steel from the Run I forward muon toroids provide sufficient absorber material to stop most hadrons, leaving only muons to reach these outermost chambers due to their higher penetration power. Occasionally a hadron 


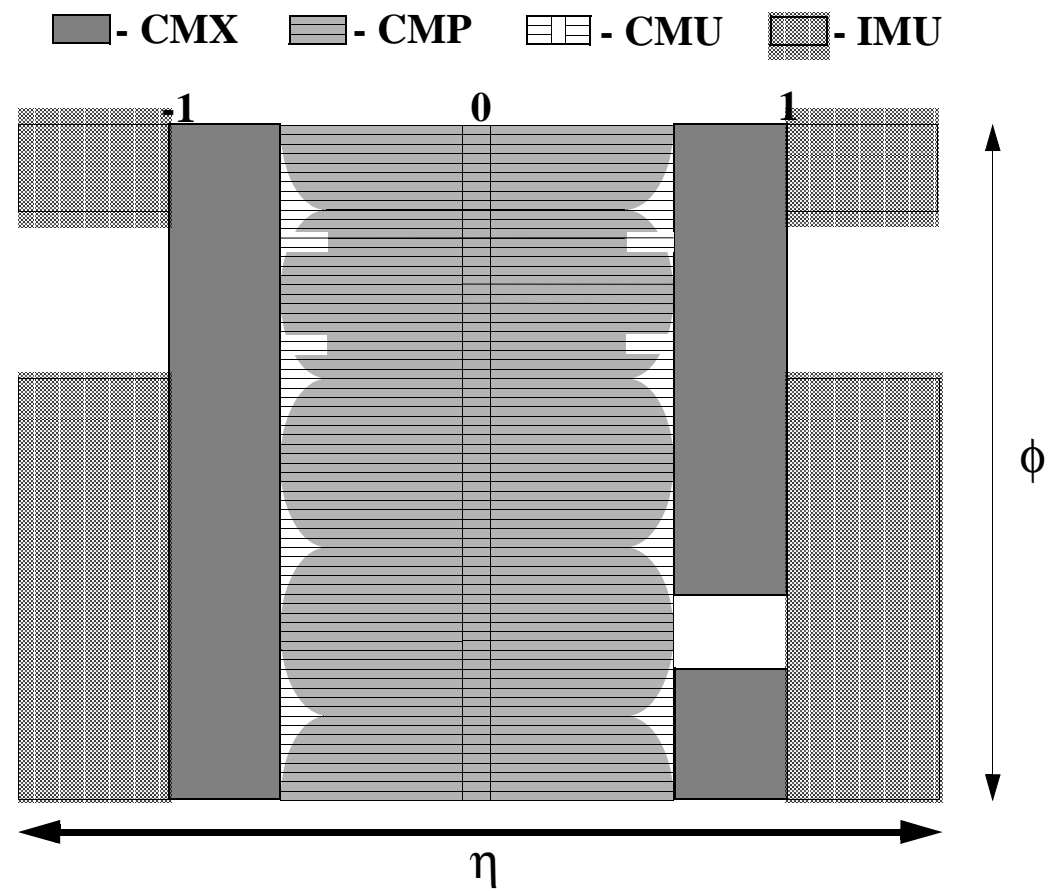

Figure 3.16: Location of the muon detectors for Run II in azimuth $\phi$ and pseudorapidity $\eta$.

can enter the muon system if it either fails to interact strongly in the absorber material or if the interaction occurred too close to the muon chambers allowing secondary particles to escape the calorimeter. This is called a punch-through. The subsystems of muon chambers, which are described in more detail below, cover together the region $|\eta| \leq 2$. Figure 3.16 shows their respective coverage in the $\eta$ - $\phi$ plane.

\section{The Central Muon Detector}

The Central MUon detector (CMU) is the oldest part of CDF's muon system and was only marginally changed for operation under Run II conditions. As indicated in figure 3.16 it covers $|\eta| \leq 0.6$ with a gap of $18 \mathrm{~cm}$ between the east and west chambers at $\eta=0$, equivalent to the range $55^{\circ} \leq \theta \leq 90^{\circ}$. The muon system is situated behind approximately $5.5 \lambda$ of absorber from the calorimeter which results in a $\mathrm{p}_{\mathrm{T}}$ threshold for muon detection of $1.4 \mathrm{GeV} / \mathrm{c}$. It consists of a total of 2304 single wire drift cells grouped into 144 modules. Figure 3.17 (a) shows the position of the $\mathrm{CMU}$ detector in $\mathrm{r}-\phi$ and $\mathrm{r}-z$ projections with three modules matching a calorimeter tower of $15^{\circ}$. The instrumented region in a wedge spans $12.61^{\circ}$. A cross 


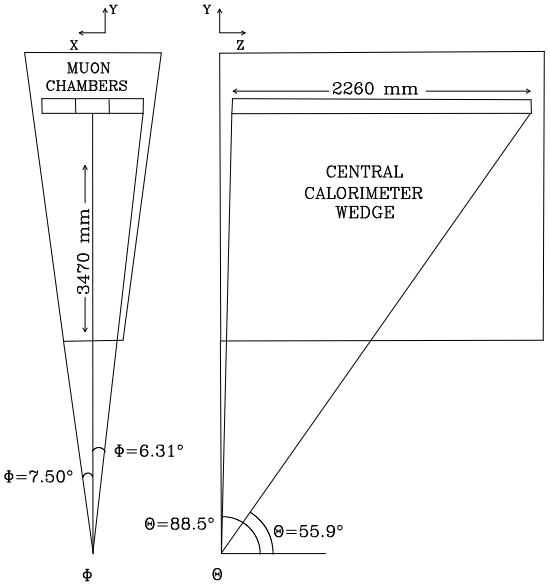

(a)

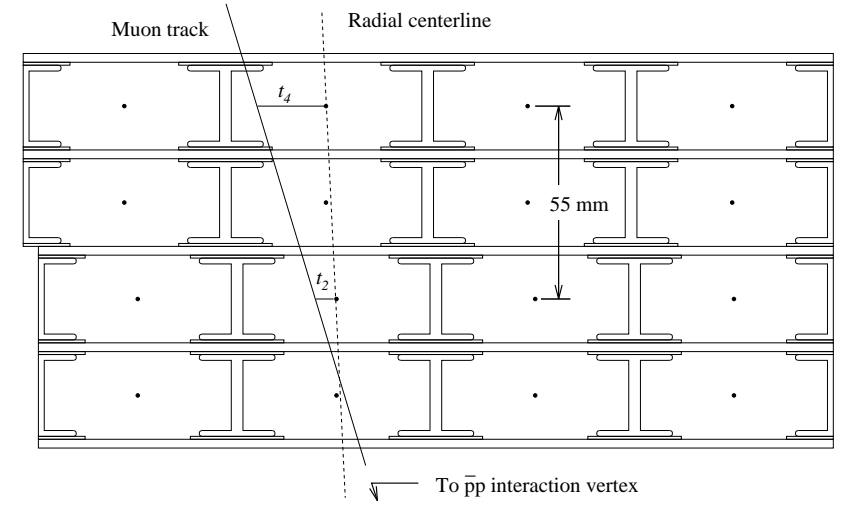

(b)

Figure 3.17: (a) Location of the central muon detector in $\mathrm{r}-\phi$ and $\mathrm{r}-z$. (b) Cross section of a CMU module showing its 12 drift tube cells. Four cells in $\mathrm{r}$ are called a muon tower.

section of one such module with its drift tube cells is shown in figure 3.17 (b). These cells are of dimension $2.68 \times 6.35 \times 226 \mathrm{~cm}$ with a $50 \mu \mathrm{m}$ stainless steel wire in the centre. The wire is held at $2.325 \mathrm{kV}$. Cathodes at the edge of each cell are held at $-2.500 \mathrm{kV}$.

As figure 3.17 (b) shows, alternating chambers are shifted slightly with respect to each other so that the combination of two alternating cells projects back to the interaction point. Comparing the drift time of two such cells allows a crude measurement of the track's momentum and can thus be used for triggering. Adjacent wires are grouped together for readout. To allow for the higher rates at Run II, the chambers are now operated in proportional mode instead of limited streamer mode resulting in a lower gain. To recover the gain loss new preamplifiers are installed. TDCs, which are pipelined due to the relatively long maximum drift time $\left(\mathrm{t}_{\max }=800 \mathrm{~ns}\right)$ which exceeds the bunch crossing time, provide a location in $\phi$. Analog to digital converters (ADCs) at each end of a cell provide a $z$ measurement using charge division.

\section{The Central Muon Upgrade}

The Central Muon uPgrade (CMP) was installed in 1992 mainly to enhance muon identification above hadronic background from punch through. The CMP is thus 


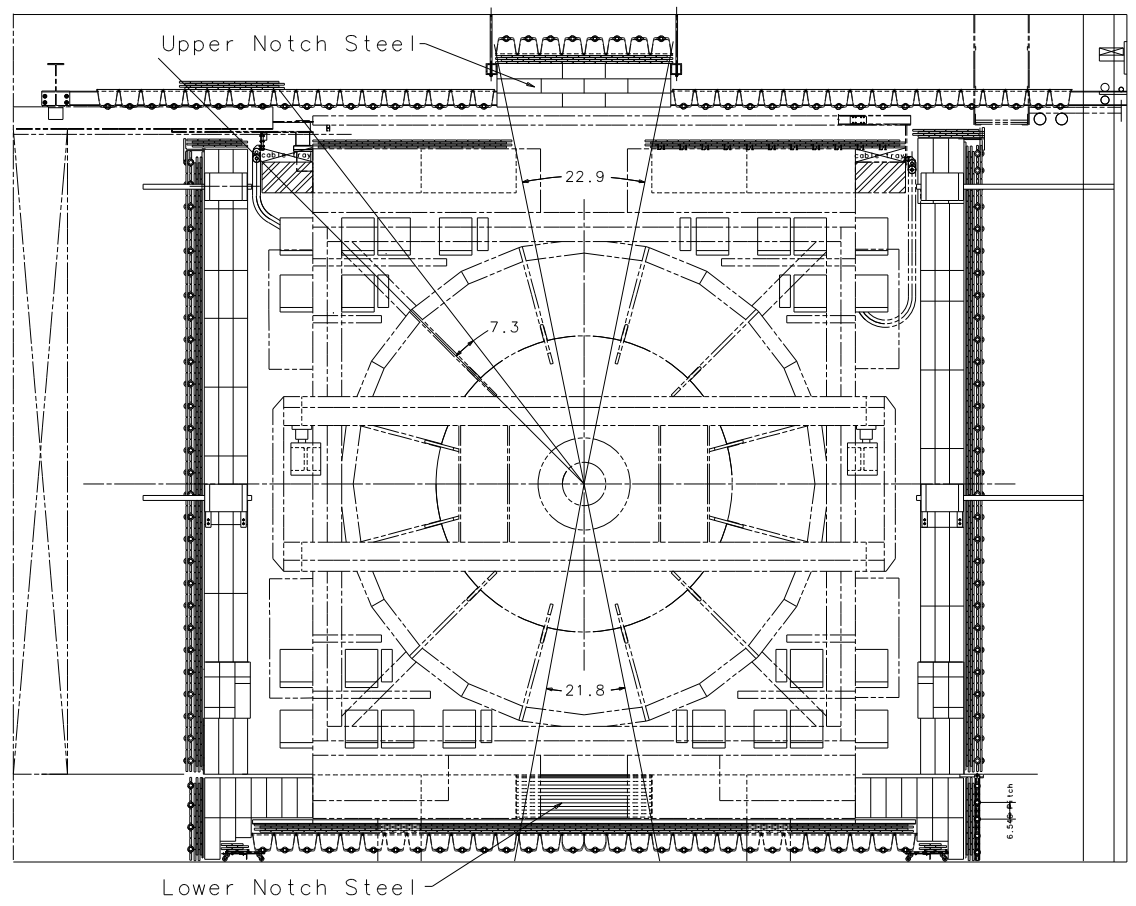

Figure 3.18: The dark grey square structure surrounding the central detector shows the configuration of the CMP and its scintillators. The structure of the steel absorber is also visible.

installed after an additional $60 \mathrm{~cm}$ of steel radially outwards from the CMU resulting, together with the calorimeter steel, in about 7.8 interaction length for pions. 1064 single wire drift cells are glued together to form 266 four layer stacks. The cells of adjacent layers are staggered with respect to each other with half cell spacing. Unlike most other detectors the CMP is not cylindrically shaped but forms a large rectangle around the $\mathrm{CMU}$ with a slightly complicated structure at top and bottom. This square structure is shown in figure 3.18.

This geometry, with a fixed length in $z$ results in $\phi$ dependent dips in the $\eta$ coverage which is visible in figure 3.16. The drift cells are $2.5 \times 15 \mathrm{~cm}$ and about $640 \mathrm{~cm}$ long (some are shorter due to space restrictions). Again they are single wire drift cells operated in proportional mode. The gold-plated tungsten wire is held at $+5.6 \mathrm{kV} .17$ cathode strips at the top and bottom of the cell shape the field of the (much wider than high) cells. The central strip is held at $3 \mathrm{kV}$, the voltage of the strips at either side is subsequently lowered by $375 \mathrm{~V}$ until they reach ground potential at the cell walls. Unlike the CMU, the CMP provides no $z$ information. The TDCs provide trigger information from coincidences of nearby 
wires in association with information from the CMU. A layer of 216 scintillation counters (CSP) surrounds the CMP to provide timing information. The counters measure $2.5 \times 15 \times 320 \mathrm{~cm}$ and are read out by single phototubes.

\section{The Central Muon Extension}

The Central Muon eXtension (CMX) was built for Run I and its $\phi$ coverage extended for Run II. It covers $0.6 \leq|\eta| \leq 1$. In Run I the top $30^{\circ}$ in $\phi$ of the west and east side were missing due to space restrictions from shielding for the main ring and the bottom $90^{\circ}$ were missing due to the floor of the collision hall. In Run II the shielding could be removed and the top $30^{\circ}$ at the west side are now instrumented. This part of the detector is called the key-stone. The east side contains cryogenic systems and remains uninstrumented as can be seen in figure 3.16. To extend the $\phi$ coverage the bottom $90^{\circ}$ were added for Run II with a slightly different geometry to the rest of the detector. This part is called the miniskirt, while the original components are called arches. The CMX arches lie on the surface of a virtual cone with opening angle of $41.4^{\circ}$ and have a vertex of about $10 \mathrm{~m}$ with respect to the interaction point. Figure 3.19 (a) indicates that design and shows the eight rows of chambers that extend outwards from the interaction point.

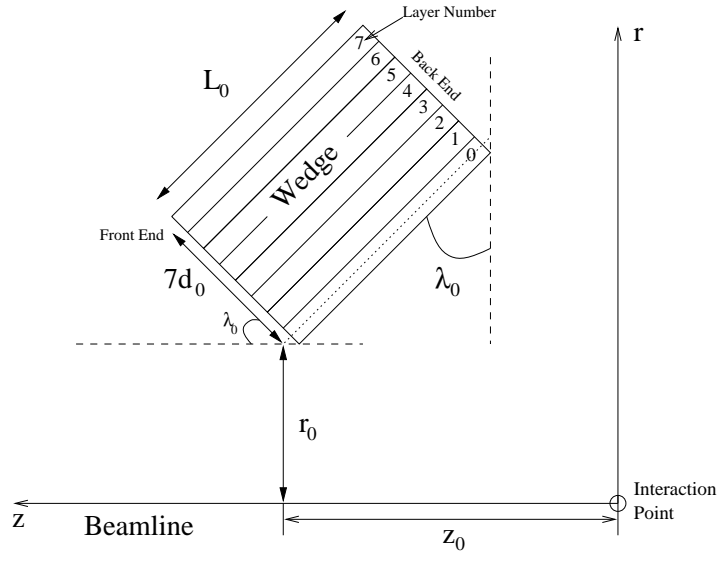

(a)

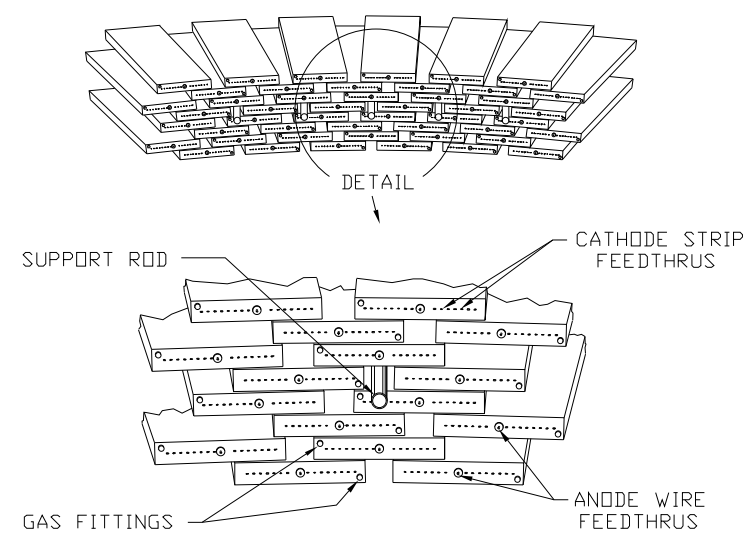

(b)

Figure 3.19: (a) Schematic $\mathrm{r}-z$ view of a CMX wedge. $\mathrm{L}_{0}=182.88 \mathrm{~cm}, \lambda_{0}=$ $48.57^{\circ}, \mathrm{d}_{0}=2.70 \mathrm{~cm}, \mathrm{z}_{0}=508.26 \mathrm{~cm}, \mathrm{r}_{0}=423.01 \mathrm{~cm}$. (b) Drift cell layout for the CMX. 
Each row contains six rectangular drift tubes per wedge, resulting in 48 chambers per $15^{\circ}$. The cells follow, apart from their length of $180 \mathrm{~cm}$, the same design as the CMP tubes. Figure 3.19 (b) shows the layout of the drift cells in one CMX module. The chambers of different layers overlap and the layout shown results in a $3.6 \mathrm{mrad}$ stereo angle between adjacent cells which can provide a polar angle measurement. The layers $n$ and $n+4$ are pointing back to the interaction point and differences in drift times on those pairs of wires are again used for triggering. Scintillation counters (CSX) are installed at the inner and outer surfaces of the cone to provide timing information. They are $2 \mathrm{~cm}$ thick and trapezoidal in shape with $27 \mathrm{~cm}$ width at the inner end and $45 \mathrm{~cm}$ width at the outer end. The counters are half staggered to improve the system's granularity. The miniskirts installed at the bottom $90^{\circ}$ are of similar design to the arches, but consist of a plane of chambers rather than a cone. They are equipped with one layer of scintillator (MSX) at the side closest to the interaction point. The key-stone design together with its scintillator mirrors that of the arches.

\section{Intermediate Muon Detector}

The new tracking system and the extended silicon system enable track reconstruction with $|\eta|>1$ and it is desirable to reconstruct and trigger on muons in the same region. The Run I muon system in the forward region has low granularity and would suffer from occupancy problems. It is thus replaced by a new detector for Run II, the Intermediate MUon detector (IMU). The IMU consists of CMP-like proportional drift tube arrays (BMU) and sets of scintillator (BSU and TSU) covering $1 \leq|\eta| \leq 2$. The barrel shaped BMU system is placed around large cylinders of steel absorber radially just inside the CMX. The absorber plates are based on the toroidal magnets of the forward muon spectrometer from Run I. Figure 3.20 shows the placement of the IMU system with respect to the CMX. 


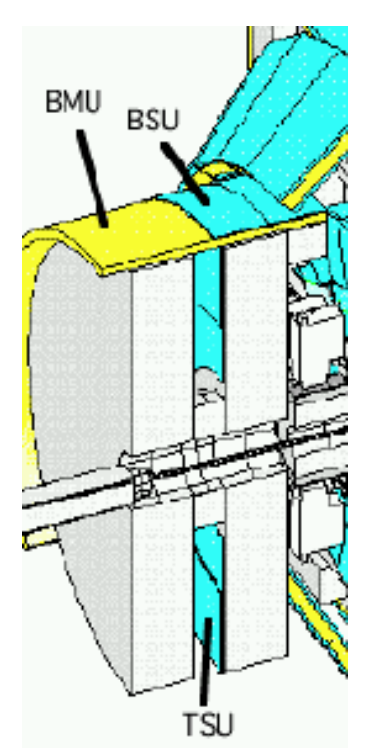

Figure 3.20: The intermediate muon system.

Due to the support structure for the absorber only the top $270^{\circ}$ are instrumented which can also be seen in figure 3.16. The $\mathrm{BMU}$ consists of four layers of drift chambers with dimensions $2.5 \times 8.4 \times 327 \mathrm{~cm}$, although some chambers are shorter due to space restrictions. Chambers two and four are staggered by half a cell with respect to chambers one and three respectively. Depending on the angle of incidence a particle will encounter 6.2 to $20 \lambda$ of material. The maximum drift time is $800 \mathrm{~ns}$. One layer of scintillator (BSU) surrounds the BMU tubes and a second system of scintillators (TSU) is embedded into the toroidal absorber. Both systems are indicated in figure 3.20. The scintillators of the latter system are trapezoidal in shape. The IMU can trigger on muons for $|\eta| \leq 1.5$ and identify offline muons for $|\eta| \leq 2$. 


\subsection{The Trigger}

A well designed trigger system is crucial for hadron collider experiments which have an event rate much higher than the rate with which data can be recorded to tape. At CDF II the event rate is effectively equal to the inverse of the bunch crossing time, resulting in 2.5 million events per second for the current bunch crossing time of $396 \mathrm{~ns}$. For the originally planned bunch crossing time of $132 \mathrm{~ns}$ this would increase to 7.6 million events per second. However at most 50 of those 2.5 million events can be written to tape. The purpose of the trigger is to select efficiently the most interesting events for further analysis whilst achieving the necessary reduction in the event rate. Here the definition of an interesting event depends on the specific physics goal, and multiple trigger paths are used to accommodate different needs.

The trigger system used in Run I was replaced for Run II to accommodate the higher luminosity and the reduction in bunch crossing time from $3.5 \mu \mathrm{s}$ in Run I to 132-396 ns. Figure 3.21 shows a diagram of the data flow through the three-level trigger system used in Run II. The first two levels comprise buffered and pipelined hardware triggers while the third level consists of an offline processing farm. Each level provides a sufficient rate reduction for processing in the next level with minimal deadtime.

Figure 3.22 shows in more detail which detector components and trigger subsystems contribute to the global trigger decisions at Level 1 (L1) and Level 2 (L2). Level 1 is a synchronous trigger, processing information from the calorimeters, the muon chambers and the COT simultaneously. It is fully pipelined with each detector element capable of buffering information from 42 events locally. With an estimated Level 1 decision time (latency) of $4000 \mathrm{~ns}$ and an additional safety margin of 1544 ns for unpredicted effects and transition time of trigger signals, a 42 event deep buffer is necessary to ensure that a new event can be read out every 132 ns. As shown in the block diagram of figure 3.22 the Level 1 trigger decision is based on a combination of information from the calorimeter, the central tracking chamber COT and the muon system. The most significant change compared to Run I is the availability of track finding information which was previously only available at Level 2. Based on hit information from the COT, tracks are reconstructed within $2.7 \mu \mathrm{s}$ 


\section{Dataflow of CDF "Deadtimeless" Trigger and DAQ}

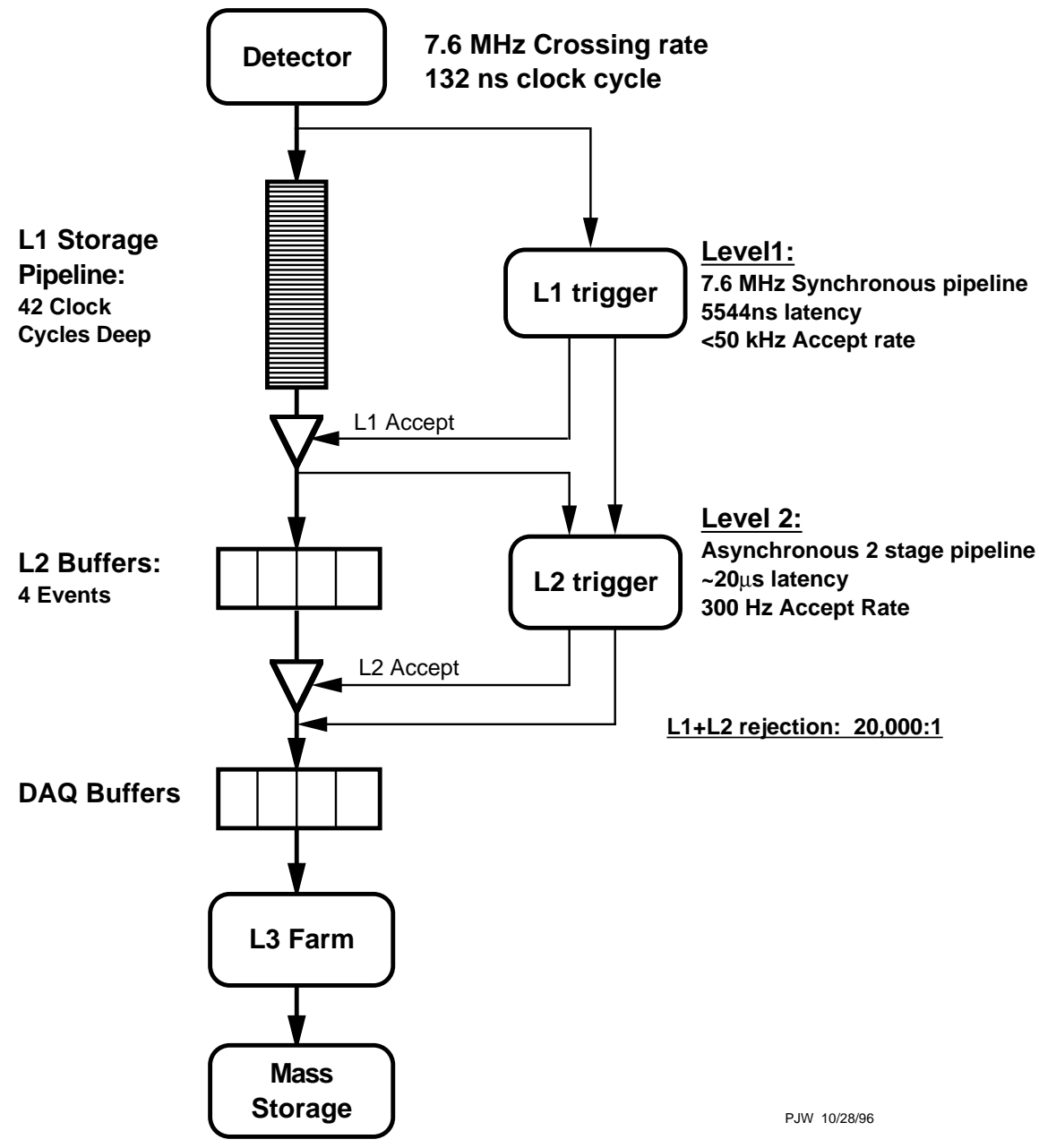

Figure 3.21: Architecture of the three-level pipelined and buffered trigger system for CDF II [38].

of a collision, which gave the XFT (eXtremely Fast Tracker) its name. The tracks are then passed to the XTRP (track eXTRaPolator), which selects tracks above $1.5 \mathrm{GeV} / \mathrm{c}$, extrapolates those tracks to the calorimeter and to the muon chambers and distributes this information or the tracks themselves to the L1 and L2 subsystems. The L1 calorimeter trigger (L1CAL) can then form calorimeter objects such as electrons, jets and photons, based on whether a calorimeter cluster has a matching track. The L1 muon trigger (L1MUON) similarly forms muon objects, while the L1 track trigger (L1TRACK) is based on information from the XFT (as the XTRP 


\section{RUN II TRIGGER SYSTEM}

Detector Elements

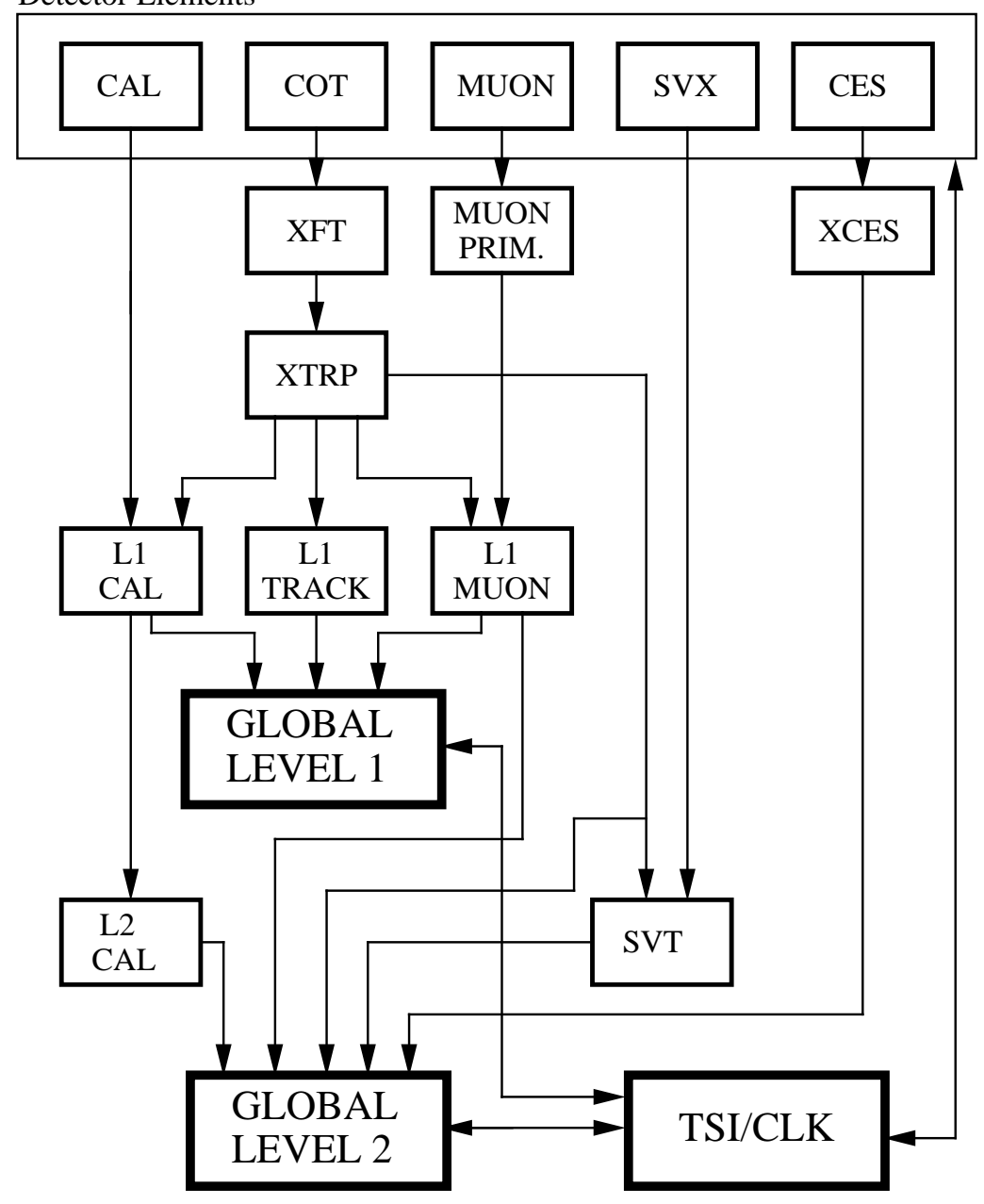

PJW 9/23/96

Figure 3.22: Block diagram of the trigger system for CDF II. The Trigger Supervisor Interface (TSI) distributes signals between the trigger and the data acquisition system such as the bunch crossing signal and Level 1 and Level 2 accept or reject signals.

here merely selects output tracks larger than $1.5 \mathrm{GeV} / \mathrm{c}$ ). An observation of more than four tracks generates an auto accept of the event; additionally the $\mathrm{p}_{\mathrm{T}}$ and $\phi$ information from tracks can be used for a trigger acceptance decision. The global Level 1 trigger then accepts or rejects and event based on the number and type of objects obtained from L1CAL, L1TRACK and L1MUON. 


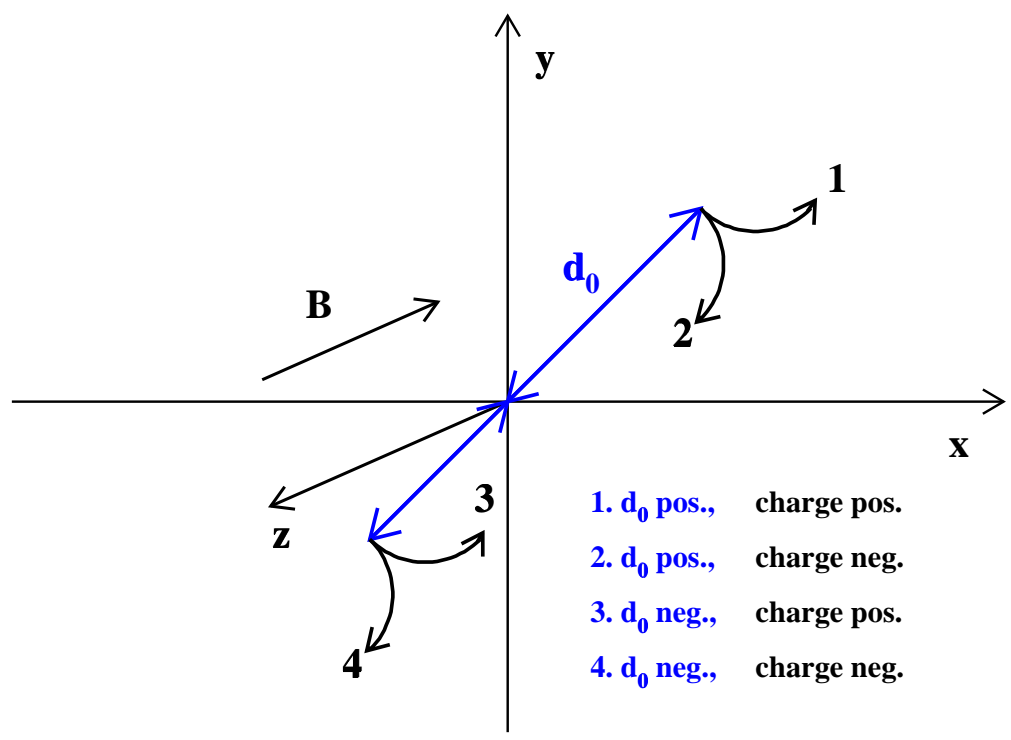

Figure 3.23: Definition of the impact parameter $d_{0}$ at CDF. The impact parameter is the signed distance between the track helix and the primary interaction vertex at the point of closest approach. The sign is the opposite sign to the angular momentum of the track. The magnetic field vector at CDF points in negative $z$ direction.

Upon a Level 1 accept all information is passed to the Level 2 subsystems. To allow an input rate of approximately $50 \mathrm{kHz}$ Level 2 has a buffer for four events and is pipelined, splitting the decision process into two separate stages, each of which takes about $10 \mu \mathrm{s}$. This yields a latency of $20 \mu \mathrm{s}$ but the effective time between two decisions is now $10 \mu$ s thus reducing the deadtime. In the first stage the event is built using information from the shower maximum detector (XCES) and the L1 triggers XFT and L1MUON. Simultaneously the second level calorimeter trigger L2CAL yields improved cluster finding and the SVT (Silicon Vertex Trigger) track processor finds tracks in the SVX II using hits from the $\mathrm{r}-\phi$ strips of the SVX II and tracks from the XFT. This collection of event data in the memory of the Level 2 processors takes about $10 \mu$ s after which the event is examined in stage two using Alpha processors based on the DEC Alpha chip to make a trigger decision. The event rate at Level 2 is reduced using the advanced jet cluster finding from L2CAL and the improved electron and photon identification achieved with the CES, which has better spatial resolution than the calorimeter itself. The most significant addition to 
Level 2 is however the SVT. In addition to an improved measurement of the $\phi$ and $\mathrm{p}_{\mathrm{T}}$ of a track it allows, for the first time in a hadron collider experiment, triggering on secondary vertices through the addition of a measurement of the track impact parameter $d_{0}$. The impact parameter of a track is defined as the signed distance between the track helix and the primary interaction vertex at the point of closest approach. The sign of the impact parameter is defined at CDF as being of opposite sign to the angular momentum $(\vec{r} \times \vec{p})$ of the track in question. The coordinate system with respect to which the position vector $\vec{r}$ is determined is chosen such that its origin coincides with the primary interaction vertex. Figure 3.23 shows four tracks with their signed impact parameters to demonstrate this definition. It can also be seen that the charge is irrelevant for the sign of the impact parameter.

This new trigger is of great importance to the B physics program at CDF as hadrons can travel a distance of several hundred microns before they decay and can thus be identified via impact parameters significantly different from zero. The two track trigger (TTT) for instance triggers on two displaced tracks and is used to obtain a sample of fully hadronic decays for measurements of branching ratios and mixing analyses. The trigger used in the analysis presented here, the lepton-SVT trigger, which selects events with one displaced track and a lepton is described in more detail in the following subsection.

Upon a Level 2 accept the full detector information collected from the front end electronics is read out by the Data AcQuisition system (DAQ) and sent to Level 3, a farm of offline processors. Here the entire event is built and sophisticated trigger decisions, based for instance on full 3D track reconstruction, are made. Upon a Level 3 accept the event is written to mass storage.

\section{The Lepton-SVT Trigger}

The trigger used for the selection of $\Lambda_{b}$ candidates in this analysis is the lepton-SVT trigger (lsvt) which itself is divided into two streams depending on whether the lepton is an electron (esvt) or a muon (musvt). The main requirements for event selection at the various trigger levels are listed below. Whenever a condition is only required for the electron or muon case this is indicated in brackets. 
- Level 1: single $4 \mathrm{GeV}$ lepton

$-\geq 4 \mathrm{GeV} / \mathrm{c}$ transverse momentum as determined by the XFT

$-\geq 4 \mathrm{GeV}$ energy in the calorimeter (e)

- had/em $<0.125$ (ratio of the energy deposited in the hadronic calorimeter and the electromagnetic calorimeter)

- muon object with $\geq 3 \mathrm{GeV} / \mathrm{c}$ transverse momentum in the CMP $(\mu)$

- Level 2: $4 \mathrm{GeV}$ lepton $+2 \mathrm{GeV}$ SVT track

$-\mathrm{SVT} \mathrm{p}_{\mathrm{T}} \geq 2 \mathrm{GeV} / \mathrm{c}$

$-120 \mu \mathrm{m} \leq \mathrm{d}_{0} \leq 1 \mathrm{~mm}$

$-\geq 4 \mathrm{GeV} / \mathrm{c}$ XFT track

$-\mathrm{E}_{\mathrm{T}}(\mathrm{CES}) \geq 2 \mathrm{GeV}(\mathrm{e})$

$-\geq 4 \mathrm{GeV}$ energy in the calorimeter (e)

$-\mathrm{had} / \mathrm{em}<0.125$

$-2^{\circ}<\Delta \phi(\mathrm{e}-\mathrm{SVT})<90^{\circ}(\mathrm{e})$

- Level 3:

- more stringent cuts on the electron and muon identification, for instance position difference in $\mathrm{r}-\phi$ between a track extrapolated to the muon chambers and a muon hit $<15 \mathrm{~cm}$

- invariant mass of the lepton and SVT track $<5 \mathrm{GeV} / \mathrm{c}^{2}$

$-2^{\circ}<\Delta \phi(1-\mathrm{SVT})<90^{\circ}$ for electron and muon

The cut values for the trigger were motivated by a study [39] that uses MC simulation of the decays $B_{s} \rightarrow D_{s} l \nu$ and $\Lambda_{b} \rightarrow \Lambda_{c} l \nu$ as well as Run I test-run data to optimise the signal-to-background ratio while at the same time trying to keep the signal selection efficiency as high as possible. 


\section{Chapter 4}

\section{Event Reconstruction and Selection}

The aim of the analysis described in this thesis is to measure the lifetime of the $\Lambda_{b}$ baryon in its semileptonic channel $\Lambda_{b}^{0} \rightarrow \Lambda_{c}^{+} \mu^{-} \bar{\nu}_{\mu}$, where the $\Lambda_{c}^{+}$subsequently decays to $p K^{-} \pi^{+1}$. The Feynman diagrams for the two decays are shown in figure

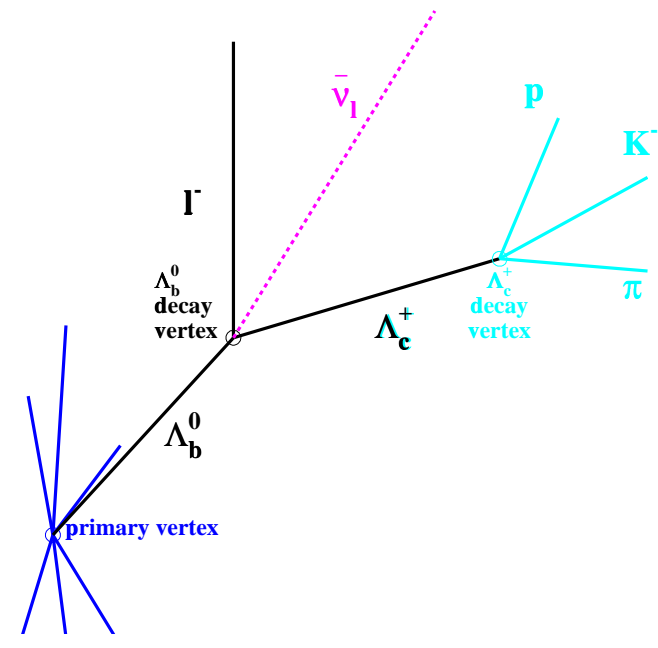

Figure 4.1: Event topology for the decay $\Lambda_{b}^{0} \rightarrow \Lambda_{c}^{+} l^{-} \bar{\nu}_{l}$ with the subsequent decay $\Lambda_{c}^{+} \rightarrow p K^{-} \pi^{+}$. The decay length of the $\Lambda_{c}$ is highly exaggerated.
2.15. The final state of this decay chain is characterised by the existence of a lepton from the $\Lambda_{b}$ decay and three tracks from the $\Lambda_{c}$ decay that form a displaced vertex in the vicinity of the lepton. This event topology is indicated in figure 4.1, with an exaggerated decay length for the $\Lambda_{c}$ baryon: the mean decay length of the $\Lambda_{c}$ is approximately one sixth of the mean decay length of the $\Lambda_{b}$. The neutrino is indicated by a dashed line as it does not leave a signal in the CDF detector.

As mentioned in the previous chapter, CDF II is equipped with a specialised trigger to select semileptonic events of this type. To recapitulate, the trigger requirements are a lepton object with a transverse momentum above $4 \mathrm{GeV} / \mathrm{c}$ and an additional track found by the SVT with $2^{\circ}<\Delta \phi(\mathrm{l}-\mathrm{SVT})<90^{\circ}, \mathrm{p}_{\mathrm{T}} \geq 2 \mathrm{GeV} / \mathrm{c}$, invariant mass $(1-\mathrm{SVT})<5 \mathrm{GeV} / \mathrm{c}^{2}$ and most importantly $120 \mu \mathrm{m} \leq \mathrm{d}_{0} \leq 1 \mathrm{~mm} ; \mathrm{d}_{0}$

\footnotetext{
${ }^{1}$ The measurement in this thesis treats the semileptonic decay to muons. However, for greater generality, the muons in diagrams and figures and related discussions are often replaced by leptons.
} 

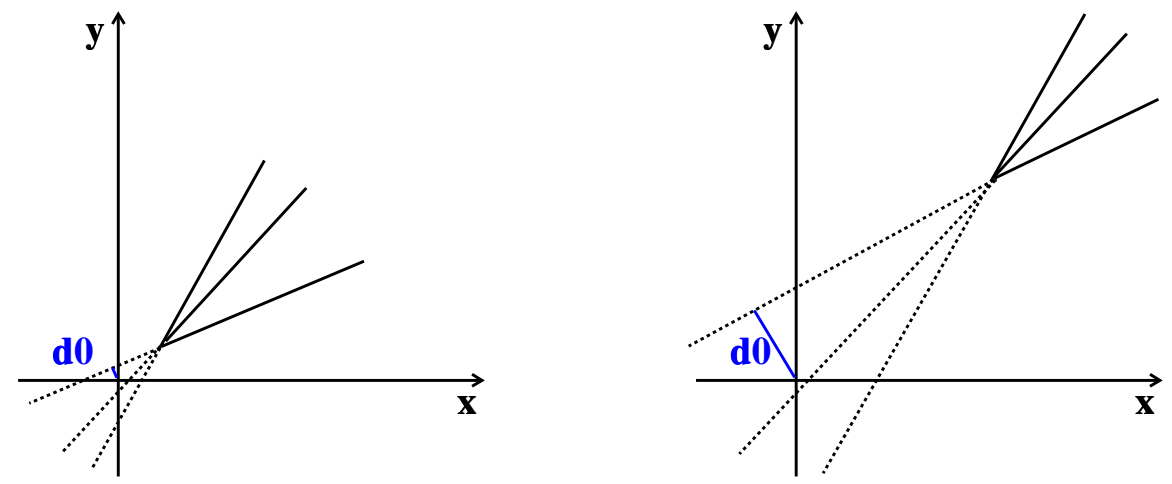

Figure 4.2: Impact parameter $d_{0}$ of a track shown for two kinematically equivalent decays with different lifetimes.

is the track impact parameter as introduced in figure 3.23. Figure 4.2 illustrates that the further out a given type of decay vertex lies, the larger is the impact parameter of the tracks originating at that vertex - with the exception of tracks that point back to the origin. Thus this trigger enhances events that contain particles that travel a few hundred microns before they decay, making it a very valuable trigger for B physics at hadron colliders.

The reconstruction of events from signals recorded in the various subdetectors comprises the reconstruction of tracks from hits, the reconstruction of decay vertices from tracks, the reconstruction of electromagnetic objects from entries in the calorimeters and muon objects from entries in the muon chambers and finally the combination of information from various subdetectors to identify particle species.

\subsection{Track and Vertex Reconstruction}

The track reconstruction starts, in most cases, with hits left by a particle in the central tracking chamber. The ionisation caused by charged particles passing through the COT volume is collected by its sense wires and the COT hardware ultimately provides a measurement of the drift time for a detected hit. The hit position is then calculated from the wire position, the drift time and the drift velocity. At this stage there is a \pm ambiguity as the drift time measurement of a single hit does not 


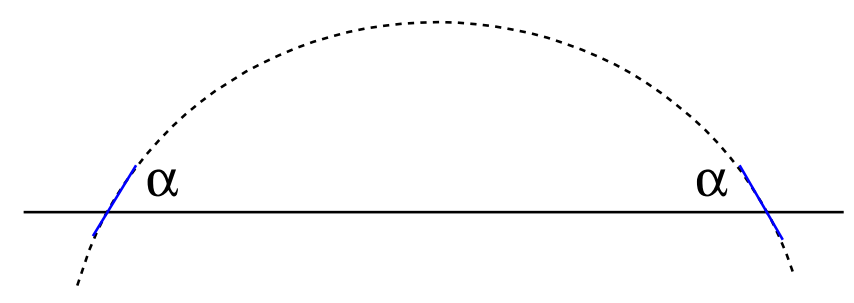

Figure 4.3: Two track segments (blue, short lines) and the angle $\alpha$ formed between the line connecting their centres and the circumference to which both segments are tangent.

determine which side of the sense wire the track passed. This is an issue resolved during track finding.

Once all COT hit candidates in the event are known, the eight superlayers are scanned for line segments, defined as triplets of unused aligned hits which belong to consecutive layers. A list of candidate segments is then formed and ordered by increasing slope of the segment with respect to the radial direction and the hit positions of a segment are fitted to a straight line. The fitted line segments are the starting point for two different track finding algorithms, segment linking SL [40], which was already used in Run I, and histogram linking HL, which was added for Run II to recover tracks that fail the SL algorithm. The idea behind the two methods is briefly described below. A more detailed description can be found elsewhere [41].

Segment linking is done in two steps, joining axial segments to 2D tracks and then adding stereo segments and hits to form 3D tracks if possible. For the axial SL the outermost axial segments are taken as seeds. Assuming the track impact parameter is zero, they are then extrapolated to the beam pipe. The intersection of this circle with the next superlayer serves as the centre of a search region, extending $1 \mathrm{~cm}$ to either side. All segments within this search region are then compared to the initial segment. Figure 4.3 shows two segments (blue lines) that belong to the same track and thus are tangent to the same circumference. The angle $\alpha$ indicated in the figure should be the same for both segments up to a finite resolution. If that is the case for two segments, they are linked and the linked segments are used as seeds now releasing the $\mathrm{d}_{0}=0$ constraint. Once all possible axial segments are linked the tracks are fitted to circles in the transverse plane and then linked to stereo segments to ultimately form 3D tracks. 
Histogram linking on the other hand is a reconstruction algorithm based on single hits and is applied in the axial track reconstruction. Instead of using segments of all superlayers only the outermost segments are used and serve as a "telescope", a pointer in the direction of the correct position and parameters of a track to which single hits, that do not necessarily belong to a complete segment, are assigned. In the algorithm the track parameters are then fixed apart from one. At CDF this free parameter is chosen to be the track's curvature. The curvature of the track is then varied within a specified search region. The likelihood for a hit to belong to a track with a certain curvature is then calculated and from the combined likelihood of all hits the best curvature and the hits belonging to the track with this curvature are found.

Analogously to finding hits in the COT through ionisation collected by the sense wires, tracking in the Silicon devices starts by forming hits from the charge deposited on the Silicon strips. Although it is possible at CDF to find tracks purely with the Silicon detectors (Silicon stand-alone tracking) most particles are reconstructed using COT tracks as seeds with the subsequent addition of Silicon information. More information on standalone tracking, as well as details of the COT seeded track reconstruction in the Silicon detectors, can be found elsewhere $[41,42]$.

Once tracks are reconstructed, the vertices at which tracks originate can be reconstructed. Apart from the primary event vertex there are two decay vertices which are important for the present analysis (see figure 4.1): the $\Lambda_{b}$ decay vertex at which the lepton, the neutrino and the $\Lambda_{c}$ baryon are created, and the $\Lambda_{c}$ decay vertex from which a proton, a kaon and a pion emerge. At the latter vertex all three particles are charged and thus detectable, at the former vertex the neutrino escapes detection while the $\Lambda_{c}$ decays too quickly for a track to be reconstructed. The procedure here is to find the $\Lambda_{c}$ vertex and to determine the resulting track parameters of the tracks originating at that vertex. Combining those tracks to a pseudo $\Lambda_{c}$ track, this track can be used together with the lepton track to obtain the $\Lambda_{b}$ vertex.

The vertex reconstruction at $\mathrm{CDF}$ is based on the module VertexFit which is a $\mathrm{C}++$ wrapper of the vertex finder CTVMFT [43] used in Run I. A three dimensional 
track can be parametrised by five parameters which are, in the case of CDF, chosen to be:

- $d_{0}$, the track's impact parameter

- $z_{0}$ and $\phi_{0}$, the $z$ and $\phi$ position of the track at the point of closest approach to the $z$-axis

- $\lambda=\cot \theta$, where $\theta$ is the polar angle of the track in radian

- $c=\frac{1}{2 r}$, the half-curvature of the track.

The length along the track, $s$, can be transformed into a $\phi$ value through

$$
\phi(s)=\frac{s}{r}+\phi_{0}=2 c s+\phi_{0}
$$

and the $x, y$, and $z$ coordinate of the track are obtained for any $s$ (or $\phi$ ) through the following equations:

$$
\begin{gathered}
x=r \sin \phi-\left(r+d_{0}\right) \sin \phi_{0} \\
y=-r \cos \phi+\left(r+d_{0}\right) \cos \phi_{0} \\
z=z_{0}+s \lambda .
\end{gathered}
$$

Equations 4.1-4.3 can be solved for $s, d_{0}$ and $z_{0}$ :

$$
\begin{gathered}
s=\frac{1}{2 c} \sin ^{-1}\left[2 c\left(x \cos \phi_{0}+y \sin \phi_{0}\right)\right] \\
d_{0}=y \cos \phi_{0}-x \sin \phi_{o}-\frac{1}{c} \sin ^{2} c s \\
z_{0}=z-\lambda s .
\end{gathered}
$$

The goal of the vertex fit is now to find the vertex point $\left(x_{s}, y_{s}, z_{s}\right)$ together with the fitted track parameters, so that the vertex lies on the respective tracks, i.e. that equations 4.4-4.6 hold for each track when inserting $x_{s}, y_{s}$ and $z_{s}$. The procedure applied in VertexFit to find such a vertex point is a minimisation of the following $\chi^{2}$ :

$$
\chi^{2}=\Sigma_{i=1}^{N} \xi_{i}^{T} G_{i}^{-1} \xi_{i}
$$


$\mathrm{G}$ is the error matrix of the measured parameters, and $\xi$ is a vector that contains the differences between the track parameters as constrained by equations 4.4-4.6 and the measured parameters: $\Delta c_{i}, \Delta \phi_{i}, \Delta \lambda_{i}, \Delta d_{i}$ and $\Delta z_{i} . N$ is the number of fitted tracks. While $\Delta d_{0}$ and $\Delta z_{0}$ are constrained according to equations 4.4-4.6, $\Delta c_{i}, \Delta \phi_{i}, \Delta \lambda_{i}$ as well as $x_{s}, y_{s}$ and $z_{s}$ are free parameters of the fit. More details about the fitting procedure as well as additional material on adding mass or pointing constraints to the fit can be found in the dedicated CDF note [43]. The vertex fit probability, calculated from the $\chi^{2}$ value of the fit, is required to be larger or equal to 0.001 in this analysis. Distributions for the fit probability for data and Monte Carlo events are shown in section 4.4 .

\subsection{Particle Identification}

Different types of particles leave different signatures in the various subsystems of the CDF detector, which can thus be used to discriminate amongst them. The calorimeters, together with the tracking system, provide information to distinguish between hadrons, electrons and photons. While electrons and hadrons yield a different shower shape in the calorimeter, photons are characterised by a calorimeter cluster without an associated track. Muons on the other hand are identified by an entry in the muon system that has a track associated with it. Furthermore the COT provides information on the specific energy loss, $\mathrm{dE} / \mathrm{dx}$, of a particle. This information is, like information from the TOF system, predominantly used to distinguish kaons, pions and protons from one another. The TOF system, described in section 3.2.2, is at present not used for this analysis. The various types of particle identification important for this thesis are described below.

\subsubsection{Muon Identification}

Muons are charged particles that leave a track in the tracking system as well as the muon system. They traverse the calorimeter as minimum ionising particles. The muon identification is thus based on matching a track reconstructed by the tracking system to hits in the muon chambers. The track is extrapolated to the respective 
muon system taking into consideration possible angular deviations caused by multiple scattering, and the difference $\Delta \mathrm{X}$ in $\mathrm{r}-\phi$ between this extrapolated position and the reconstructed muon object provides a variable to identify a muon. In this analysis only muons with $|\eta| \leq 0.6$, i.e. muons that pass through the central part of the muon system, the CMU and the CMP, are used and the criteria for identification as a muon are $\Delta \mathrm{X}_{\mathrm{CMU}} \leq 15 \mathrm{~cm}$ and $\Delta \mathrm{X}_{\mathrm{CMP}} \leq 20 \mathrm{~cm}$.

\subsubsection{Particle Identification via $\mathrm{dE} / \mathrm{dx}$}

Interactions with matter experienced by a charged particle include inelastic collisions with the atomic electrons, elastic collisions with their nuclei, Cerenkov radiation, nuclear reactions and bremsstrahlung. Heavy $\left(\mathrm{m} \gg \mathrm{m}_{\mathrm{e}}\right)$ charged particles experience mainly inelastic collisions which can be hard or soft, leading to ionisation or nuclear excitation respectively. The energy loss in such interactions is a statistical process; however, if the number of collisions is large, the average energy loss per unit path length $\mathrm{dE} / \mathrm{dx}$ is a useful quantity for particle identification.

A formula to calculate $\mathrm{dE} / \mathrm{dx}$ was first derived by Bohr from classical arguments and later refined by Bethe and Bloch using quantum mechanics. For heavy particles the mean rate of energy loss is given by the Bethe-Bloch formula [1] as:

$$
-\frac{d E}{d x}=4 \pi N_{A} r_{e}^{2} m_{e} c^{2} \frac{Z}{A} \frac{z^{2}}{\beta^{2}}\left[\frac{1}{2} \ln \frac{2 m_{e} c^{2} \beta^{2} \gamma^{2} T_{\max }}{I^{2}}-\beta^{2}-\frac{\delta}{2}\right] .
$$

$Z$ and $A$ are the atomic number and atomic mass of the absorber, $z$ is the charge of the incident particle in units of the electron charge, $r_{e}$ is the classical electron radius and $N_{A}, m_{e}, \beta, \gamma$ and $c$ have their usual meaning. $I$ is the mean excitation energy of the material and $T_{\max }$ is the maximum kinetic energy that can be transferred to the electron in a single interaction:

$$
T_{\max }=\frac{2 m_{e} c^{2} \beta^{2} \gamma^{2}}{1+2 \gamma m_{e} / M+\left(m_{e} / M\right)^{2}} .
$$

The small correction factor $\delta$ accounts for density effects: the electric field of the incident particle polarises atoms transverse to its path so that distant electrons are shielded from its full electric field. The density effect becomes more important at higher velocities of the incident particle, with more distant collisions adding to the 


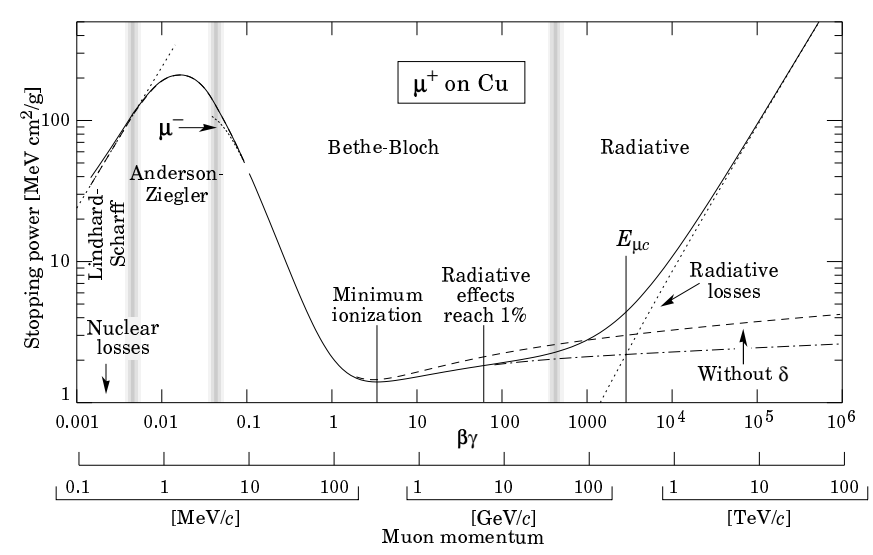

(a)

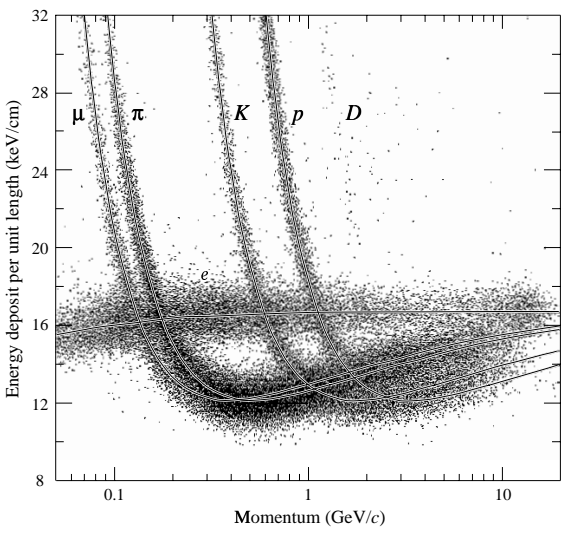

(b)

Figure 4.4: Figure (a) shows the stopping power for positive muons in copper as a function of $\beta \gamma$ [1] and figure (b) shows the energy deposited per unit length as a function of momentum for particles traversing the time projection chamber at the PEP accelerator [1].

total energy loss, and in dense materials such as lead and iron. Sometimes the Bethe-Bloch formula is quoted with the additional correction term $-2 \frac{C}{Z}[44]$ added to the term in brackets in equation 4.7. This term accounts for effects that arise when the velocity of the incident particle is comparable or lower than the orbital velocity of the bound electrons of the absorber and is referred to as shell correction.

Equation 4.7 shows that the mean energy loss is, apart from a slight dependency on the incident particle's mass introduced via $T_{m a x}$, only a function of $\beta \gamma$ and as such independent of the particle type. The $\mathrm{dE} / \mathrm{dx}$ versus $\beta \gamma$ curve is thus referred to as a universal curve and is shown for muons in copper in figure 4.4 (a) [1]. If $\mathrm{dE} / \mathrm{dx}$ is plotted against the particle's momentum, distinct bands can be recognised that show the separation power $[45,46]$ between different particle species depending on their momenta. Such a $\mathrm{dE} / \mathrm{dx}$ versus momentum measurement, typical of particles traversing gas-filled detectors, is shown in figure 4.4 (b) [1].

With its large number of sense wires, the COT provides the opportunity to measure the specific energy loss of traversing particles. When a charged particle traverses the drift cells of the central tracking chamber it ionises the gas along its path. The amount of ionisation produced in each cell is then measured via the charge collected with the sense wires of the COT. This yields an analog signal which is then transformed into a digital pulse so that the amount of charge is ultimately 
encoded in the width of the digital pulse and measured in ns. The distribution of the ionisation electron energy has a long tail. This results in big fluctuations of the $\mathrm{dE} / \mathrm{dx}$ measurement for different tracks if all hits are considered for the averaging. CDF employs the standard technique of using only the lowest $80 \%$ of the hits for the $\mathrm{dE} / \mathrm{dx}$ measurement, which is then referred to as the truncated mean. Several corrections, for instance concerning non-uniformities in the drift field, are applied $[45,46]$. In this analysis loose cuts on $\mathrm{dE} / \mathrm{dx}$ are applied to remove part of the background from wrongly identified proton candidates.

\subsection{Offline Confirmation of the Trigger Cuts}

The events that pass all levels of the trigger are reconstructed offline from information from all subdetectors and are stored with reconstructed tracks and lepton objects available for user analysis. The first step of the analysis is then to confirm offline all the trigger cuts that were applied online.

The offline confirmation is done with the validated module LeptonSvtSel [47]. In addition to confirming the trigger cuts it creates a collection of leptons and a collection of SVT tracks that fulfil the trigger requirements. These collections are then available for subsequent analysis. The trigger requirements that are repeated by default for muons are:

- $\mathrm{p}_{\mathrm{T}} \geq 4 \mathrm{GeV} / \mathrm{c}$

- $\Delta \mathrm{X}_{\mathrm{CMU}} \leq 15 \mathrm{~cm}$

- $\Delta \mathrm{X}_{\mathrm{CMP}} \leq 20 \mathrm{~cm}$

After the lepton (here muon) confirmation the SVT trigger tracks have to be matched to the offline reconstructed tracks. The quantity used to determine whether the tracks match is the $\chi^{2}$-like quantity [47]

$$
\chi_{\text {matching }}^{2}=\left(\frac{c^{\text {offline }}-c^{S V T}}{\sigma_{c}}\right)^{2}+\left(\frac{\Phi_{0}^{\text {offline }}-\Phi_{0}^{S V T}}{\sigma_{\Phi}}\right)^{2},
$$

where $c$ is the curvature of the track. For an offline track to be matched to an SVT track a $\chi^{2}$ smaller than 25 is required. All tracks that have a matching SVT track 
are stored at this point in a special track collection. Once a track is matched to an SVT track the standard track pair cuts concerning the angle $\Delta \phi$ between the lepton track and the SVT track and the invariant mass of this track pair combination, $2^{\circ}<$ $\Delta \phi(1-\mathrm{SVT})<90^{\circ}$ and $\mathrm{m}(\mathrm{l}-\mathrm{SVT})<5 \mathrm{GeV} / \mathrm{c}^{2}$, are applied. If one of the matched SVT tracks passes these cuts the event is kept for downstream analysis. Note that for the event to be accepted only one of the tracks in the collection of matched SVT tracks needs to pass the trackpair cuts and there is no information stored for subsequent analysis as to which tracks passed the cuts. As such the trackpair cuts are repeated in the user module. The efficiencies for the offline confirmation of muons, of the SVT-SVX matching and of the track pair cuts are approximately $90 \%, 43 \%$ and $35 \%$ respectively.

\subsection{Candidate Selection}

The studies for the candidate selection use $186 \mathrm{pb}^{-1}$ of data recorded between February 2002 and September 2003 with the mu+SVT trigger. This corresponds to a range of good runs from run number 138819 to run number 168889. A run is hereby classed as good, if the detector and online system components relevant for a specific trigger path were fully operational at the time the data was recorded. In the case of the mu+SVT trigger that includes, apart from the trigger systems such as the SVT trigger itself, mainly the tracking chambers and the central muon chambers.

\subsubsection{Preselection}

The events that pass the trigger have a multitude of origins. They include not just events from B-decays, and among the events from B-decays certainly not all comprise $\Lambda_{b}$ decays. The following applies to all $\Lambda_{b}$ signal events:

- $\mathrm{Q}(\mathrm{l}) \cdot \mathrm{Q}\left(\Lambda_{c}\right)=-1$, i.e. the lepton is of opposite charge to the $\Lambda_{c}$. The charge of the $\Lambda_{c}$ is obtained as the sum of the charges of the proton, the kaon and the pion track.

- $\mathrm{m}\left(\Lambda_{\mathrm{c}}\right) \leq \mathrm{m}\left(1 \Lambda_{\mathrm{c}}\right) \leq \mathrm{m}\left(\Lambda_{\mathrm{b}}\right)$, i.e. the invariant mass of the lepton- $\Lambda_{c}$ combination is at least the size of the invariant mass of the $\Lambda_{c}$ baryon and at most the size 
of the $\Lambda_{b}$ baryon. The actual value of $\mathrm{m}\left(1 \Lambda_{\mathrm{c}}\right)$ depends on the amount of momentum carried by the neutrino and the resolution.

The events that pass the trigger are first analysed with LeptonSvtSel, and $\Lambda_{b}$ candidates are obtained as follows. For every trigger lepton all tracks in its $\Delta \mathrm{R}$ vicinity $\left(\Delta \mathrm{R}=\sqrt{(\Delta \eta)^{2}+(\Delta \phi)^{2}} \leq 1.5\right)$ are analysed. The tracks are required to pass the following fiducial and quality requirements:

- $\mathrm{p}_{\mathrm{T}} \geq 0.4 \mathrm{GeV} / \mathrm{c},|\eta| \leq 1.1$

- minimum number of silicon $\mathrm{r}-\phi$ hits $=3$

- minimum number of hits in the axial layers of the COT $=20$

- minimum number of hits in the stereo layers of the COT $=20$

- minimum number of good stereo (axial) COT layers $=2$, where good means that there are at least 4 hits on the layer.

The tracks are then refitted to account for energy loss and multiple scattering which depends on the particle species; this is necessary as the standard tracks are all fitted using a pion hypothesis. During the refit the hits in the 90 degree and the small angle stereo layers of the silicon tracker are dropped. The hits in the innermost silicon layer, L00, and in the outermost silicon system, ISL, are not used in this analysis. At the time this analysis was performed the information from both detector systems was not approved for physics analysis since the available alignment was only preliminary and the L00 pedestals were not well understood.

Every possible three track combination with $\Sigma_{\mathrm{i}=1}^{3}\left(\mathrm{Q}_{\mathrm{i}}\right)= \pm 1$ is then investigated as a possible $\Lambda_{c}$ candidate and combined with a lepton. This lepton- $\Lambda_{c}$ combination is stored if $\mathrm{m}\left(\Lambda_{\mathrm{c}}\right) \leq \mathrm{m}\left(1 \Lambda_{\mathrm{c}}\right) \leq \mathrm{m}\left(\Lambda_{\mathrm{b}}\right)$ and if the maximal $z_{0}$ between the lepton and the three tracks is smaller than $2.5 \mathrm{~cm}$. To calculate the invariant mass of the $\Lambda_{c}$ candidate the tracks must be assigned a mass. From the charge assignments $\left(\Lambda_{c}^{+} \rightarrow p K^{-} \pi^{+}\right)$it follows that the track with opposite charge must be the kaon track and the like-sign tracks must be the proton and the pion. The assignment of the proton and pion mass, however, is ambiguous and both combinations can enter 


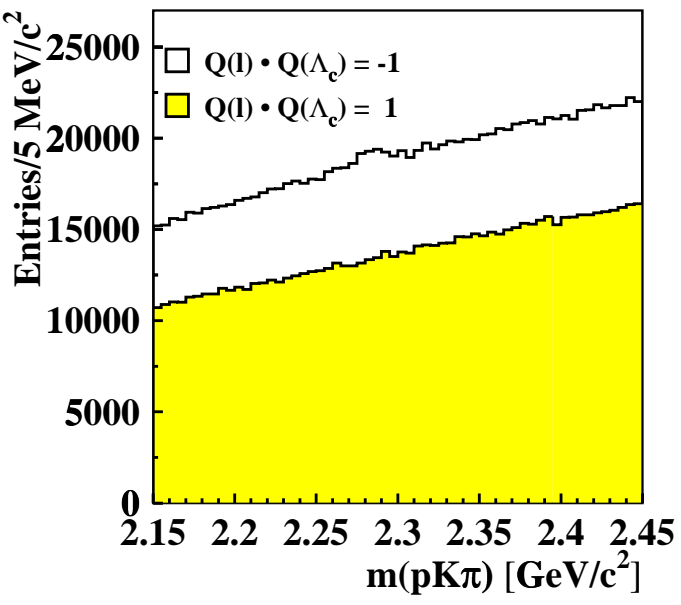

(a)

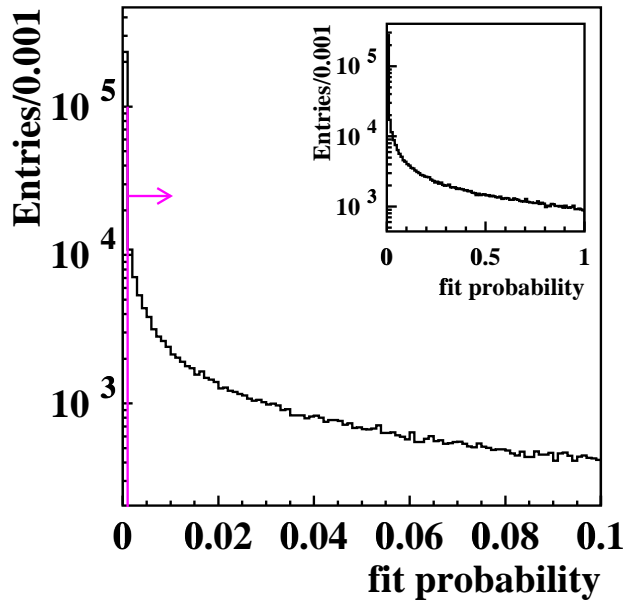

(b)

Figure 4.5: Figure (a) shows the $p K \pi$ invariant mass distribution for all three track combinations in the candidate collection after application of a cut on the fit probability at 0.001 . Figure (b) shows the distribution of the vertex fit probability for events with $2.26 \mathrm{GeV} / \mathrm{c}^{2} \leq \mathrm{m}(\mathrm{pK} \pi) \leq 2.32 \mathrm{GeV} / \mathrm{c}^{2}$. The cut value is indicated by the vertical line with an arrow pointing towards the accepted region.

the candidate collection. Both the right sign $\mathrm{Q}(1) \cdot \mathrm{Q}\left(\Lambda_{c}\right)=-1$ and the wrong sign $\mathrm{Q}(\mathrm{l}) \cdot \mathrm{Q}\left(\Lambda_{c}\right)=1$ combination are stored, which allows for monitoring of background and of any bias caused by further selection cuts.

Figure 4.5 (a) shows the invariant mass of all three track combinations in the candidate collection after application of a cut on the vertex fit probability at 0.001 . The white histogram shows the right-sign combination and the yellow/shaded histogram shows the wrong sign combination. Merely a hint of a $\Lambda_{c}$ signal is visible in the right-sign combination. The distribution of the vertex fit probability is shown in figure 4.5 (b) for events with $2.26 \mathrm{GeV} / \mathrm{c}^{2} \leq \mathrm{m}(\mathrm{pK} \pi) \leq 2.32 \mathrm{GeV} / \mathrm{c}^{2}$. The small plot in figure 4.5 (b) shows the whole range of probability values, and the enlarged histogram shows only a section to point out the location of the cut. The small size of the visible $\Lambda_{c}$ signal is largely due to the fact that the combinatorial background from forming three track combinations from all tracks with the correct charge assignment in a lepton's vicinity is very large.

There are various variables that discriminate the signal from background events. To optimise a set of selection cuts, distributions for cut variables are needed for 

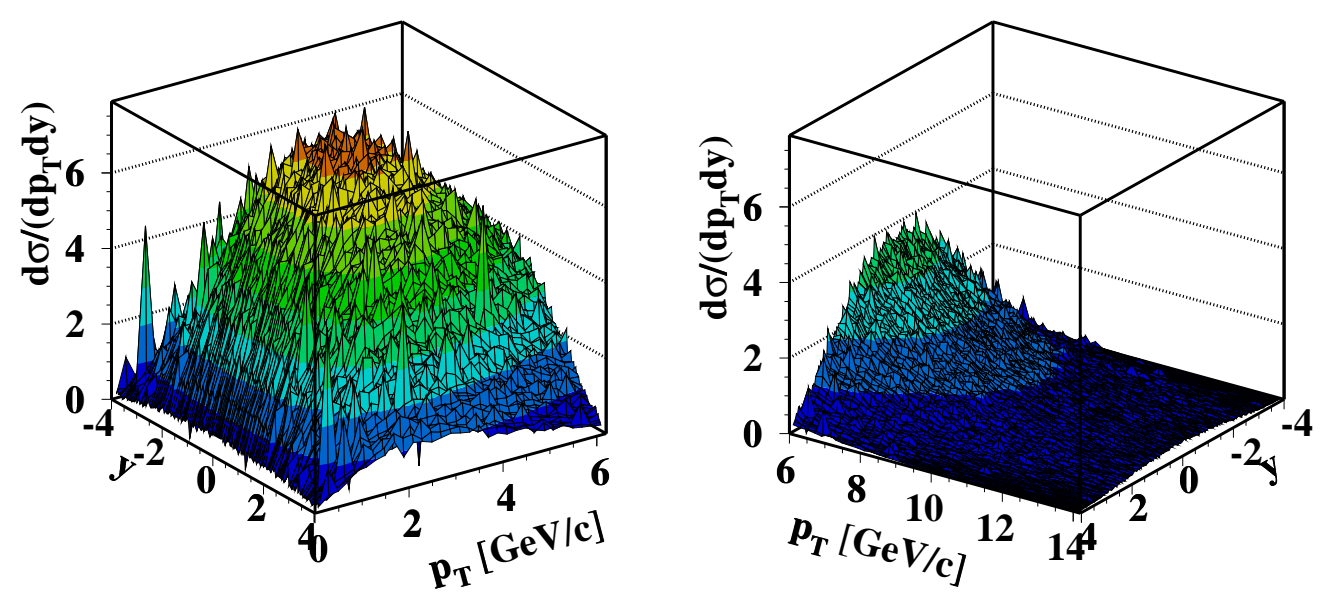
Figure 4.6: Input b quark $\frac{\mathrm{d} \sigma}{\mathrm{dp}_{\mathrm{T}} \mathrm{dy}}$ spectrum for the production of $\mathrm{B}$ hadrons
with Bgenerator.

the signal and the background. The background distributions are obtained using the sidebands of a prospective $\Lambda_{c}$ peak in the $p K \pi$ invariant mass plot. The signal distributions are predominantly obtained from simulated events to avoid biasing the invariant mass distribution through the selection cuts towards a fake $\Lambda_{c}$ signal. The cut selection will be described following a section on the Monte Carlo (MC) samples used in this analysis.

\subsubsection{Monte Carlo Simulation}

For the analysis two sets of MC are used, (1) a parametric simulation and (2) a realistic simulation. The parametric simulation, described below, is used to create input distributions for the lifetime fit; the realistic simulation is used to test the fit and optimise the selection cuts.

The generator level information for both types of MC is based on the Bgenerator [48] package for the production of B hadrons and on the CLEO decay package QQ [49] for the decay of the $\Lambda_{b}$ baryons and subsequent short lived particles. Bgenerator uses as input a b quark $\frac{\mathrm{d} \sigma}{\mathrm{dp}_{\mathrm{T}} \mathrm{dy}}$ spectrum which is shown in figure 4.6 for two momentum ranges. The range $0 \mathrm{GeV} / \mathrm{c} \leq \mathrm{p}_{\mathrm{T}} \leq 6 \mathrm{GeV} / \mathrm{c}$ is shown for completeness while only the range $\mathrm{p}_{\mathrm{T}} \geq 6 \mathrm{GeV} / \mathrm{c}$ is relevant for this analysis, due to the cuts on the transverse momenta of the lepton at $4 \mathrm{GeV} / \mathrm{c}$ and the SVT track at $2 \mathrm{GeV} / \mathrm{c}$. 


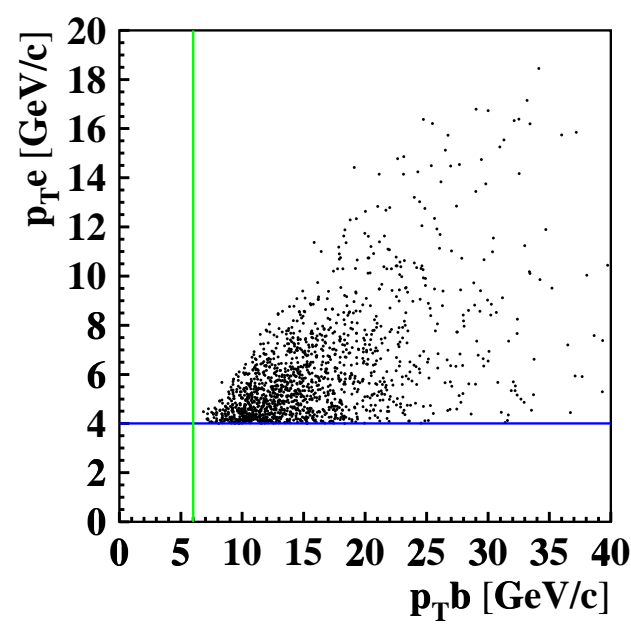

(a)

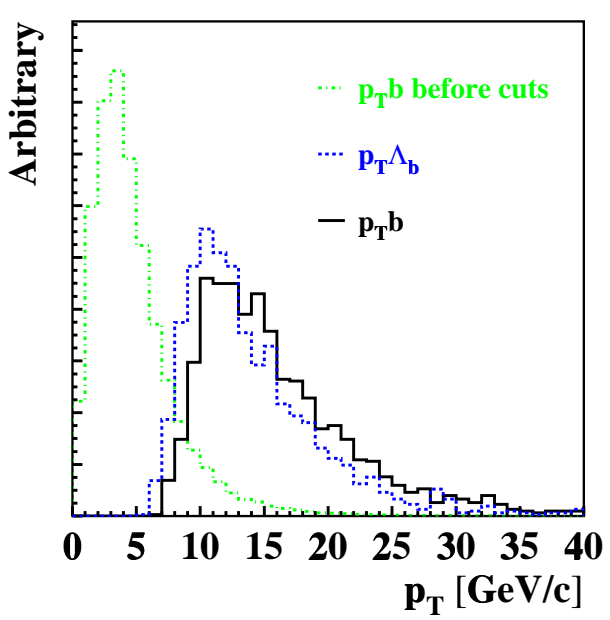

(b)

Figure 4.7: Figure (a) shows $\mathrm{p}_{\mathrm{T}} \mathrm{e}$ vs $\mathrm{p}_{\mathrm{T}} \mathrm{b}$ for the decay $\Lambda_{b} \rightarrow \Lambda_{c} e \nu, \Lambda_{c} \rightarrow p K \pi$ after a cut on the transverse momentum of the electron at $4 \mathrm{GeV} / \mathrm{c}$ and a cut on transverse momentum of one of $\mathrm{p}, \mathrm{K}$ or $\pi$ at $2 \mathrm{GeV} / \mathrm{c}$. Figure (b) shows the transverse momentum distributions for the $\mathrm{b}$ quark before cuts and for the $\mathrm{b}$ quark and the generated $\Lambda_{b}$ baryon after the two transverse momentum cuts.

Therefore, only b quarks with a transverse momentum above or equal to $6 \mathrm{GeV} / \mathrm{c}$ are generated. Figure 4.7 (a) shows the $\mathrm{p}_{\mathrm{T}}(\mathrm{l})$ versus $\mathrm{p}_{\mathrm{T}}(\mathrm{b})$ distribution for the decay $\Lambda_{b} \rightarrow \Lambda_{c} e \nu, \Lambda_{c} \rightarrow p K \pi$ after application of the $\mathrm{p}_{\mathrm{T}}$ cut for both the electron and one of the $\Lambda_{c}$ daughter tracks; no events are seen below a $\mathrm{p}_{\mathrm{T}}(\mathrm{b})$ of $6 \mathrm{GeV} / \mathrm{c}$. The $\mathrm{b}$ quark mass used for generation is $4.75 \mathrm{GeV} / \mathrm{c}^{2}$. Bgenerator uses the Peterson fragmentation described in 2.4.3. The fragmentation parameter $\epsilon$ is set to 0.006 (see figure 2.9). Figure 4.7 (b) shows the resulting distribution of the $\mathrm{p}_{\mathrm{T}}$ of the $\mathrm{b}$ quark before the transverse momentum cuts are applied, and the distributions of the $\mathrm{p}_{\mathrm{T}}$ of the $\mathrm{b}$ quark and the resulting $\mathrm{p}_{\mathrm{T}}$ of the $\Lambda_{b}$ baryon after application of the cuts.

The parametric simulation is based on the generator level momentum and vertex information of the tracks (which is stored in HEPG format), and quantities such as the decay length are calculated directly from this information. Certain detector effects, such as the tracking efficiency of the secondary vertex trigger are however accounted for. The details concerning the corrections applied to the parametric simulation can be found in chapter 5 . The realistic MC includes the simulation of the detector geometry with cdfSim [50] as well as a simulation of the trigger so that 


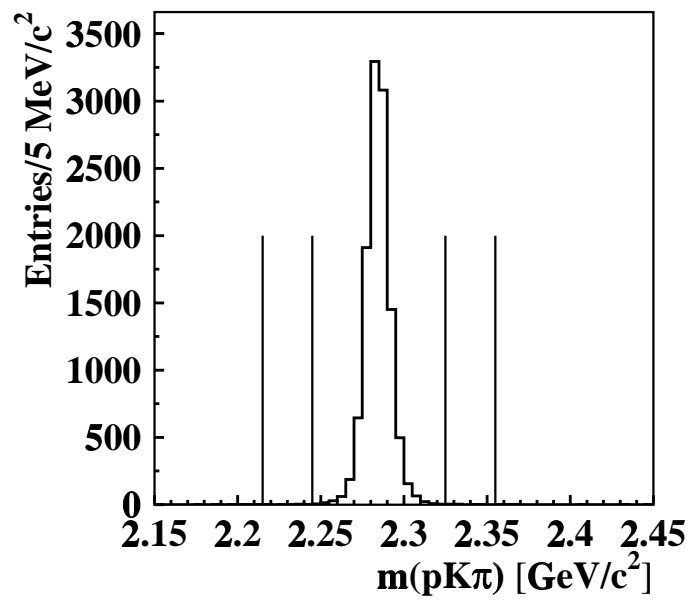

(a)

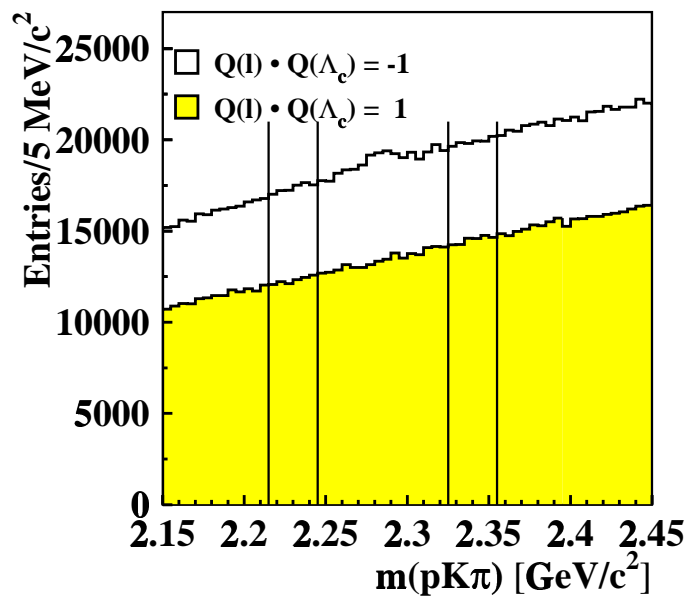

(b)

Figure 4.8: The $p K \pi$ invariant mass distribution for events from the realistic simulation (a) and from data after requiring the vertex fit probability to exceed 0.001 (b). The vertical lines indicate $3 \sigma$ wide sidebands located at $\pm(4-7) \sigma$ from a $\Lambda_{c}$ signal peak.

events from the realistic simulation can be analysed by the same algorithms that are used to analyse the data.

\subsubsection{Optimisation of Selection Cuts}

The optimisation of the selection cuts is done in several steps. Firstly, variables are identified that have some discriminating power between signal and background. The signal distributions are obtained from the realistic MC; the background distributions are obtained from data using events whose value of the $p K \pi$ invariant mass falls into $3 \sigma$-wide sidebands at either side of the prospective $\Lambda_{c}$ peak. These sidebands are shown in figure 4.8 (a) and (b). Figure (a) shows the $\Lambda_{c}$ peak obtained from the realistic simulation and figure (b) shows the $p K \pi$ invariant mass peak from figure 4.5 (b). The sidebands are separated from the $6 \sigma$-wide peak region at either side by $1 \sigma$ and thus are located at $\pm(4-7) \sigma$ from the centre of the signal peak.

The following variables are considered as cut variables for the signal selection:

1) variables concerning the difference in the $(\eta, \phi)$ separation $\Delta \mathrm{R}=\sqrt{(\Delta \eta)^{2}+(\Delta \phi)^{2}}$ of various track pairs:

- $\Delta \mathrm{R}_{\max }(\mathrm{pK} \pi)$, the maximum $\Delta \mathrm{R}$ of $\Delta \mathrm{R}(\mathrm{pK}), \Delta \mathrm{R}(\mathrm{p} \pi), \Delta \mathrm{R}(\mathrm{K} \pi)$ 
- $\Delta \mathrm{R}_{\max }(\mathrm{l}-\mathrm{pK} \pi)$, the maximum $\Delta \mathrm{R}$ of $\Delta \mathrm{R}(\mathrm{lp}), \Delta \mathrm{R}(\mathrm{lK}), \Delta \mathrm{R}(\mathrm{l} \pi)$

- $\Delta \mathrm{R}_{\min }(\mathrm{pK} \pi)$ and $\Delta \mathrm{R}_{\min }(\mathrm{l}-\mathrm{pK} \pi)$

- $\Delta \mathrm{R}\left(\mathrm{l}-\Lambda_{\mathrm{c}}\right)$, the difference in $\mathrm{R}$ between the lepton and the $\Lambda_{c}$ candidate

2) various kinematic variables:

- the transverse momenta of the proton, the kaon, the pion and the resulting $\Lambda_{c}$ baryon

- the transverse momentum of the lepton- $\Lambda_{c}$ combination

- the ratio of the transverse momenta of the proton and the pion, $\mathrm{p}_{\mathrm{T}} \mathrm{p} / \mathrm{p}_{\mathrm{T}} \pi$

- the invariant mass of the lepton- $\Lambda_{c}$ combination

3) variables concerning the lifetime or decay length measurement:

- $c \tau\left(\Lambda_{c}\right)$, calculated from the decay length of the $\Lambda_{c}$ baryon in the transverse plane, its mass and its transverse momentum; the decay length itself is returned from the vertex fit as the distance between the $\Lambda_{b}$ and the $\Lambda_{c}$ decay vertex

- $\mathrm{L}_{\mathrm{xy}}$ (p.v. $-\Lambda_{\mathrm{c}}$ ), the decay length in the transverse plane, returned by the fit as the distance between the primary vertex and the $\Lambda_{c}$ decay vertex

- $\mathrm{dL}_{\mathrm{xy}}\left(\Lambda_{\mathrm{b}}\right), \mathrm{dL}_{\mathrm{xy}}\left(\Lambda_{\mathrm{c}}\right)$, the error on the respective value of the decay length as returned by the vertex fit.

The distributions of these variables are shown in figures 4.9-4.11 after the application of the cut on the fit probability at 0.001 . The yellow/shaded histograms show the signal distributions which are obtained from the realistic simulation and the blue/lined histograms show the background distributions which are obtained from the data sidebands. Figure 4.9 shows the variables concerning the $\Delta \mathrm{R}$ separation as well as the distribution of the fit probability. The variables $\Delta R_{\max }(\mathrm{pK} \pi)$ and $\Delta \mathrm{R}_{\max }(\mathrm{l}-\mathrm{pK} \pi)$ seem the most promising and, as expected, they take in general slightly lower values for the signal case. The kinematic variables are shown in figure 4.10 and to a part in figure 4.11. The transverse momentum of the proton, the 
kaon and the $\Lambda_{c}$ as well as the ratio of the transverse momenta of the proton and the pion seem most promising. The momentum ratio $\mathrm{p}_{\mathrm{T}} \mathrm{p} / \mathrm{p}_{\mathrm{T}} \pi$ is higher in the signal case, which is expected owing to the proton mass being larger than the pion mass. This variable is shown in the range from zero to ten, as well as in the enlarged range around one. The invariant mass of the lepton- $\Lambda_{c}$ combination lies for the signal events as expected between the masses of the $\Lambda_{c}$ and the $\Lambda_{b}$. The variables concerning lifetime and decay length measurements are shown in figure 4.11. The variables $\mathrm{L}_{\mathrm{xy}}$ (p.v. $\left.-\Lambda_{\mathrm{c}}\right)$ and $c \tau\left(\Lambda_{c}\right)$ appear the most promising of all mentioned variables. The variables $\mathrm{dL}_{\mathrm{xy}}\left(\Lambda_{\mathrm{b}}\right), \mathrm{dL}_{\mathrm{xy}}\left(\Lambda_{\mathrm{c}}\right)$ are not used because of their discriminating power (which is low), but rather to ensure good quality in the vertex reconstruction.

To take into account correlations between the above variables, the first optimisation was done by scanning up to six variables simultaneously for the cuts that yield the best $s / \sqrt{b}$ ratio. The number of signal events $s$, that remain after application of the respective cuts, is obtained from the realistic simulation. The number of background events $b$ is determined from data, using the $3 \sigma$-wide sidebands in the $p K \pi$ invariant mass plot shown in figure 4.8 (b). In a second step, variables where a cut could change the shape of the lifetime distribution of the $\Lambda_{b}$ baryon, c $\tau\left(\Lambda_{b}\right)$, were considered individually, as placing a cut on those variables requires more care. Owing to the correlations amongst variables, it turns out that not all variables with seemingly good discriminating power are useful for the signal selection. Figures 4.12-4.14 show all variables again, this time after application of all selection cuts but the one chosen for the variable depicted in each case. The value of the selection cut for the respective variable is indicated by the vertical line with an arrow pointing towards the accepted area. If there is no line, no cut is used for the variable in question. The reasoning behind the selected choice of cut values is explained below.

No cut is used for the $\Delta \mathrm{R}$ variables, apart from a standard cut on $\Delta \mathrm{R}_{\max }(\mathrm{l}-\mathrm{pK} \pi)$ at 1.2 to ensure that the $\Lambda_{c}$ candidate tracks are in the vicinity of the lepton. A comparison of figure 4.12 and figure 4.9 shows that the initially good discrimination power of the variables depicted here is negligible after the application of the cuts on the other variables. 

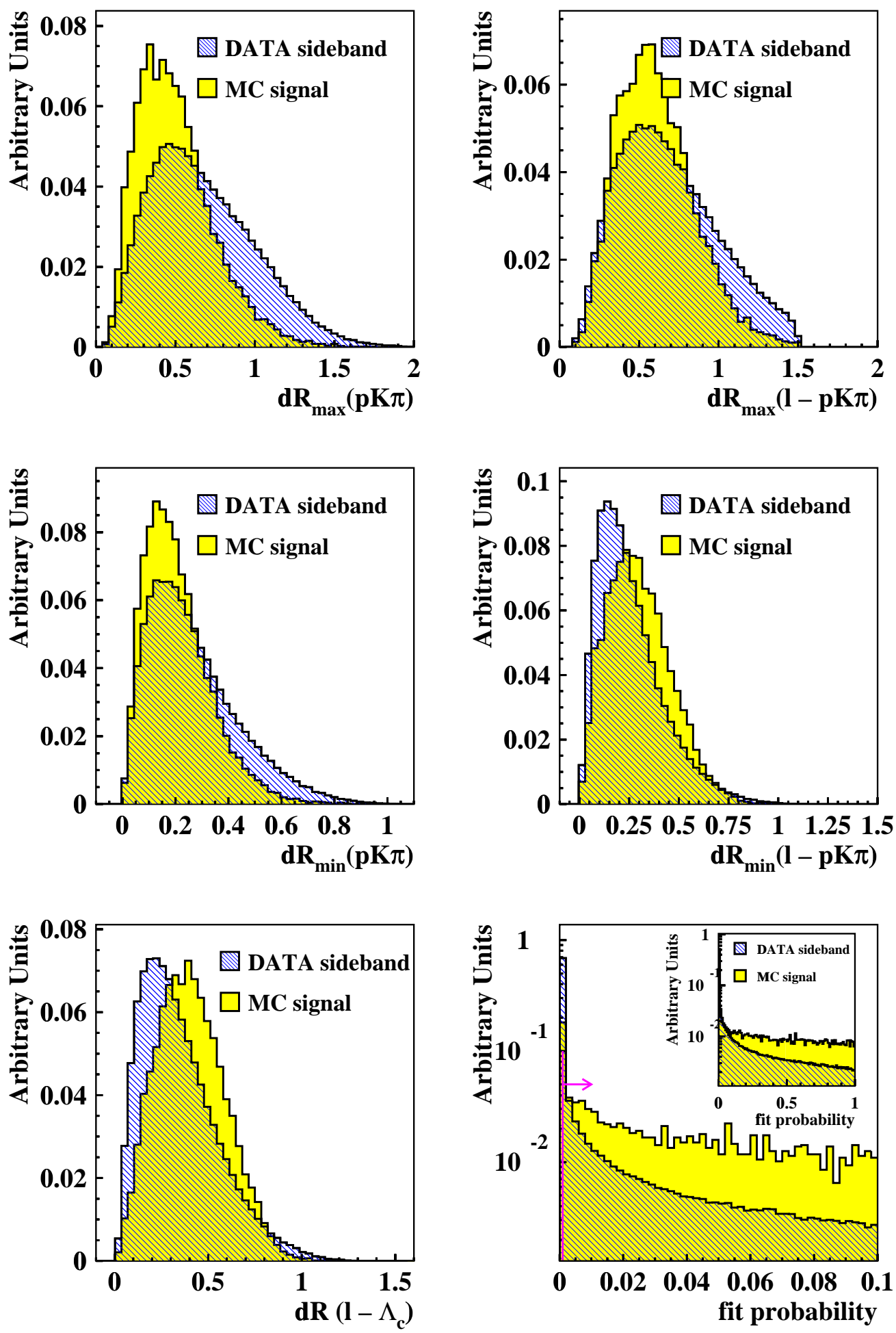

Figure 4.9: The first set of variables considered as cut variables in the signal selection. No selection cuts are applied at this stage. The vertex fit probability is required to be larger or equal to 0.001 . 

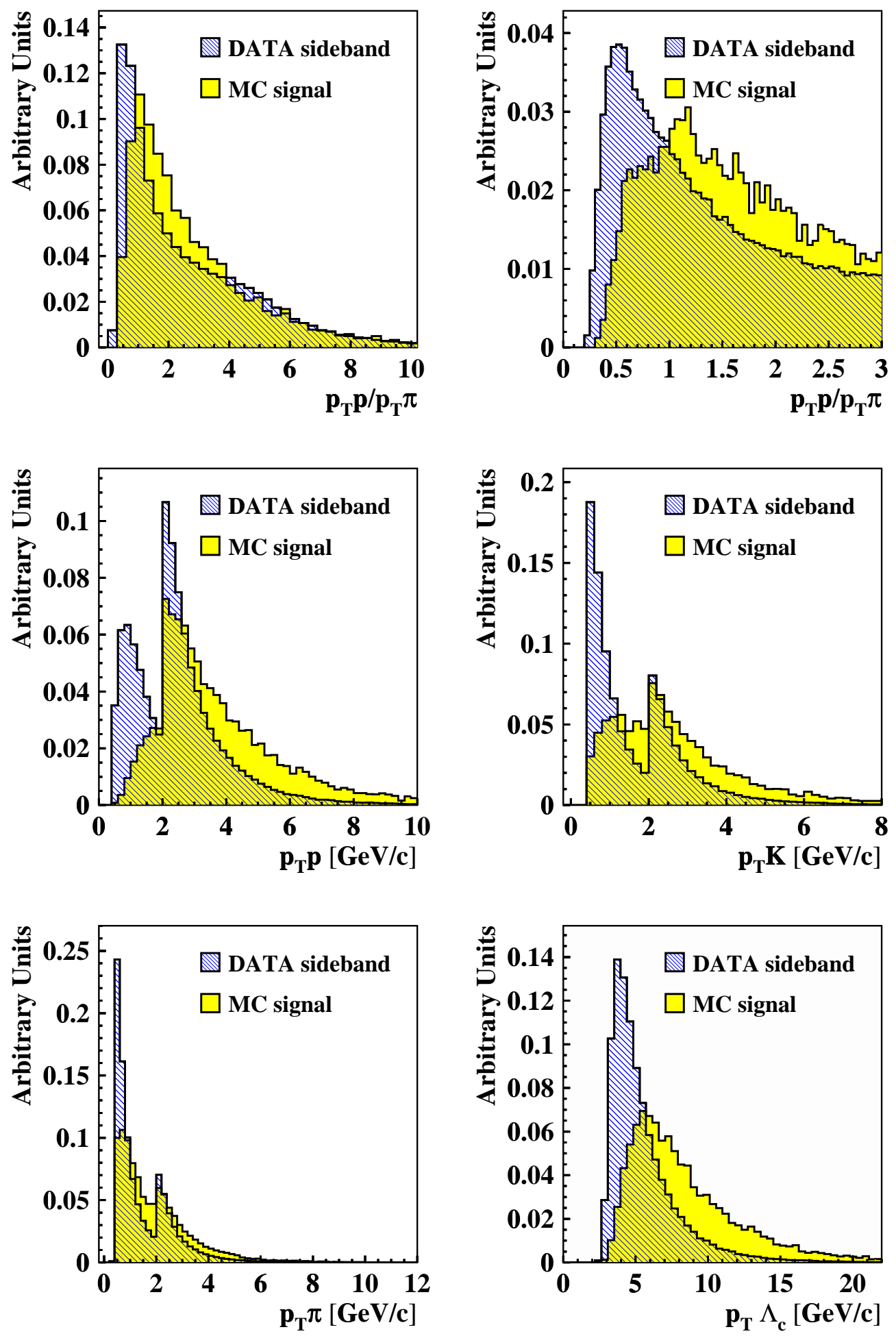

Figure 4.10: The second set of variables considered as cut variables in the signal selection. No selection cuts are applied at this stage. The vertex fit probability is required to be larger or equal to 0.001 . 

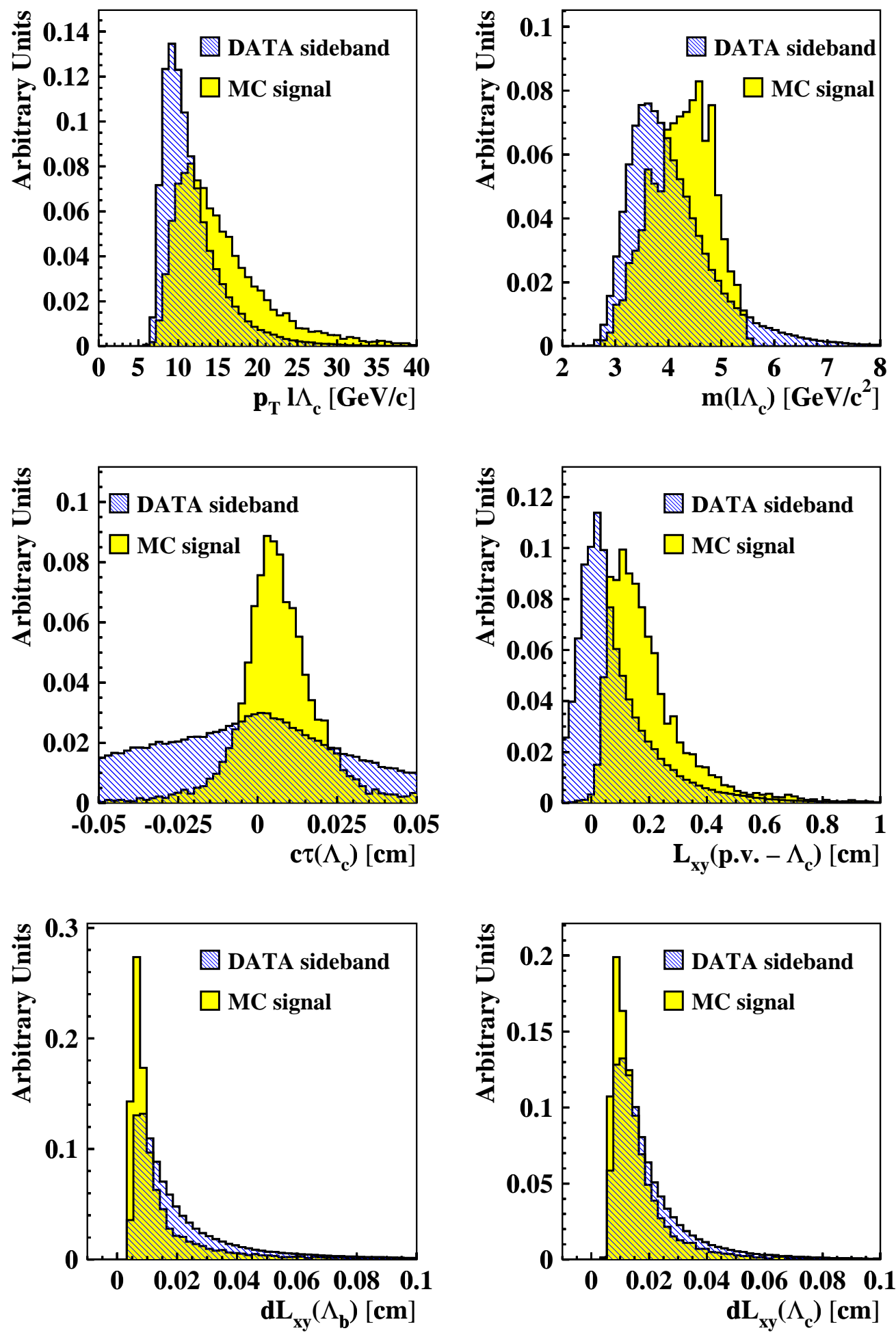

Figure 4.11: The third set of variables considered as cut variables in the signal selection. No selection cuts are applied at this stage. The vertex fit probability is required to be larger or equal to 0.001 . 

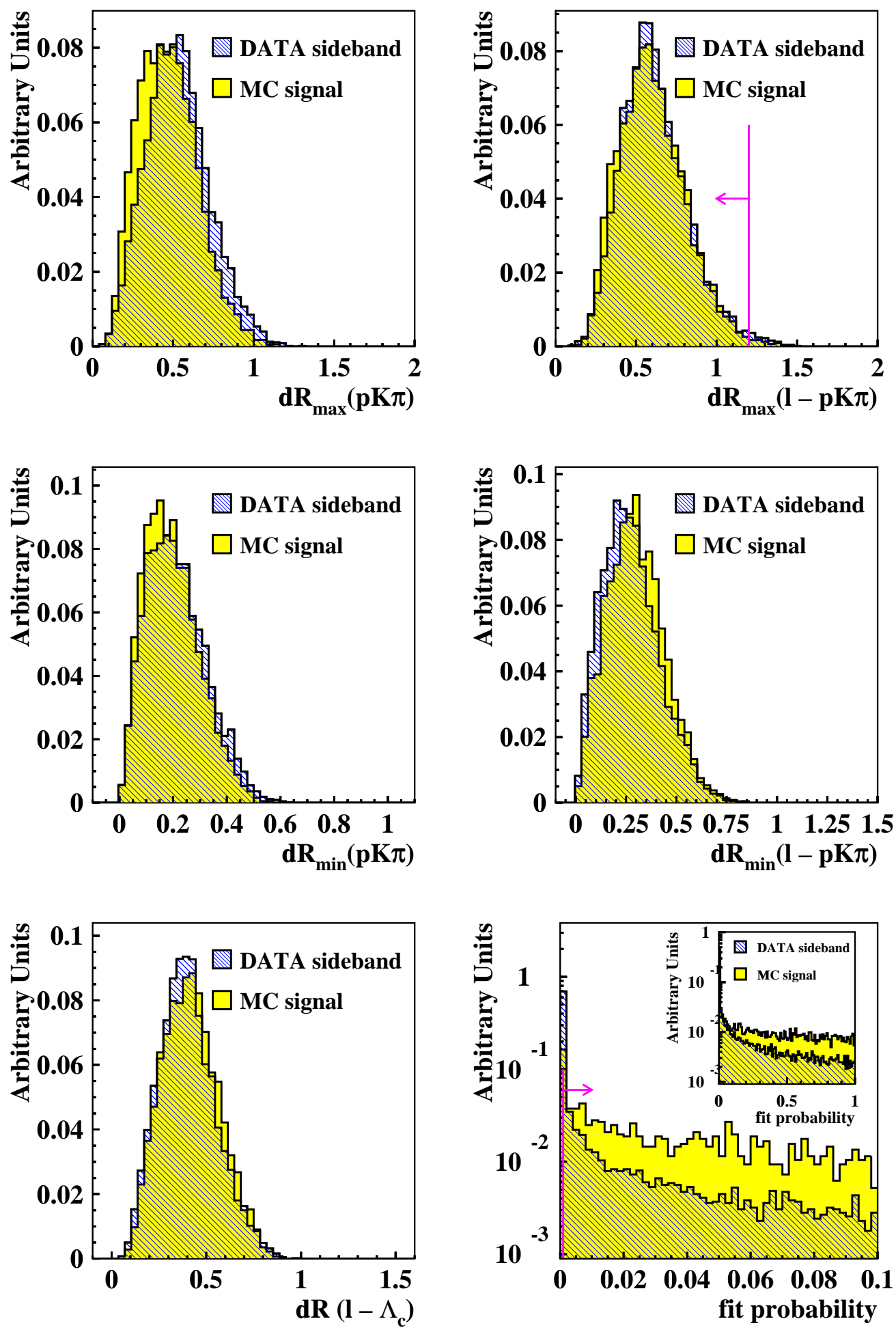

Figure 4.12: The first set of variables considered as cut variables in the signal selection. In each case all the selections cuts are applied apart from the cut on the variable itself. The cut value, if a cut is applied for the respective variable, is indicated by the vertical line with an arrow pointing towards the accepted region. 

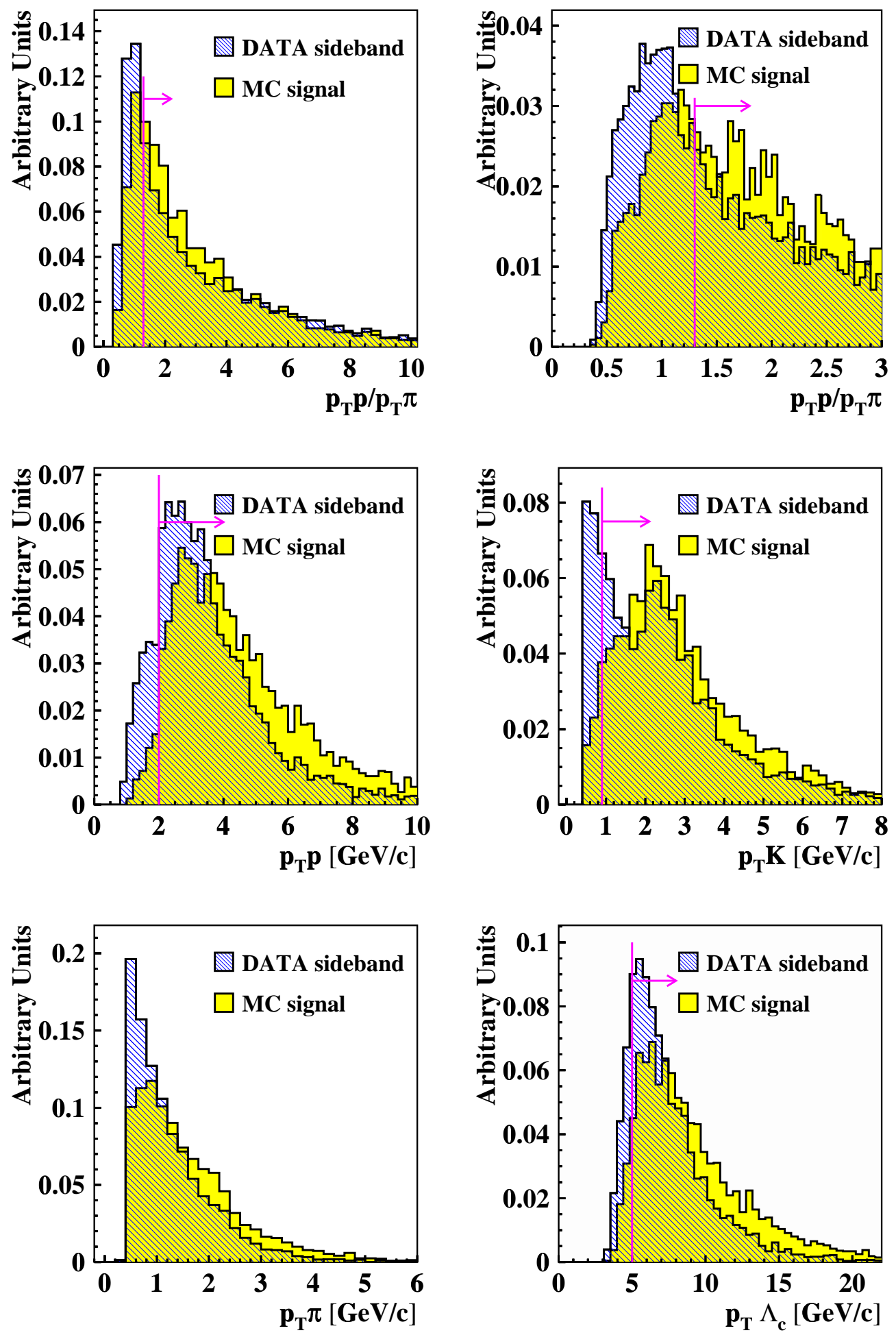

Figure 4.13: The second set of variables considered as cut variables in the signal selection. In each case all the selections cuts are applied apart from the cut on the variable itself. The cut value, if a cut is applied for the respective variable, is indicated by the vertical line with an arrow pointing towards the accepted region. 

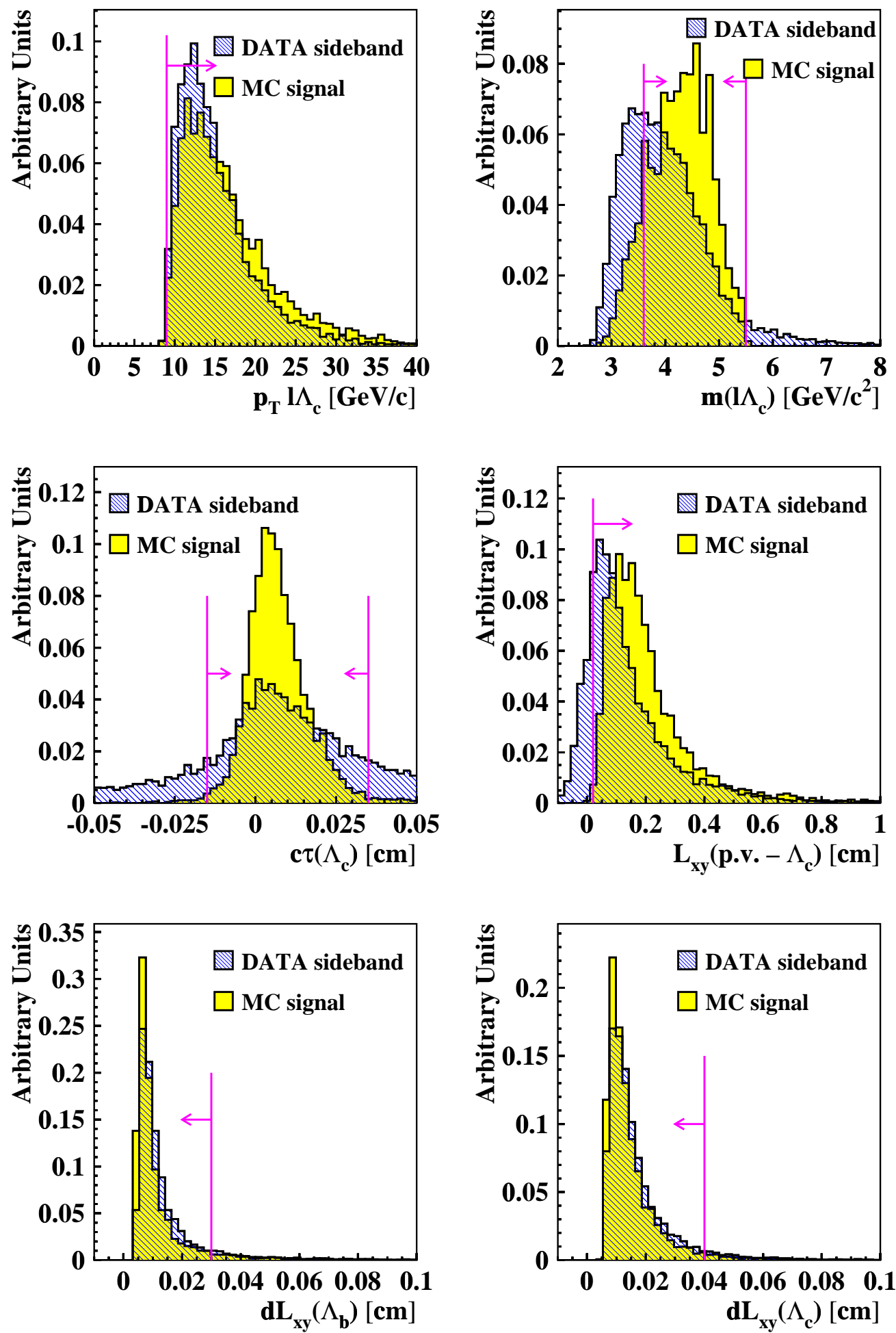

Figure 4.14: The third set of variables considered as cut variables in the signal selection. In each case all the selections cuts are applied apart from the cut on the variable itself which is indicated by the vertical line with an arrow pointing towards the accepted region. 
The momentum cuts $\mathrm{p}_{\mathrm{T}} \mathrm{p} \geq 2 \mathrm{GeV} / \mathrm{c}, \mathrm{p}_{\mathrm{T}} \mathrm{K} \geq 0.9 \mathrm{GeV} / \mathrm{c}$ and the cut on the invariant mass of the lepton- $\Lambda_{c}$ combination, $3.6 \mathrm{GeV} / \mathrm{c}^{2} \leq \mathrm{m}\left(1 \Lambda_{\mathrm{c}}\right) \leq 5.5 \mathrm{GeV} / \mathrm{c}^{2}$ are result of the multidimensional scan.

For the transverse momentum of the $\Lambda_{c}$ the scan returned a suggested cut value of $4.3 \mathrm{GeV} / \mathrm{c}$. However this cut is increased to $5 \mathrm{GeV} / \mathrm{c}$ to reject backgrounds from true $\Lambda_{c}$ candidates that do not originate from the signal decay of a $\Lambda_{b}$ baryon (see section 4.5). The cut on the transverse momentum of the lepton- $\Lambda_{c}$ combination is set to $9 \mathrm{GeV} / \mathrm{c}$ to further reject backgrounds from true physics processes. The cut value for $\mathrm{p}_{\mathrm{T}} \mathrm{p} / \mathrm{p}_{\mathrm{T}} \pi$ at 1.3 rejects backgrounds from other charm baryons and reflections from the $\Lambda_{c}$ baryon. This is discussed in more detail in section 4.5.1.

The cuts on $\mathrm{dL}_{\mathrm{xy}}\left(\Lambda_{\mathrm{b}}\right)$ and $\mathrm{dL}_{\mathrm{xy}}\left(\Lambda_{\mathrm{c}}\right)$ at $0.03 \mathrm{~cm}$ and $0.04 \mathrm{~cm}$ respectively, ensure good quality for the measurement of the decay length, so that they should be referred to as quality cuts rather than selection cuts.

Determining a cut value for $c \tau\left(\Lambda_{c}\right)$ and $\mathrm{L}_{\mathrm{xy}}\left(\right.$ p.v. $\left.-\Lambda_{\mathrm{c}}\right)$ requires some care, as these variables are correlated with the $c \tau$ value of the $\Lambda_{b}$ baryon. The cut values here were found by testing several possible cut values for their background rejection while ensuring that the cut does not significantly impact the $\mathrm{c} \tau\left(\Lambda_{b}\right)$ distribution. It is expected that the higher the cut on $\mathrm{L}_{\mathrm{xy}}\left(\mathrm{p} . \mathrm{v} .-\Lambda_{\mathrm{c}}\right)$, the more the $\mathrm{c} \tau\left(\Lambda_{b}\right)$ distribution is shifted to higher values. The multidimensional scan would have suggested a cut value of $0.06 \mathrm{~cm}$ for $\mathrm{L}_{\mathrm{xy}}\left(\mathrm{p} . \mathrm{v} .-\Lambda_{\mathrm{c}}\right)$, as well as $0.008 \mathrm{~cm} \leq \mathrm{c} \tau\left(\Lambda_{\mathrm{c}}\right) \leq 0.022 \mathrm{~cm}$. However figure 4.15 (a) shows that already a cut $\mathrm{L}_{\mathrm{xy}}\left(\mathrm{p} . \mathrm{v} .-\Lambda_{\mathrm{c}}\right) \geq 0.05 \mathrm{~cm}$ would bias the lifetime distribution of the $\Lambda_{b}$ baryon. Looking at the $\mathrm{c} \tau\left(\Lambda_{b}\right)$ distribution for various different cuts, the following values were chosen: $L_{x y}\left(\right.$ p.v. $\left.-\Lambda_{c}\right) \geq 0.02 \mathrm{~cm}$ and $-0.015 \mathrm{~cm} \leq \mathrm{c} \tau\left(\Lambda_{\mathrm{c}}\right) \leq 0.035 \mathrm{~cm}$. Figure $4.15(\mathrm{~b})$ shows the $\mathrm{c} \tau\left(\Lambda_{b}\right)$ distribution before and after the cut on $\mathrm{L}_{\mathrm{xy}}\left(\mathrm{p} . \mathrm{v} .-\Lambda_{\mathrm{c}}\right)$ at $0.02 \mathrm{~cm}$, figure 4.15 (c) shows the same distribution before and after the $\mathrm{c} \tau\left(\Lambda_{c}\right)$ cut. Figure 4.15 (d) finally shows the distribution before and after the application of both cuts. It can be seen in those figures that the chosen cuts do not significantly affect the $c \tau\left(\Lambda_{b}\right)$ distribution.

In addition to the variables described above there are a few quantities not simulated in the $\mathrm{MC}$ that add discriminating power between signal and background events. Figure 4.16 (a) shows the $p K \pi$ invariant mass peak after the application of all previously mentioned selection cuts. Now that a clean signal is visible, the 


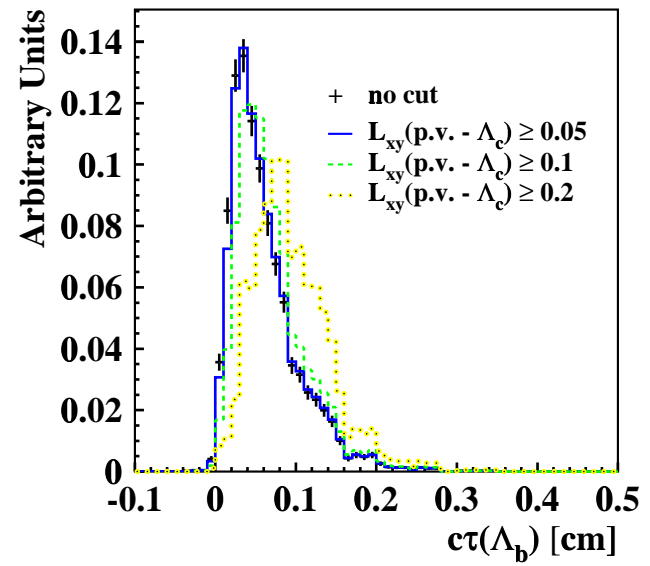

(a)

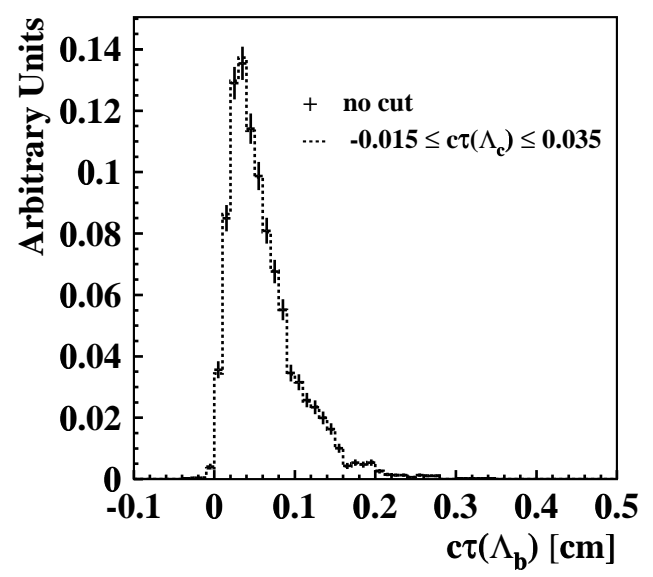

(c)

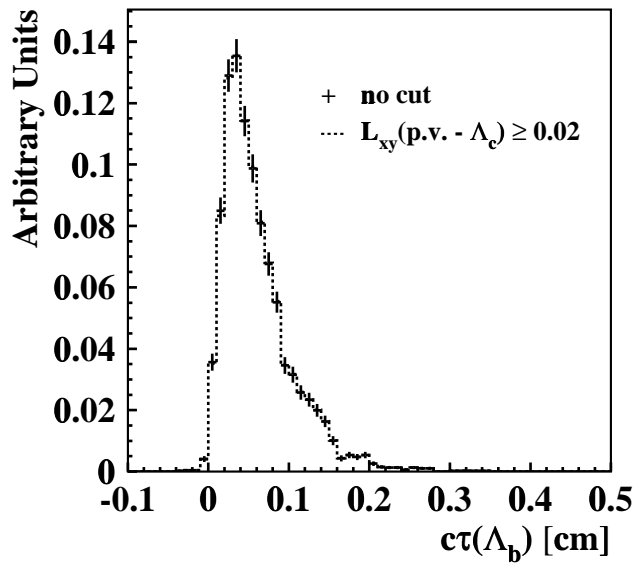

(b)

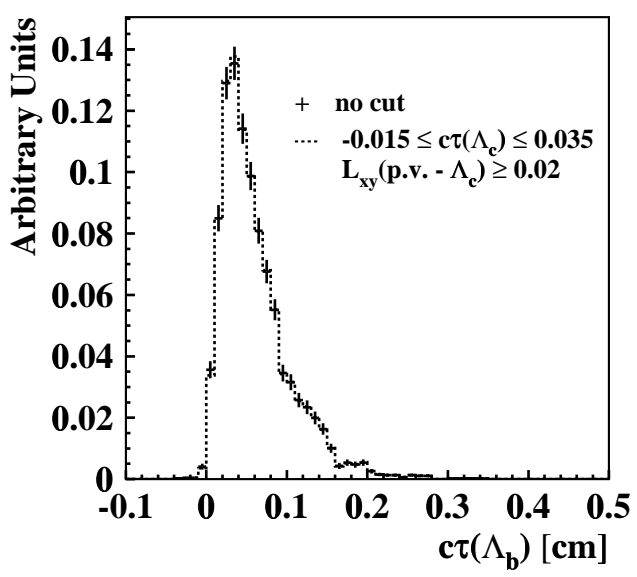

(d)

Figure 4.15: The $c \tau\left(\Lambda_{b}\right)$ distributions for various different cut values on $\mathrm{L}_{\mathrm{xy}}$ (p.v. $-\Lambda_{\mathrm{c}}$ ) and $c \tau\left(\Lambda_{c}\right)$. The respective cut values are indicated in the figure.

data itself are used to determine safe cuts for additional variables, i.e. cuts that do not reject a significant amount of true signal events. This is done by looking at the distribution under consideration for events from the $p K \pi$ invariant mass sidebands and comparing it to the same distribution obtained from events that fall into an area of $3 \sigma$ to either side of the mean value of the $\Lambda_{c}$ peak. The histograms for the signal region and the background region are then plotted on top of each other. This shows regions of excess events which, assuming that the background under the signal peak is distributed like the background from the sidebands for the variable in question, are assumed to be from the signal. Subtracting the two histograms yields the signal distribution. 


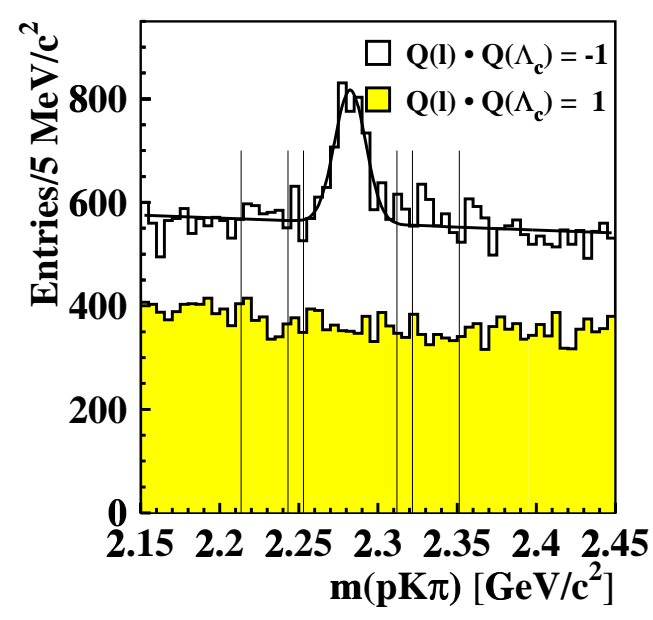

(a)

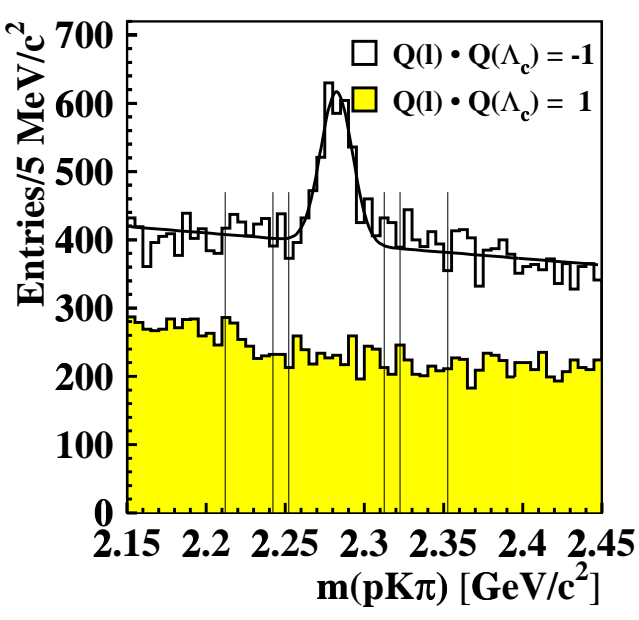

(b)

Figure 4.16: The figures (a) and (b) show the $p K \pi$ invariant mass distribution before and after the application of the cut on the ptratio variables respectively.

One of the variables only available in data is the excess energy or momentum in a $\Delta \mathrm{R}$ cone around the lepton or the lepton- $\Lambda_{c}$ pair. Excess momentum refers here to the momentum present additionally to that of the $\Lambda_{b}$ candidate tracks. In the MC only the $\Lambda_{b}$ decay is simulated. Thus there are no excess tracks apart from a few additional photons and $e^{+} e^{-}$pairs created during simulation of the particle's path through the detector material.

In the data however there exist additional tracks unrelated to the $\Lambda_{b}$ decay, so that a cut on variables of this kind is determined from the data itself. Two variables are defined:

$$
\text { ptratio1 }=\frac{\mathrm{p}_{\mathrm{T}} \mathrm{p}+\mathrm{p}_{\mathrm{T}} \mathrm{K}+\mathrm{p}_{\mathrm{T}} \pi+\mathrm{p}_{\mathrm{T}} \mathrm{l}}{\mathrm{p}_{\text {tot }}}
$$

and

$$
\text { ptratio } 2=\frac{\mathrm{p}_{\mathrm{T}} \mathrm{p}+\mathrm{p}_{\mathrm{T}} \mathrm{K}+\mathrm{p}_{\mathrm{T}} \pi+\mathrm{p}_{\mathrm{T}} \mathrm{l}}{\mathrm{p}_{\text {totl }}} ;
$$

$\mathrm{p}_{\text {tot }}$ and $\mathrm{p}_{\text {totl }}$ are the sum of the transverse momenta of all tracks that lie in a $\Delta \mathrm{R} \leq 1$ cone around the resulting transverse momentum of lepton- $\Lambda_{c}$ and the lepton itself respectively. Figure 4.17 (a) and (b) show the two variables for events whose $p K \pi$ invariant mass falls into the signal region (white histogram) and the background region (shaded histogram). Figure 4.17 (c) shows the variable ptratio2 


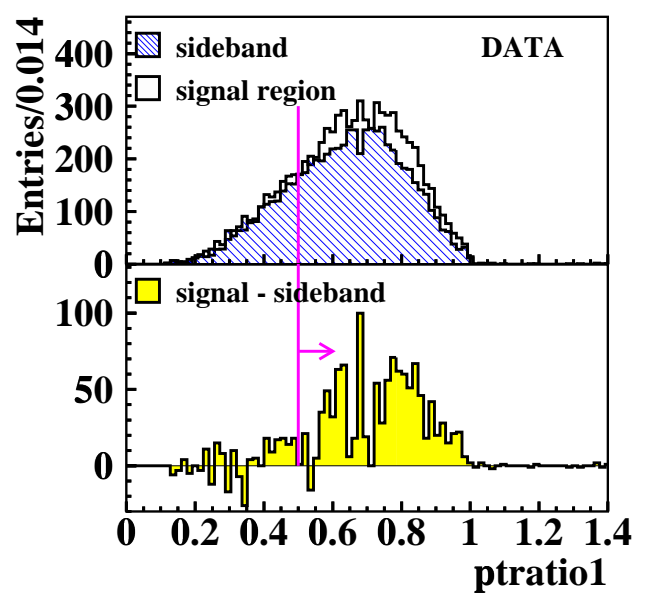

(a)

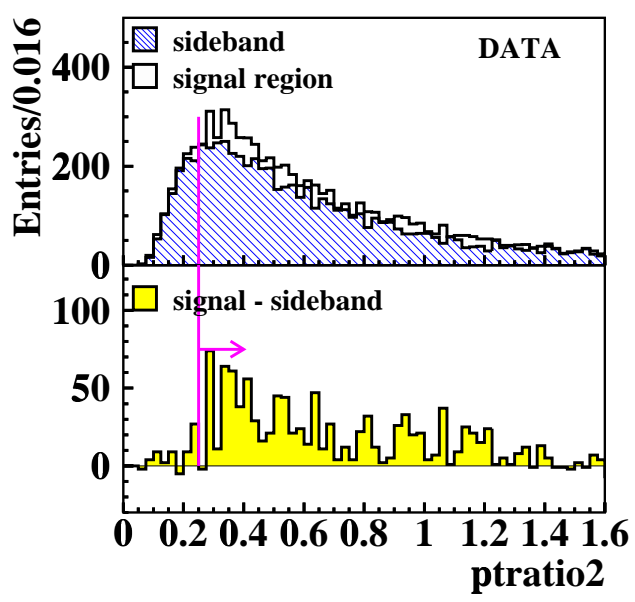

(b)

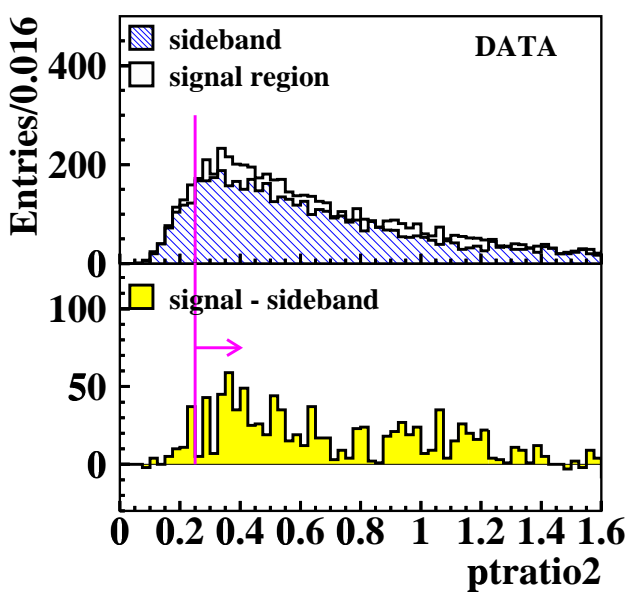

(c)

Figure 4.17: Figures (a) and (b) show the variables ptratio1 and ptratio2 for data. Each figure is divided into two halfs. In the upper half, the white histogram shows events that fall into the signal region of the $p K \pi$ invariant mass plot and the shaded histogram shows events that fall into the sideband region. The histogram in the lower half shows the difference between the two histograms from the upper half, yielding the sideband-subtracted signal distribution. Figure (c) shows the variable ptratio2 after application of the cut ptratio $1 \geq 0.5$.

after application of the cut ptratio $1 \geq 0.5$. In all three figures, the lower plot shows the difference of the two histograms of the upper plot. The vertical line indicates the selected cut value with the arrow again pointing towards the region of selected events. Figure 4.16 (b) shows the $p K \pi$ invariant mass distribution after the application of these two cuts. 
The last set of variables not simulated in the MC concerns the $\mathrm{dE} / \mathrm{dx}$ measurement of a track. As described in section 4.2.2, the COT provides some discrimination power between particles of different species via a measurement of their specific energy loss. A dE/dx measurement can help to distinguish protons from kaons and pions and can thus enhance the fraction of correctly identified protons. From the universal curve measured for the COT $[45,46]$ it is possible to predict a $\mathrm{dE} / \mathrm{dx}$ value for a given velocity of the particle, i.e. for a given momentum and a given mass hypothesis. The $\mathrm{dE} / \mathrm{dx}$ variables considered here are:

$$
\begin{aligned}
\operatorname{dedxp} & =\frac{\mathrm{dE} / \mathrm{dx}_{\text {meas. }}(\mathrm{p})-\mathrm{dE} / \mathrm{dx}_{\text {pred }}(\mathrm{p})}{\sigma(\mathrm{p})} \\
\operatorname{dedxk} & =\frac{\mathrm{dE} / \mathrm{dx}_{\text {meas. }}(\mathrm{p})-\mathrm{dE} / \mathrm{dx}_{\text {pred }}(\mathrm{K})}{\sigma(\mathrm{p})} \\
\operatorname{dedxpi} & =\frac{\mathrm{dE} / \mathrm{dx}_{\text {meas. }}(\mathrm{p})-\mathrm{dE} / \mathrm{dx}_{\text {pred }}(\pi)}{\sigma(\mathrm{p})}
\end{aligned}
$$

The variables $\mathrm{dE} / \mathrm{dx}_{\text {pred }}(\mathrm{p}), \mathrm{dE} / \mathrm{dx}_{\text {pred }}(\mathrm{K})$ and $\mathrm{dE} / \mathrm{dx}_{\text {pred }}(\pi)$ are the $\mathrm{dE} / \mathrm{dx}$ values predicted by inserting the momentum of the proton track together with a mass hypothesis $\left(\mathrm{m}=\mathrm{m}_{\mathrm{p}}, \mathrm{m}_{\mathrm{K}}\right.$ and $\mathrm{m}_{\pi}$ respectively) into the universal curve; $\sigma(\mathrm{p})$ is the error on the $\mathrm{dE} / \mathrm{dx}$ measurement for the proton track.

Figure 4.18 (a) shows the distribution of dedxp together with the cut value chosen for it. The cut is rather loose to make this a safe cut, i.e. to avoid cutting out real signal events. The three variables dedxp, dedxk, and dedxpi are correlated, which can be seen in figure 4.18 (b). Indeed most of the discriminating power of the variables dedxk and dedxpi is lost after the application of the cut on dedxp. The distributions in the middle of figure 4.18 show the variable dedxk before (c) and after (d) the dedxp cut. The histograms in the bottom of figure 4.18 show the same for the variable dedxpi. The cut values indicated have little effect after the cut on dedxp.

Figure 4.19 (a) shows the $p K \pi$ invariant mass plot after all cuts using good runs. Figure 4.19 (b) shows the mass plot before the cut on pratio1, pratio2 and the dedx variables (histogram 1), after the cut on pratio1 and pratio2 (histogram 2) and again after all cuts (histogram 3). 

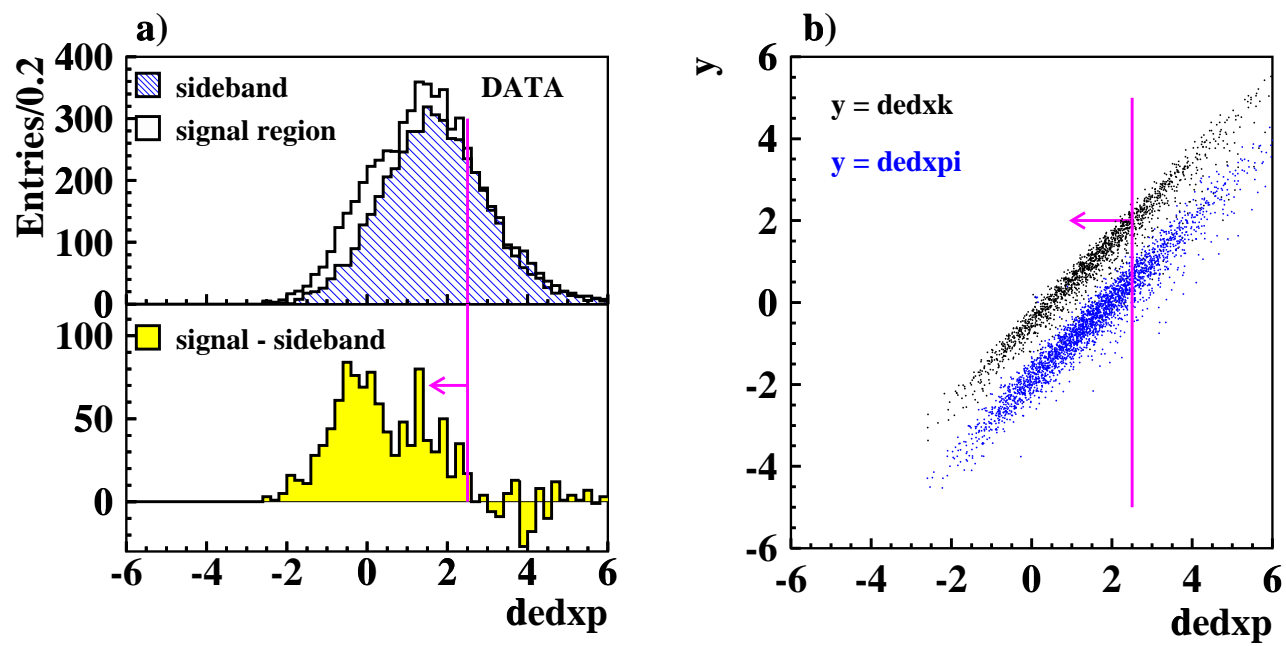

c)

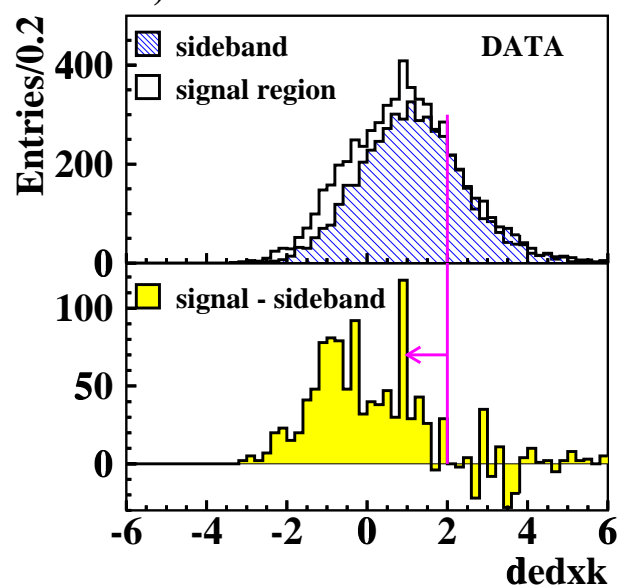

d)

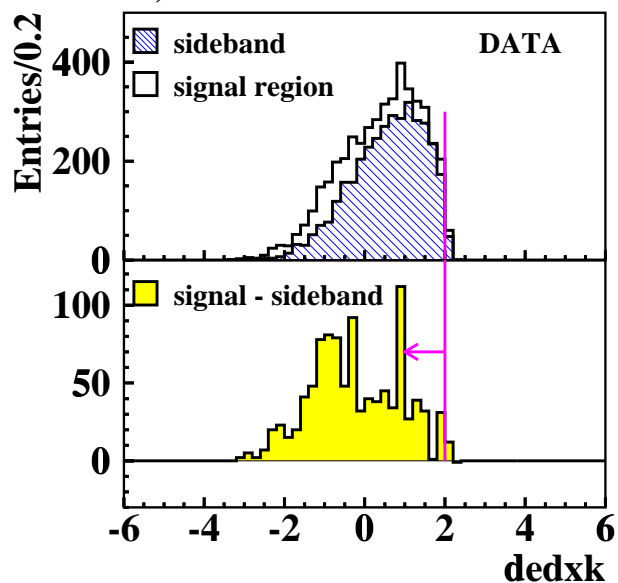

e)
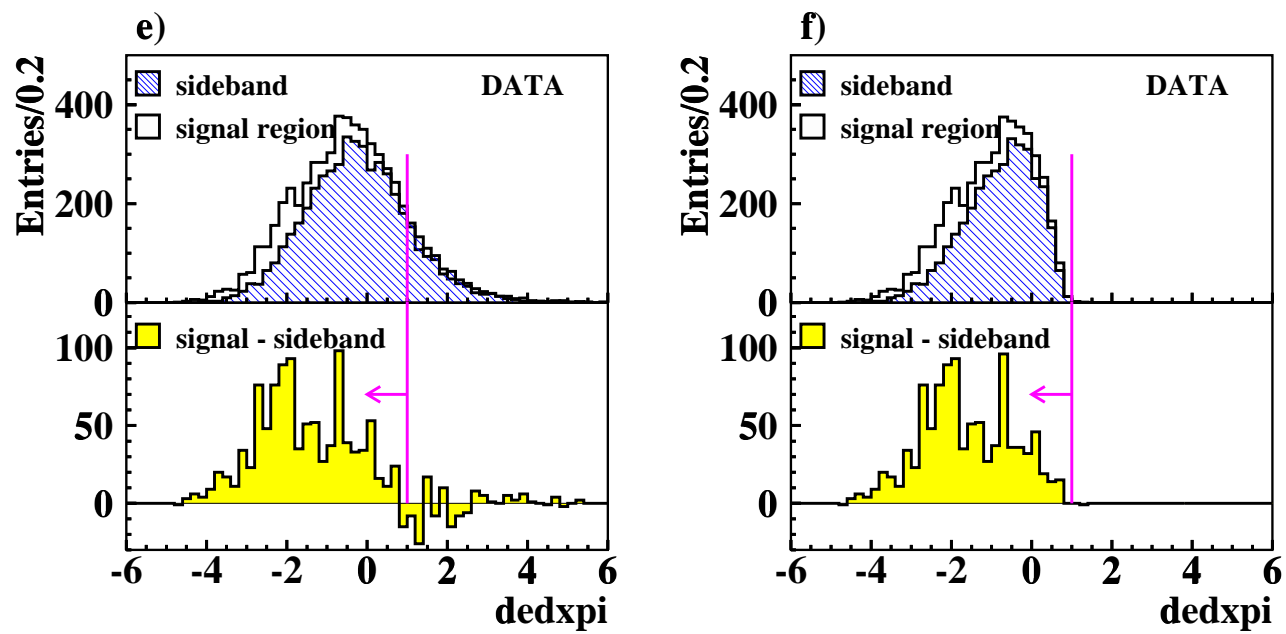

Figure 4.18: The $\mathrm{dE} / \mathrm{dx}$ distributions obtained from data. The yellow(grey) histogram is obtained by subtracting the histogram for the sideband region (shaded) from the histogram for the signal region (white). Figure (a) shows dedxp and figure (b) shows the correlations between dedxp-dedxk (dots on upper diagonal) and dedxp-dedxpi (dots on lower diagonal). Figures (c) and (e) show dedxk and dedxpi before the application of the cut on dedxp and figures (d) and (f) show the same distributions after its application. 


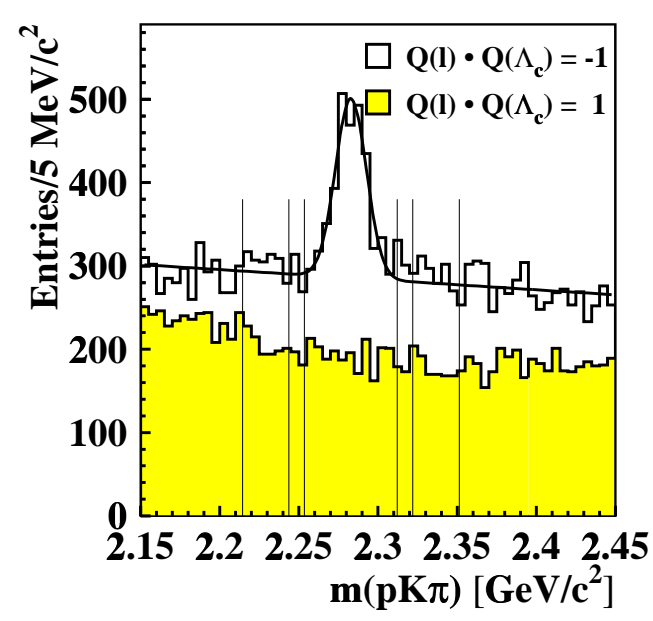

(a)

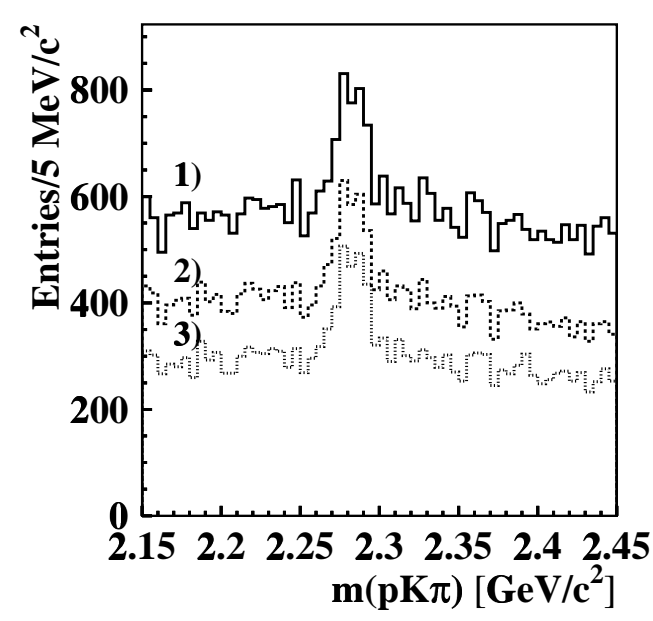

(b)

Figure 4.19: Figure (a) shows the $p K \pi$ invariant mass distribution after application of all selection cuts. In figure (b) the mass plot can be seen before the cut on pratio1, pratio2 and the dedx variables (histogram 1), after the cut on pratio1 and pratio2 (histogram 2) and again after all cuts (histogram 3).

For the sake of completeness the cut variables are shown again in figures 4.20-4.22 after application of all cuts using the data in the representation of figures 4.17 and 4.18. The cuts originally chosen by using $\mathrm{MC}$ for the signal distributions and the data sidebands for the background distributions are again indicated by the vertical line. This can be seen as a cross-check for the credibility of the cuts and furthermore indicates that there is a reasonable agreement between data and MC.

Appendix A shows a direct comparison of data and MC distributions as well as a comparison of the realistic simulation and the parametric simulation. The agreement between data and $\mathrm{MC}$ is reasonable; however, due to the large background under the $\Lambda_{c}$ signal, the errors and fluctuations in the data distributions are large. Data-MC comparisons were also performed using a control sample of $\mu+D_{0}$ events [51]. The MC used there was produced with the same methods as in this analysis and the agreement shown is good.

The selection cuts described above mainly reject combinatorial background, although some, especially the cut on the invariant mass of the lepton- $\Lambda_{c}$ combination, also reject backgrounds in which the three candidate tracks originate from the decay of other charm hadrons. The next section studies those backgrounds. 

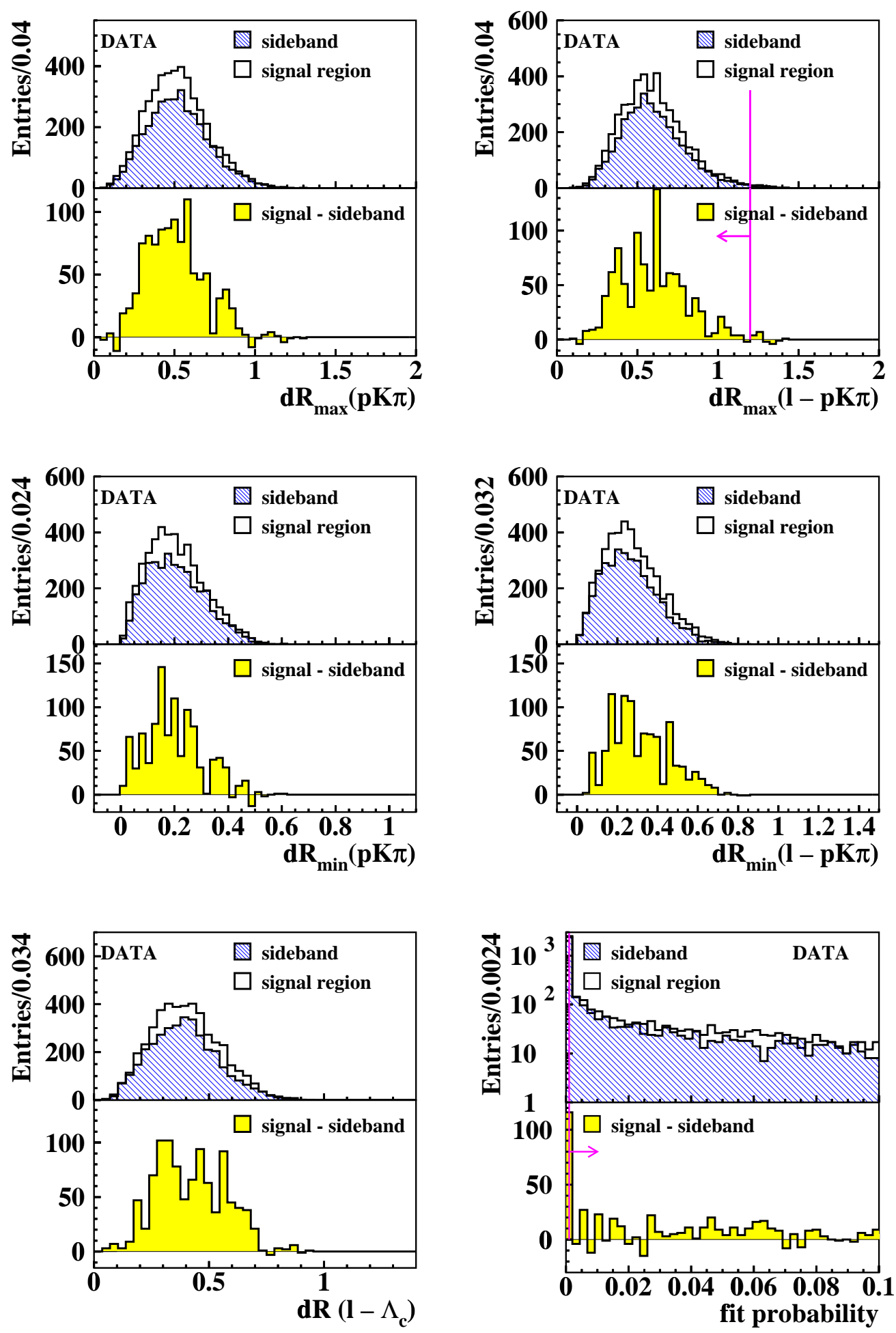

Figure 4.20: The first set of variables considered as cut variables in the signal selection. All selections cuts, including cuts on $\mathrm{dE} / \mathrm{dx}$ are applied, apart from the cut on the variable itself. The cut value, if a cut is applied for the respective variable, is indicated by the vertical/magenta line with an arrow pointing towards the accepted region. 

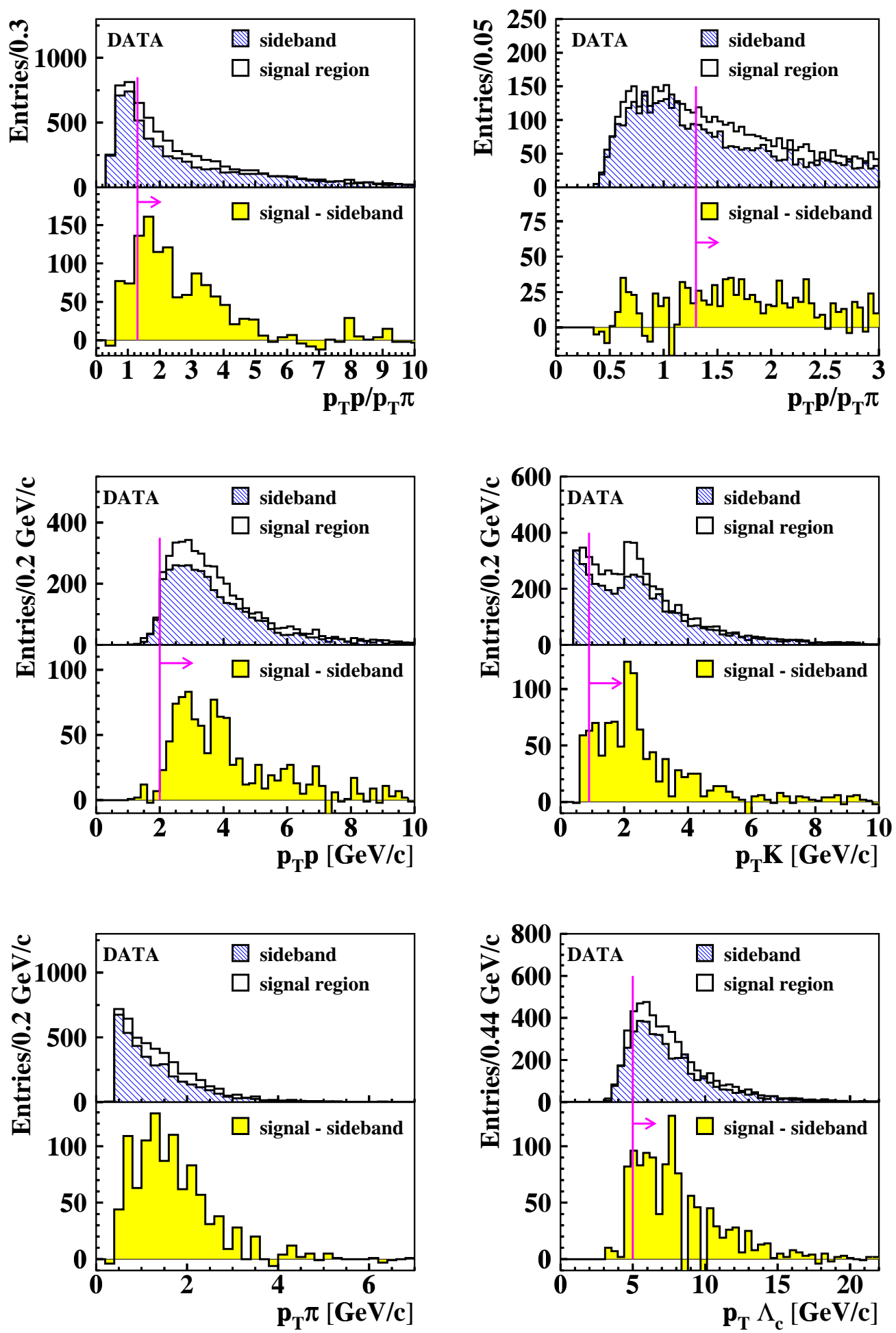

Figure 4.21: The second set of variables considered as cut variables in the signal selection. All selections cuts, including cuts on $\mathrm{dE} / \mathrm{dx}$ are applied, apart from the cut on the variable itself. The cut value, if a cut is applied for the respective variable, is indicated by the vertical/magenta line with an arrow pointing towards the accepted region. 

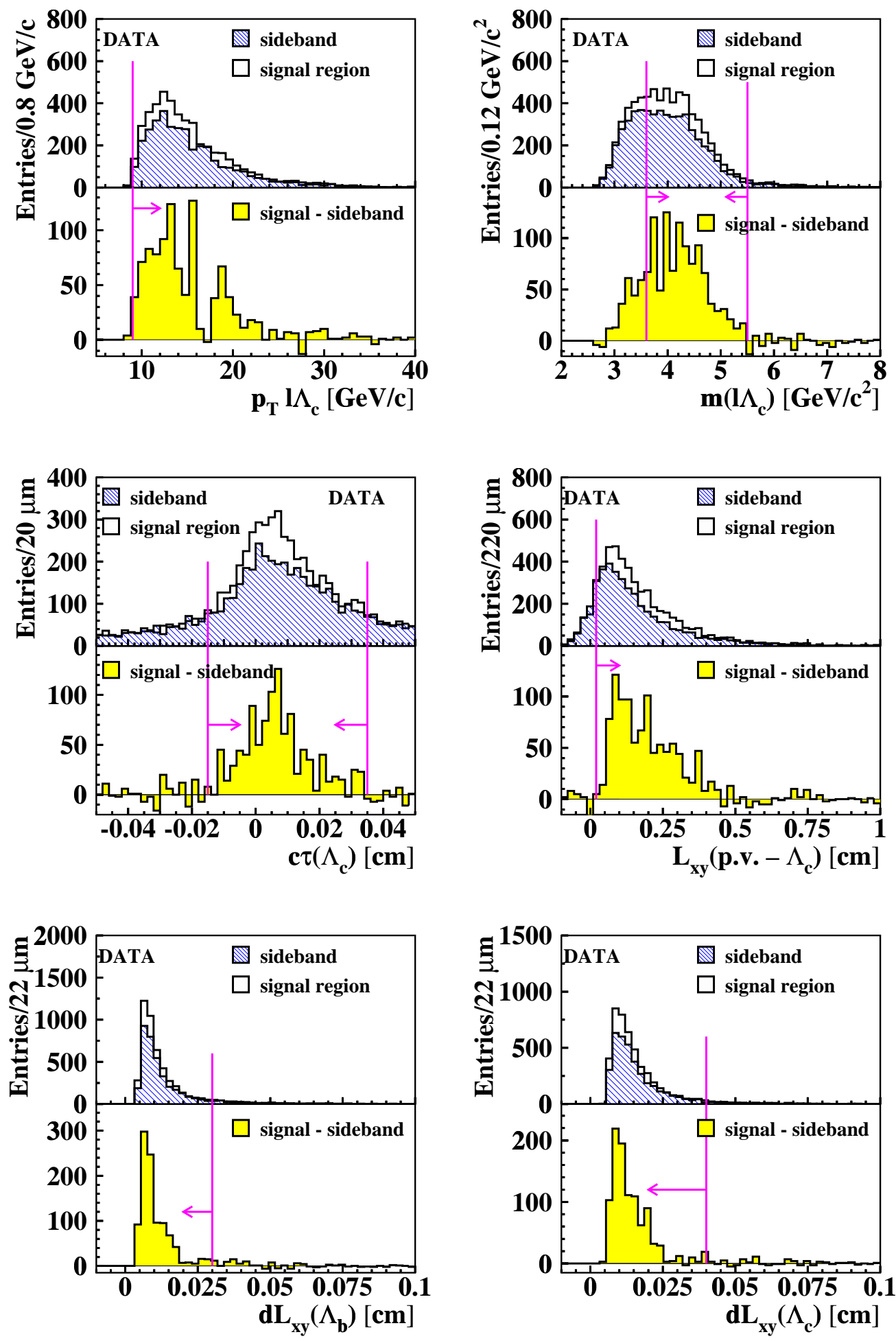

Figure 4.22: The third set of variables considered as cut variables in the signal selection. All selections cuts, including cuts on $\mathrm{dE} / \mathrm{dx}$ are applied, apart from the cut on the variable itself which is indicated by the vertical/magenta line with an arrow pointing towards the accepted region. 


\subsection{Background Processes}

The signature of the $\Lambda_{b}$ signal events is a lepton with opposite charge to a three track combination found in its vicinity. For true signal events these three tracks come from the decay $\Lambda_{c}^{+} \rightarrow p K^{-} \pi^{+}$; the two likesign tracks are the proton and the pion - their assignment is potentially ambiguous - and the oppositely charged track is a kaon. The $p K \pi$ invariant mass plot to the right shows that a significant amount of background survives the selection cuts. The various sources of background that can yield the same event signature, and the extent to which they can bias the $\Lambda_{b}$ lifetime measurement, are described in the follow-

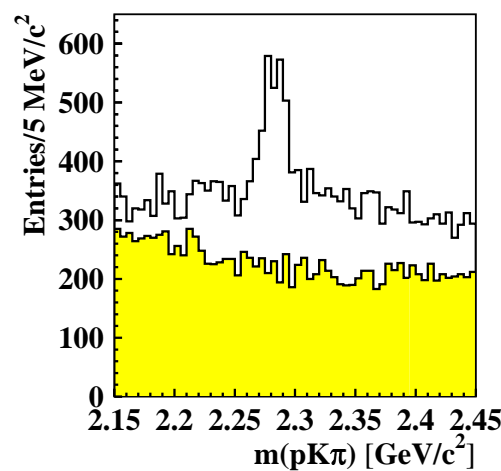

Figure 4.23: The figure shows the $p K \pi$ invariant mass plot for data (obtained using all runs) after selection cuts for the right-sign (white histogram) and the wrongsign (yellow/shaded histogram) combination. ing sections.

\subsubsection{Combinatorial Background and $\Lambda_{c}$ Reflections}

The most copiously produced background arises from tracks in the $\eta-\phi$ vicinity of a lepton that are randomly combined to form a $\Lambda_{c}$ baryon. This background however does not pose a serious problem for the lifetime measurement. The lepton, randomly associated with the three track combination, has no charge correlation with the latter, and the combinatorial background will thus equally contribute to right-sign and wrong-sign combinations. Much more importantly, the $p K \pi$ invariant mass distribution of the combinatorial background is not expected to peak around the invariant mass of the $\Lambda_{c}$. The wrong-sign combination shows indeed no peak in the signal region (see yellow/shaded histogram in the figure above).

The assignment of the proton and the pion mass to the like-sign tracks in a $\Lambda_{c}$ candidate is ambiguous. Figure 4.24 (a) shows the $p K \pi$ invariant mass distribution for events from the realistic MC simulation. The analysis code that is used to select $\Lambda_{c}$ candidates in the data is also applied to the MC. The white histogram shows the candidates with the correct mass assignment for proton and pion and the yellow/shaded histogram shows candidates with the wrong mass assignment, $\Lambda_{c}$ 


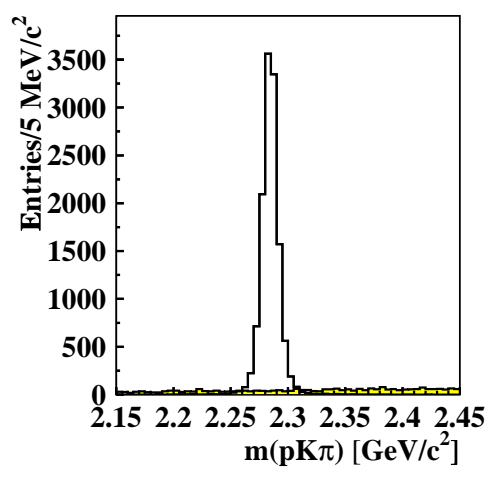

(a)

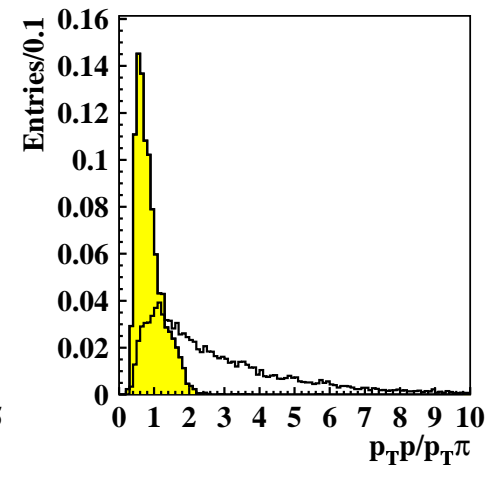

(b)

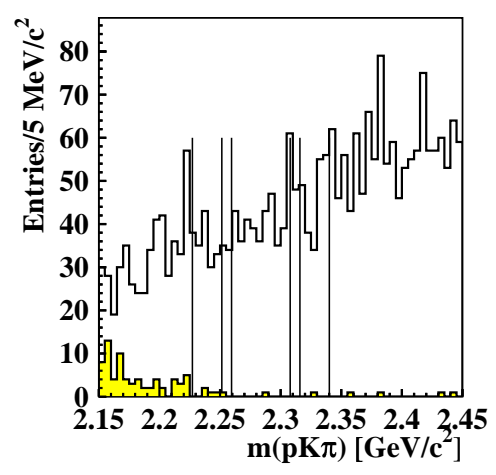

(c)

Figure 4.24: Figure (a) shows the $p K \pi$ invariant mass distribution for $\Lambda_{c}$ signal MC for the correct (white histogram) and the wrong (yellow/shaded) histogram) assignments of proton and pion mass. Figure (b) shows $\mathrm{p}_{\mathrm{T}} \mathrm{p} / \mathrm{p}_{\mathrm{T}} \pi$ for the correct and wrong mass assignments (white and yellow/shaded histogram respectively). Figure (c) shows again the $p K \pi$ invariant mass distribution for the wrong assignment (white histogram). The yellow/shaded histogram here contains only candidates with $\mathrm{p}_{\mathrm{T}} \mathrm{p} / \mathrm{p}_{\mathrm{T}} \pi>1.3$.

reflections, i.e. the true proton track is assigned the pion mass and vice versa. It can be seen that the distribution for the wrong mass assignment is very broad and only a small amount of the generated events populates the signal region. Without the application of selection cuts this distribution is flat and thus will not bias the lifetime measurement since the same number of events enters the fit via the sidebands and the signal region. However selection cuts, in particular a cut on the ratio of the transverse momenta of the proton and the pion, can change the shape of this curve thus introducing a bias.

Owing to the proton mass being larger than the pion mass, the $\mathrm{p}_{\mathrm{T}}$ of the proton from a $\Lambda_{c}$ decay is, without application of any selection cuts, in the majority of events (84\%) larger than the $\mathrm{p}_{\mathrm{T}}$ of the pion. A cut on the ratio of these two momenta is used in this analysis to reduce the combinatorial background and the background from real physics events from charm decays. Additionally this cut reduces the amount of $\Lambda_{c}$ reflections. Figure 4.24 (b) shows the distribution of $\mathrm{p}_{\mathrm{T}} \mathrm{p} / \mathrm{p}_{\mathrm{T}} \pi$ for candidates with the correct mass assignment (white histogram) and candidates with the wrong mass assignment (yellow/shaded histogram), which clearly shows that the $\mathrm{p}_{\mathrm{T}} \mathrm{p} / \mathrm{p}_{\mathrm{T}} \pi$ value for candidates with the wrong mass assignment peaks at values below one. 
Figure 4.24 (c) shows the $p K \pi$ invariant mass distribution for the wrong mass assignment. The white curve here is the yellow/shaded curve from figure 4.24 (a), the yellow/shaded curve contains only candidates with $\mathrm{p}_{\mathrm{T}} \mathrm{p} / \mathrm{p}_{\mathrm{T}} \pi \geq 1.3$. The cut is set at 1.3 to avoid the possibility that reflections, true $\Lambda_{c}$ candidates where the proton and the pion assignment are swapped, populate the signal and sideband region of the $\Lambda_{c}$ peak unevenly.

\subsubsection{Backgrounds from Real Charm Decays}

Various charm hadrons ultimately decay into three particles. These charm hadrons can come from semileptonic B decays and, even if they differ in their kinematics, can populate the invariant mass region around the $\Lambda_{c}$ mass. This can happen if their decay products are wrongly associated the proton, kaon and pion mass instead of their true particle masses. Considering that the production rates for $B^{0}$ and $B^{ \pm}$are approximately four times as high as that for $\Lambda_{b}$, while the rate for $B_{s}$ approximately equals the rate for $\Lambda_{b}$, this type of background can form a significant fraction of events in the signal and sideband region. If this background is not evenly distributed among signal and sidebands it can bias the lifetime measurement. The background from true charm decays and its reduction where viable is thus considered in the following section.

\section{Background from $D^{* \pm}(2010)$ and $D_{0}$}

$D^{*}(2010)^{ \pm}$mesons, in the following referred to as $D^{*}$ mesons, can populate the right-sign $p K \pi$ combination through $B^{0}$ decays: $B^{0} \rightarrow D^{*-} l^{+} \nu_{l}$. The $D^{*-}$ can decay into $\bar{D}^{0} \pi^{-}\left(67.7 \%\right.$ [1]) or $D^{-} \pi^{0}(30.7 \%$ [1]). It is the former decay that yields, after a decay of the $\bar{D}^{0}$ to $K^{+} \pi^{-}$, three charged particles in the final state that are in right-sign combination to the lepton from the $B_{0}$ decay:

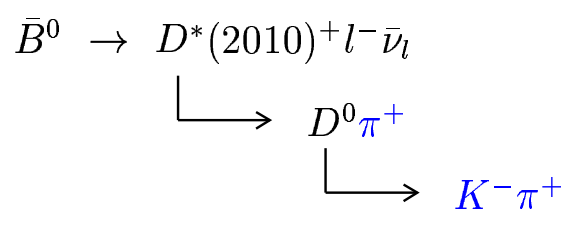


The like-sign particles here are two pions; the opposite sign track is, as in the case of $\Lambda_{c}$ decay, a kaon. The proton candidate among the $\Lambda_{c}$ candidate tracks could thus really be a pion from a $D^{*}$ decay.

The $D^{0}$ can also originate from the decay of a $B^{-}$:

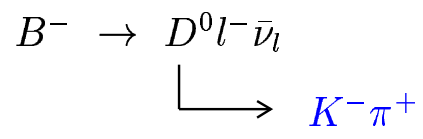

Although this decay yields only two tracks, the inclusion of a third random track from the event allows it to mimic a signal event. If two of the three candidate tracks form a $K \pi$ invariant mass with a value near the $D^{0}$ mass, they are most likely not part of a $\Lambda_{c}$ decay. The number of $D^{0}$ 's selected is expected to be larger than the number of $D^{*}$ s due to this additional source of $D^{0}$ mesons.

Figure 4.25 (a) shows the two track $K \pi$ invariant mass distribution for data, obtained by assigning the pion mass to the proton candidate and combining it with the kaon candidate. The solid and hashed histograms show the right and the wrong sign combination with the lepton respectively. The $D^{0}$ signal peak is not observed in the wrong sign combination. Forming the two track invariant mass with the original kaon and pion candidate tracks themselves yields no events around the $D^{0}$ mass. Figure 4.25 (b) shows the $p K \pi$ invariant mass plot using only events from the $D^{0}$ peak $\left(1.8218 \mathrm{GeV} / \mathrm{c}^{2} \leq \mathrm{m}(\mathrm{K} \pi) \leq 1.9012 \mathrm{GeV} / \mathrm{c}^{2}\right)$ together with the lines indicating the signal and the sideband region of the $\Lambda_{c}$ peak. The number of events in the signal region is approximately a factor of 1.4 higher than the number of events in the background region; as such it is desirable to cut out events from $D^{0}$ decays if the impact of such a cut on true $\Lambda_{c}$ signal events is negligible. Figure 4.25 (c) shows the $K \pi$ invariant mass distribution for $\Lambda_{c}$ signal MC. The majority of signal events ( $>99 \%$ ) lie below the $D^{0}$ mass region thus allowing the cut around the $D^{0}$ mass window to be applied. This cut will also automatically remove any $D^{*}$ candidates that might be present.

Figure 4.25 (d) shows the three-track invariant mass distribution obtained by assigning the pion masses to the like sign tracks and the kaon mass to the opposite sign track. The hashed histogram shows the wrong sign combination with no signal 


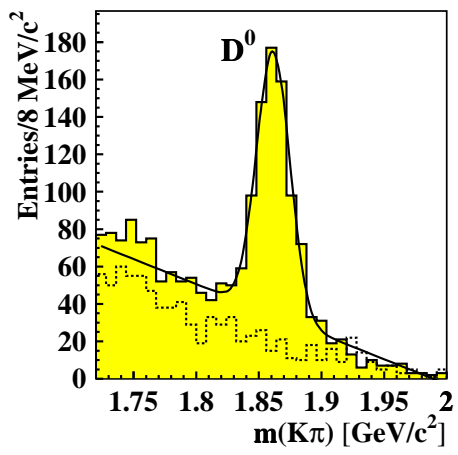

(a)

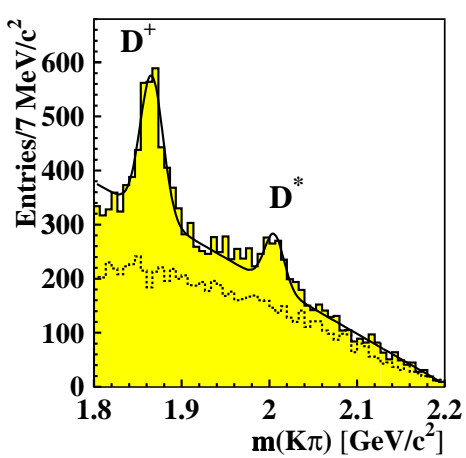

(d)

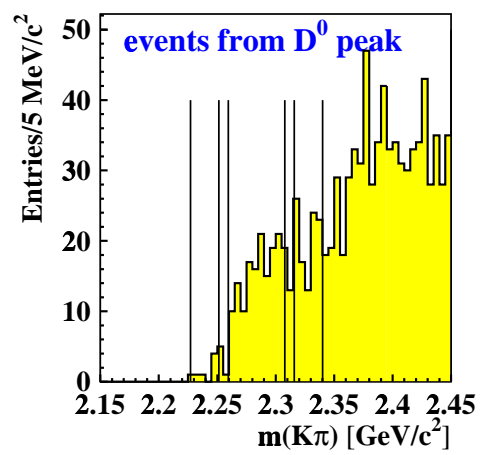

(b)

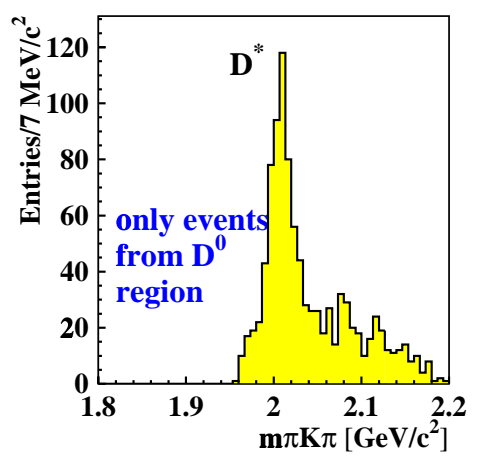

(e)

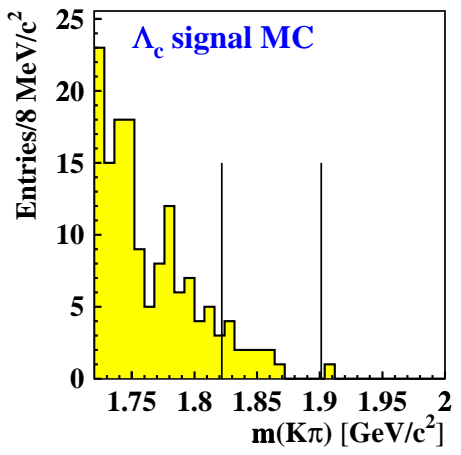

(c)

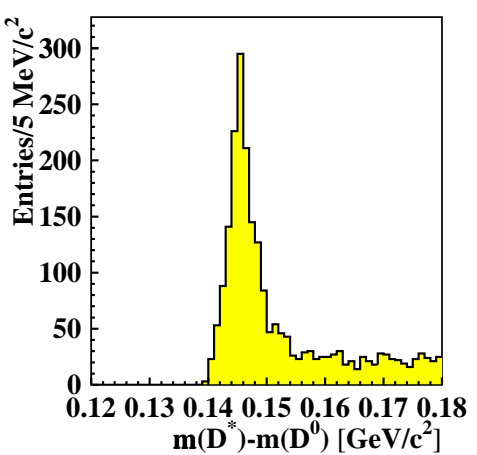

(f)

Figure 4.25: Figure (a) shows the $K \pi$ invariant mass for data, obtained by assigning the pion mass to the proton track. The hashed histograms shows the wrong sign combination, the solid histograms shows the right sign combination. Figure (b) shows the $p K \pi$ invariant mass for data, using only events from the $D^{0}$ peak region. Figure (c) shows the $K \pi$ invariant mass for $\Lambda_{c}$ signal MC. Figure (d) shows the $\pi K \pi$ invariant mass distribution, figure (e) shows the same, only for events from the $D^{0}$ peak region and figure (f) shows $\mathrm{m}(\pi K \pi)$ $\mathrm{m}(K \pi)$ without the application of the $D^{0}$ mass cut.

present. There are two peaks visible in this invariant mass plot. The $D^{+}$peak is described in the next subsection. The $D^{*}$ peak is visible, but not as pronounced as the $D^{0}$ peak found in the $K \pi$ invariant mass plot. It can be enhanced by solely selecting those events whose $K \pi$ invariant mass lies in the $D^{0}$ mass region. This cut also removes the $D^{+}$peak since the $D^{0}$ is not among the decay products of the $D^{+}$. This is shown in figure 4.25 (e). Figure 4.25 (f) shows as a cross check a plot of the difference between the invariant mass of the $D^{*}$ and the invariant mass of the $D^{0}$ without the application of the $D^{0}$ mass cut. The peak lies as expected 


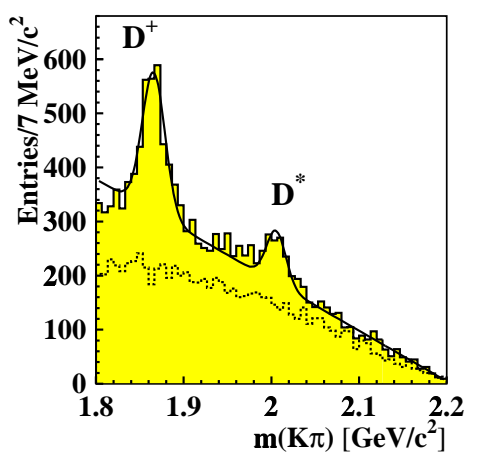

(a)

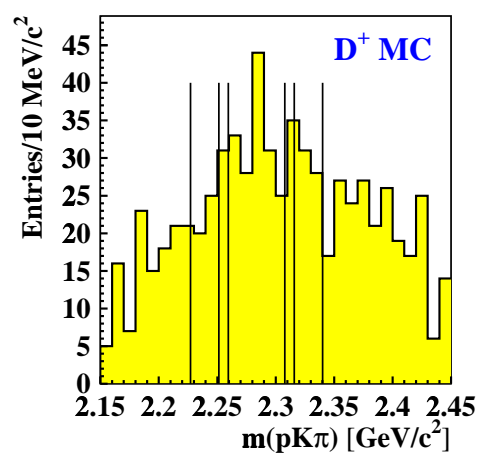

(b)

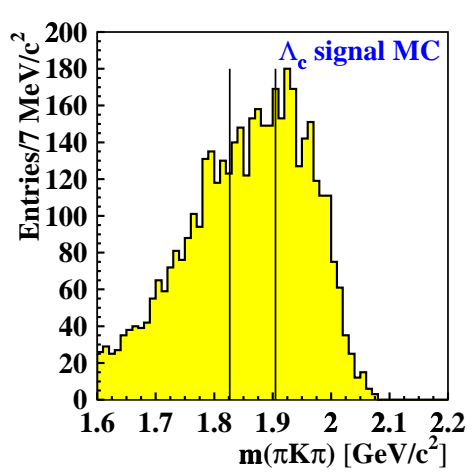

(c)

Figure 4.26: Figure (a) shows the $\pi K \pi$ invariant mass plot from figure 4.25 (d). Figure (b) shows the $p K \pi$ invariant mass distribution obtained for $D^{+}$ MC events. Figure (c) shows the $\pi K \pi$ invariant mass for $\Lambda_{c}$ signal events.

around $145.4 \mathrm{MeV} / \mathrm{c}^{2}$ [1]. To reject background events from $D^{0}$ and $D^{*}$ the two track invariant mass $\mathrm{m}(K \pi)$, obtained by assigning the pion mass to the proton candidate track while maintaining a kaon hypothesis for the kaon candidate track, is required to lie outside $1.8218-1.9012 \mathrm{GeV} / \mathrm{c}^{2}$. This cut removes $D^{0}$ mesons and $D^{*}$ mesons that decay into $D^{0}$ mesons from the candidate sample while keeping almost all $\Lambda_{c}$ events.

\section{Backgrounds from $D^{ \pm}$}

The $D^{+}$mesons visible in the $\pi K \pi$ invariant mass distribution from figure 4.25 (d) can have the following origins:

$$
\begin{aligned}
\bar{B}^{0} \rightarrow & D^{+} l^{-} \bar{\nu}_{l} \\
& \longleftrightarrow \pi^{+} K^{-} \pi^{+}
\end{aligned}
$$

and

$$
\begin{aligned}
& \bar{B}^{0} \rightarrow D^{*}(2010)^{+} l^{-} \bar{\nu}_{l} \\
& \stackrel{D^{+} \pi^{0}}{\longrightarrow} \pi^{+} K^{-} \pi^{+}
\end{aligned}
$$

The proton candidate can thus be a pion from a $D^{+}$decay, which again appears 
in right sign combination with the lepton. Figure 4.26 (a) shows again the $\pi K \pi$ invariant mass plot from figure 4.25 (d).

The $D^{+}$peak lies on top of a significant amount of background. $D^{+} \mathrm{MC}$ was generated to investigate which region in $p K \pi$ invariant mass the true $D^{+}$events populate. This is shown in figure 4.26 (b). The $D^{+}$events populate the $\Lambda_{c}$ signal as well as the sideband region. The MC suggests that there are slightly more events in the signal region compared to the background region. If a cut around the $D^{+}$ mass does not impact the signal events it would still be beneficial. Figure 4.26 (c) shows the $\pi K \pi$ invariant mass for $\Lambda_{c}$ signal events. A large quantity of signal events populate the $D^{+}$mass region and so a cut on this region is not applied here. The slight enhancement of $D^{+}$events in the signal region is accounted for when assigning a systematic uncertainty to the lifetime measurement (chapter 6).

The decay of $D^{+}$mesons can also result in a $K K \pi$ final state, which is Cabibbo suppressed. This decay is treated in the following section together with the background from $D_{s}^{ \pm}$mesons.

\section{Background from $D_{s}^{ \pm}$}

$D_{s}$ mesons occur in decays of the $B_{s}^{0}$ meson: $\bar{B}_{s}{ }^{0} \rightarrow D_{s}^{+} l^{-} \bar{\nu}_{l}$. The $D_{s}^{+}$decays into $\Phi \pi^{+}\left(3.6 \%\right.$ [1]), with the $\Phi$ subsequently decaying to $K^{-} K^{+}$(49\% [1]):

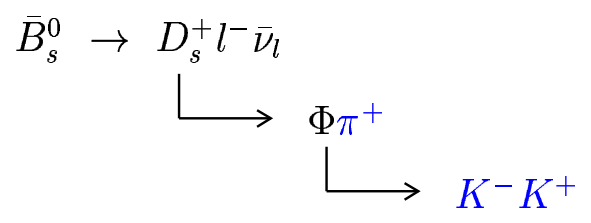

If the three candidate tracks originate from a $D_{s}^{+}$instead of a $\Lambda_{c}^{+}$there are two possibilities for the tracks to be misassigned:

- the proton candidate is really a kaon from the $\Phi$ decay

- the proton is a pion from the $D_{s}$ decay and the pion is a kaon from the $\Phi$ decay 

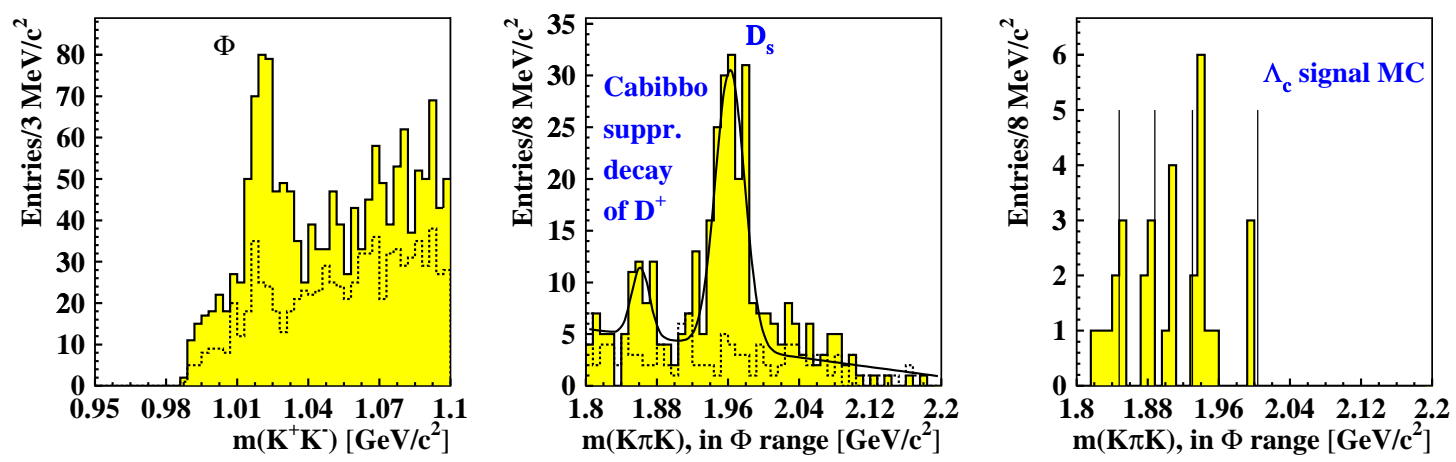

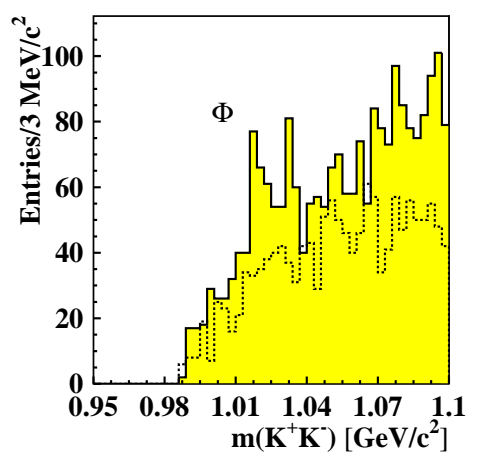

(a)

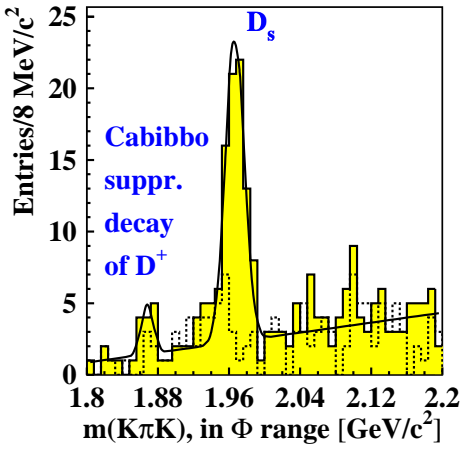

(b)

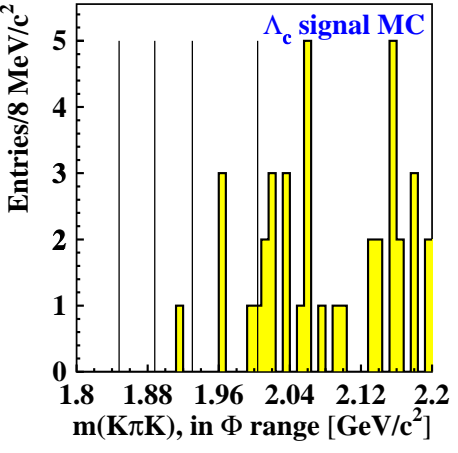

(c)

Figure 4.27: The top row of plots considers the background from $D_{s}$ mesons where the proton candidate is a kaon from a $\Phi$ decay. The bottom row of plots considers the case where the proton candidate is a pion from the $D_{s}$ decay and the pion is a kaon from the $\Phi$ decay. Figure (a) shows the $K^{+} K^{-}$invariant mass for data. Figure (b) shows the $K \pi K$ invariant mass for data events that have a $K^{+} K^{-}$invariant mass around the $\Phi$ mass and figure (c) shows the same plot for $\Lambda_{c}$ signal MC.

Again the lepton-meson combination has the right sign. Figure 4.27 shows the various invariant mass combinations for the first (top row) and second (bottom row) case. Figure 4.27 (a) shows the $K^{+} K^{-}$invariant mass with the $\Phi$ peak visible. The wrong-sign combination also contains a few $\Phi$ meson candidates. Figure 4.27 (b) shows the $K \pi K$ invariant mass for $K K$ combinations in the range of the $\Phi$ mass. Additionally to a clean peak from $D_{s}$ mesons there is a second, smaller peak visible, which originates from Cabibbo suppressed decays of the $D^{+}$. Figure 4.27 (c) shows the same plot for $\Lambda_{c}$ signal MC with lines indicating the $D^{+}$and $D_{s}$ peaks. This figure indicates that cutting out events in those regions does not impact the $\Lambda_{c}$ signal events, which populate lower (higher) invariant masses in the top (bottom) plot of figure 4.27 (c). A cut for both cases of possible misassignments affects less 


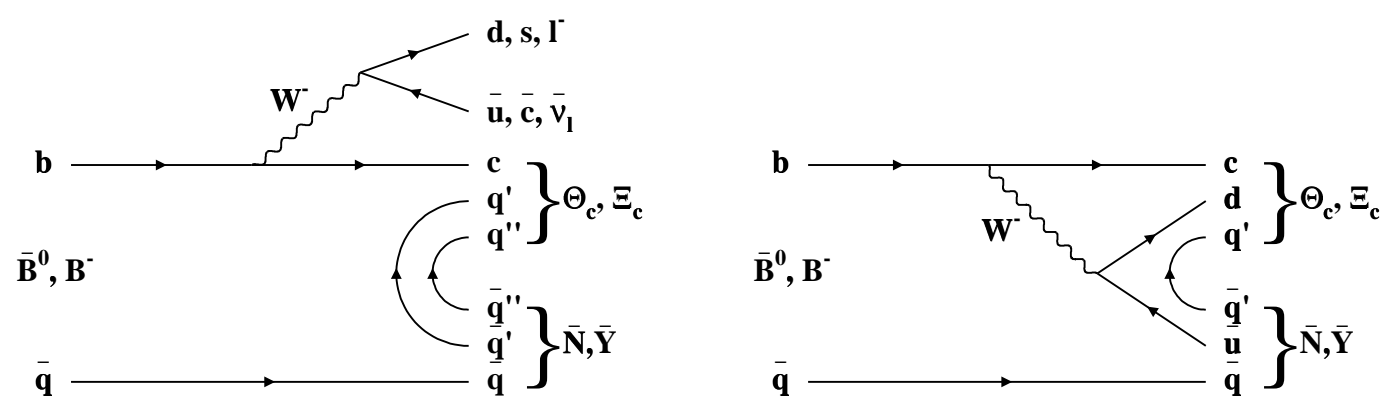

Figure 4.28: Graphs for external (left) and internal (right) W-emission for the decay $B \rightarrow \Lambda_{c} X$. $\Theta_{\mathrm{c}}$ denotes non-strange charmed baryons, and $\mathrm{N}$ and $\mathrm{Y}$ denote nucleons and hyperons respectively.

than one percent of the signal events and is thus applied.

In summary the following anti-selection cuts are applied to reject background from real charm decays:

- $1.8218 \mathrm{GeV} / \mathrm{c}^{2} \leq \mathrm{m}(K \pi) \leq 1.9012 \mathrm{GeV} / \mathrm{c}^{2}$

- $1.01 \mathrm{GeV} / \mathrm{c}^{2} \leq \mathrm{m}\left(K^{+} K^{-}\right) \leq 1.03 \mathrm{GeV} / \mathrm{c}^{2}$ and $1.848 \mathrm{GeV} / \mathrm{c}^{2} \leq \mathrm{m}(K \pi K) \leq$ $1.888 \mathrm{GeV} / \mathrm{c}^{2}$

- $1.01 \mathrm{GeV} / \mathrm{c}^{2} \leq \mathrm{m}\left(K^{+} K^{-}\right) \leq 1.03 \mathrm{GeV} / \mathrm{c}^{2}$ and $1.930 \mathrm{GeV} / \mathrm{c}^{2} \leq \mathrm{m}(K \pi K) \leq$ $2.004 \mathrm{GeV} / \mathrm{c}^{2}$

The latter two cuts are applied for both possible misassignments as described above.

\subsubsection{Other Sources of $\Lambda_{c}$ Baryons}

Additionally to the signal decay, $\Lambda_{b} \rightarrow \Lambda_{c} l \nu_{l}$, there are various other $B$ and $\Lambda_{b}$ decays that can result in the production of $\Lambda_{c}$ baryons, such as:

- $\overline{\mathrm{B}}_{\mathrm{u}, \mathrm{d}} \rightarrow \Lambda_{\mathrm{c}}^{+} \overline{\mathrm{N}} \mathrm{l}^{-} \bar{\nu}_{\mathrm{l}} \mathrm{X}$

- $\overline{\mathrm{B}}_{\mathrm{u}, \mathrm{d}, \mathrm{s}} \rightarrow \Lambda_{\mathrm{c}}^{+} \mathrm{D}_{\mathrm{s}}^{-} \overline{\mathrm{N} X}$

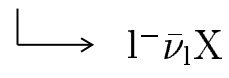

- $\Lambda_{\mathrm{b}}^{0} \rightarrow \Lambda_{\mathrm{c}}^{+} \mathrm{D}_{\mathrm{s}}^{-} \mathrm{X}$

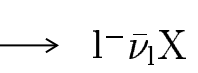


The effect of all of the above processes was however found to be negligible in a similar analysis on the observation of bottom baryon semileptonic decay [52]. Figure 4.28 shows the Feynman diagrams for $B \rightarrow \Lambda_{c} X$. The diagram on the left shows the graph for external W-emission which is responsible for the background processes from $\mathrm{B}$ decays described above. The diagram on the right shows internal $\mathrm{W}$-emission and contributes only to fully hadronic decays.

The appearance of candidates through the first decay is suppressed owing to its low branching fraction and to baryon number conservation. The CLEO Collaboration has set an upper limit of $5.7 \%$ at a $90 \%$ confidence limit [53] for the following ratio of branching fractions:

$$
\frac{\mathcal{B}\left(B \rightarrow \Lambda_{c} \bar{N} l \nu X\right)}{\mathcal{B}\left(B \rightarrow \Lambda_{c} X\right)}
$$

With $\mathcal{B}\left(B \rightarrow \Lambda_{c} X\right)=(6.4 \pm 1.1) \%$ [1] this results in a branching fraction limit of $0.36 \%$ for the process $B \rightarrow \Lambda_{c} \bar{N} l \nu X$. The CLEO collaboration conclude from their study that the simple external W-emission does not saturate the entire decay width of the process $\mathrm{b} \rightarrow$ baryons and that a substantial fraction occurs through internal W-emission.

The above process is further suppressed in the present analysis owing to baryon number conservation, which requires another baryon to be produced together with the $\Lambda_{c}$ baryon. This results in a softer spectrum of the invariant mass of the $1-\Lambda_{c}$ combination, which is shown in the figure to the right. The solid histogram shows the distribution for the realistic MC simulation for the decay $B \rightarrow \Lambda_{c} \bar{p} \mu \bar{\nu}$ and the dashed histogram represents the signal decay (again for realistic MC simulation). The majority of the remaining back-

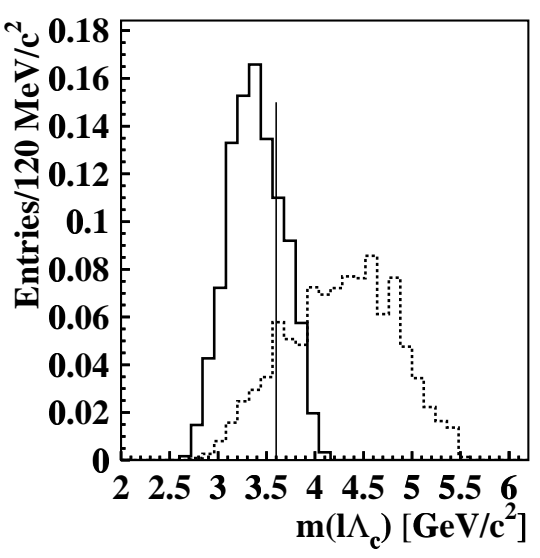

Figure 4.29: Shown is $\mathrm{m}\left(1 \Lambda_{c}\right)$ for the decay $B \rightarrow \Lambda_{c} \bar{p} l \nu$ (solid histogram) and for the signal decay (dashed histogram). ground is rejected by enhancing the cut on $\mathrm{m}\left(1 \Lambda_{c}\right)$ from the kinematic limit, $\mathrm{m}\left(\Lambda_{c}\right)$, to $3.6 \mathrm{GeV} / \mathrm{c}^{2}$, indicated by the vertical line in the above figure.

The second of the above mechanisms for $\Lambda_{c}$ production is again suppressed due to baryon number conservation and also due to the creation of a lepton from a charm 
decay, which has a low branching ratio $\left(\mathcal{B}\left(D_{s} \rightarrow e^{+}+\right.\right.$anything $\left.)=8_{-5}^{+6}[1]\right)$. Furthermore the leptons from charm decays have a softer momentum spectrum [54].

The equivalent decay of the $\Lambda_{b}$ (third decay of the above background processes) is also suppressed due to low the branching fraction and low lepton energy. A similar study concerning the observation of bottom baryons in semileptonic decays concluded that this channel will contribute less than one event [52].

\subsubsection{Background from $b \bar{b}$ and $c \bar{c}$}

If a $b \bar{b}$ or $c \bar{c}$ pair is created via gluon splitting in the final state, the heavy quarks are generated relatively close to each other in direction (in the case of $g g \rightarrow q \bar{q}$ they are created back-to-back). Thus it is possible that the quark decay contributes the lepton and the antiquark contributes the $\Lambda_{c}$ baryon to a candidate, or vice versa. This background will be suppressed by the invariant mass cut $3.6 \leq \mathrm{m}\left(l \Lambda_{c}\right) \leq 5.5$.

In the case of $b \bar{b}$ creation this process yields a wrong-sign combination between the lepton and the $\Lambda_{c}$. A right-sign combination is achieved if the lepton originates instead from the decay of a daughter c quark, which is again further suppressed by the low branching fraction and the low lepton energy.

In the case of $c \bar{c}$ creation through gluon splitting, it is possible to get a right-sign combination through the decays $c \rightarrow \Lambda_{c}^{+} X$ and $\bar{c} \rightarrow l^{-} \bar{\nu}_{l} \bar{d}$. The lepton originates here again from a c-quark resulting in a softer momentum spectrum; furthermore the invariant mass of the lepton- $\Lambda_{c}$ combination is not correlated with $m_{b}$, so that this background is suppressed by the momentum cuts as well as by the cut on the invariant mass of the lepton- $\Lambda_{c}$ combination. Owing to the short mean decay length of the $\Lambda_{c}$ baryon, $c \tau=59.9 \mu \mathrm{m}$ [1], this decay is strongly suppressed by the trigger cut on the impact parameter. The impact of such a prompt background on the lifetime measurement is thus considered negligible $[52,55,56]$.

Further backgrounds, for instance due to fake leptons, have no sign correlation, so that they can be monitored via the wrong-sign combination which shows no signal around the mass of the $\Lambda_{c}$ baryon. Again the combination of a real $\Lambda_{c}$ with a fake lepton is strongly suppressed by the short mean decay length of the $\Lambda_{c}[52,55,56]$. 


\subsection{Summary}

All quality and selection cuts described in section 4.4 .3 are summarised below:

Quality cuts

- vertex fit probability $\geq 0.001$

- $\mathrm{dL}_{\mathrm{xy}}\left(\Lambda_{\mathrm{b}}\right) \leq 0.03 \mathrm{~cm}, \mathrm{dL}_{\mathrm{xy}}\left(\Lambda_{\mathrm{c}}\right) \leq 0.04 \mathrm{~cm}$

Selection cuts

- $\Delta \mathrm{R}_{\max }(\mathrm{l}-\mathrm{pK} \pi) \geq 1.2$

- $\mathrm{p}_{\mathrm{T}} \mathrm{p} \geq 2 \mathrm{GeV} / \mathrm{c}$

- $\mathrm{p}_{\mathrm{T}} \mathrm{K} \geq 0.9 \mathrm{GeV} / \mathrm{c}$

- $\mathrm{p}_{\mathrm{T}} \Lambda_{\mathrm{c}} \geq 5 \mathrm{GeV} / \mathrm{c}$

- $\mathrm{p}_{\mathrm{T}} \mathrm{l} \Lambda_{\mathrm{c}} \geq 9 \mathrm{GeV} / \mathrm{c}$

- $3.6 \mathrm{GeV} / \mathrm{c}^{2} \leq \mathrm{m}\left(\mathrm{l} \Lambda_{\mathrm{c}}\right) \leq 5.5 \mathrm{GeV} / \mathrm{c}^{2}$

- $\mathrm{p}_{\mathrm{T}} \mathrm{p} / \mathrm{p}_{\mathrm{T}} \pi \geq 1.3$

- $\mathrm{L}_{\mathrm{xy}}\left(\right.$ p.v. $\left.-\Lambda_{\mathrm{c}}\right) \geq 0.02 \mathrm{~cm}$

- $-0.015 \mathrm{~cm} \leq \mathrm{c} \tau\left(\Lambda_{\mathrm{c}}\right) \leq 0.035 \mathrm{~cm}$

- ptratio1 $\geq 0.5$

- ptratio $2 \geq 0.25$

- $\operatorname{dedxp} \leq 2.5$

- $\operatorname{dedxk} \leq 2$

- $\operatorname{dedxpi} \leq 1$

Anti-selection cuts to reject background from other charm particles

- $1.8218 \mathrm{GeV} / \mathrm{c}^{2} \leq \mathrm{m}(K \pi) \leq 1.9012 \mathrm{GeV} / \mathrm{c}^{2}$ 


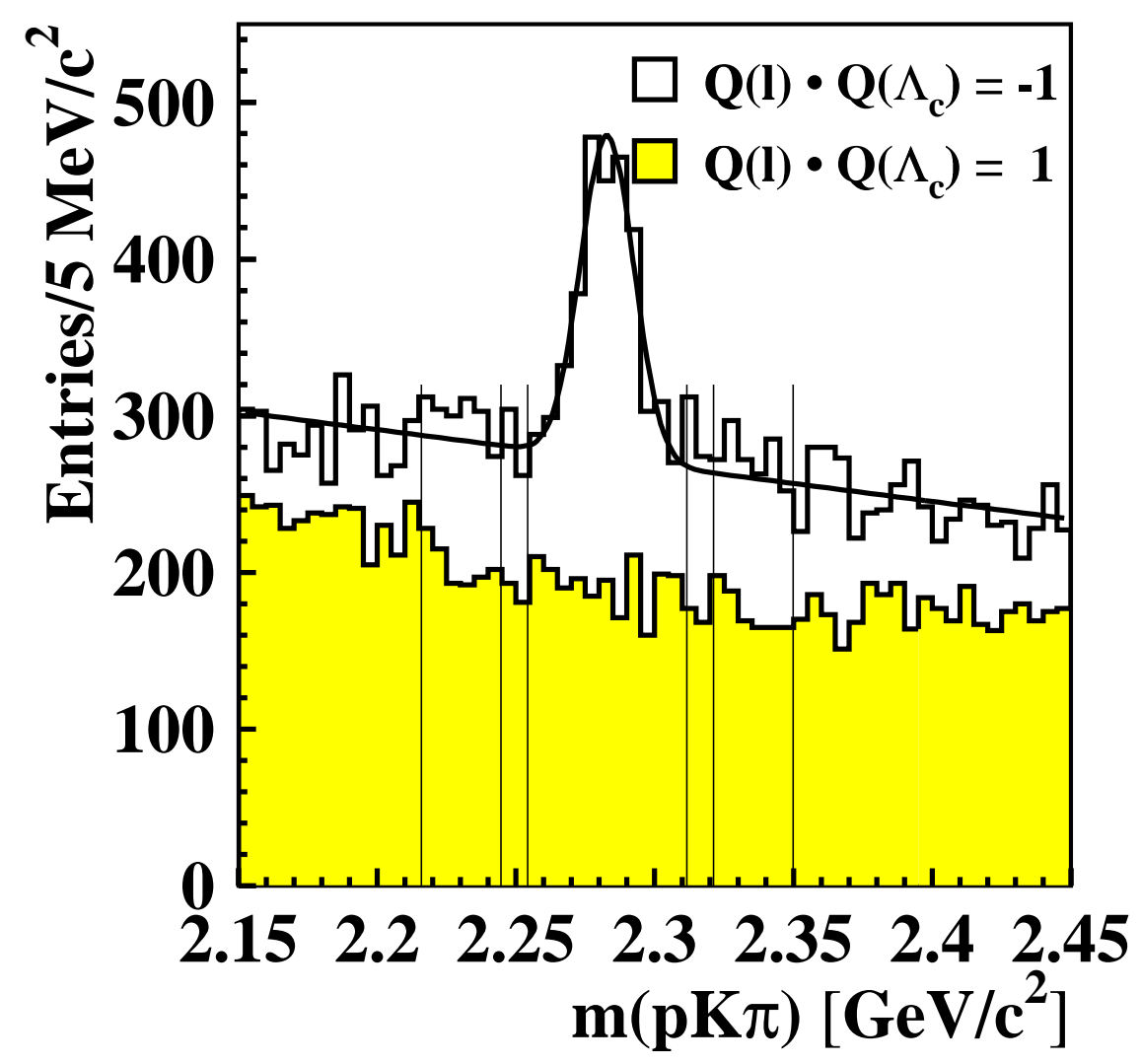

Figure 4.30: $p K \pi$ invariant mass distribution after application of all cuts.

- $1.01 \mathrm{GeV} / \mathrm{c}^{2} \leq \mathrm{m}\left(K^{+} K^{-}\right) \leq 1.03 \mathrm{GeV} / \mathrm{c}^{2}$ and

$1.848 \mathrm{GeV} / \mathrm{c}^{2} \leq \mathrm{m}(K \pi K) \leq 1.888 \mathrm{GeV} / \mathrm{c}^{2}$

- $1.01 \mathrm{GeV} / \mathrm{c}^{2} \leq \mathrm{m}\left(K^{+} K^{-}\right) \leq 1.03 \mathrm{GeV} / \mathrm{c}^{2}$ and

$1.930 \mathrm{GeV} / \mathrm{c}^{2} \leq \mathrm{m}(K \pi K) \leq 2.004 \mathrm{GeV} / \mathrm{c}^{2}$

Figure 4.30 shows the final $p K \pi$ invariant mass distribution, for the right-sign and the wrong-sign events, obtained using all good runs recorded with the mu-SVT trigger after the application of the cuts listed above.

In the following chapter the methodology of the lifetime measurement is discussed, succeeded by a study of the related systematic uncertainties. Chapter 7 then provides the result of the lifetime measurement and conclusions. 


\section{Chapter 5}

\section{Measurement of the $\Lambda_{b}$ Lifetime}

The decays of unstable particles are intrinsically random, i.e. the lifetime of a specific particle is not predictable. The mean lifetime of a particle species however is a physically definite quantity and the aim of this thesis is to determine the mean lifetime of the $\Lambda_{b}$ baryon.

\subsection{Determination of the Proper Decay Length}

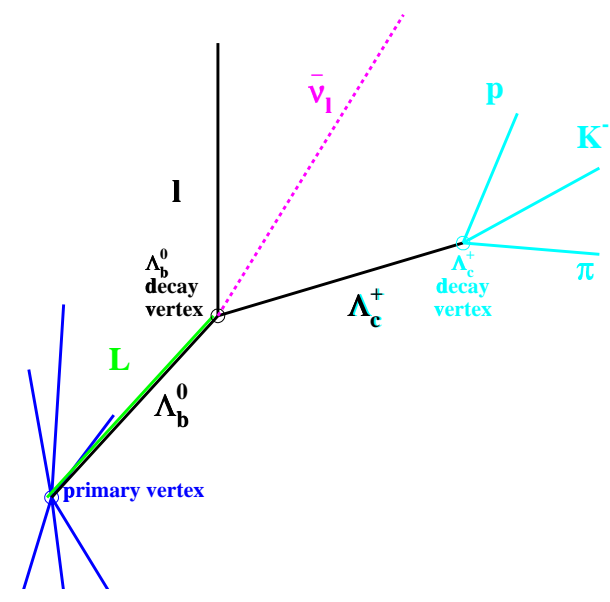

The time $\mathrm{t}$ between the creation of a $\Lambda_{b}$ baryon and its decay is of the order of picoseconds and not accessible to direct measurement. The quantity accessible to experiment is the decay length $L$ depicted in figure 5.1. The $\Lambda_{b}$ particles observed by $\mathrm{CDF}$ travel at high velocities. The proper decay length $X$ is obtained by taking the Lorentz boost of the particle into ac-

Figure 5.1: The figure shows the event topology for the signal $\Lambda_{b}$ decay and the decay length $L$ of the $\Lambda_{b}$. (The decay length of the $\Lambda_{c}$ is greatly exaggerated.)

count: $c \tau \equiv X=L\left(\Lambda_{b}\right) / \beta \gamma ; \tau$ is the lifetime in the particle's restframe.

To determine the mean lifetime, $X$ is reconstructed for every $\Lambda_{b}$ candidate event as:

$$
X=\frac{L_{3 D}\left(\Lambda_{b}\right)}{\beta \gamma}=\frac{L_{3 D}\left(\Lambda_{b}\right) \cdot M\left(\Lambda_{b}\right)}{p\left(\Lambda_{b}\right)}=\frac{L_{x y}\left(\Lambda_{b}\right) \cdot M\left(\Lambda_{b}\right)}{p_{T}\left(\Lambda_{b}\right)} .
$$


The transverse momentum $\mathrm{p}_{\mathrm{T}}\left(\Lambda_{\mathrm{b}}\right)$ is measured by the tracking system; the value of the mass of the $\Lambda_{b}$ baryon, $5.624 \pm 9 \mathrm{GeV} / \mathrm{c}^{2}$, is taken from the PDG [1], and $L_{x y}$, the decay length $L$ projected into the $x y$-plane, is extracted from the vertex fit.

\subsubsection{The K-Factor}

When investigating semileptonic decays, the transverse momentum of the $\Lambda_{b}$ is not directly accessible due to the momentum carried by the undetectable neutrino. Only the transverse momentum of the lepton- $\Lambda_{c}$ combination, and thus the so-called pseudo-proper decay length $X^{\prime}$, is measurable:

$$
c \tau^{\prime} \equiv X^{\prime}=\frac{L_{x y}\left(\Lambda_{b}\right) \cdot M\left(\Lambda_{b}\right)}{p_{T}\left(l \Lambda_{c}\right)} .
$$

By introducing a correction factor $\mathrm{K}$

$$
\mathrm{K}=\frac{p_{T}\left(l \Lambda_{c}\right)}{p_{T}\left(\Lambda_{b}\right)},
$$

the proper decay length can be related to the pseudo-proper decay length via

$$
X=\mathrm{K} \cdot X^{\prime} .
$$

The K-factor varies with the kinematics of the decay due to the variable amount of transverse momentum carried away by the undetected neutrino for any given $\mathrm{p}_{\mathrm{T}}\left(\Lambda_{\mathrm{b}}\right)$. This means that there is not a specific K-factor associated to a certain value of $\mathrm{p}_{\mathrm{T}}\left(1 \Lambda_{\mathrm{c}}\right)$ but rather a distribution of the $\mathrm{K}$-factor values which must be convoluted into the overall correction. This convolution is shown in more detail in section 5.2.1. The K-factor distribution, which will be input into the lifetime fit, has to be extracted from $\mathrm{MC}$ simulations. Owing to correlations between the transverse momenta $\mathrm{p}_{\mathrm{T}}\left(\Lambda_{\mathrm{b}}\right)$ and $\mathrm{p}_{\mathrm{T}}\left(1 \Lambda_{\mathrm{c}}\right)$ and some of the variables used for the candidate selection, the K-factor must be determined after the application of the selection cuts. A comparison of data and MC distributions of the cut variables is shown in appendix A. Within the statistical error of the data distributions the agreement is reasonable. A slight discrepancy can however be noted in the distribution of the transverse momentum of the pion. However no cut is applied on this variable and the cut values used for the other momentum variables are varied to estimate any 


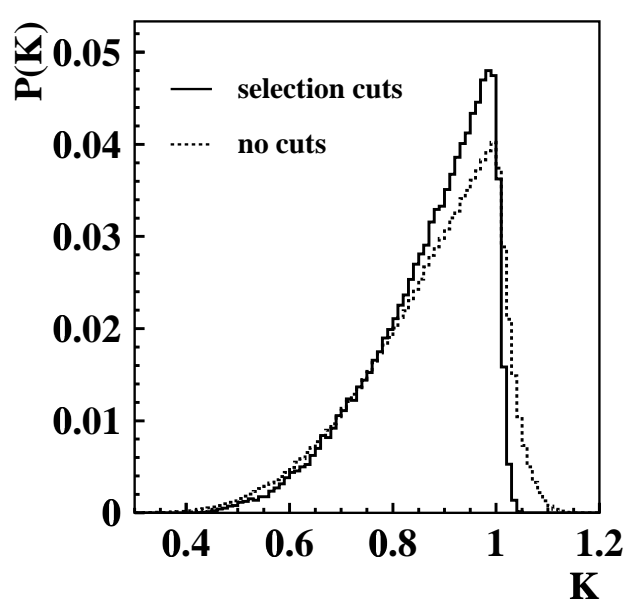

(a)

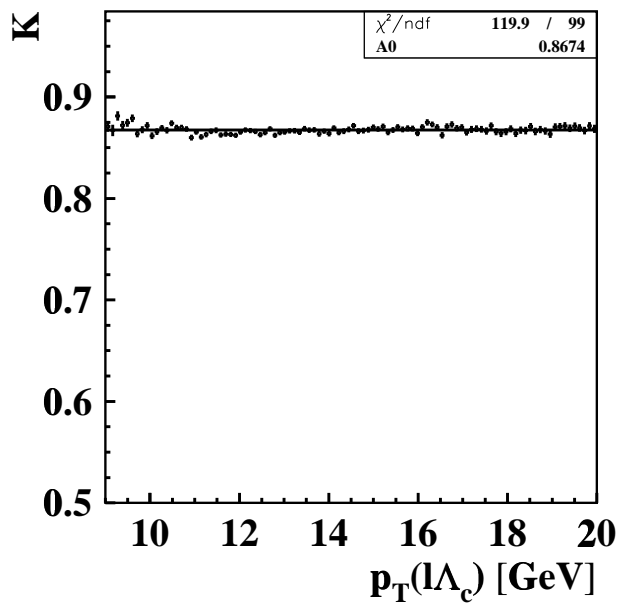

(b)

Figure 5.2: Figure (a) shows the K-factor distribution before and after the application of selection cuts. Figure (b) shows the mean value of the K-factor in bins of $\mathrm{p}_{\mathrm{T}}\left(1 \Lambda_{\mathrm{c}}\right)$.

systematic uncertainty on the lifetime fit that can arise due to incorrect modelling of these distributions (see chapter 6).

Figure 5.2 (a) shows the normalised K-factor distribution determined from the high statistics parametric simulation, generated to describe the data. The dashed and the solid histogram show the K-factor distribution before and after the application of all selection cuts. The dependency on the selection cuts is clearly visible. The mean value of the K-factor is shown in bins of $\mathrm{p}_{\mathrm{T}}\left(1 \Lambda_{\mathrm{c}}\right)$ in figure $5.2(\mathrm{~b})$ after the application of all selection cuts. It can be seen that this mean value is constant at a value of 0.87 over the range of $\mathrm{p}_{\mathrm{T}}\left(1 \Lambda_{\mathrm{c}}\right)$ values relevant for this analysis.

\subsubsection{The Trigger Bias}

An additional concern for the lifetime measurement is the introduction of the trigger cut on the impact parameter. As described in section 4, triggering on the impact parameter enhances the content of events that contain particles originating from a displaced vertex, i.e. events with lifetime. This introduces a bias to the lifetime measurement. The unbiased proper lifetime distribution follows an exponential law, i.e. a large fraction of $\Lambda_{b}$ baryons decay after a short time and their decay products 


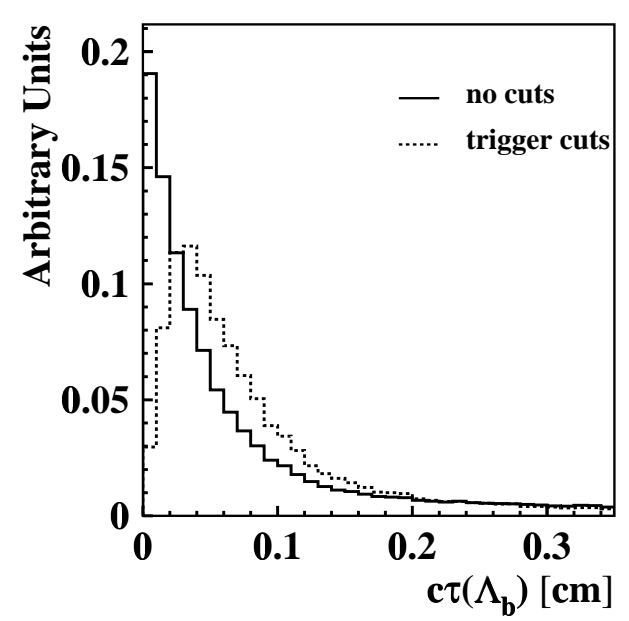

(a)

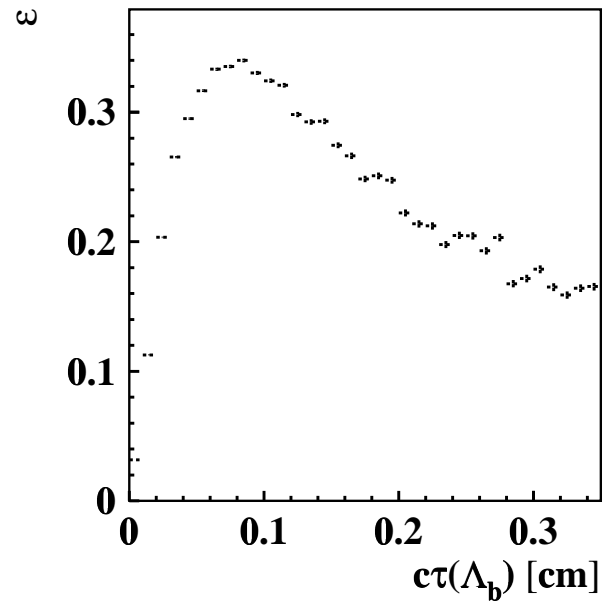

(b)

Figure 5.3: Figure (a) shows the distribution of $c \tau\left(\Lambda_{b}\right)$ before and after application of the trigger cuts. The distributions are normalised. Figure (b) shows the ratio of the two distributions from (a) (dashed histogram divided by solid histogram) before their normalisation.

have a relatively short impact parameter. Through the cut on the impact parameter, longer-lived $\Lambda_{b}$ baryons are selected preferably to shorter-lived ones. This cut thus causes a shift of the $c \tau$ distribution to higher values. Figure 5.3 (a) depicts this phenomenon, showing the normalised $c \tau$ distribution, determined from the parametric simulation, before and after the application of the trigger cuts (solid and dashed histogram). The shift to larger values after the application of the cuts is clearly visible. This bias introduced by the trigger is accounted for in the lifetime fit through the introduction of a $c \tau$ efficiency curve. This curve is obtained from the parametric simulation by dividing the $c \tau$ distribution, obtained after application of the respective cuts including the trigger cuts, by the $c \tau$ distribution, obtained after application of all but the trigger cuts. Figure 5.3 (b) shows this ratio for the distributions from figure 5.3 (a). The ratio is built from the original distributions without normalisation; the normalisation was only used for the distributions in figure 5.3 (a) to highlight the shift in $c \tau$. Before the $c \tau$ efficiency and the K-factor distribution are extracted from the parametric simulation after the application of the respective cuts, the generator level quantities are corrected for various detector effects [51]. For what follows it is important to note that the K-factor and the $c \tau$ efficiency distribution 
are always extracted from the parametric simulation. Before the application of the lifetime fit to the data the fit is tested on the realistic simulation, i.e. the simulation that includes the detector geometry as well as a simulation of the secondary vertex trigger. The detector description in the realistic simulation is not perfect, so that some of the corrections will differ depending on whether the parametric simulation is adjusted to fit the realistic simulation or the data. Additionally some detector effects are run dependent and, as the realistic simulation is composed of only one run, this introduces further differences between data and realistic simulation. Thus the K-factor and the $c \tau$ efficiency distribution must be extracted twice, once for use in the lifetime fit to the realistic simulation and once for use in the fit to data.

The corrections applied are explained in the following.

\section{SVT Tracking Efficiency}

Not every physical track will be identified by the secondary vertex trigger SVT. This impact parameter dependent efficiency for identifying a track is referred to as the SVT tracking efficiency or SVT efficiency. The SVT efficiency is run dependent. Furthermore the efficiency simulated in the realistic simulation is generally lower than the true efficiency observed in data. The realistic simulation used in this analysis is generated according to data run 142110 and figure 5.4 (a) shows the SVT efficiency for this sample of the realistic MC. Figure 5.4 (b) shows the SVT efficiency curve for data which was obtained from an inclusive $J / \psi$ sample [57]. The runs in the $J / \psi$ sample are weighted according to the number of events that pass the lepton+SVT trigger for the respective run. The curve fitted to the SVT efficiency is parametrised by the function

$$
\varepsilon_{\mathrm{SVT}}(\mathrm{d} 0)=0.5 \cdot \mathrm{P} 1 \cdot \operatorname{erfc}((|\mathrm{d} 0|-\mathrm{P} 2) / \mathrm{P} 3), \text { with } \operatorname{erfc}(x)=\frac{2}{\sqrt{\pi}} \int_{x}^{\infty} e^{-t^{2}} d t .
$$

The parameters extracted from $\mathrm{MC}$ run 142110 are $\mathrm{P} 1=0.546 \pm 0.003, \mathrm{P} 2=0.133$ $\pm 0.003 \mathrm{~cm}$ and $\mathrm{P} 3=0.053 \pm 0.006 \mathrm{~cm}$. The parameters for data are $\mathrm{P} 1=0.697$ $\pm 0.001, \mathrm{P} 2=0.177 \pm 0.003 \mathrm{~cm}$ and $\mathrm{P} 3=0.075 \pm 0.004 \mathrm{~cm}[57,58]$.

\section{The Impact Parameter Resolution}

To account for the two correlated impact parameter resolutions of the secondary 


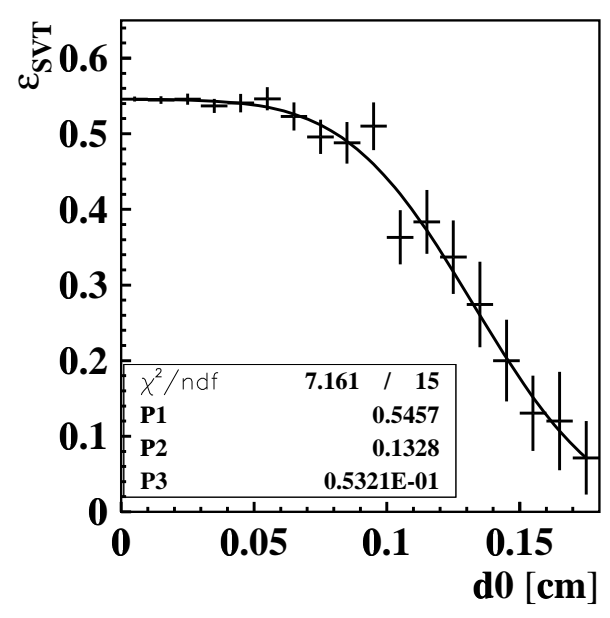

(a)

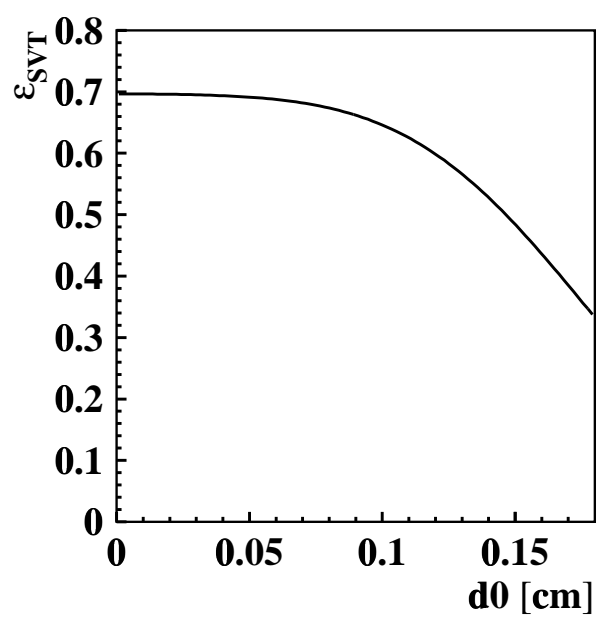

(b)

Figure 5.4: The SVT tracking efficiency as a function of d0 for the realistic simulation of run 142110 (a) and for data (b) obtained from an inclusive $J / \psi$ sample [57].

vertex trigger $\left(\sigma_{S V T}\right)$ and the $\operatorname{SVX}\left(\sigma_{S V X}\right)$, the impact parameter of a simulated track, derived from the generator level quantities, is smeared with a double Gaussian:

$$
f_{G}\left(\rho, \mathrm{d} 0_{\mathrm{SVT}}, \mathrm{d} 0_{\mathrm{SVT}}\right)=\exp \left[\frac{-0.5}{1-\rho^{2}} \cdot\left(\frac{\mathrm{d} 0_{\mathrm{SVT}}^{2}}{\sigma_{\mathrm{SVT}}^{2}}-2 \frac{\rho \mathrm{d} 0_{\mathrm{SVT}} \mathrm{d} 0_{\mathrm{SVX}}}{\sigma_{\mathrm{SVT}} \sigma_{\mathrm{SVX}}}+\frac{\mathrm{d} 0_{\mathrm{SVX}}^{2}}{\sigma_{\mathrm{SVX}}^{2}}\right)\right] ;
$$

$\rho$ is the correlation coefficient. The values used to adjust the parametric simulation to data are: $\rho=0.8, \sigma\left(\mathrm{d}_{\mathrm{SVT}}\right)=57 \mu \mathrm{m}, \sigma\left(\mathrm{d} 0_{\mathrm{SVX}}\right)=45 \mu \mathrm{m}$. The values extracted from the realistic simulation are: $\rho=0.8, \sigma\left(\mathrm{d}_{\mathrm{SVT}_{\mathrm{SV}}}\right)=48 \mu \mathrm{m}, \sigma\left(\mathrm{d} 0_{\mathrm{SVX}}\right)=41 \mu \mathrm{m}$.

\section{The Ladder Bow}

The silicon wafers have a bow mainly due to the wafer processing such as cutting and glueing. Partly the bow is also caused due to their own weight. Gravity accounts for bows of 5-10 microns, but the other effects can cause bows up to 50 microns. The bow of silicon ladders is known and accounted for during the track reconstruction for the data and for the realistic simulation. The bow however is not simulated in the realistic simulation so that the reconstruction code will cause an overcorrection. As a result the reconstructed impact parameter of the realistic simulation is shifted slightly compared to the impact parameter calculated from generator level quantities. The shift is at the 0-10 micron level and as it varies for different values of the 


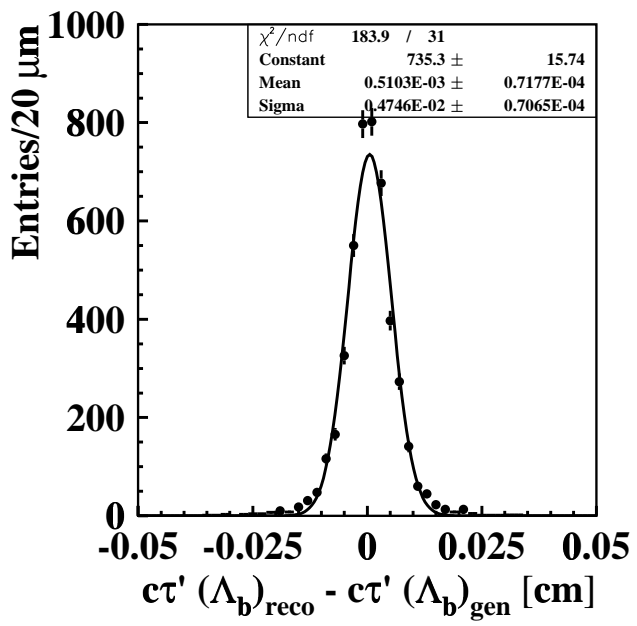

(a)

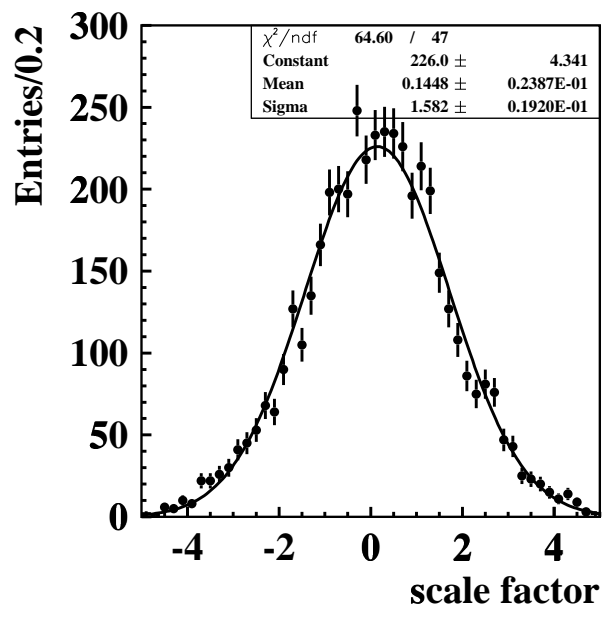

(b)

Figure 5.5: Figure (a) shows the difference between the reconstructed and the generated pseudo proper decay length. Figure (b) shows the scale factor, $\left(c \tau_{\text {reco }}^{\prime}-c \tau_{\text {gen }}^{\prime}\right) / \sigma\left(c \tau_{\text {reco }}^{\prime}\right)$ for run 142110 of the realistic simulation.

impact parameter it is not included as a global shift in the parametric simulation but rather considered a systematic effect for which an error is assigned. The bow effect only concerns the fit to the realistic simulation. However as it is unknown at present whether the overcorrection of the ladder bow in the realistic simulation is responsible for the whole of the radial shift observed, the systematic uncertainty due to this radial shift will also be assigned in the fit to data.

The bow itself is believed to cause the slight shift observed between the reconstructed and the generated $c \tau^{\prime}$ values in the realistic simulation. Figure 5.5 (a) shows the histogram of $c \tau_{\text {reco }}^{\prime}-c \tau_{\text {gen }}^{\prime}$. The visible shift of approximately $5 \mu \mathrm{m}$ is accounted for in the fit to the realistic simulation.

It is convenient to introduce here the error scale factor $s$, a further parameter used as input to the lifetime fit. It is defined as

$$
s=\frac{c \tau_{\text {vertexfit }}^{\prime}-c \tau_{\text {generated }}^{\prime}}{\sigma\left(c \tau_{\text {vertexfit }}\right)},
$$

and the width of this distribution indicates whether the errors from the vertex fit are over- or underestimated. If the errors are correctly assigned this distribution has a width of one. Figure 5.5 (b) shows the error scale factor distribution for run 


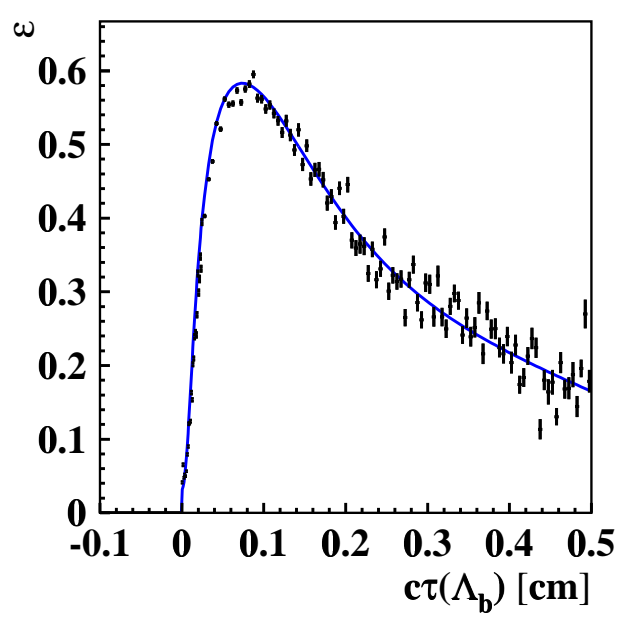

(a)

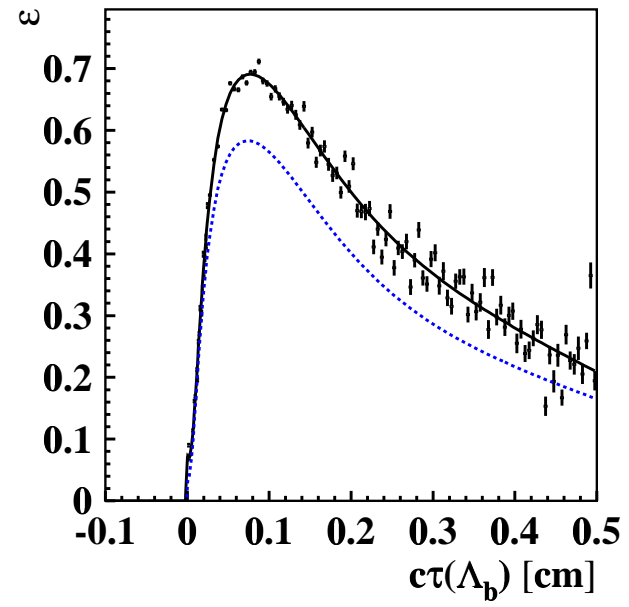

(b)

Figure 5.6: Figure (a) shows the $c \tau$ efficiency curve for run 142110 of the realistic simulation. In figure (b) the same curve is shown (blue/dashed) together with the curve for data (black/solid histogram with fitted line).

142110 of the realistic simulation. The width of 1.6 indicates an underestimation of the errors which will be accounted for in the lifetime fit. The error scale factor for data is determined to be 1.22 from a data sample that was recorded without the application of the impact parameter cut to allow for prompt events [57,59].

After the inclusion of the various corrections explained above, i.e. after application of the SVT tracking efficiency as well as the impact parameter resolution to the tracks of the parametric simulation, the $c \tau$ efficiency curve can be extracted. This has to be done twice since the corrections are dependent on whether the curve is to be extracted for use with the realistic simulation or the data. The values used in the respective case are summarised in table 5.1. Figure 5.6 (a) and (b) show the resulting $c \tau$ efficiency curve for the realistic simulation and the data respectively. The difference in height of the two curves is mainly caused by the different SVT tracking efficiencies applied to the tracks of the parametric simulation. The $c \tau$ efficiency curve is parametrised as:

$$
\varepsilon(x)=A \cdot(x+p) \cdot\left(e^{-\lambda_{1} x}+e^{-\lambda_{2} x}+r\right)+t+u \cdot e^{-\frac{(x-v)^{2}}{2 w^{2}}} .
$$

Table 5.2 shows the values returned by a fit to the histograms of figure 5.6 using the parametrisation of equation 5.2. 


\begin{tabular}{|c|c|c|}
\hline Parameter & for $\mathbf{M C}$ & for data \\
\hline $\mathrm{P} 1$ & 0.546 & 0.697 \\
\hline $\mathrm{P} 2$ & $0.133 \mathrm{~cm}$ & $0.177 \mathrm{~cm}$ \\
\hline $\mathrm{P} 3$ & $0.053 \mathrm{~cm}$ & $0.075 \mathrm{~cm}$ \\
\hline$\sigma\left(\mathrm{d} 0_{\mathrm{SVT}}\right)$ & $48 \mu \mathrm{m}$ & $57 \mu \mathrm{m}$ \\
\hline$\sigma\left(\mathrm{d} 0_{\mathrm{SVX}}\right)$ & $41 \mu \mathrm{m}$ & $45 \mu \mathrm{m}$ \\
\hline$\rho$ & 0.8 & 0.8 \\
\hline
\end{tabular}

Table 5.1: Parameters of the SVT efficiency (P1, P2 and P3) and impact parameter resolution for data and $\mathrm{MC}$ run 142110.

\begin{tabular}{|c|c|c|}
\hline Parameter & for MC & for data \\
\hline$A$ & 14.156 & 14.531 \\
\hline$\lambda_{1}$ & 16.460 & 16.139 \\
\hline$\lambda_{2}$ & 63.900 & 55.916 \\
\hline$p$ & -0.025 & -0.029 \\
\hline$r$ & -0.034 & -0.045 \\
\hline
\end{tabular}

\begin{tabular}{|c|c|c|}
\hline Parameter & for MC & for data \\
\hline$t$ & 0.392 & 0.513 \\
\hline$u$ & 1.034 & 1.231 \\
\hline$v$ & -0.013 & -0.014 \\
\hline$w$ & -0.009 & -0.009 \\
\hline
\end{tabular}

Table 5.2: Parameters obtained from a fit to the histograms in figure 5.6 using equation 5.2 as fit function.

\subsection{The Lifetime Fit}

In this section the fit method is explained, followed by two short examples that apply this method to a fit to exponential decays. This is followed by a description of the adaptation of the fit method to this analysis and tests of the fit that demonstrate the satisfactory behaviour of the fitting routines and method.

\subsubsection{The Unbinned Maximum Log Likelihood Fit}

The fit method employed for the lifetime measurement is an unbinned maximum $\log$ likelihood fit. The starting point of such a fit consists of $n$ measured values $x_{i}$ $(i=1, \ldots, n)$ that are distributed according to a certain probability density function $p(x, \boldsymbol{\alpha})$. The set of parameters $\boldsymbol{\alpha}$ is to be determined in the fit by maximising the joint probability

$$
\mathcal{L}(\boldsymbol{\alpha})=\prod_{i} p\left(x_{i}, \boldsymbol{\alpha}\right)
$$

From a calculational point of view sums are easier to treat than products, so equation 5.3 is transformed through the application of a logarithm on either side, which 
gives the fit method its name, to:

$$
\ln \mathcal{L}(\boldsymbol{\alpha})=\sum_{i} \ln \left(p_{i}\right)
$$

In practise the parameters $\alpha_{i}$ that minimise the negative log likelihood are sought:

$$
-\ln \mathcal{L}(\boldsymbol{\alpha})=-\sum_{i} \ln \left(p_{i}\right)
$$

It is important to note that the probability density function $p$ is normalised. If the minimisation is carried out with the minimisation package MINUIT [60], as is done in this analysis, a factor of two is required, resulting in $-2 \ln \mathcal{L}$. While this factor does not change the location of the minimum it is necessary for the determination of a correct error on the result.

The fit method is "unbinned", since single measurements, instead of bin contents, are used to determine the joint probability.

As an example consider the distribution $p=A e^{-x / c \tau}$, which describes a normal exponential decay, i.e. a decay where no effects such as missing momenta of neutrinos present in the decay or trigger efficiencies are present. Here $x$ refers to the measured (or generated) $c \tau$ values. The constant $A$ is determined from the normalisation condition for $p$ :

$$
\int_{o}^{\infty} A e^{-\frac{x}{c \tau}} d x=1
$$

The resulting probability density function for this case is thus:

$$
p=\frac{1}{c \tau} e^{-\frac{x}{c \tau}}
$$

Here $\boldsymbol{\alpha}$ simply reduces to the parameter $c \tau$ which is determined in the fit by minimising

$$
-2 \sum_{i} \ln \left(\frac{1}{c \tau} e^{-\frac{x_{i}}{c \tau}}\right) .
$$

The sum goes over all measured values of $c \tau$ of a large sample of decaying particles. This minimisation was applied to a set of MC events, generated according to the above probability density function with an input lifetime of $368 \mu \mathrm{m}$. The unbinned $\log$ likelihood fit returned a lifetime of $368.7 \pm 3.7 \mu \mathrm{m}$, which is in good agreement with the input lifetime. The $c \tau$ distribution of the generated events together with the fit result are shown in figure 5.7 (a). 


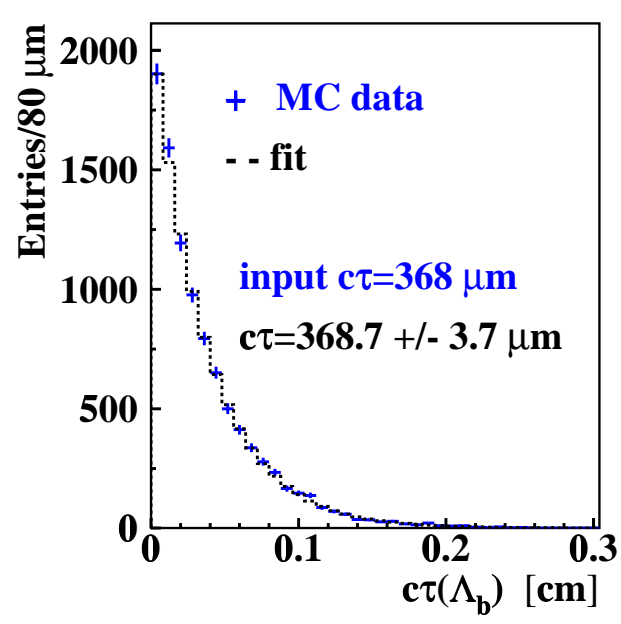

(a)

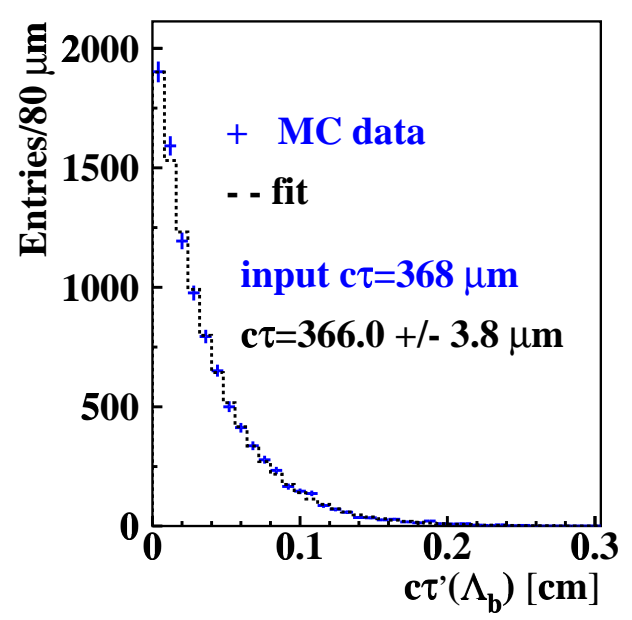

(b)

Figure 5.7: Figure (a) and (b) show the $c \tau$ and $c \tau^{\prime}$ distribution for MC events, generated with an input lifetime of $368 \mu \mathrm{m}$ (points with error bars). The dashed histograms show the result of an unbinned log likelihood fit to these distributions.

A more realistic example is the case where the pseudo proper decay length $X^{\prime}=$ $X / K$ of an exponential decay is measured, as is the case in the signal decay of this thesis. The signal function is then a convolution of the exponential with a normalised $\mathrm{K}$-factor distribution $P(K)$ such as in figure 5.2. If $P(K)$ is available in the form of a histogram, the integration of the convolution turns into a sum and $p$ becomes:

$$
p=\sum_{K} A(K, \tau) e^{-\frac{K X^{\prime}}{c \tau}} P(K) .
$$

$A(K, \tau)$ is again determined from the normalisation condition for $\mathrm{p}$ :

$$
\int_{o}^{\infty} \sum_{K} A(K, \tau) e^{-\frac{K X^{\prime}}{c \tau}} P(K) d X^{\prime}=1 .
$$

As $P(K)$ is itself normalised this yields $A(K, \tau)=K / c \tau$. The probability density function that is input into the Likelihood fit is thus:

$$
p=\sum_{K} \frac{K}{c \tau} e^{-\frac{K X^{\prime}}{c \tau}} P(K) .
$$

Figure 5.7 (b) shows again a fit to a lifetime distribution with a generated $c \tau$ of 368 $\mu \mathrm{m}$. Now the input to the fit are the pseudo $c \tau$ values $X^{\prime}=c \tau^{\prime}$ instead of $X$ itself. The fit returned a lifetime of $366.0 \pm 3.7 \mu \mathrm{m}$, which again agrees well with the input lifetime. 


\subsubsection{The Fit Function}

The fit function that is minimised to obtain the mean lifetime for the $\Lambda_{b}$ baryon is now described. In the two examples given above, a sample of pure signal events was simulated. However in the case of the experimental analysis, the "signal" sample contains a substantial amount of background events as can be seen from the $\mathrm{pK} \pi$ invariant mass distribution (see figure 5.8). Thus the function describing the $c \tau^{\prime}$ distribution of the events in the signal sample must be composed of terms $\mathcal{F}_{s}$ and $\mathcal{F}_{b}$, which describe the signal and background events respectively:

$$
\mathcal{F}=\left(1-f_{b}\right) \mathcal{F}_{s}+f_{b} \mathcal{F}_{b}
$$

The background fraction $f_{b}$ is obtained by a five parameter fit of a Gaussian and a first-order polynomial to the $\Lambda_{c}$ peak in the $p K \pi$ invariant mass plot:

$$
p(x)=p 1 \cdot \exp \left\{-0.5 \cdot[(x-p 2) / p 3]^{2}\right\}+p 4+p 5 \cdot x .
$$

Figure 5.8 shows this $p K \pi$ invariant mass plot for right-sign events after the application of all cuts together with the resulting fitted curve. The Gaussian fit parameters $p 2=\mu_{G}$ and $p 3=\sigma_{G}$ then allow the definition of a signal region $\mu_{G} \pm 3 \sigma$ and sideband regions $\mu_{G} \pm(4-7) \sigma$, which are indicated in figure 5.8 by the yellow (light-grey) and green (dark-grey) bands respectively.

The events in the sideband region are assumed to be pure background events that are described by the background function $\mathcal{F}_{b}$ and are input to the log likelihood fit. The likelihood function $\mathcal{L}$ is then a sum over all events in the signal $\left(N_{s}\right)$ and in the sideband $\left(N_{b}\right)$ region:

$$
\ln \mathcal{L}=\sum_{i=1}^{N_{s}} \ln \mathcal{P}+\sum_{i=1}^{N_{b}} \ln \mathcal{P}_{b}
$$

$\mathcal{P}$ and $\mathcal{P}_{b}$ are the normalised versions of the functions $\mathcal{F}$ and $\mathcal{F}_{b}$. The composition of $\mathcal{F}$ out of $\mathcal{F}_{s}$ and $\mathcal{F}_{b}$ was introduced in equation 5.6. The signal function $\mathcal{F}_{s}$ differs from the example given in section 5.2.1 in two ways. If the decay length originates from a measurement, instead of from generator level quantities, the function for the exponential decay has to be convoluted with a Gaussian to account for the detector 


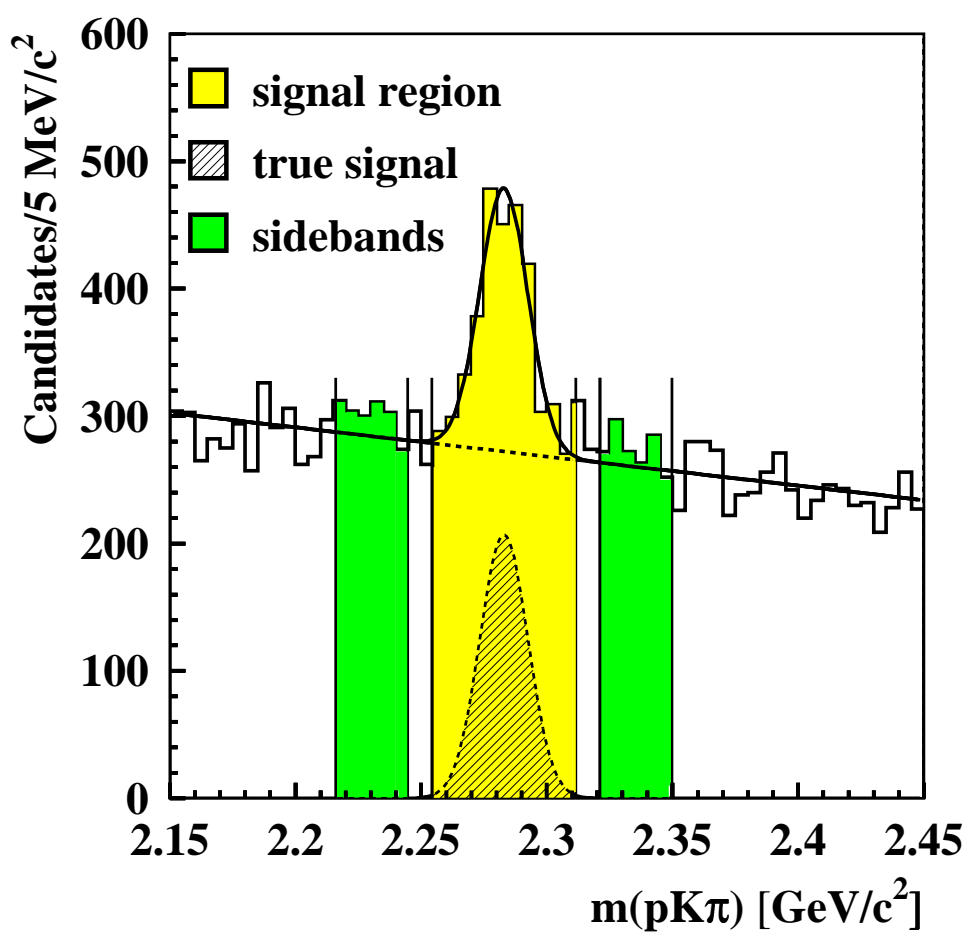

Figure 5.8: Fit of a Gaussian and a first order polynomial to the right-sign $p K \pi$ invariant mass distribution. The signal region and the sidebands are indicated by the yellow (light-grey) and green (dark-grey) bands respectively. The hashed histogram indicates the estimated contribution of true signal events to events in the signal region.

resolution. Furthermore the exponential must be multiplied by the $c \tau$ efficiency $(\varepsilon)$ caused by the trigger cut on the impact parameter:

$$
\mathcal{F}_{s}=\exp \left(K, c \tau^{\prime}\right) \cdot \varepsilon \otimes G \otimes P(K)
$$

The convolution with the K-factor turns into a sum over all bins of $P(K)$ and the function reads:

$$
\mathcal{F}_{s}=\int_{-\infty}^{\infty} d y \sum_{K} P(K) \theta(x-y) e^{\frac{-K(x-y)}{c \tau}} \varepsilon(K(x-y)) \frac{1}{s \sigma \sqrt{2 \pi}} e^{\frac{-y^{2}}{2(s \sigma)^{2}}} .
$$

The $c \tau$ efficiency function $\varepsilon$ is parametrised in equation 5.2. The input values for every event to the fit are $x=c \tau^{\prime}, \sigma=\sigma_{c \tau^{\prime}}$ and the error scale factor $s$. For run 142110 of the realistic simulation the error scale factor is $s=1.6$, for data it was determined to be 1.22 [57]. The step function $\theta(x-y)$ is defined to be one, if its 
argument is larger than zero, i.e. for $y<x$, and as zero, if its argument is less than zero. Appendix B contains some explicit calculations to solve the above integral, which in turn must be normalised for input into the fit.

The background function $\mathcal{F}_{b}(x)$ is parametrised as a $\delta$-function plus an exponential with positive lifetime, indicating that the background is modelled to have a prompt component and a single exponentially decaying component. These are again convoluted with a Gaussian resolution function:

$$
\mathcal{F}_{b}(x)=(\delta(x)+\exp (x)) \otimes G=\delta(x) \otimes G+\exp (x) \otimes G=f_{\delta}+f_{\exp } .
$$

The convolution of a $\delta$-function with a Gaussian yields a Gaussian:

$$
f \otimes \delta=\int f(y) \delta(x-y) d y=f(x)
$$

and the normalised contribution of the $\delta$ term to the fit function is:

$$
f_{\delta}=\frac{1}{\sqrt{2 \pi} \sigma_{0}} \cdot \exp \left[-0.5\left(\frac{x-X_{0}}{\sigma_{0}}\right)^{2}\right] .
$$

The convolution of the exponential with the Gaussian yields after normalisation:

$$
f_{\exp }=\frac{1}{c \tau_{b}} \cdot \exp \left[\frac{1}{2}\left(\frac{\sigma_{b}}{c \tau_{b}}\right)^{2}-\frac{x-X_{b}}{c \tau_{b}}\right] \cdot\left[1-\text { freq }\left(\frac{\sigma_{b}}{c \tau_{b}}-\frac{x-X_{b}}{\sigma_{b}}\right)\right],
$$

with the frequency function

$$
\operatorname{freq}(x)=\frac{1}{\sqrt{2 \pi}} \int_{-\infty}^{x} e^{-\frac{1}{2} t^{2}} d t .
$$

The derivation of the above convolution is shown in Appendix B. The normalised background function $\mathcal{P}_{b}$ is then

$$
\mathcal{P}_{b}=(1-f r a c 1) \cdot f_{\delta}+(f r a c 1) \cdot f_{\exp } .
$$

The fit parameter frac 1 is the ratio of the prompt background to the exponentially decaying background and it is introduced to ensure that the background function is normalised. In the $\log$ likelihood fit $-2 \ln \mathcal{L}$ is then minimised, with $\ln \mathcal{L}$ given in equation 5.7.

This fit has $i=$ seven free parameters $\alpha_{i}$ : $\boldsymbol{\alpha}=\left(c \tau, f r a c 1, \sigma_{0}, X_{0}, c \tau_{b}, X_{b}, \sigma_{b}\right)$ and the minimisation is carried out using the MINUIT package [60]. 


\subsection{Validation of the Fit}

The fitting method, using the signal function necessary for this analysis, was tested on simulations prior to use on data to ensure that no programming errors or fitting biases are present. The test was devised of several steps:

- the fit was used in a blind test to determine the lifetime of several input samples with unknown lifetime

- larger sets of toy experiments with known lifetime were generated to determine the pull distribution for the $c \tau$ value

- upon satisfactory results of the previous tests the fit was tested on the realistic simulation.

\subsubsection{Toy Monte Carlo Studies}

For the blind test four sets of signal toy $\mathrm{MC}$ were generated. The input lifetime for each set was randomly chosen from values in the vicinity of the mean $\Lambda_{b}$ lifetime [61]. Each of those MC samples consists of pairs of $c \tau^{\prime}$ and $\sigma_{c \tau^{\prime}}$ values. The $c \tau^{\prime}$ values were randomly drawn from the signal distribution with the respective mean lifetime. The input lifetimes of the four samples were not revealed until the fit results were returned thus allowing a blind test of the fit. The fit was applied to each of the four sets and table 5.3 shows the fit results together with the input values used for the generation of the samples. The results agree well with the input lifetime.

\begin{tabular}{|c|c|}
\hline Fit Result $[\boldsymbol{\mu m}]$ & Input Value $[\boldsymbol{\mu m}]$ \\
\hline $375.2 \pm 5.8$ & 380 \\
\hline $444.2 \pm 7.1$ & 450 \\
\hline $300.4 \pm 4.5$ & 300 \\
\hline $485.7 \pm 7.9$ & 500 \\
\hline
\end{tabular}

Table 5.3: Results of a blind fit to four sets of simulated signal samples whose lifetime was unknown at the time of the fit together with the statistical errors.

A second blind test was used to determine whether the fit reproduces the input lifetime in the presence of background. Eight samples with unknown lifetime were 
generated, consisting each of a sample of signal and background and a backgroundonly sample. Again the input lifetime was not known at the time of the fit. The results are shown in table 5.4. Again the input values, revealed after completion of the fit, are reproduced within the statistical error of the fit.

\begin{tabular}{|c|c|}
\hline Fit Result $[\boldsymbol{\mu m}]$ & Input Value $[\boldsymbol{\mu m}]$ \\
\hline $457.4 \pm 18.1$ & 470 \\
\hline $374.0 \pm 15.4$ & 360 \\
\hline $421.5 \pm 17.0$ & 410 \\
\hline $315.9 \pm 13.3$ & 310 \\
\hline $518.4 \pm 19.8$ & 520 \\
\hline $303.4 \pm 12.8$ & 300 \\
\hline $456.6 \pm 18.1$ & 460 \\
\hline $396.6 \pm 16.1$ & 380 \\
\hline
\end{tabular}

Table 5.4: Results of a blind fit to eight sets of simulated samples whose lifetime was unknown at the time of the fit. Each set consists of a sample containing a mix of signal and background events and a sample containing only background events. The errors on the fit results are statistical only.

As a further test 1000 toy experiments were generated to test the signal fit. Each experiment contains the estimated amount of true signal events seen in data. The input values to the fit, $c \tau^{\prime}$ and $\sigma_{c \tau^{\prime}}$, were randomly drawn from the signal distribution with a mean decay length of $368 \mu \mathrm{m}$. For a satisfactory fit the average of the $c \tau$ values returned by the 1000 separate fits should lie within the statistical error of this average, $\sigma / \sqrt{1000}$, and the pull distribution $\left(c \tau_{\text {input }}-c \tau_{f i t}\right) / \sigma_{c \tau}$ should be centred around zero and have a width of one, for the error returned by the fit to be considered correct. Figure 5.9 (a) and (b) show the mean and the pull distribution of the 1000 toy experiments. The mean is consistent with the input lifetime and the mean and width of the pull distribution are consistent with zero and one respectively.

The $c \tau$ pull distribution for the signal was then tested in the presence of background, again using 1000 toy experiments. The amount of background events and the signal-to-background ratio were taken from the prospective number of events expected from data. Figure 5.9 (c) and (d) show the mean of the resulting $c \tau$ values and the pull distribution for this experiment. Again the fit is satisfactory with a mean of $368.2 \pm 1.6 \mu \mathrm{m}$ and a pull distribution whose mean and width are consistent with zero and one respectively. 


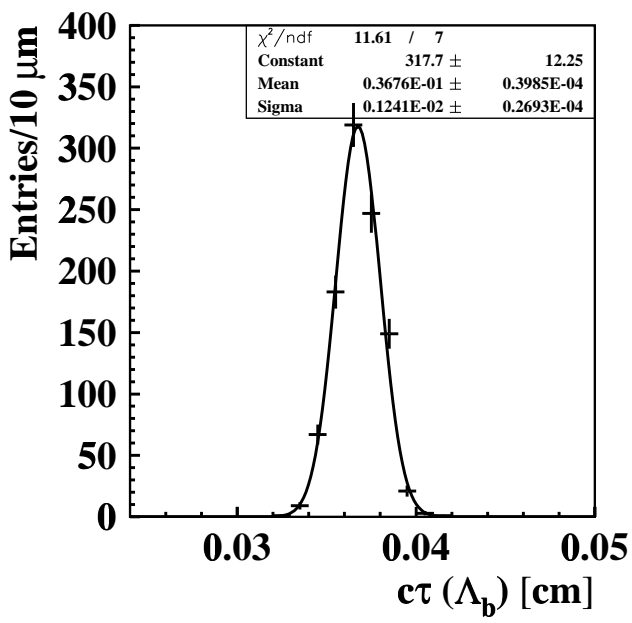

(a)

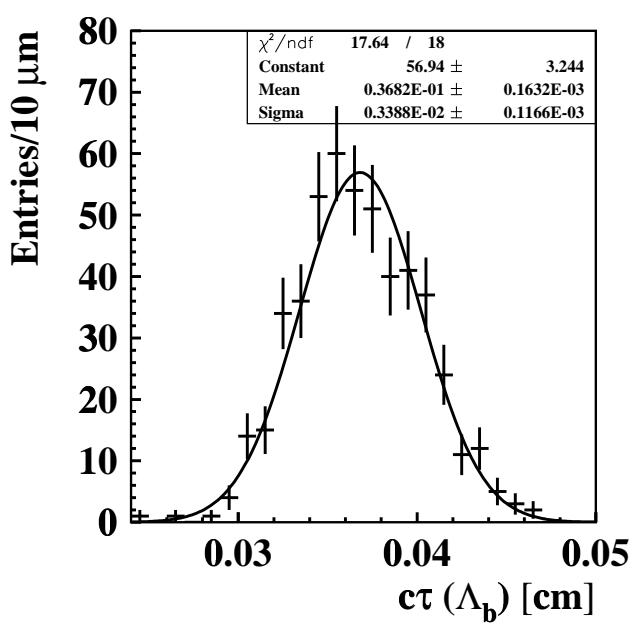

(c)

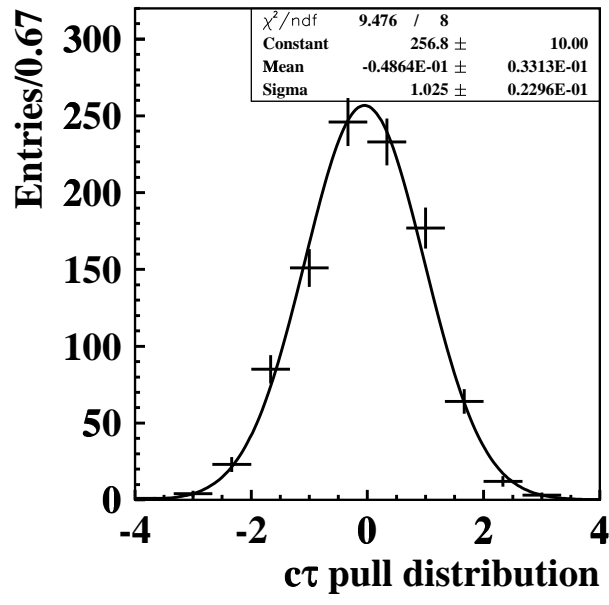

(b)

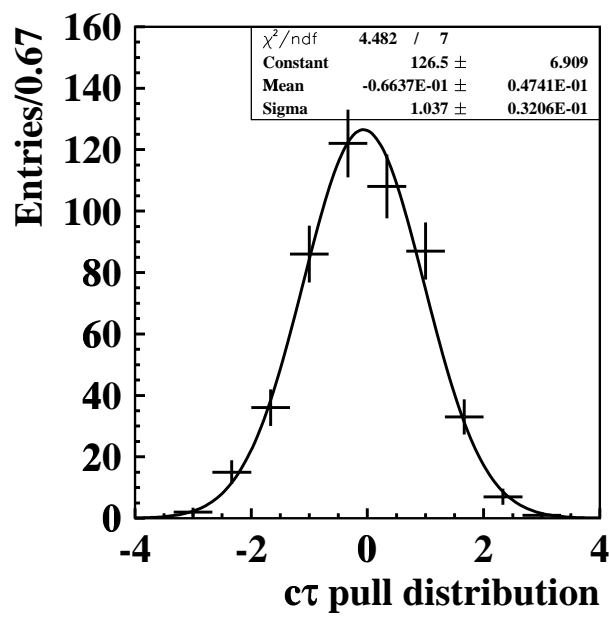

(d)

Figure 5.9: Figure (a) and (b) show the mean of the $c \tau$ values and the pull distribution obtained by fitting 1000 toy experiments generated according to the signal function. Figure (c) and (d) show the same quantities as (a) and (b), this time for a fit to 1000 toy experiments where signal events were generated in the presence of background.

\subsubsection{Fit to the Realistic Simulation}

As a final test the fit was applied to the realistic simulation which only contains signal events. The K-factor and the $c \tau$ distribution which are extracted for the fit from the parametric simulation are shown in figure 5.10 (a) and (b). The fit to the complete sample of 4486 events returns a lifetime of $374.6 \pm 6.0 \mu \mathrm{m}$. Subsequently the sample is divided into smaller samples of about the size of the true signal sample 


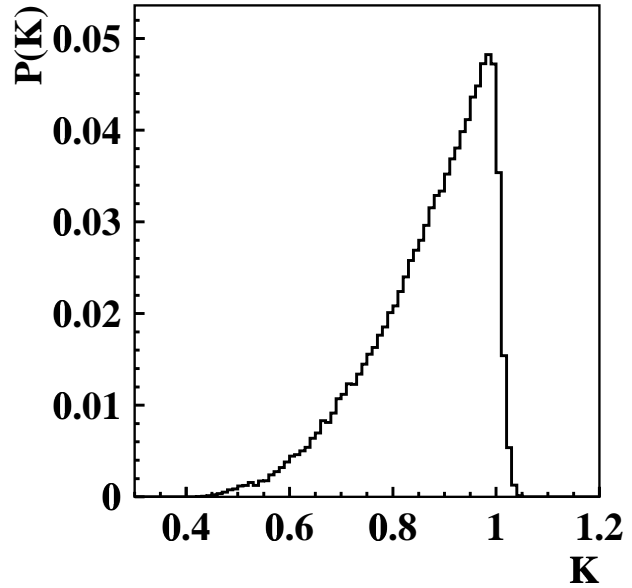

(a)

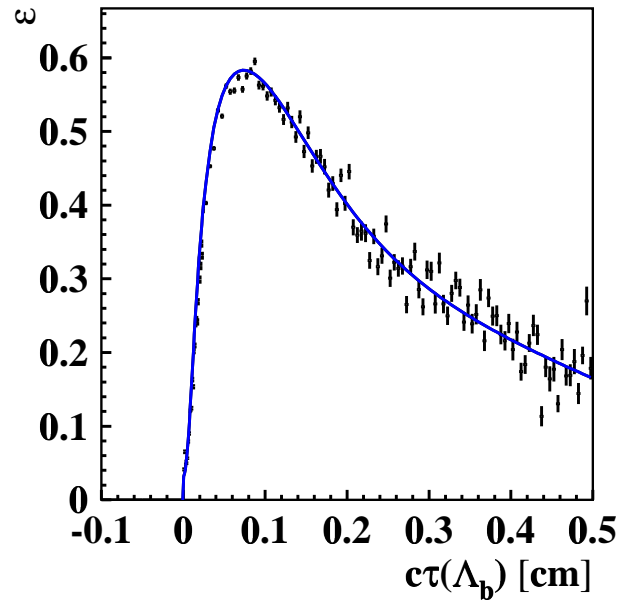

(b)

Figure 5.10: Figure (a) and (b) show the K-factor and $c T$ efficiency distribution used for the fit to the realistic simulation.

from data. This results in four samples of 1000 events and one sample containing the remaining 486 events. The single fit results are summarised in table 5.5. The weighted average of these five results is $373.7 \mu \mathrm{m}$ which agrees well with the result from the single fit.

\begin{tabular}{|c|c|}
\hline Number of Events & Fit Result [ $\boldsymbol{\mu m}]$ \\
\hline 1000 & $384.5 \pm 13.0$ \\
\hline 1000 & $376.9 \pm 12.7$ \\
\hline 1000 & $358.8 \pm 12.0$ \\
\hline 1000 & $372.3 \pm 12.5$ \\
\hline 486 & $387.6 \pm 18.9$ \\
\hline
\end{tabular}

Table 5.5: Results of the fits to five sub-samples of the realistic simulation.

The systematic uncertainties associated with the lifetime measurement are discussed in chapter 6 and the results of the $\Lambda_{b}$ lifetime measurement are presented in chapter 7 . 


\section{Chapter 6}

\section{Systematic Effects}

Most of the input parameters to the lifetime fit are known only up to a certain accuracy. This uncertainty on the input parameters leads to a systematic uncertainty on the lifetime of the $\Lambda_{b}$ as extracted by the lifetime fit. This chapter describes the various systematic errors assigned by repeating the lifetime fit with varied input parameters.

The first set of parameters considered are those that enter the fit via the determination of the distribution of the K-factor and the $c \tau$ efficiency. The K-factor and the $c \tau$ efficiency are extracted from the parametric simulation and are, apart from the data itself, the most important inputs to the lifetime fit. To evaluate the effect of their uncertainty on the systematic error of the lifetime fit, 1000 toy Monte Carlos samples were generated with 1000 events each. A toy Monte Carlo is used because it allows the fast generation of a large number of samples. It is referred to as a "toy" $\mathrm{MC}$ because only the input parameters for the fit, $c \tau^{\prime}$ and $\sigma\left(c \tau^{\prime}\right)$, are generated, while omitting the generation of particles with associated momenta and decay vertices. For this purpose events are randomly drawn from an exponential distribution with the appropriate lifetime. The K-factor, the $c \tau$ efficiency and the detector resolution are then applied to those events. The inputs to those toy Monte Carlo samples are the original K-factor and $c \tau$ efficiency distributions (see figures 5.2 (a), 5.6 (b) and figure 7.1 (a) and (b)) which are used to determine the lifetime of the $\Lambda_{b}$ in data.

After the generation of the toy $\mathrm{MC}$ samples, the input parameters of the parametric simulation (see chapter 5) were varied, which results in new distributions for 
the K-factor and the $c \tau$ efficiency. The toy Monte Carlo samples were then fitted with these new curves and a shift in the result of the lifetime fit is assigned as a systematic error. The variation of the input parameters as well as the shift observed are described in the following. The resulting systematic errors on $c \tau$ are summarised in table 6.2.

\section{Uncertainty on the SVT Tracking Efficiency}

The SVT tracking efficiency is parametrised by the function

$$
\varepsilon_{\mathrm{SVT}}(\mathrm{d} 0)=0.5 \cdot \mathrm{P} 1 \cdot \operatorname{erfc}((|\mathrm{d} 0|-\mathrm{P} 2) / \mathrm{P} 3) .
$$

For data the parameters are $\mathrm{P} 1=0.697, \mathrm{P} 2=0.177 \mathrm{~cm}$ and $\mathrm{P} 3=0.075 \mathrm{~cm}$. This curve is reasonably flat over the range $0 \leq \mathrm{d} 0[\mathrm{~cm}] \leq 0.1$ (see figure 5.4). However even an entirely flat efficiency curve has to be accounted for in the extraction of the $\mathrm{K}$-factor and the $c \tau$ efficiency. To cover the variation of the SVT efficiency owing to different SVT configurations the plateau efficiency is varied by \pm 0.1 , i.e. the $\mathrm{K}$-factor and the $c \tau$ efficiency curve are extracted using the parameters $\mathrm{P} 1=0.597$ and $\mathrm{P} 1=0.797$ when applying the SVT tracking efficiency, and the parameters P2 and P3 are varied by $\pm 0.025 \mathrm{~cm}$. Figure 6.1 shows the SVT tracking efficiency curve (a) and the resulting $c \tau$ efficiency curve (b) for the three different values of P1, P2 and P3. The change in the K-factor distribution is hardly visible. Applying the lifetime fit to the toy experiments (which were generated with $\mathrm{P} 1=0.697$ ), using the K-factor and $c \tau$ efficiency curve obtained with $\mathrm{P} 1=0.797$, results in a three micron longer lifetime; using $\mathrm{P} 1=0.597$ the fit returns a three micron shorter lifetime. Changing P2 by minus and plus $0.025 \mathrm{~cm}$ results in a shift of $+2 \mu \mathrm{m}$ and $-1 \mu \mathrm{m}$ respectively. Finally using the K-factor and $c \tau$ efficiency curve obtained with $\mathrm{P} 3=0.050 \mathrm{~cm}$ results in a shift of $-1 \mu \mathrm{m}$ and using $\mathrm{P} 3=0.100 \mathrm{~cm}$ the fit result is shifted by $+2 \mu \mathrm{m}$. Thus a total systematic error of ${ }_{-3}^{+4} \mu \mathrm{m}$ is assigned due to the uncertainty on the SVT tracking efficiency. 

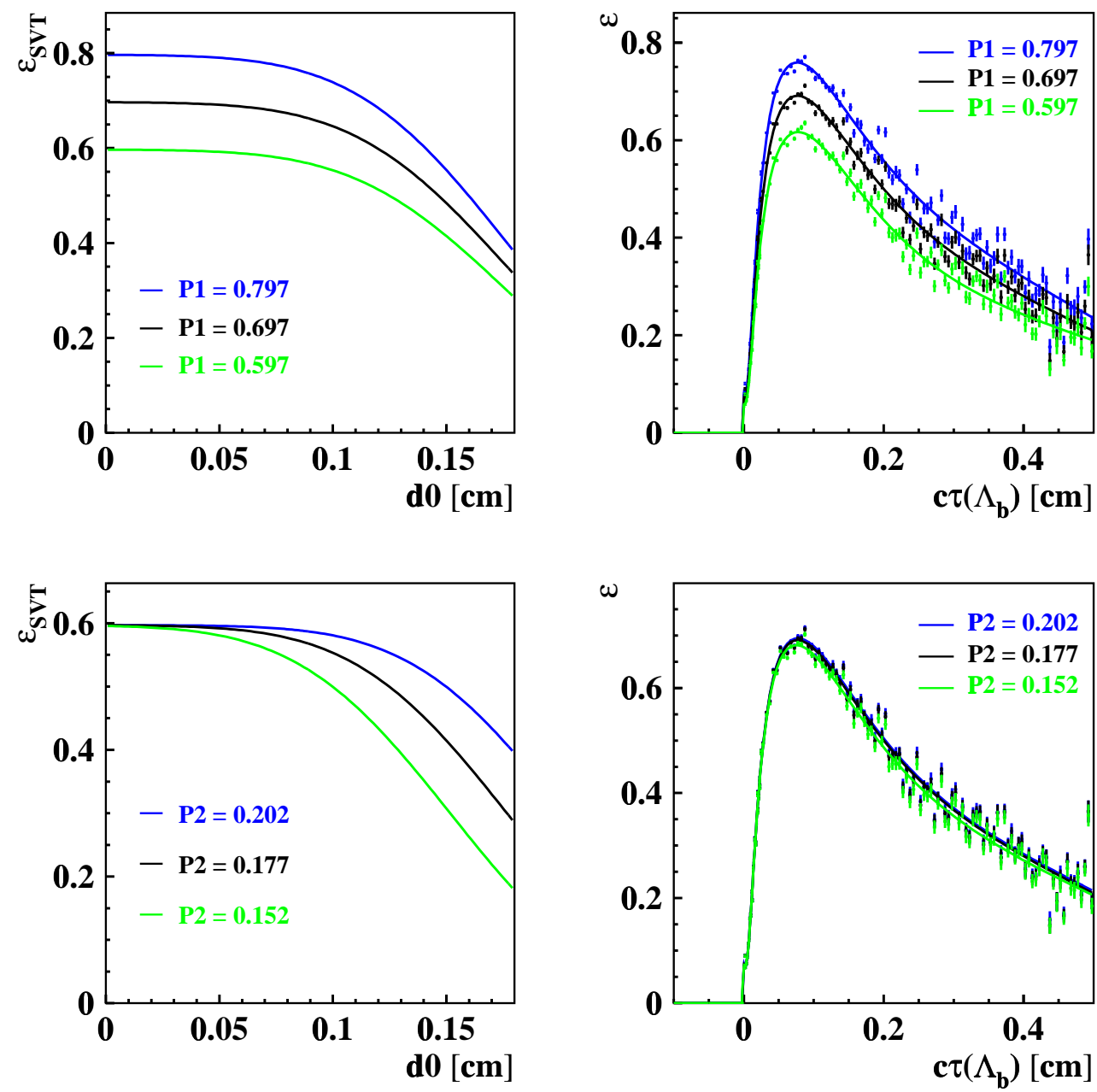

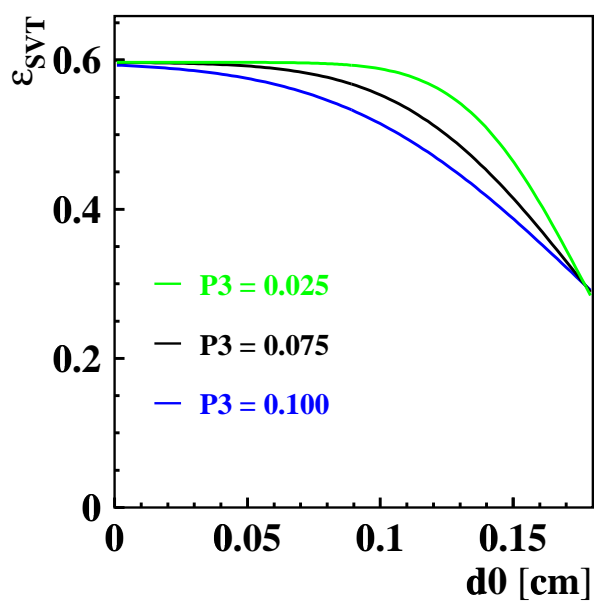

(a)

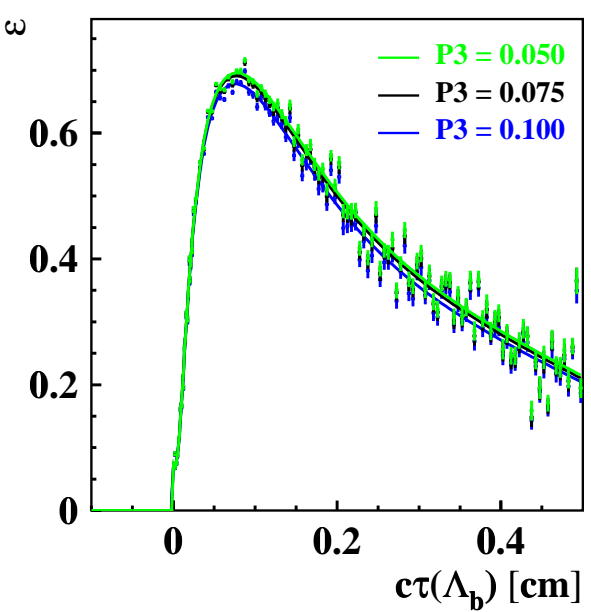

(b)

Figure 6.1: SVT tracking efficiency (a) and $c \tau$ efficiency (b) for three different values of P1 (top), P2 (middle) and P3 (bottom). 


\section{Uncertainty on $\sigma_{S V T}$ and $\sigma_{S V X}$}

The error on the resolution of the impact parameter as measured by the SVT $\left(\sigma_{S V T}\right)$ and the SVX $\left(\sigma_{S V X}\right)$ as well as the correlation between the two are treated next. Again the K-factor and $c \tau$ efficiency curve are extracted varying the three above variables independently. The resolution of the impact parameter is changed by $\pm 10 \mu \mathrm{m}$ and the correlation coefficient (see equation 5.1) is changed by \pm 0.1 . This yields the following shifts in the lifetime obtained from the fit of the toy Monte Carlo samples. Enhancement of $\sigma_{S V T}$ to 0.067 yields a one micron longer lifetime, decreasing the value to 0.047 yields a three micron shorter lifetime.

An increase of $\sigma_{S V X}$ to 0.055 results in a five micron longer lifetime, while the decrease to 0.035 results in a seven micron shorter lifetime. Changing the correlation coefficient $\rho$ from 0.8 to 0.7 and 0.9 yields a shift in the lifetime of minus and plus five micron respectively. This results in a total systematic error from the uncertainty on the impact parameter resolution of ${ }_{-9}^{+7} \mu \mathrm{m}$.

\section{Shift of the Impact Parameter}

When the impact parameter obtained from the realistic simulation was compared with the true impact parameter calculated from the generator level information on vertices and momenta, a shift at the 0-10 micron level was observed. It is believed that this shift is caused by the fact that the silicon ladder bow is not simulated in the detector geometry, while the reconstruction code assumes a bow is present and thus corrects for it (see section 5.1.2). This shift needs to be considered for the evaluation of the systematic error on the fit to the realistic simulation. However, as it cannot be excluded that the shift has an alternate cause and could thus also be present in data, the systematic uncertainty is also included for the lifetime fit to the data. To evaluate the effect of this uncertainty on the lifetime result, the impact parameter is shifted by $\pm 10 \mu \mathrm{m}$ to obtain new input distributions for the fit. Repeating the fit to the sample of toy experiments as well as to the realistic simulation results consistently in a shift of $+15 \mu \mathrm{m}$ for the reduced impact parameter and $-15 \mu \mathrm{m}$ for a shift of $+10 \mu \mathrm{m}$. An uncertainty of $\pm 15 \mu \mathrm{m}$ is assigned. 


\section{Input Lifetime}

The current value of the mean lifetime of the $\Lambda_{b}$ baryon quoted by the Particle Data Group [1] is $1.229 \pm 0.08010^{-12} \mathrm{~s}$ which translates to a mean decay length of $368 \pm 24 \mu \mathrm{m}$. The parametric simulation is generated with a mean decay length of $368 \mu \mathrm{m}$ for the $\Lambda_{b}$ baryon. To evaluate the influence of the correct input lifetime to the lifetime fit, two new samples of the parametric simulation were generated with a mean decay length of $343 \mu \mathrm{m}$ and $393 \mu \mathrm{m}$, each consisting of ten files with approximately 12000 events each after the selection cuts. These samples are then fitted using the K-factor and $c \tau$ distribution as obtained from the sample with the mean decay length of $368 \mu \mathrm{m}$. As expected the change of $\pm 25 \mu \mathrm{m}$ has very little impact on the final fit result. An error of $\pm 2 \mu \mathrm{m}$ is assigned.

\section{Selection Cuts}

The distributions of the selection variables for data and the two Monte Carlo samples showed reasonable agreement within the error on the data distributions (see Appendix A), although these errors are large due to the large amount of background events present under the signal peak in the $p K \pi$ invariant mass plot. If a data distribution of a selection variable differs from the one for the simulated events then the selection cut can introduce a bias to the lifetime measurement. To estimate the systematic uncertainty associated with this possible bias new K-factor and $c \tau$ efficiency curves were obtained with corrected cut values. The lifetime fit to the standard data sample was then repeated and the new lifetime value was compared to the standard result.

The following variables are considered: $\mathrm{p}_{\mathrm{T}} \mathrm{p} / \mathrm{p}_{\mathrm{T}} \pi, \mathrm{p}_{\mathrm{T}} \mathrm{p}, \mathrm{p}_{\mathrm{T}} \mathrm{K}, \mathrm{p}_{\mathrm{T}} \Lambda_{\mathrm{c}}$ and $\mathrm{m}\left(1 \Lambda_{\mathrm{c}}\right)$. Looking at the data-MC comparison the fraction of events cut out by the requirement $\mathrm{p}_{\mathrm{T}} \mathrm{p} / \mathrm{p}_{\mathrm{T}} \pi>1.3$ is slightly higher in the parametric simulation than in data. The cut for the determination of the K-factor and $c \tau$ efficiency curves is thus lowered to 1.2 which results in a $2 \mu \mathrm{m}$ higher fit result.

The distribution of $\mathrm{p}_{\mathrm{T}} \mathrm{p}$ shows good agreement between data and MC. Arguably again slightly more events are cut out in the parametric simulation. Moving the cut on $\mathrm{p}_{\mathrm{T}} \mathrm{p}$ from $2 \mathrm{GeV} / \mathrm{c}$ to $1.8 \mathrm{GeV} / \mathrm{c}$ results in a shift of $-1 \mu \mathrm{m}$. 
For $\mathrm{p}_{\mathrm{T}} \mathrm{K}$ the distribution of the parametric simulation lies slightly below the distribution extracted from data - for values lower and around the cut value. The cut is thus tightened from $0.9 \mathrm{GeV} / \mathrm{c}$ to $1.1 \mathrm{GeV} / \mathrm{c}$ which has no significant effect on the fit result.

For the variable $\mathrm{p}_{\mathrm{T}} \Lambda_{\mathrm{c}}$ the agreement is again good. Below the cut value set at $5 \mathrm{GeV} / \mathrm{c}$ the parametric simulation has slightly fewer events then the distribution from data so that the effect of tightening the cut to $5.5 \mathrm{GeV} / \mathrm{c}$ is investigated. This changes the fit result by $-1 \mu \mathrm{m}$.

The last variable considered is $\mathrm{m}\left(1 \Lambda_{\mathrm{c}}\right)$, which somewhat differs in shape between the simulated events and the data. The difference around the upper cut value is negligible, and the difference below the lower cut value is small. The curve of the parametric simulation lies here slightly below the curve for data so that the cut is tightened from $3.6 \mathrm{GeV} / \mathrm{c}^{2}$ to $3.8 \mathrm{GeV} / \mathrm{c}^{2}$. This yields a $5 \mu \mathrm{m}$ larger result in the lifetime fit.

Additionally it is investigated whether the cut values for the important selection variables $c \tau\left(\Lambda_{c}\right)$ and $L_{x y}\left(p . v .-\Lambda_{c}\right)$ were carefully chosen such that the amount of signal rejected should be negligible. As these cuts reject a large amount of background events the effect of dropping those cuts is tested on the realistic simulation. To estimate the systematic uncertainty associated with a possible bias introduced through the above cuts the event selection for the realistic simulation is repeated by dropping the above cuts one by one. The fit is then repeated with the standard K-factor and $c \tau$ efficiency curves and the new lifetime value is compared to the standard result. Dropping the cuts on those variables should have a negligible effect as compared to the statistical error $(6 \mu \mathrm{m})$ on the fit. Dropping the cut on $L_{x y}\left(p . v .-\Lambda_{c}\right)$ results in a shift by $-1 \mu \mathrm{m}$. Dropping the cut on $c \tau\left(\Lambda_{c}\right)$ yields a result shifted by just under $1 \mu \mathrm{m}$. If only the upper cut, $c \tau\left(\Lambda_{c}\right)<0.035$ is released, a shift of $-1 \mu \mathrm{m}$ is observed, while dropping the lower cut, $c \tau\left(\Lambda_{c}\right)>-0.015$ yields a $2 \mu \mathrm{m}$ larger result.

The only sizeable effect amongst the above is the tightening of the cut on $\mathrm{m}\left(1 \Lambda_{\mathrm{c}}\right)$. Thus a systematic uncertainty due to the selection cuts of $+5 \mu \mathrm{m}$ is assigned. 


\section{Fragmentation}

The Monte Carlo simulations used in this thesis use the Peterson fragmentation function to determine what longitudinal momentum fraction $z$ the $\Lambda_{b}$ baryon carries as compared to the primordial heavy b quark it originates from (see section 2.4.3). It is investigated here whether and to what extent the result of the lifetime fit depends on the correct Peterson fragmentation parameter $\epsilon$. The parametric simulation used for the final fit is generated with an epsilon of 0.006. To evaluate a possible systematic error from the uncertainty on this value three Monte Carlo samples of the parametric simulation are generated with the values $0.0025,0.0095$ and 0.024 for epsilon. The first two values, 0.0025 and 0.0095 , are taken from a study by P.S. Wells [62] that determined reasonable values for the systematic variation of fragmentation parameters for $\mathrm{b}$ physics analyses. The additional use of the value 0.024 is motivated by the following. The epsilon value for b quarks was measured at LEP by the ALEPH Collaboration by measuring the energy distribution of $B^{0}$ and $B^{ \pm}$mesons [63]. Similarly the functional form of the Peterson fragmentation function was originally derived for mesons, i.e. for the case that a single quark combines with the heavy b quark (see section 2.4.3). It is not established whether the measured value for epsilon remains correct in the case of baryon creation while maintaining the original functional form of the fragmentation function. A baryon contains three quarks or three anti-quarks; for the generation of a $\Lambda_{b}$ the b quark has to combine with two light quarks, an up and a down quark, as opposed to the meson case where only one anti-quark has to be found to combine with the existing quark. The energy difference of the system before and after the fragmentation is determined below for the baryon case following the meson case from section 2.4.3. Two approximations are made here for the estimation of the effect of a change from mesons to baryons:

- $m_{u}=m_{d}$, i.e. the two quarks that combine with the b-quark are assumed to have equal masses

- the momenta of the two remaining light anti-quarks are of the same size. 
With these approximations the energy difference for a heavy quark $Q$ with momentum $P$ that fragments into a heavy hadron $H=Q q q^{\prime}$ with momentum $z P$ and the remaining light quarks $\bar{q} \bar{q}^{\prime}$ is

$$
\Delta E=E_{Q}-E_{H}-2 E_{q}=\sqrt{m_{Q}^{2}+P^{2}}-\sqrt{m_{H}^{2}+z^{2} P^{2}}-\sqrt{4 m_{q}^{2}+(1-z)^{2} P^{2}} .
$$

Assuming as previously that $m_{Q} \approx m_{H}$ this results in

$$
\begin{aligned}
\Delta E=P\left[\sqrt{\frac{m_{Q}^{2}}{P^{2}}+1}\right. & \left.-\sqrt{\frac{m_{Q}^{2}}{P^{2}}+z^{2}}-2 \sqrt{\frac{m_{q}^{2}}{P^{2}}+\frac{1}{4}(1-z)^{2}}\right]= \\
P & {\left[\sqrt{\frac{m_{Q}^{2}}{P^{2}}+1}-\sqrt{\frac{m_{Q}^{2}}{P^{2}}+z^{2}}-2 \sqrt{\frac{m_{Q}^{2}}{P^{2}} \frac{m_{q}^{2}}{m_{Q}^{2}}+\frac{1}{4}(1-z)^{2}}\right] . }
\end{aligned}
$$

Expanding around $x=m_{Q}^{2} / P^{2}$ at $x=0$ gives for $P \gg m_{Q}$

$$
\Delta E=\frac{m_{Q}^{2}}{2 P}\left[1-\frac{1}{z}-4 \frac{\epsilon_{Q}}{1-z}\right]
$$

compared to the energy difference

$$
\Delta E=\frac{m_{Q}^{2}}{2 P}\left[1-\frac{1}{z}-\frac{\epsilon_{Q}}{1-z}\right]
$$

obtained for the meson case. So instead of changing the code for MC generation by replacing $\epsilon$ by $4 \epsilon$, the same code can be used, but with $\epsilon=4 \times 0.006=0.024$. (The same result is obtained by regarding the generation of a di-quark with $m=2$ $m_{q}$ ). Figure 6.2 shows the Peterson fragmentation function for all values used in the $\mathrm{MC}$ generation. Fitting the different samples while using the original K-factor and $c \tau$ distribution obtained with $\epsilon=0.006$ leads to only slight changes in the lifetime result. Even for the sample with $\epsilon=0.024$ a shift of only $1.5 \mu \mathrm{m}$ is observed and this effect is thus considered negligible. The change in $\epsilon$ does however result in a change of the selection efficiency. This is caused by the fact that the higher $\epsilon$ is, the lower the momentum of the new hadron is and fewer events pass the cuts on the transverse momenta of the lepton and the $\Lambda_{c}$. 


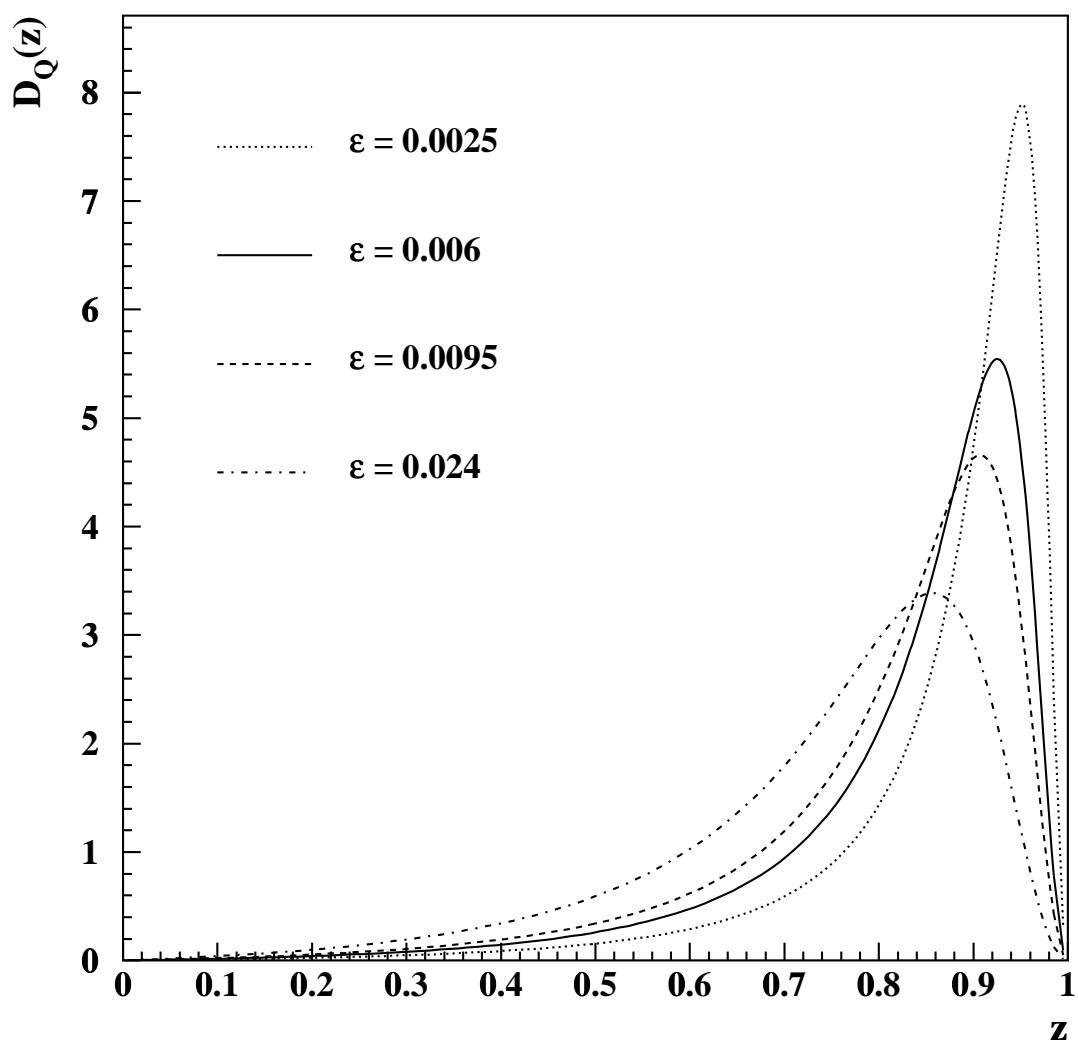

Figure 6.2: Peterson fragmentation function for four different values of epsilon: $\epsilon=0.0025,0.006,0.0095$ and 0.024 .

\section{$\Lambda_{b}$ Polarisation}

The polarisation of a particle is considered as an asymmetry in its spin orientation. In the heavy quark limit the initial polarisation of a $\mathrm{b}$ quark is expected to be transferred to a ground baryonic state directly produced in the hadronisation of the b quark. At the Large Electron Positron Collider LEP the polarisation of $\Lambda_{b}$ baryons from hadronic $\mathrm{Z}$ decays was measured by the ALEPH, DELPHI and OPAL collaborations [64-66]. The results of these measurements are summarised in table 6.1. (Considering that the average polarisation of $\mathrm{b}$ quarks from $\mathrm{Z}$ decays in the SM is $\left\langle P_{L}^{b}\right\rangle=-0.94$, the measured values for $\Lambda_{b}$ polarisation hint towards the importance of depolarisation mechanisms such as indirect $\Lambda_{b}$ production through heavier states.) 


\begin{tabular}{|c|c|}
\hline Collaboration & Result \\
\hline ALEPH & $-0.23_{-0.20}^{+0.24}$ (stat.) ${ }_{-0.07}^{+0.08}$ (syst.) $[64]$ \\
\hline DELPHI & $-0.49_{-0.30}^{+0.32}$ (stat.) \pm 0.17 (syst.) $[65]$ \\
\hline OPAL & $-0.56_{-0.13}^{+0.20}$ (stat.) \pm 0.09 (syst.) $[66]$ \\
\hline
\end{tabular}

Table 6.1: Measurements of the $\Lambda_{b}$ polarisation at LEP.

At hadron colliders the $\Lambda_{b}$ polarisation has not been measured and this lack of knowledge may give rise to an uncertainty on the lifetime measurement. The knowledge of the polarisation $P$ can be potentially important for an analysis due to the different angular spectrum of leptons emitted from polarised $\Lambda_{b}$ baryons. If the $\Lambda_{b}$ is fully polarised, i.e. if its spin and momentum vector are parallel $(P=+1)$ or anti-parallel $(P=-1)$, the lepton (in the $\Lambda_{b}$ rest frame) is preferentially emitted in opposite direction to the spin of the $\Lambda_{b}$ while the anti-neutrino is preferentially emitted in spin direction, assuming that the decay proceeds via the usual left-handed interaction. For the case of the fully polarised anti-baryon, $\bar{\Lambda}_{b}$, the directions are reversed yielding a neutrino emitted anti-parallel to the spin of the $\Lambda_{b}$ and a lepton emitted parallel to it (again in the $\Lambda_{b}$ rest frame). It is therefore possible that the $\Lambda_{b}$ polarisation affects the K-factor and $c \tau$ efficiency distribution.

The simulated events used in this analysis contain unpolarised $\Lambda_{b}$ baryons, which means that the cosine of the angle between the momentum of the $\Lambda_{b}$ in the laboratory frame and the momentum of the lepton in the rest frame of the $\Lambda_{b}, \cos \theta$, should be distributed uniformly. For polarised $\Lambda_{b}$ 's on the other hand there should be an excess of events at $\cos \theta= \pm 1$ depending on whether the spin of the $\Lambda_{b}$ is anti-parallel or parallel to its direction of motion. Preselection cuts applied during the generation of the parametric simulation to filter out the kinematically relevant events may influence the distribution of $\cos \theta$. As such a sample of the parametric simulation is generated without the application of these filter cuts, to test whether the simulated events contain unpolarised $\Lambda_{b}$ 's. In addition to dropping the filter cuts, the generation range of the $\mathrm{p}_{\mathrm{T}}$ of the $\mathrm{b}$ quarks is extended from $\mathrm{p}_{\mathrm{T}} \geq 6 \mathrm{GeV} / \mathrm{c}$ to $\mathrm{p}_{\mathrm{T}} \geq 0.2 \mathrm{GeV} / \mathrm{c}$, while the $\eta$ range is extended from $\left|\eta_{b}\right| \leq 2$ to $\left|\eta_{b}\right| \leq 5$. Figure 6.3 (a) shows the resulting distribution of $\cos \theta$ which is indeed flat. Figure 6.3 (b) shows the same distribution obtained from the standard sample of the parametric simulation, i.e. using $\mathrm{p}_{\mathrm{T}} \mathrm{b}>6 \mathrm{GeV} / \mathrm{c},\left|\eta_{b}\right|<2$ as well as the filter cuts 


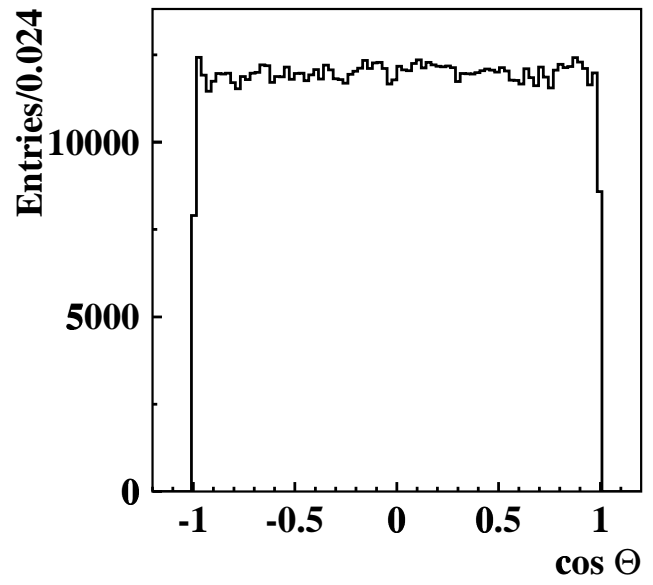

(a) no cuts

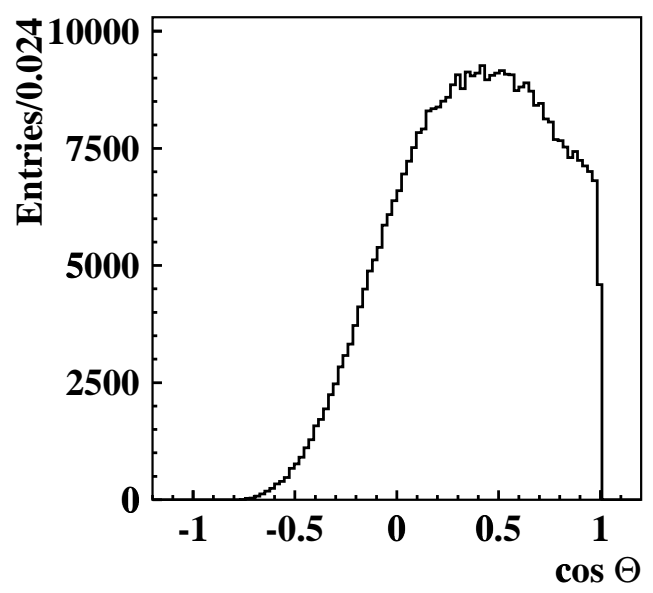

(c) trigger cuts

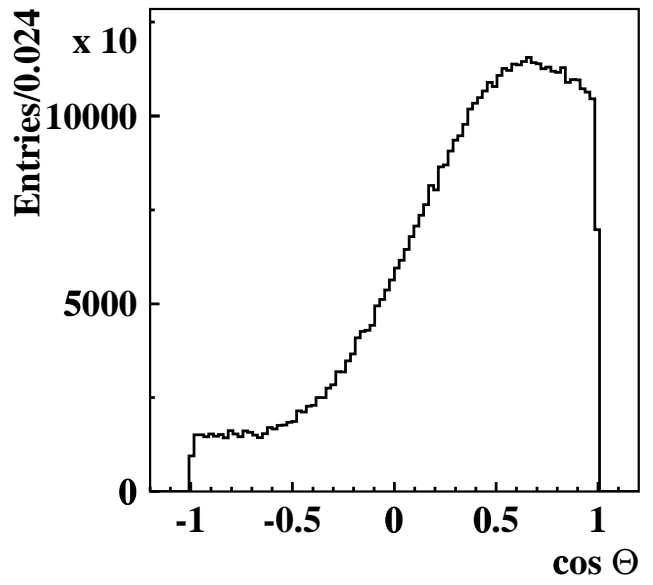

(b) filter cuts

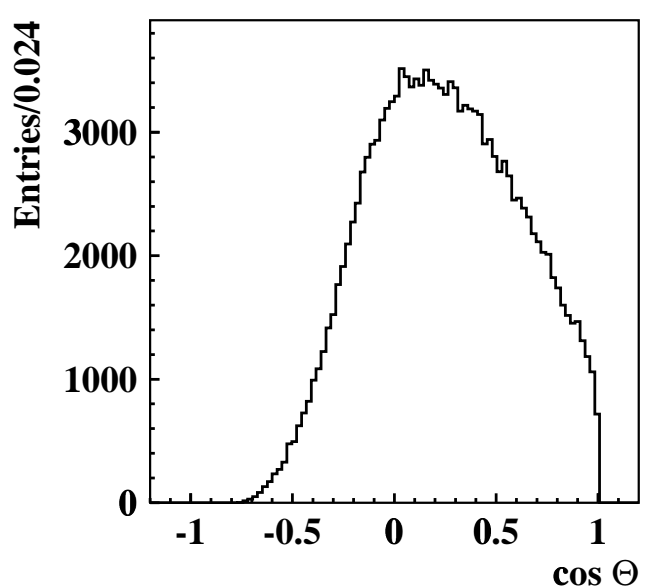

(d) all selection cuts

Figure 6.3: Distribution of $\cos \theta$ after the application of various cuts explained in the text. The angle $\theta$ is defined here as the angle between the momentum of the $\Lambda_{b}$ in the laboratory frame and the momentum of the lepton in the rest frame of the $\Lambda_{b}$.

$\mathrm{p}_{\mathrm{T}} \Lambda_{\mathrm{c}}>2 \mathrm{GeV} / \mathrm{c}, \mathrm{p}_{\mathrm{T}}(\mathrm{l})>3.5 \mathrm{GeV} / \mathrm{c},\left|\eta_{\Lambda_{c}}\right|<1.2$ and $\left|\eta_{\mu}\right|<0.8$ which highlights the influence of the selection cuts on this distribution. Figure 6.3 (c) shows the distribution obtained after application of all trigger cuts and figure 6.3 (d) finally shows the distribution after all cuts applied in the analysis. Figure 6.3 (d) shows that the selection cuts prefer events where leptons as well as anti-leptons (in the $\Lambda_{b}$ rest frame) are emitted in direction of the $\Lambda_{b}$ in the laboratory frame.

The Monte Carlo generator used here has no inbuilt option for adjusting the polarisation of the $\Lambda_{b}$ so that the events have to be re-weighted to assign a systematic 
error due to the absence of knowledge on the true polarisation of the $\Lambda_{b}$. The weight, $1 / \pi \cdot(1 \pm \cos \theta)$, is applied on an event-by-event basis to the input distributions to the fit. The factor $1 / \pi$ originates form the normalisation condition

$$
\int_{0}^{\pi}(1-\cos \theta) d \theta=1
$$

and the sign depends on the sign of the $\Lambda_{b}$ polarisation as well as on whether the decay is from a $\Lambda_{b}$ or $\bar{\Lambda}_{b}$.

For the case of a $\Lambda_{b}$ with polarisation +1 the weight is $1 / \pi \cdot(1-\cos \theta)$ : the decay of a $\Lambda_{b}$ results in a lepton and an anti-neutrino. That means in the case of polarised $\Lambda_{b}$ baryons that the lepton, as seen in the $\Lambda_{b}$ rest frame, is emitted preferentially anti-parallel to the spin of the $\Lambda_{b}$ baryon. For polarisation +1 the spin and momentum of the $\Lambda_{b}$ are parallel so that the preferred angle $\theta$ is $180^{\circ}$ and events with $\theta=180^{\circ}$ should have the highest weight, while events with $\theta=0^{\circ}$ should have the lowest weight. Following this argument for the case of $\mathrm{P}=-1$ the weight $1 / \pi \cdot(1+\cos \theta)$ is obtained. For decays of the anti baryon, $\bar{\Lambda}_{b}$, the signs reverse.

In data the number of events from baryon and anti-baryon decay are approximately equal. Figure 6.4 shows the $p K \pi$ invariant mass distribution for both cases. This similarity of selection efficiencies for $\Lambda_{b}$ and $\bar{\Lambda}_{b}$ hints towards relatively small polarisation effects since the selection cuts are biased towards selecting events where the leptons/anti-leptons (in the $\Lambda_{b}$ restframe) are observed in the direction of the $\Lambda_{b} / \bar{\Lambda}_{b}$, which is in contrast to the decay signature expected for a strongly polarised sample. To evaluate the systematic error the conservative case of fully polarised $\Lambda_{b}$ 's is considered. The new $\mathrm{K}$-factor and $c \tau$ efficiency curves are obtained by alternately applying the weight with positive and negative sign in front of the $\cos \theta$ term to account for the fact that an approximately equal number of events from $\Lambda_{b}$ and $\bar{\Lambda}_{b}$ decay are observed in data. Refitting the sample of the parametric simulation yields a $3 \mu \mathrm{m}$ shorter result. An error of $\pm 3 \mu \mathrm{m}$ is assigned to the lifetime measurement.

The parameters varied above concerned the input parameters to the parametric simulation and the errors that arise due to their uncertainty. Additionally there are a few parameters that enter the lifetime fit directly and those are described below. 


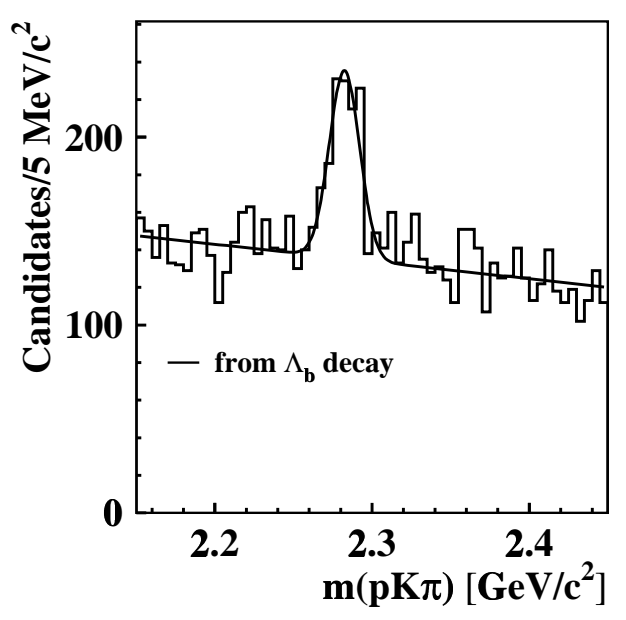

(a)

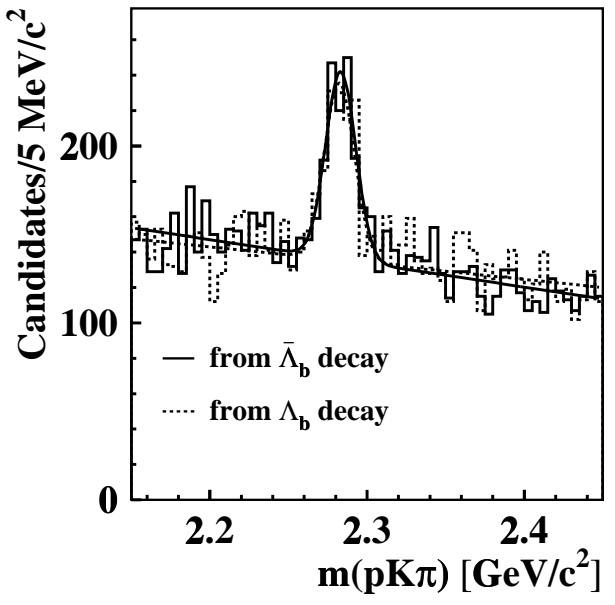

(b)

Figure 6.4: Figure (a) shows the $p K \pi$ invariant mass plots for events containing a muon, i.e. candidates from $\Lambda_{b}$ decays. Figure (b) shows the same for events containing an anti-muon, i.e. candidates from $\bar{\Lambda}_{b}$ decays with the distribution shown in (a) overlaid.

\section{Error Scale Factor}

The error on the measurement of the decay length $L$ projected into the $x y$-plane $\sigma\left(L_{x y}\right)$ is extracted from the vertex fit and is directly input into the lifetime fit on an event-by-event basis. A scale factor of 1.22 on this error has been determined to account for its overestimation or underestimation in data (see section 5.2.2). To estimate any systematic bias, caused for instance by selection cuts differing between this analysis and the study in which the error scale factor was determined [59], the scale factor is varied by \pm 0.2 and the fit to data is repeated with the new scale factor. Lowering the scale factor causes a shift of $+3 \mu \mathrm{m}$, enlarging the scale factor causes a shift of $-4 \mu \mathrm{m}$. A systematic error of ${ }_{-4}^{+3} \mu \mathrm{m}$ is assigned.

\section{Background Fraction}

The background fraction $f_{b}$ is a fixed parameter in the lifetime fit which is obtained beforehand from the five parameter fit of a Gaussian and a first order polynomial to the $p K \pi$ invariant mass distribution. It is defined as the number of background events $N_{b}$ present in the signal region of the $p K \pi$ invariant mass distribution divided by the total number of events $N_{s}$ in the signal region. The systematic error associated 
with the uncertainty on the background fraction is estimated by repeating the fit to data with the background fraction varied by $\pm 1 \sigma=0.038$. Lowering the background fraction and repeating the fit yields a five micron shorter result, enlarging the fraction results in a five micron larger result. A systematic error of $\pm 5 \mu \mathrm{m}$ is assigned.

\section{Background from Charm Decays}

The various charm particles produced in semileptonic B decays that could mimic a $\Lambda_{c}$ signal have been discussed in section 4.5.2. While the B decays yielding a $D^{*}$, $D^{0}$ or $D_{s}$ can be efficiently anti-selected by cuts on the respective invariant masses, backgrounds containing a $D^{+}$from $\bar{B}^{0}$ decays pose a problem. Anti-selecting these mesons would at the same time significantly reduce the number of signal events observed. If those background events are unevenly distributed between the signal region and the sidebands in the $p K \pi$ invariant mass plot, the lifetime fit can yield inaccurate results. The realistic Monte Carlo generated to simulate those $D^{+}$events from $\bar{B}^{0}$ decays suggests a surplus of $D^{+}$events in the signal region (see figure 4.26 (b)). The ratio of $D^{+}$events in the signal region to $D^{+}$events in the background region extracted from this $\mathrm{MC}$ is 1.5. Trying to extract this ratio from data is difficult due to the large amount of background events present under the $D^{+}$peak (see figure $4.26(\mathrm{a})$ ).

The fit to the realistic simulation is now repeated using all available $\Lambda_{c} \mathrm{MC}$ events that survive the selection cuts while mixing in events from the $D^{+} \mathrm{MC}$. The amount of $D^{+}$events mixed into the $\Lambda_{c}$ signal MC is calculated from the ratio of $D^{+}$ events in the signal and sideband region, as determined from the $D^{+} \mathrm{MC}$, from the number of $D^{+}$events seen in data and from the number of $\Lambda_{c}$ candidates present in the $3 \sigma$ wide signal peak in the data. The fit yields a $5 \mu \mathrm{m}$ larger result and the final result is thus corrected by $-5 \mu \mathrm{m}$. However, using a smaller sample of $\Lambda_{c}$ $\mathrm{MC}$ events, whose size corresponds to the number of signal events seen in data, and mixing in subsets of the $D^{+} \mathrm{MC}$ sample, the fit result varies by $8 \mu \mathrm{m}$ as compared to the $5 \mu \mathrm{m}$ so that a systematic error of $\pm 8 \mu \mathrm{m}$ is assigned.

Table 6.2 summarises the above results that lead to a total systematic uncertainty on the lifetime measurement of $\pm 21 \mu \mathrm{m}$. 


\begin{tabular}{|c|c|}
\hline Source of Uncertainty & $\boldsymbol{\Delta} \boldsymbol{c} \boldsymbol{(}\left(\boldsymbol{\Lambda}_{\boldsymbol{b}}\right)[\boldsymbol{\mu} \mathbf{m}]$ \\
\hline SVT tracking efficiency & ${ }_{-3}^{+4}$ \\
\hline Resolution of d0 & ${ }_{-9}^{+7}$ \\
\hline Shift of d0 & \pm 15 \\
\hline Input Lifetime & \pm 2 \\
\hline Selection Cuts & +5 \\
\hline Fragmentation & $\approx 0$ \\
\hline$\Lambda_{b}$ polarisation & \pm 3 \\
\hline Error scale factor & ${ }_{-4}^{+3}$ \\
\hline Background fraction & \pm 5 \\
\hline Charm Decays & \pm 8 \\
\hline \hline Sum in quadrature & \pm 21 \\
\hline
\end{tabular}

Table 6.2: Summary table of the sources of the systematic error of the lifetime fit. 


\section{Chapter 7}

\section{Results and Conclusions}

This chapter presents the result of the lifetime fit to events from $186 \mathrm{pb}^{-1}$ of data recorded with the $\mu+\mathrm{SVT}$ trigger at CDF. To begin with all the fixed values, parameters and distributions that are required as input for the lifetime fit are summarised. The free parameters of the fit, determined during the minimisation procedure, are then reviewed. Finally the result of the application of the lifetime fit to the data sample is presented.

\subsection{Fixed Input Values and Distributions}

All parameters and distributions that are required for the lifetime fit were introduced in detail in chapter 5. Here they are briefly summarised and the values the parameters take for the fit to data are quoted.

\section{The Error Scale Factor}

The error scale factor $s$ accounts for overestimation or underestimation of the measurement errors on the pseudo-proper decay length. The error scale factor for data is determined to be 1.22 from a sample that was recorded without the application of the impact parameter cut to allow for prompt events $[57,59]$.

\section{K-factor and $c \tau$ Efficiency}

The K-factor accounts for the missing transverse momentum of the neutrino. In the lifetime fit, the function describing the exponential decay must be convoluted 


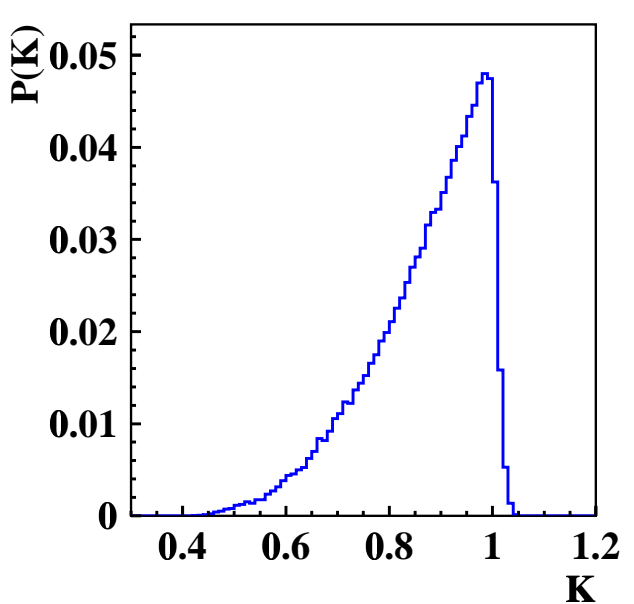

(a)

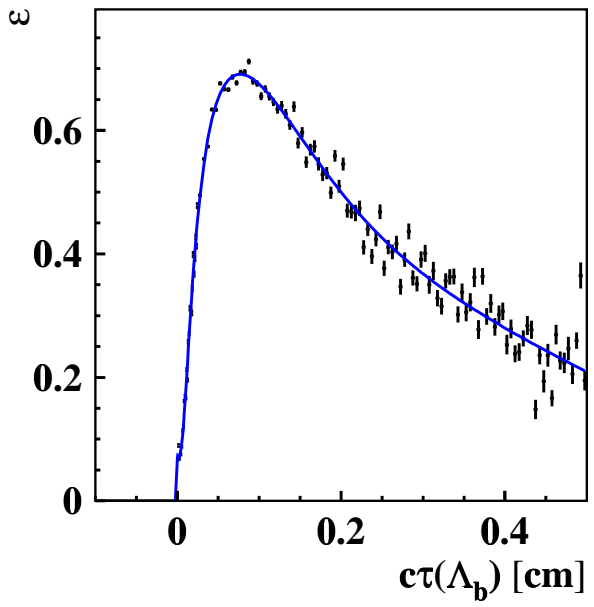

(b)

Figure 7.1: K-factor and $c \tau$ efficiency curve for the lifetime fit to data; the selection cuts applied are summarised in section 4.6.

with the normalised K-factor distribution. This distribution is extracted from the parametric simulation and presented in figure 7.1 (a).

The $c \tau$ efficiency curve accounts for the bias on the $c \tau$ distribution which is introduced via the trigger cut on the impact parameter. This curve too is extracted from the parametric simulation and presented in figure 7.1 (b). The parametrisation of this curve was given in equation 5.2 as

$$
\varepsilon(x)=A \cdot(x+p) \cdot\left(e^{-\lambda_{1} x}+e^{-\lambda_{2} x}+r\right)+t+u \cdot e^{-\frac{(x-v)^{2}}{2 w^{2}}}
$$

and the parameters extracted from the fit are given in table 5.2.

\section{Background Fraction}

The background fraction denotes the amount of background that is contained in the signal region. The signal and background region are the yellow/light-grey and green/dark-grey bands in the $p K \pi$ invariant mass plot which is repeated for conve-

nience in figure 7.2. The background fraction is determined from a five parameter $\chi^{2}$ fit of a Gaussian and a first order polynomial to the $p K \pi$ invariant mass distribution:

$$
p(x)=p 1 \cdot \exp \left\{-0.5 \cdot[(x-p 2) / p 3]^{2}\right\}+p 4+p 5 \cdot x .
$$




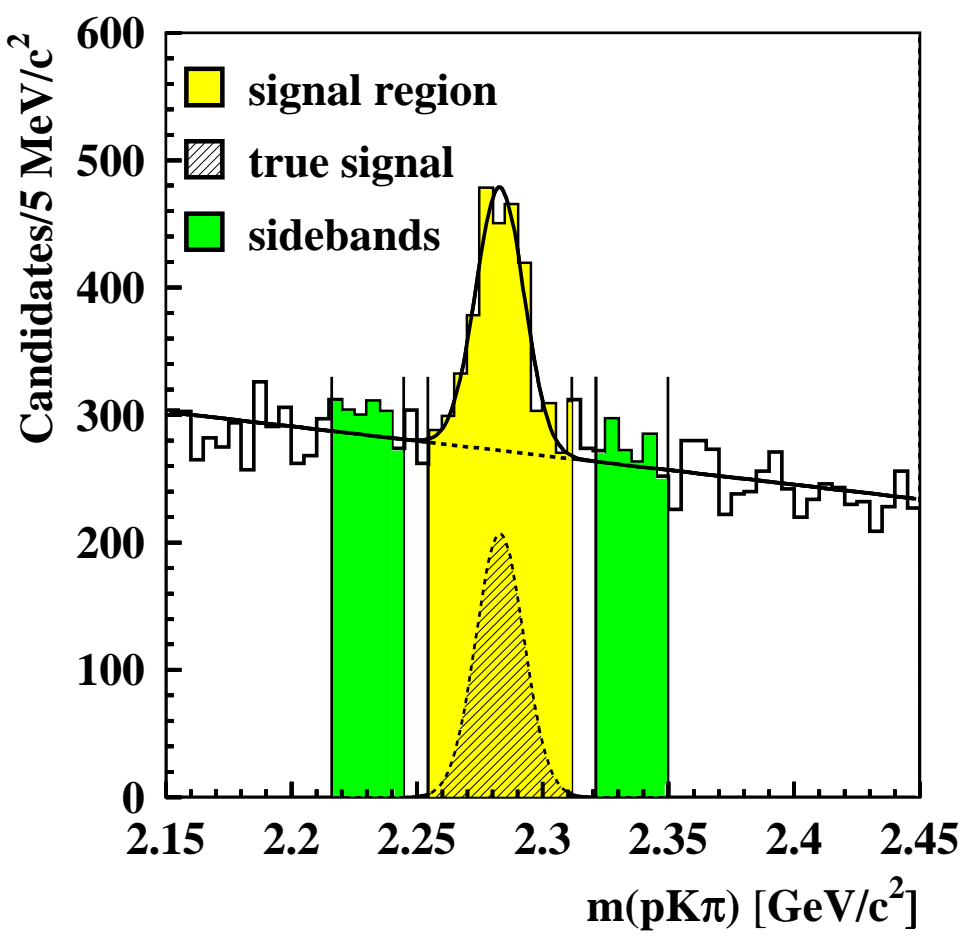

Figure 7.2: Fit of a Gaussian and a first order polynomial to the right-sign $p K \pi$ invariant mass distribution. The signal region and the sidebands are indicated by the yellow (light-grey) and green (dark-grey) bands respectively. The hashed histogram indicates the estimated contribution of true signal events.

The mean value of the Gaussian, $p 2$, corresponds to the mass of the $\Lambda_{c}$ and $p 3$ is the width of the Gaussian. The parameters obtained from this fit are $p 1=206.8 \pm 15.7$, $p 2=2.2829 \pm 0.0008 \mathrm{GeV} / \mathrm{c}^{2}, p 3=0.0096 \pm 0.0007 \mathrm{GeV} / \mathrm{c}^{2}, p 4=794.1 \pm 30.5$ and $p 5=-228.6 \pm 13.2\left(\mathrm{GeV} / \mathrm{c}^{2}\right)^{-1}$.

The number of events in the signal and background region are calculated from these parameters as follows:

$$
N_{b}=\frac{6 p 3(p 5 p 2+p 4)}{\text { binwidth }}
$$

and

$$
N_{s}=N_{b}+0.99 \frac{\sqrt{2 \pi} p 1 p 3}{\text { binwidth }} .
$$

The factor 0.99 accounts for the fact that the $3 \sigma$-wide bands contain approximately $99 \%$ of the events in the signal peak. With a binwidth of 0.005 this results in a background fraction $f_{b}=\frac{N_{b}}{N_{s}}$ of 0.761 and an estimated number of true signal events 
in the signal region (indicated by the hashed Gaussian in figure 7.2) of 981, which corresponds to $99 \%$ of the 991 events overall contained in the Gaussian. Looking at figure 7.2 it can be noted that the number of events in the $3 \sigma$ wide sidebands exceeds the number of events as estimated by the polynomial fit. To account for a possible upward or downward fluctuation of events in the signal region, the background fraction is varied in the lifetime fit by \pm 0.037 (see chapter 6). This variation can be interpreted as an upward or downward fluctuation of the number of background events of approximately 150 and causes a shift of $5 \mu \mathrm{m}$ in the fit result.

\section{$c \tau^{\prime}\left(\Lambda_{b}\right)$ and $\sigma\left(c \tau^{\prime}\right)$}

The final event-by-event inputs to the lifetime fit are the values of the pseudo-proper decay length of the $\Lambda_{b}, c \tau^{\prime}\left(\Lambda_{b}\right)$, and the error on this quantity, $\sigma\left(c \tau^{\prime}\right)$, for all events in the signal $\left(N_{s}\right)$ and the sideband $\left(N_{b}\right)$ region. The borders of the signal and sideband region are also defined by the results from the five parameter fit to the $p K \pi$ invariant mass plot. Using $p 2=\mu$ and $p 3=\sigma$, the signal region is defined by $\mu-3 \sigma \leq \mathrm{m}(p K \pi) \leq \mu+3 \sigma$ and the sidebands are defined by $\mu-7 \sigma \leq \mathrm{m}(p K \pi)$ $\leq \mu-4 \sigma$ and $\mu+4 \sigma \leq \mathrm{m}(p K \pi) \leq \mu+7 \sigma$.

\subsection{Free Parameters of the Lifetime Fit}

As described in chapter 5, the fit is a minimisation of the following function:

$$
-2 \ln \mathcal{L}=-2\left(\sum_{i=1}^{N_{s}} \ln \mathcal{P}+\sum_{i=1}^{N_{b}} \ln \mathcal{P}_{b}\right),
$$

where $\mathcal{P}$ and $\mathcal{P}_{b}$ are the normalised versions of the functions $\mathcal{F}$ and $\mathcal{F}_{b}$. $\mathcal{F}$ itself is composed of a term describing pure signal events and a term describing background events only:

$$
\mathcal{F}=\left(1-f_{b}\right) \mathcal{F}_{s}+f_{b} \mathcal{F}_{b} .
$$

The only variable parameter of the fit that originates from the signal function $\mathcal{F}_{s}$ is the lifetime $c \tau$ of the $\Lambda_{b}$ baryon which is sought in this thesis. The background function on the other hand contributes six free parameters. It is comprised of a prompt component and a single exponentially decaying component, both of which 
are convoluted with a Gaussian resolution function. After normalisation the term describing background events becomes:

$$
\mathcal{P}_{b}=(1-f r a c 1) \cdot f_{\delta}+(f r a c 1) \cdot f_{\exp }
$$

with

$$
f_{\delta}=\frac{1}{\sqrt{2 \pi} \sigma_{0}} \cdot \exp \left[-0.5\left(\frac{x-X_{0}}{\sigma_{0}}\right)^{2}\right]
$$

and

$$
f_{\exp }=\frac{1}{c \tau_{b}} \cdot \exp \left[\frac{1}{2}\left(\frac{\sigma_{b}}{c \tau_{b}}\right)^{2}-\frac{x-X_{b}}{c \tau_{b}}\right] \cdot\left[1-\operatorname{freq}\left(\frac{\sigma_{b}}{c \tau_{b}}-\frac{x-X_{b}}{\sigma_{b}}\right)\right]
$$

\subsection{The Lifetime Fit}

The values for the variables $c \tau^{\prime}\left(\Lambda_{b}\right)$ and $\sigma\left(c \tau^{\prime}\right)$ are recorded for every event in the signal and the sideband region. Using the MINUIT package [60] the minimisation is then carried out to determine the $i=$ seven free parameters $\alpha_{i}$ of the fit: $\boldsymbol{\alpha}=\left(c \tau, f r a c 1, \sigma_{0}, X_{0}, c \tau_{b}, X_{b}, \sigma_{b}\right)$. The fit converged and figure 7.3 presents its results. Figure 7.3 (a) shows the histogram of events from the signal region (points with error bars) together with the fitted function (smooth curve) and figure 7.3 (b) shows the fit for events in the sideband region. The value of the decay length of the $\Lambda_{b}$ determined by the described fit is

$$
\mathrm{c} \tau=387 \pm 33 \text { (stat.) } \mu \mathrm{m} \text {. }
$$

The results for all seven fit parameters are detailed in table 7.1 and figure 7.3 (c) shows the fitted curve of figure 7.3 (a) together with its components, the signal events, the prompt background and the exponentially decaying background. Together with the systematic uncertainty on the fit determined in chapter 6 the final result of the lifetime fit is:

$$
\mathrm{c} \tau=387 \pm 33 \text { (stat.) } \pm 21 \text { (syst.) } \mu \mathrm{m}
$$

equivalent to

$$
\tau=1.29 \pm 0.11 \text { (stat.) } \pm 0.07 \text { (syst.) ps. }
$$




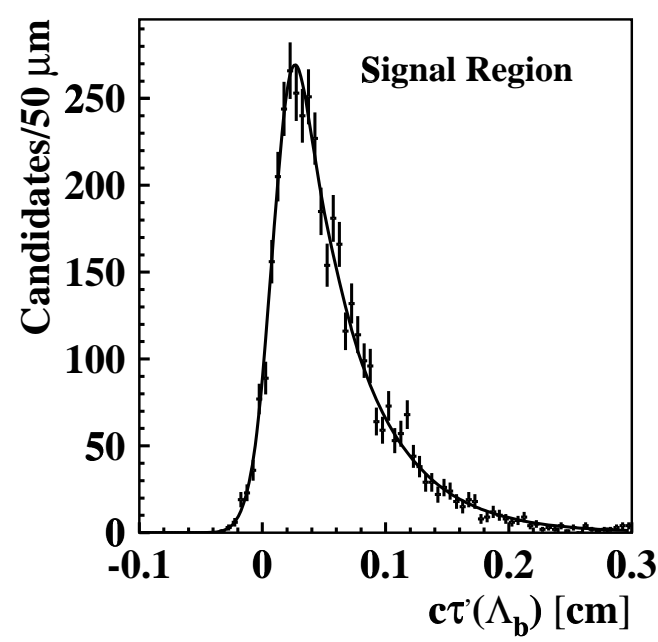

(a)

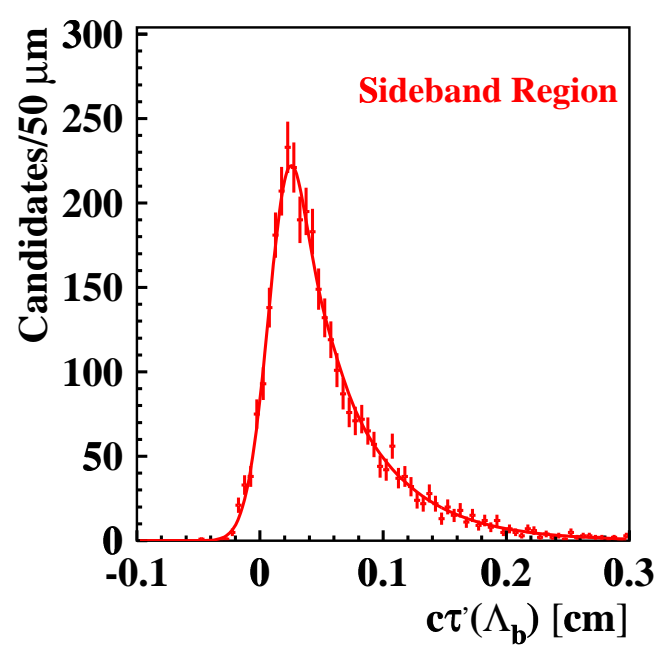

(b)

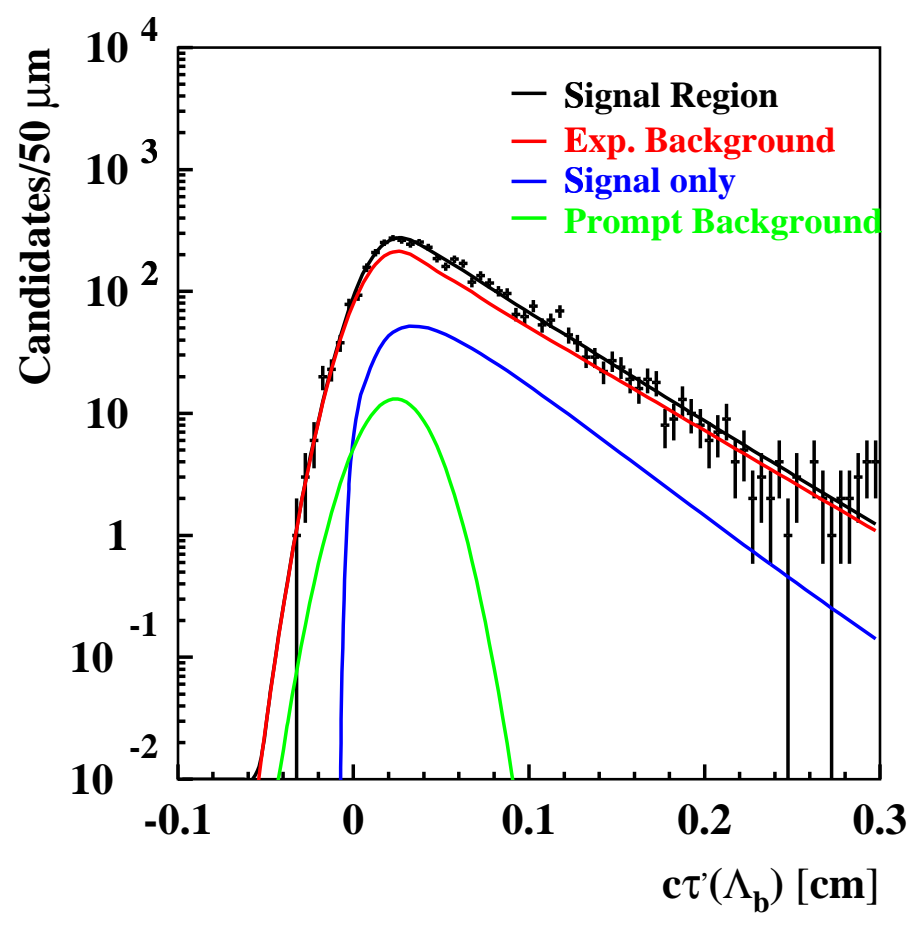

(c)

Figure 7.3: Lifetime fit to the data. Figure (a) shows events from the signal region (data points with error bars) together with the fitted function (smooth curve). Figure (b) shows events from the sideband region. Figure (c) shows the fitted curve from (a) on a logarithmic scale, together with the signal contribution and the contribution from the exponential and the prompt background. 


\begin{tabular}{|c|c|}
\hline Parameter & Fit Result \\
\hline$c \tau\left(\Lambda_{b}\right)[\mathrm{cm}]$ & $0.0387 \pm 0.0033$ \\
\hline$X_{0}[\mathrm{~cm}]$ & $0.024 \pm 0.002$ \\
\hline$\sigma_{0}[\mathrm{~cm}]$ & $0.0176 \pm 0.0007$ \\
\hline$f r a c 1$ & $0.41 \pm 0.04$ \\
\hline$X_{b}[\mathrm{~cm}]$ & $0.047 \pm 0.005$ \\
\hline$\sigma_{b}[\mathrm{~cm}]$ & $0.012 \pm 0.002$ \\
\hline$c \tau_{b}[\mathrm{~cm}]$ & $0.052 \pm 0.001$ \\
\hline
\end{tabular}

Table 7.1: Fit parameters obtained in the final lifetime fit to data. The value for $c \tau\left(\Lambda_{b}\right)$ is corrected for the presence of background from $D^{+}$events.

\subsection{Conclusions}

Using $186 \mathrm{pb}^{-1}$ of data recorded with the $\mu+\mathrm{SVT}$ trigger at CDF, an estimated 991 $\Lambda_{b}$ decays were reconstructed from their semileptonic decay $\Lambda_{b}^{0} \rightarrow \Lambda_{c}^{+} \mu^{-} \bar{\nu}_{\mu}$ with the subsequent decay $\Lambda_{c}^{+} \rightarrow p K^{-} \pi^{+}$.

For the first time in a hadron collider experiment a cut on the impact parameter was applied at the trigger level to enhance the fraction of events from decays of long-lived particles. The bias thus introduced to the decay length distribution of the $\Lambda_{b}$ has been accounted for in the unbinned log likelihood fit to the pseudo-proper decay length of the $\Lambda_{b}$ candidates. The fit yields a mean decay length and a mean lifetime of

$$
\begin{gathered}
\mathrm{c} \tau=387 \pm 33 \text { (stat.) } \pm 21 \text { (syst.) } \mu \mathrm{m} \\
\tau=1.29 \pm 0.11 \text { (stat.) } \pm 0.07 \text { (syst.) ps. }
\end{gathered}
$$

This result agrees with the $1.229 \pm 0.080$ ps quoted by the Particle Data Group [22] and the LEP B Lifetime working group (which quote the same result but with an asymmetric error of ${ }_{-0.079}^{+0.081} \mathrm{ps}$ [67]). Table 7.2 lists the various measurements that were used by the LEP B Lifetime working group to obtain the combined value and figure 7.4 presents these results graphically. The result obtained in this thesis, which is added for comparison to figure 7.4, agrees with the individual results quoted within the respective errors. The statistical error observed shows an improvement in accuracy compared to all of the above measurements. The application of a cut on the track's impact parameter at the secondary trigger level has proven a viable method of selecting events for a lifetime analysis. 


\begin{tabular}{|c|c|c|c|c|}
\hline Experiment & Channel & Data Set & $\boldsymbol{\tau}\left(\Lambda_{b}\right)[\mathbf{p s}]$ & Ref. \\
\hline $\mathrm{ALEPH}$ & $\Lambda_{c} l$ & $91-95$ & $1.18_{-0.12}^{+0.13} \pm 0.03$ & {$[68]$} \\
\hline $\mathrm{ALEPH}$ & $\Lambda l^{-} l^{+}$ & $91-95$ & $1.30_{-0.21}^{+0.26} \pm 0.04$ & {$[68]$} \\
\hline $\mathrm{CDF}$ & $\Lambda_{c} l$ & $91-95$ & $1.32 \pm 0.15 \pm 0.06$ & {$[55]$} \\
\hline $\mathrm{DELPHI}$ & $\Lambda_{c} l$ & $91-94$ & $1.11_{-0.18}^{+0.19} \pm 0.05$ & {$[69]$} \\
\hline $\mathrm{OPAL}$ & $\Lambda_{c} l \& \Lambda l^{-} l^{+}$ & $90-95$ & $1.29_{-0.22}^{+0.24} \pm 0.06$ & {$[56]$} \\
\hline
\end{tabular}

Table 7.2: Results of measurements of the $\Lambda_{b}$ lifetime from previous experiments.

One motivation for this lifetime measurement is the desire to provide a measurement of the ratio of lifetimes $\tau\left(\Lambda_{b}\right) / \tau\left(B_{d}\right)$, a ratio which is also accessible to theoretical calculations in heavy quark physics. As such this is a very interesting measurement for theorists. Using the world average of the $B_{d}$ lifetime quoted by the Particle Data Group [1] as $1.548 \pm 0.032$ ps together with the $\Lambda_{b}$ lifetime measured in this thesis, this ratio is

$$
\frac{\tau\left(\Lambda_{b}\right)}{\tau\left(B_{d}\right)}=0.833 \pm 0.086
$$

After the inclusion of various non-spectator diagrams (see section 2.5) the theoretical prediction for this lifetime ratio is $0.90 \pm 0.05[21,70]$. Although measurement and theoretical prediction agree within their uncertainties, the measurement is still statistically limited, so that this area remains an exciting field of study for the future. During the time of writing, CDF continues to take data and furthermore to update and perfect the description of the detector components. While the larger amount of data will reduce the statistical error, an updated detector description will ultimately yield a smaller systematic error and allow for a more aggressive candidate selection. A better signal-to-background ratio would then be possible by including more stringent requirements in the particle identification. By accumulating more data, CDF will thus ultimately provide the most accurate measurement of the $\Lambda_{b}$ lifetime until the LHC is well underway. 


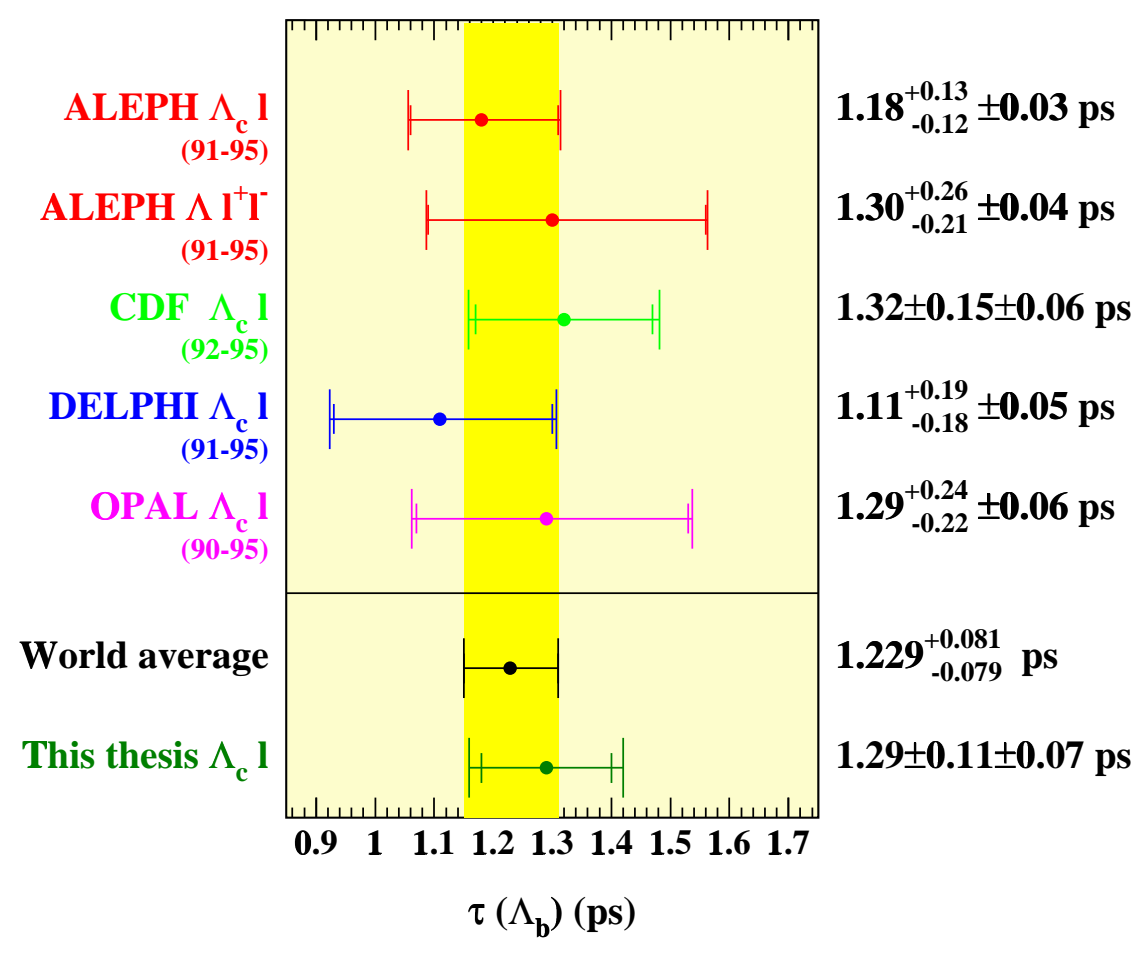

Figure 7.4: Results of measurements of the $\Lambda_{b}$ lifetime from previous experiments [67]. The result obtained in this thesis is added for comparison to the standard plot from the LEP B lifetimes working group [67]. 


\section{Appendix A}

\section{Data - Monte Carlo Comparison}

This appendix shows a direct comparison of data and MC distributions as well as a comparison of the realistic simulation and the parametric simulation. The distributions presented are mainly of those variables considered as cut variables in section 4.4.3 supplemented by a few additional distributions such as $\mathrm{p}_{\mathrm{T}} \mathrm{l}, \eta$ and $\mathrm{d} 0$. Figures A.1-A.4 show a comparison between the data and the realistic simulation. The realistic simulation contains only signal events. The data distributions are obtained using the invariant mass of the $\Lambda_{c}$ candidates for sideband subtraction. The distributions show reasonable agreement within the uncertainty of the data distributions. The sample of the realistic simulation is used to optimise the cut values for this analysis as well as to test the lifetime fit and aid in the determination of its systematic uncertainties.

Figures A.5-A.8 show a comparison between data and the parametric simulation. The distributions of the fit probability as well as the error on the quantities $L_{x y}\left(\Lambda_{b}\right)$ and $L_{x y}\left(\Lambda_{c}\right)$ are here omitted as they are not calculated in the parametric simulation. It should be noted that although the general agreement between data and $\mathrm{MC}$ is again good, there is a discrepancy in the variable $c \tau\left(\Lambda_{c}\right)$. The origin of this discrepancy are resolution effects in conjunction with the short lifetime of the $\Lambda_{c}$. The $c \tau\left(\Lambda_{c}\right)$ plot in figure A.8 shows however that the cut values are chosen such that almost all signal events are accepted. As such the discrepancy between data and MC for this distribution is negligible for this analysis.

Figures A.9-A.12 finally compare the realistic simulation and the parametric simulation. Looking at the distribution of $c \tau\left(\Lambda_{c}\right)$ shows again the discrepancy mentioned above and the figure indicates that the cuts chosen are safe. 

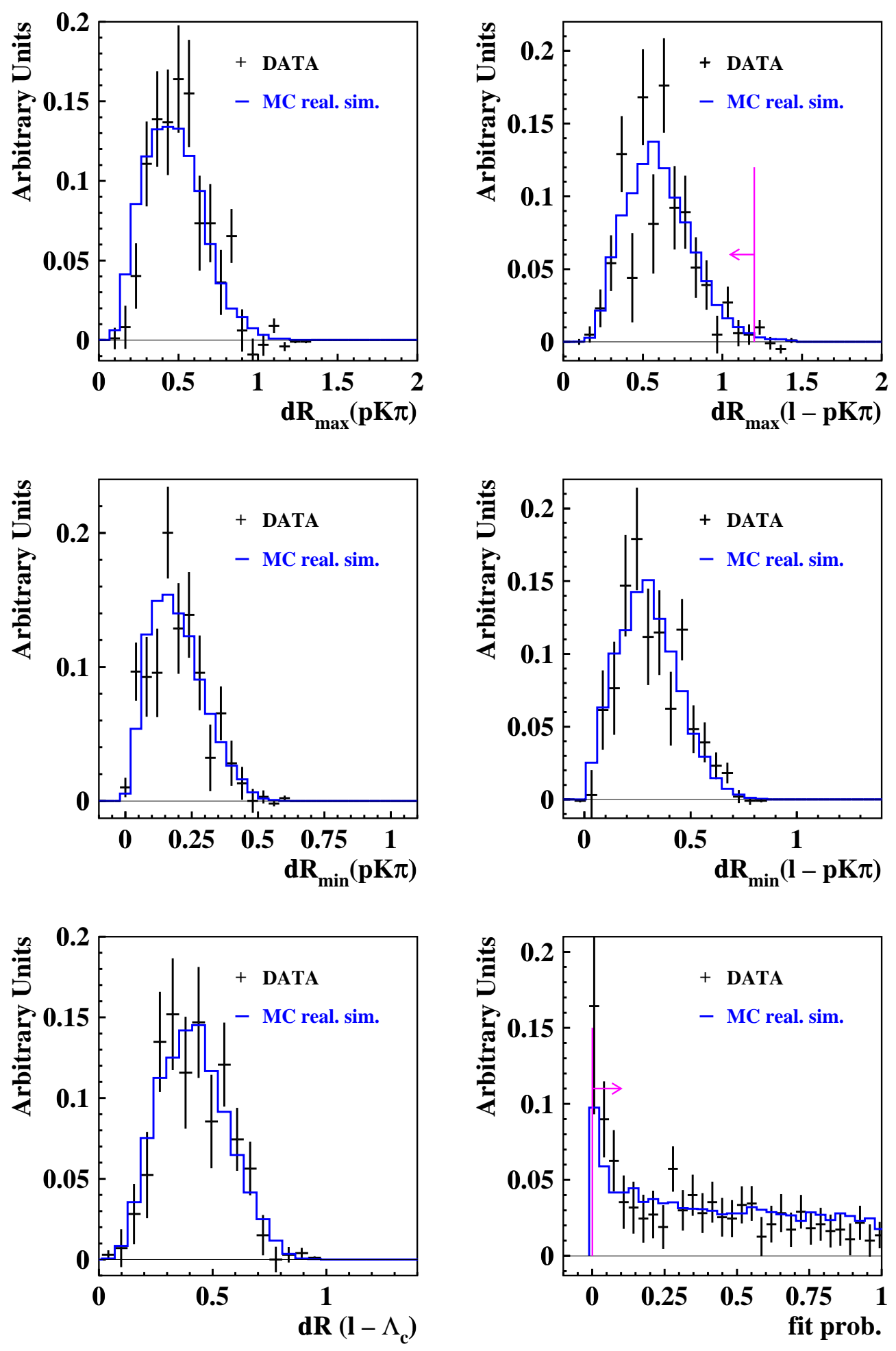

Figure A.1: Comparison between signal distributions of the realistic simulation and data (sideband subtracted). All cuts used to select signal candidates are applied apart from the cut on the variable itself. The cut value, if a cut is applied for the respective variable, is indicated by the vertical line with an arrow pointing towards the accepted region. 

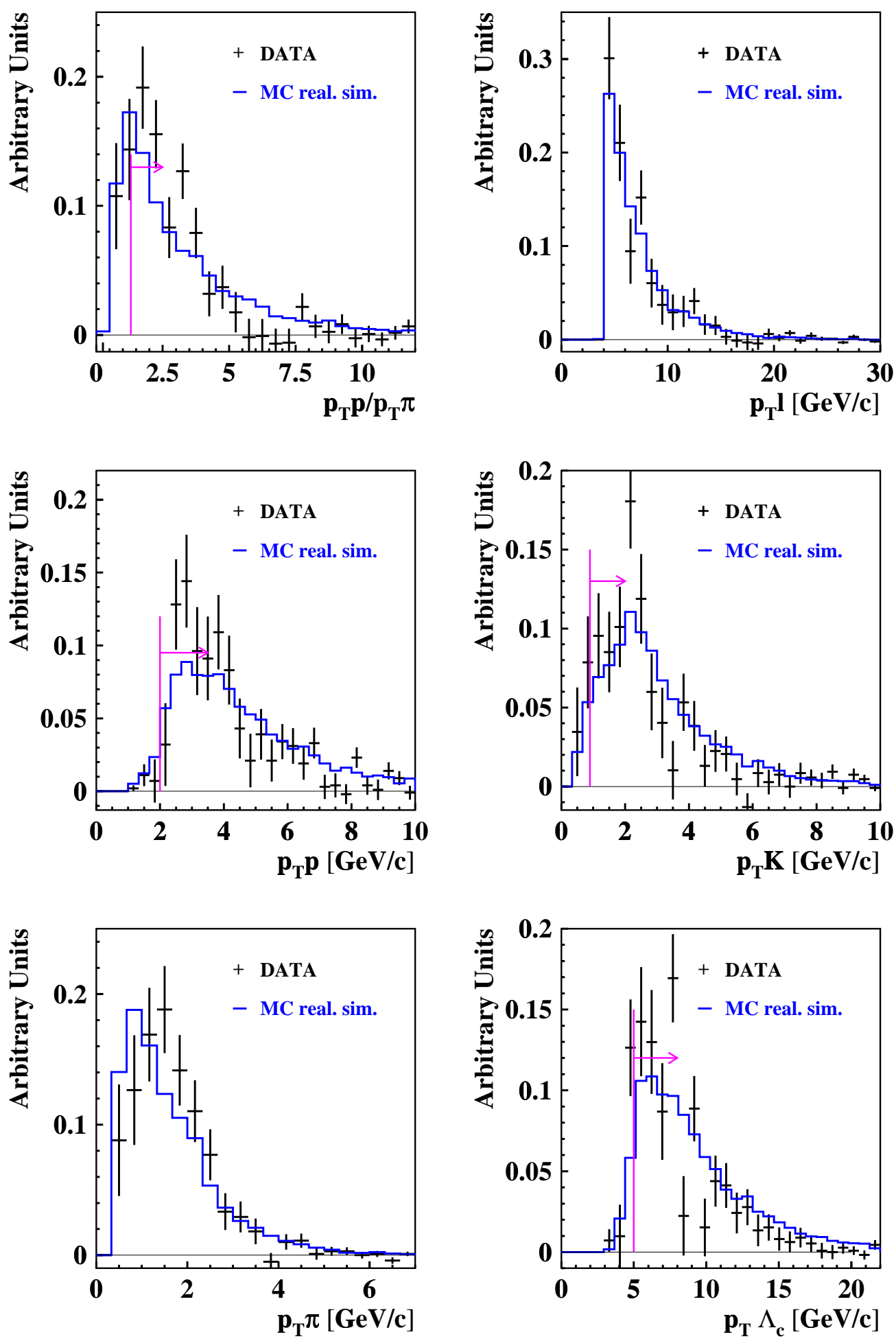

Figure A.2: Comparison between signal distributions of the realistic simulation and data (sideband subtracted). All cuts used to select signal candidates are applied apart from the cut on the variable itself. The cut value, if a cut is applied for the respective variable, is indicated by the vertical line with an arrow pointing towards the accepted region. 

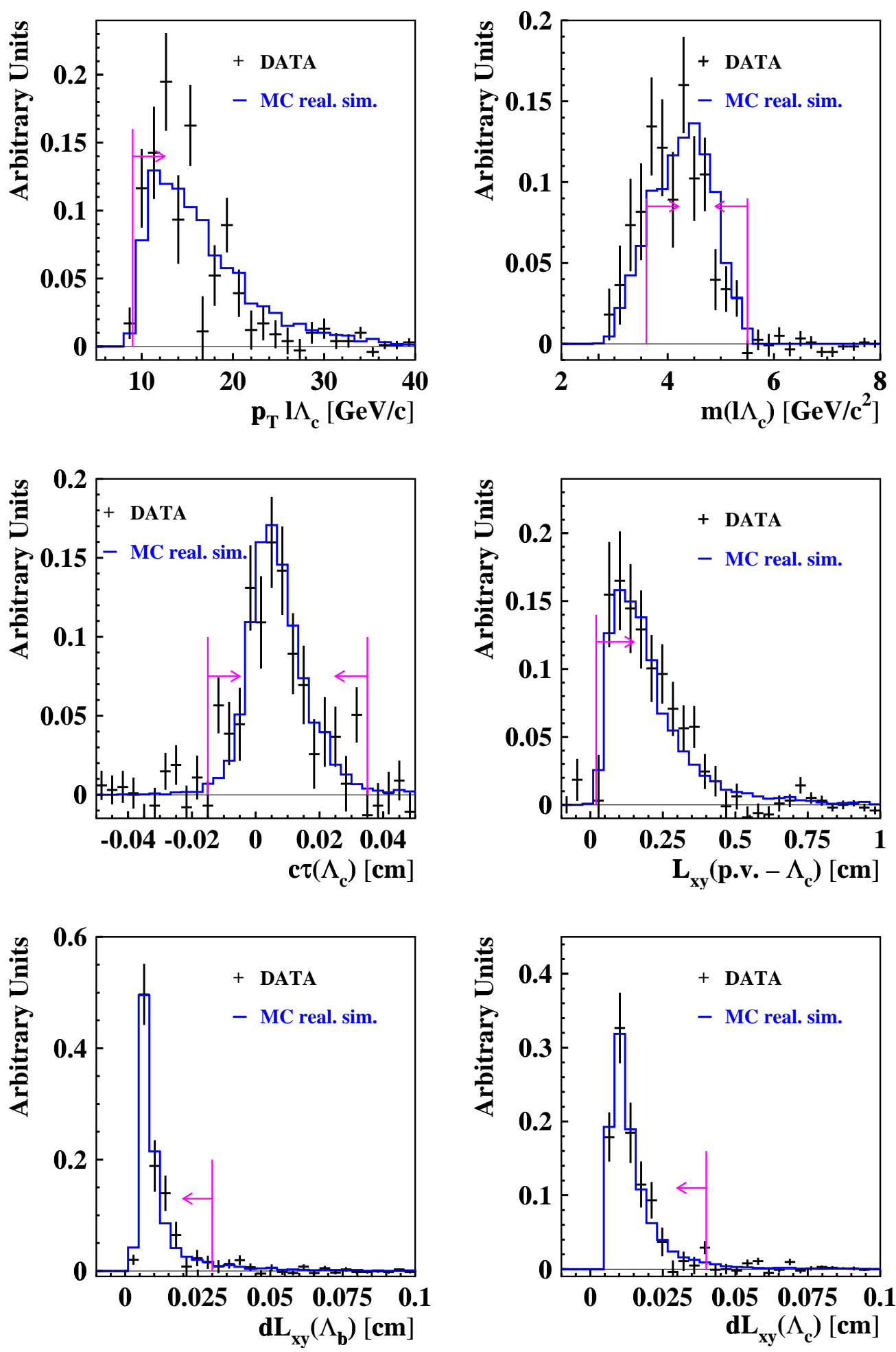

Figure A.3: Comparison between signal distributions of the realistic simulation and data (sideband subtracted). All cuts used to select signal candidates are applied apart from the cut on the variable itself. The cut value, if a cut is applied for the respective variable, is indicated by the vertical line with an arrow pointing towards the accepted region. 

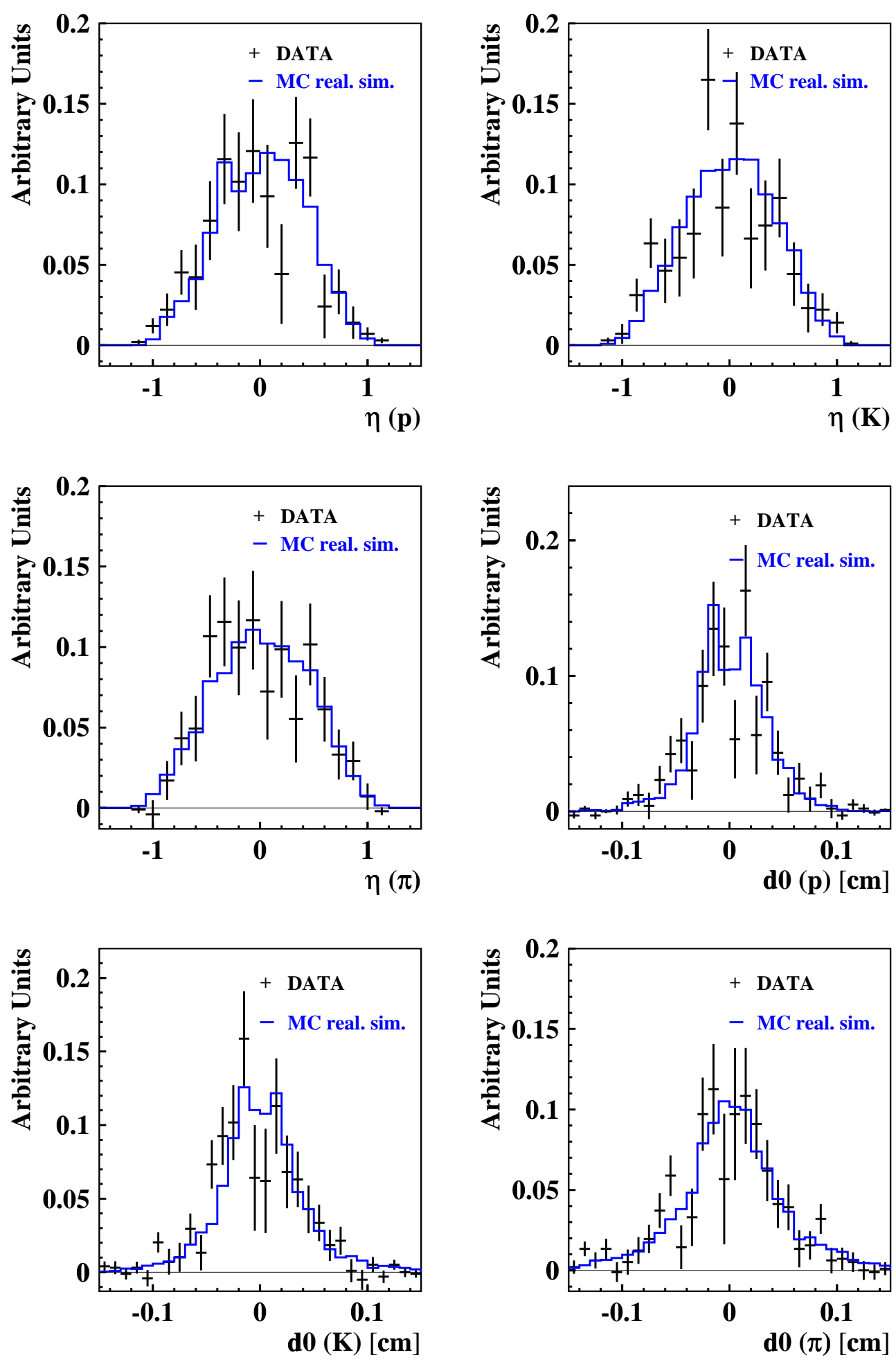

Figure A.4: Comparison between signal distributions of the realistic simulation and data (sideband subtracted). All cuts used to select signal candidates are applied apart from the cut on the variable itself. The cut value, if a cut is applied for the respective variable, is indicated by the vertical line with an arrow pointing towards the accepted region. 

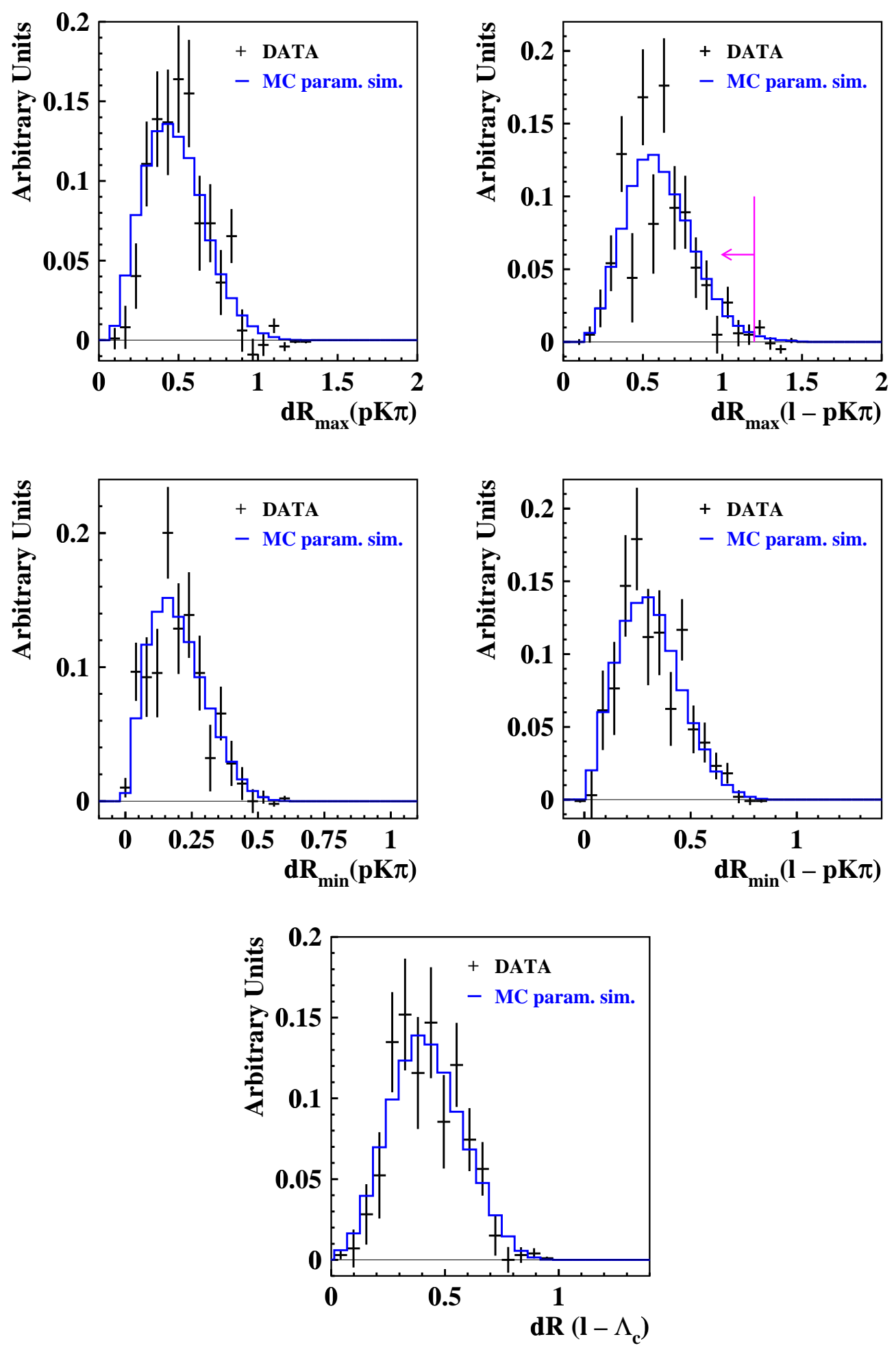

Figure A.5: Comparison between signal distributions of the parametric simulation and data (sideband subtracted). All cuts used to select signal candidates are applied apart from the cut on the variable itself. The cut value, if a cut is applied for the respective variable, is indicated by the vertical line with an arrow pointing towards the accepted region. 

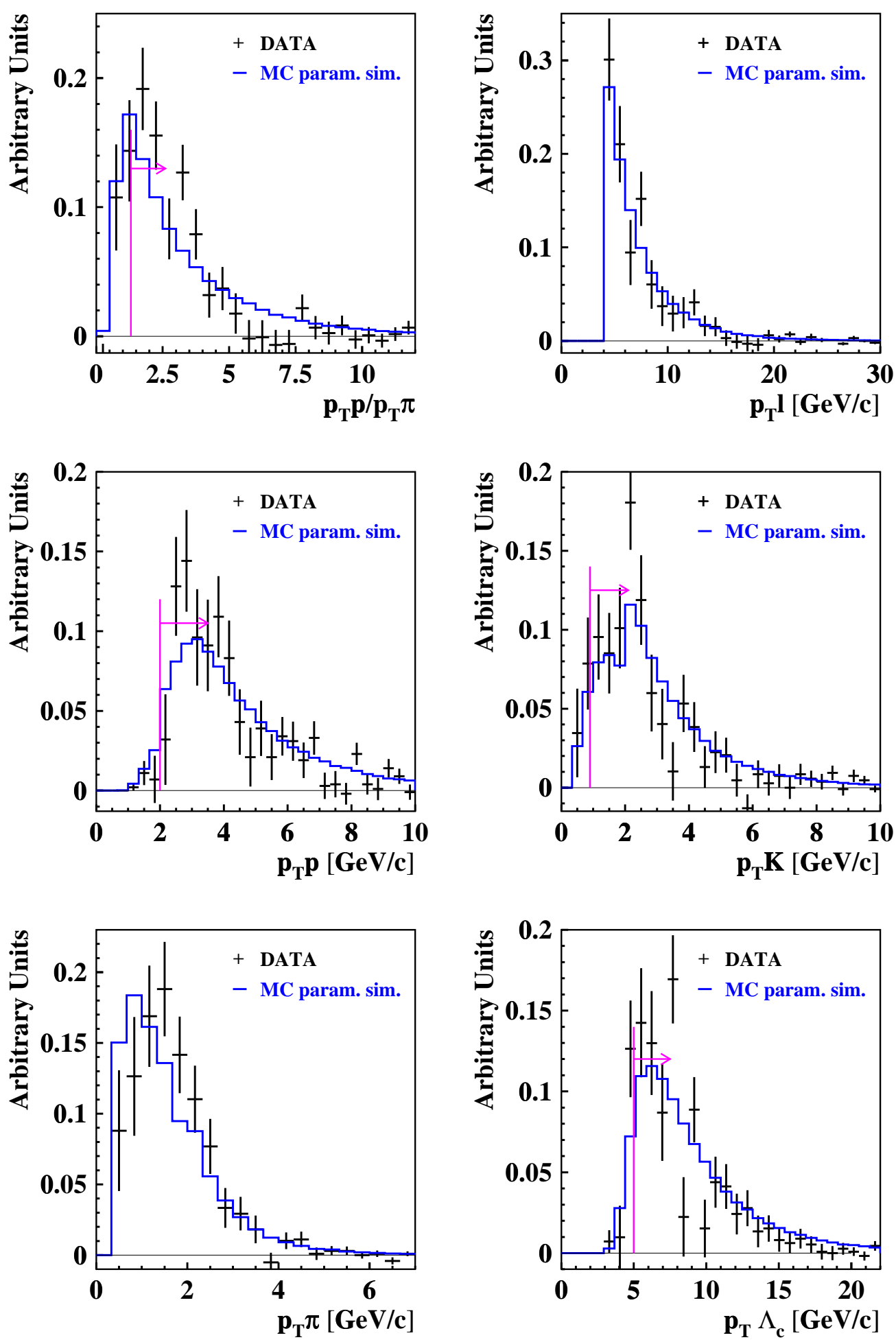

Figure A.6: Comparison between signal distributions of the parametric simulation and data (sideband subtracted). All cuts used to select signal candidates are applied apart from the cut on the variable itself. The cut value, if a cut is applied for the respective variable, is indicated by the vertical line with an arrow pointing towards the accepted region. 

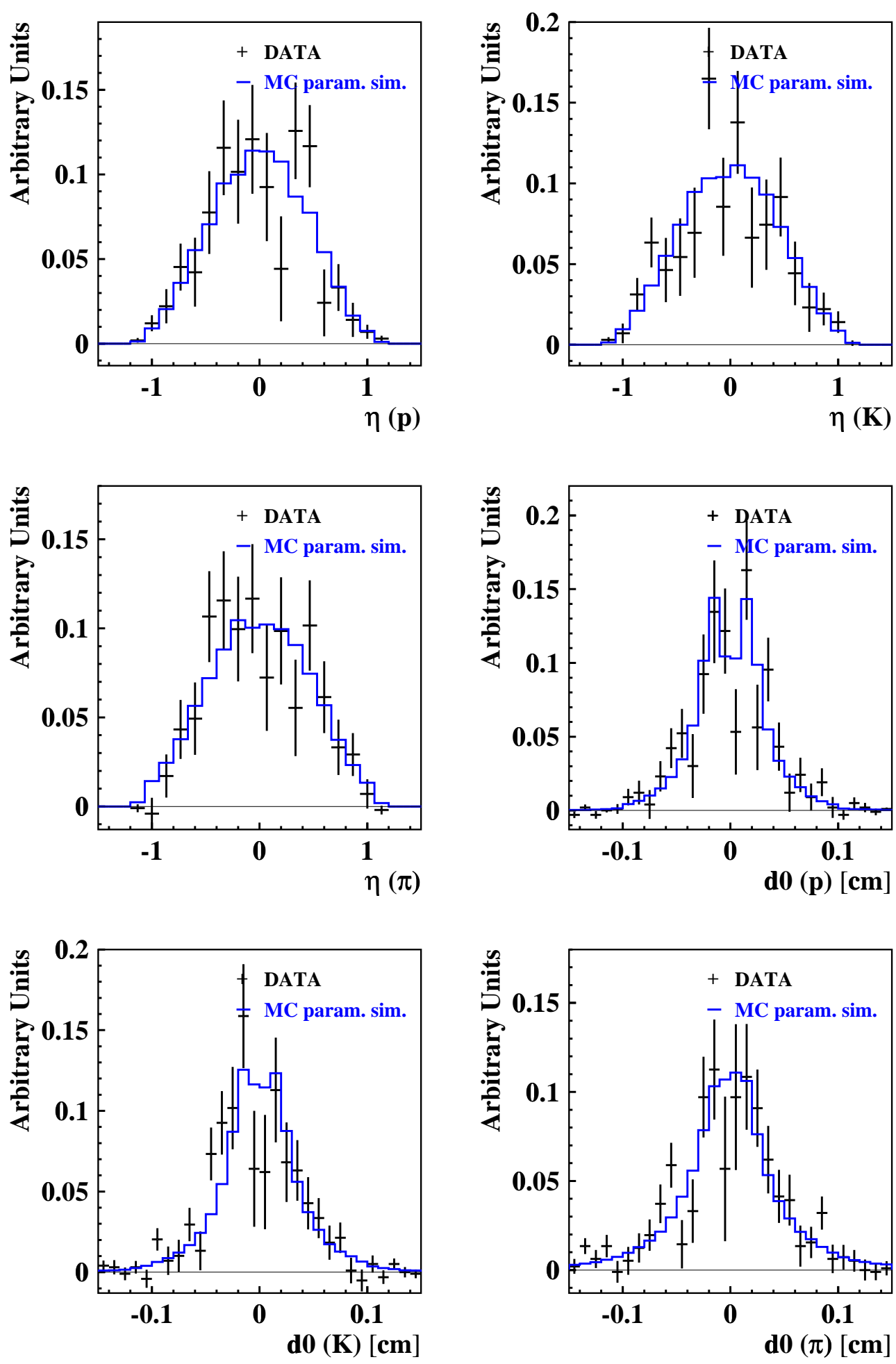

Figure A.7: Comparison between signal distributions of the parametric simulation and data (sideband subtracted). All cuts used to select signal candidates are applied apart from the cut on the variable itself. The cut value, if a cut is applied for the respective variable, is indicated by the vertical line with an arrow pointing towards the accepted region. 

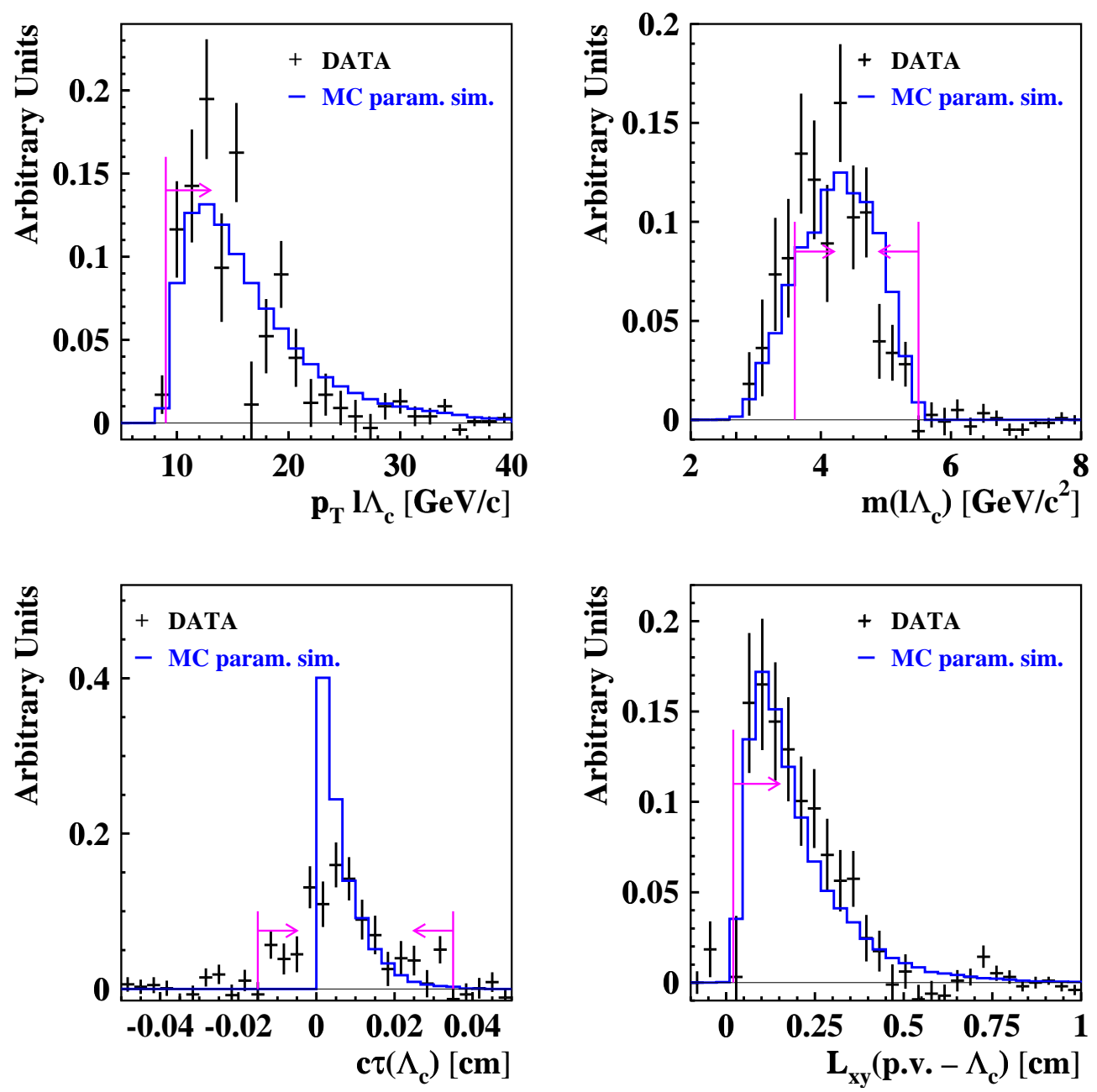

Figure A.8: Comparison between signal distributions of the parametric simulation and data (sideband subtracted). All cuts used to select signal candidates are applied apart from the cut on the variable itself. The cut value, if a cut is applied for the respective variable, is indicated by the vertical line with an arrow pointing towards the accepted region. 

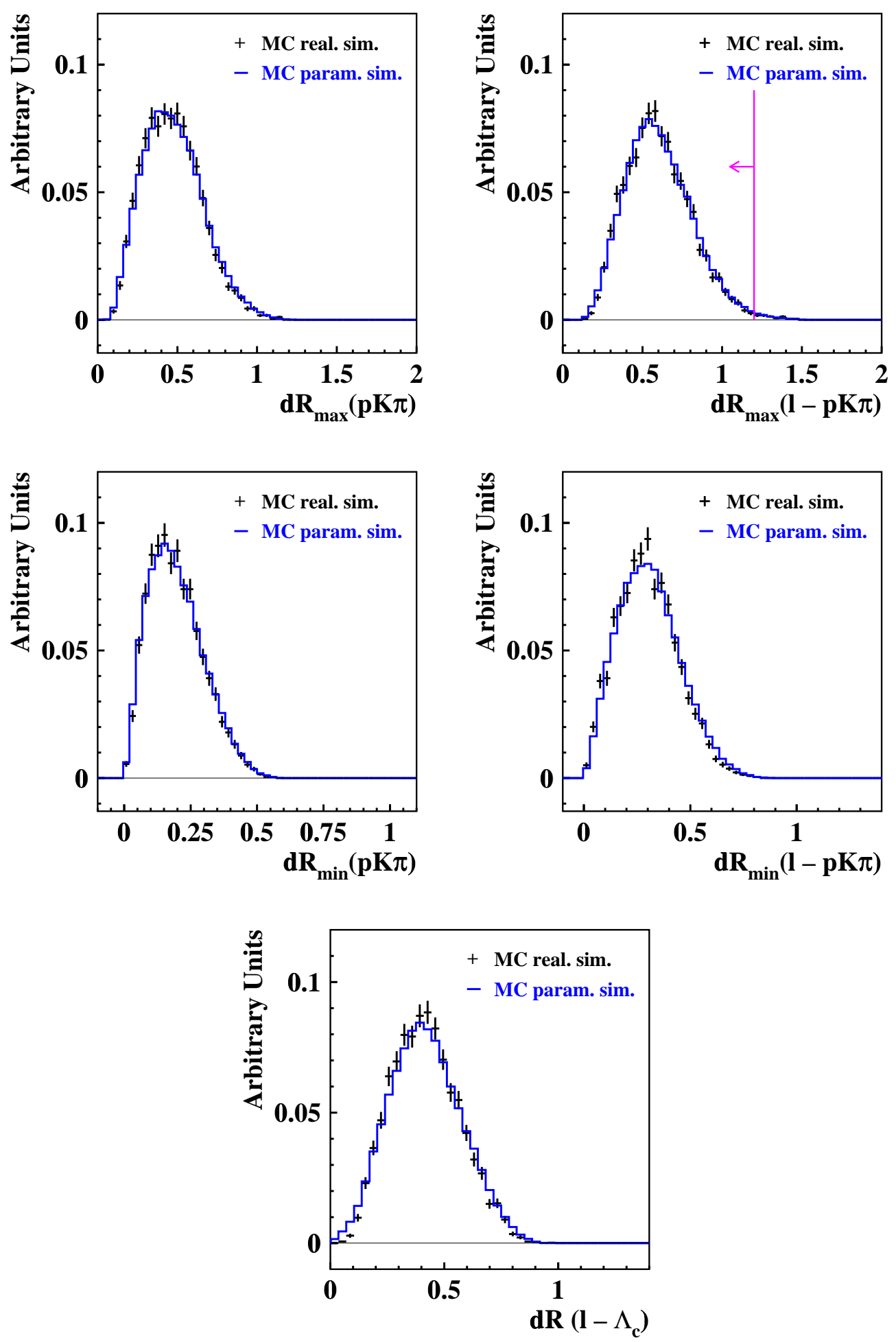

Figure A.9: Comparison between signal distributions of the parametric simulation and the realistic simulation. All cuts used to select signal candidates are applied apart from the cut on the variable itself. The cut value, if a cut is applied for the respective variable, is indicated by the vertical line with an arrow pointing towards the accepted region. 

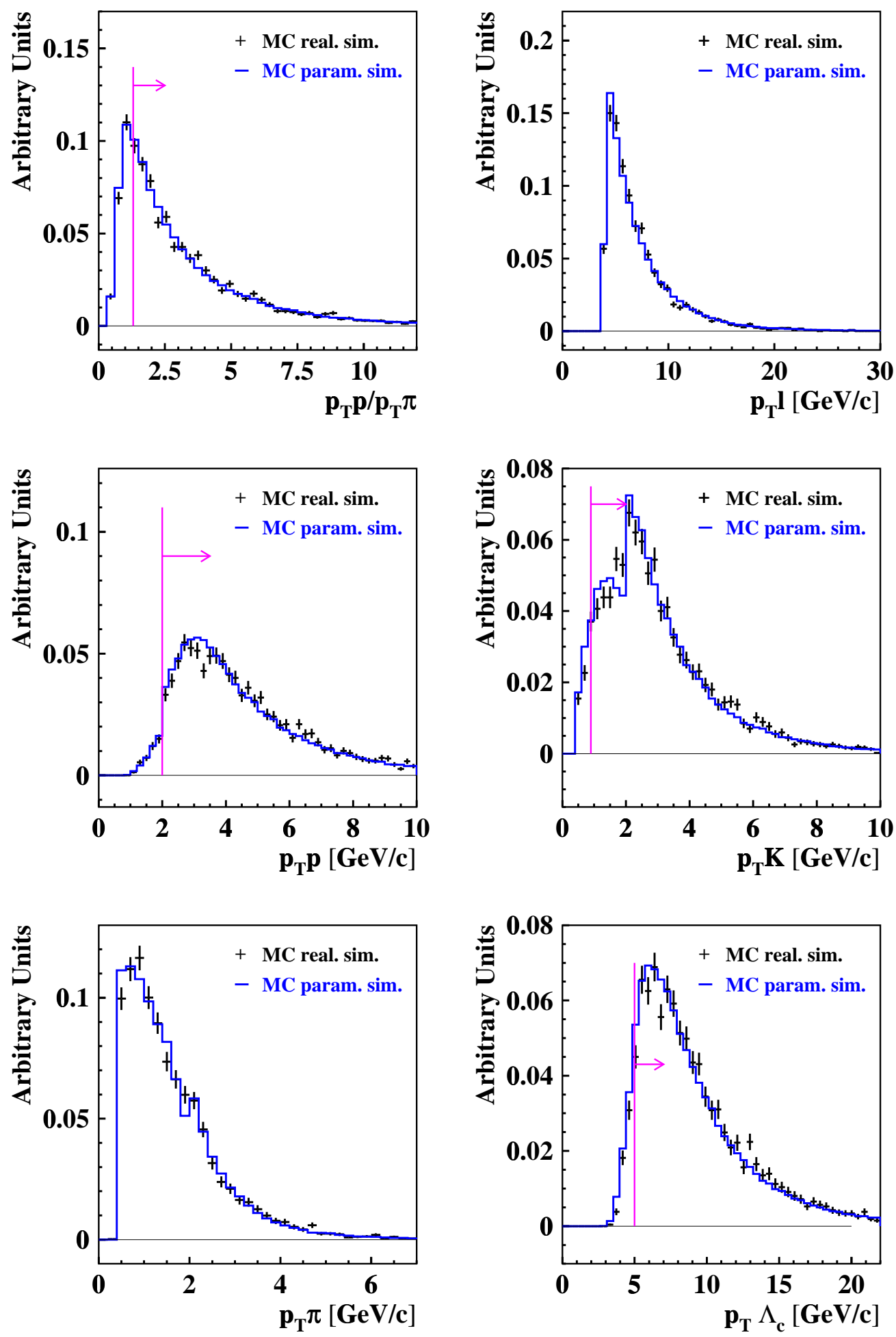

Figure A.10: Comparison between signal distributions of the parametric simulation and the realistic simulation. All cuts used to select signal candidates are applied apart from the cut on the variable itself. The cut value, if a cut is applied for the respective variable, is indicated by the vertical line with an arrow pointing towards the accepted region. 

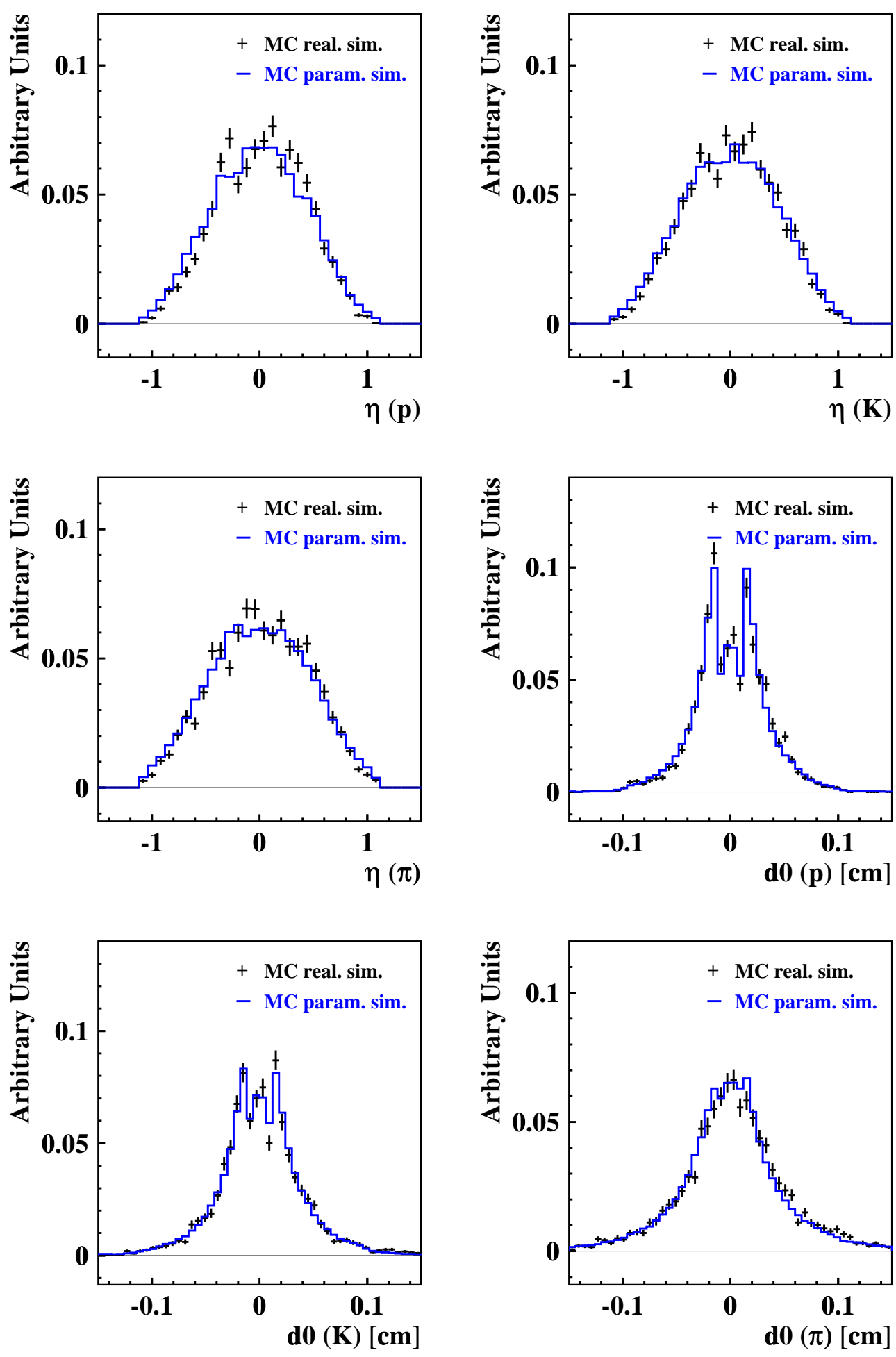

Figure A.11: Comparison between signal distributions of the parametric simulation and the realistic simulation. All cuts used to select signal candidates are applied apart from the cut on the variable itself. The cut value, if a cut is applied for the respective variable, is indicated by the vertical line with an arrow pointing towards the accepted region. 

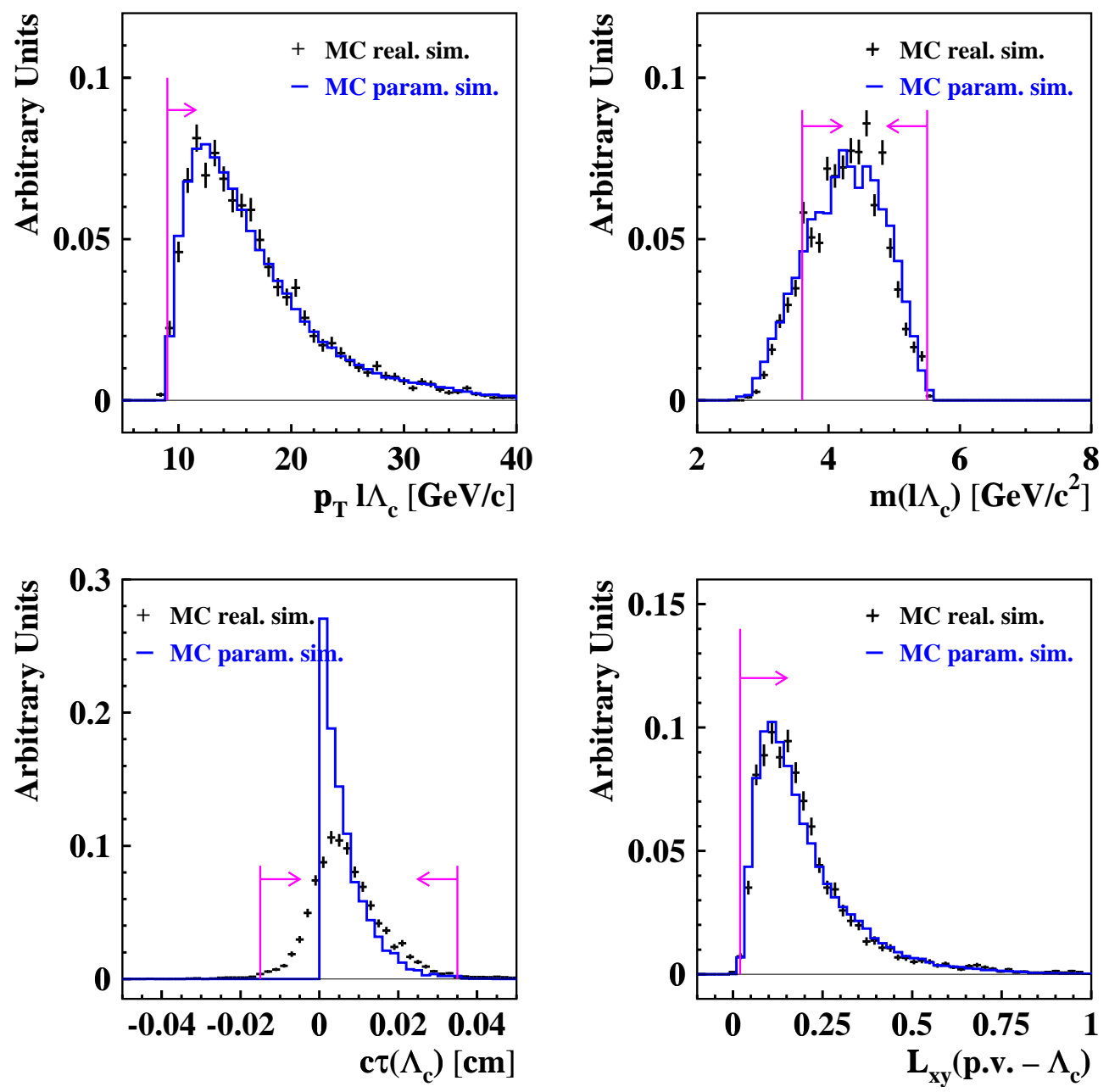

Figure A.12: Comparison between signal distributions of the parametric simulation and the realistic simulation. All cuts used to select signal candidates are applied apart from the cut on the variable itself. The cut value, if a cut is applied for the respective variable, is indicated by the vertical line with an arrow pointing towards the accepted region. 


\section{Appendix B}

\section{Integration of the Fit Functions}

This appendix presents explicit calculations concerning the fit functions introduced in section 5.2.2.

\section{B.1 Calculation of the Signal Function}

Omitting the convolution with the K-factor, the signal function $\mathcal{F}_{s}$ is given by

$$
\mathcal{F}_{s}=\int_{-\infty}^{\infty} d y \theta(x-y) e^{\frac{-K(x-y)}{c \tau}} \cdot \varepsilon(K(x-y)) \cdot \frac{1}{s \sigma \sqrt{2 \pi}} \cdot e^{\frac{-y^{2}}{2(s \sigma)^{2}}},
$$

with

$$
\varepsilon(x)=A \cdot(x+p) \cdot\left(e^{-\lambda_{1} x}+e^{-\lambda_{2} x}+r\right)+t+u \cdot e^{-\frac{(x-v)^{2}}{2 w^{2}}} .
$$

To obtain an explicit description of the signal function, the following integrals have to be solved (for the sake of simplicity s $\sigma$ is replaced by $\sigma$ ):

$$
\begin{gathered}
\int_{-\infty}^{\infty} d y \theta(x-y) \cdot e^{\frac{-K(x-y)}{c \tau}} \cdot \frac{1}{\sigma \sqrt{2 \pi}} \cdot e^{\frac{-y^{2}}{2 \sigma^{2}}} \\
-\int_{-\infty}^{\infty} d y \theta(x-y) \cdot e^{\frac{-K(x-y)}{c \tau}} \cdot \frac{1}{\sigma \sqrt{2 \pi}} \cdot e^{\frac{-y^{2}}{2 \sigma^{2}}} \cdot y \\
\int_{-\infty}^{\infty} d y \theta(x-y) \cdot e^{\frac{-K(x-y)}{c \tau}} \cdot \frac{1}{\sigma \sqrt{2 \pi}} \cdot e^{\frac{-y^{2}}{2 \sigma^{2}}} \cdot e^{-\lambda_{1} K(x-y)} \\
-\int_{-\infty}^{\infty} d y \theta(x-y) \cdot e^{\frac{-K(x-y)}{c \tau}} \cdot \frac{1}{\sigma \sqrt{2 \pi}} \cdot e^{\frac{-y^{2}}{2 \sigma^{2}}} \cdot e^{-\lambda_{1} K(x-y)} \cdot y \\
\int_{-\infty}^{\infty} d y \theta(x-y) \cdot e^{\frac{-K(x-y)}{c \tau}} \cdot \frac{1}{\sigma \sqrt{2 \pi}} \cdot e^{\frac{-y^{2}}{2 \sigma^{2}}} \cdot e^{-\frac{(K(x-y)-v)^{2}}{2 w^{2}}}
\end{gathered}
$$


Solution for equation B.1:

$$
\begin{gathered}
\int_{-\infty}^{\infty} d y \theta(x-y) \cdot e^{\frac{-K(x-y)}{c \tau}} \cdot \frac{1}{\sigma \sqrt{2 \pi}} \cdot e^{\frac{-y^{2}}{2 \sigma^{2}}}= \\
\frac{1}{\sigma \sqrt{2 \pi}} \int_{-\infty}^{x} d y \cdot e^{\left[-\frac{1}{2 \sigma^{2}}\left(y^{2}-\frac{2 K \sigma^{2}}{c \tau} y\right)-\frac{K x}{c \tau}\right]}= \\
\frac{1}{\sigma \sqrt{2 \pi}} \int_{-\infty}^{x} d y \cdot e^{-\frac{1}{2 \sigma^{2}}\left(y-\frac{K \sigma^{2}}{c \tau}\right)^{2}} \cdot e^{\left[\frac{-K x}{c \tau}+\frac{K^{2} \sigma^{2}}{2 c^{2} \tau^{2}}\right]}= \\
\frac{1}{\sigma \sqrt{2 \pi}} \cdot e^{\left[\frac{-K x}{c \tau}+\frac{K^{2} \sigma^{2}}{2 c^{2} \tau^{2}}\right]} \cdot \int_{-\infty}^{x} d y \cdot e^{-\frac{1}{2}\left(\frac{y}{\sigma}-\frac{K \sigma}{c \tau}\right)^{2}}= \\
e^{\left[\frac{-K x}{c \tau}+\frac{K^{2} \sigma^{2}}{2 c^{2} \tau^{2}}\right]} \cdot \operatorname{freq}\left(\frac{x}{\sigma}-\frac{K \sigma}{c \tau}\right)
\end{gathered}
$$

with

$$
\operatorname{freq}(x)=\frac{1}{\sqrt{2 \pi}} \int_{-\infty}^{x} d t e^{-\frac{1}{2} t^{2}},
$$

which is also used to solve equations B.3 and B.5.

The solution of equations B.2 and B.4 uses the rule for partial integration:

$$
\int_{a}^{b} u v^{\prime}=[u v]_{a}^{b}-\int_{a}^{b} u^{\prime} v .
$$

Solution for equation B.2:

$$
\begin{gathered}
-\int_{-\infty}^{\infty} d y \theta(x-y) \cdot e^{\frac{-K(x-y)}{c \tau}} \cdot \frac{1}{\sigma \sqrt{2 \pi}} e^{\frac{-y^{2}}{2 \sigma^{2}}} \cdot y= \\
-\frac{1}{\sigma \sqrt{2 \pi}} \int_{-\infty}^{x} d y \underbrace{e^{\frac{-K(x-y)}{c \tau}}}_{u} \cdot \underbrace{y \cdot e^{\frac{-y^{2}}{2 \sigma^{2}}}}_{v^{\prime}}= \\
\frac{1}{\sigma \sqrt{2 \pi}}\left[e^{\frac{-K(x-y)}{c \tau}} \cdot \sigma^{2} \cdot e^{\frac{-y^{2}}{2 \sigma^{2}}}\right]_{-\infty}^{x}-\frac{1}{\sigma \sqrt{2 \pi}} \int_{-\infty}^{x} \frac{K}{c \tau} \cdot e^{\frac{-K(x-y)}{c \tau}} \cdot \sigma^{2} \cdot e^{-\frac{y^{2}}{2 \sigma^{2}}}= \\
\frac{\sigma}{\sqrt{2 \pi}} \cdot e^{-\frac{x^{2}}{2 \sigma^{2}}}-\frac{K \sigma^{2}}{c \tau} \cdot \text { equation B.1 }
\end{gathered}
$$


Solution for equation B.3:

$$
\begin{gathered}
\int_{-\infty}^{\infty} d y \theta(x-y) \cdot e^{\frac{-K(x-y)}{c \tau}} \cdot \frac{1}{\sigma \sqrt{2 \pi}} \cdot e^{\frac{-y^{2}}{2 \sigma^{2}}} \cdot e^{-\lambda_{1} K(x-y)}= \\
\frac{1}{\sigma \sqrt{2 \pi}} \int_{-\infty}^{x} d y e^{-\frac{1}{2 \sigma^{2}}\left[y^{2}-2 \sigma^{2}\left(\frac{K}{c \tau}+\lambda_{1} K\right) \cdot y\right]} \cdot e^{\left[-\frac{K x}{c \tau}-\lambda_{1} K x\right]}= \\
\frac{1}{\sigma \sqrt{2 \pi}} \int_{-\infty}^{x} d y e^{-\frac{1}{2}\left[\frac{y}{\sigma}-\sigma K\left(\frac{1}{c \tau}+\lambda_{1}\right)\right]^{2}} \cdot e^{\left[-K x\left(\frac{1}{c \tau}+\lambda_{1}\right)+\frac{\sigma^{2} K^{2}}{2}\left(\frac{1}{c \tau}+\lambda_{1}\right)^{2}\right]}= \\
e^{\left[-K x\left(\frac{1}{c \tau}+\lambda_{1}\right)+\frac{\sigma^{2} K^{2}}{2}\left(\frac{1}{c \tau}+\lambda_{1}\right)^{2}\right]} \cdot \text { freq }\left[\frac{x}{\sigma}-\sigma K\left(\frac{1}{c \tau}+\lambda_{1}\right)\right]
\end{gathered}
$$

Solution for equation B.4:

$$
\begin{gathered}
-\int_{-\infty}^{\infty} d y \theta(x-y) \cdot e^{\frac{-K(x-y)}{c \tau}} \cdot \frac{1}{\sigma \sqrt{2 \pi}} \cdot e^{\frac{-y^{2}}{2 \sigma^{2}}} \cdot e^{-\lambda_{1} K(x-y)} \cdot y= \\
-\frac{1}{\sigma \sqrt{2 \pi}} \int_{-\infty}^{x} d y \underbrace{e^{\frac{-K(x-y)}{c \tau}} \cdot e^{-\lambda_{1} K(x-y)}}_{u} \cdot \underbrace{y \cdot e^{-\frac{y^{2}}{2 \sigma^{2}}}}_{v^{\prime}}= \\
\frac{1}{\sigma \sqrt{2 \pi}} \cdot\left[e^{-K x\left(\frac{1}{c \tau}+\lambda_{1}\right)} \cdot e^{K y\left(\frac{1}{c \tau}+\lambda_{1}\right)} \cdot \sigma^{2} \cdot e^{-\frac{y^{2}}{2 \sigma^{2}}}\right]_{-\infty}^{x}- \\
\frac{1}{\sigma \sqrt{2 \pi}} \int_{-\infty}^{x} d y K\left(\frac{1}{c \tau}+\lambda_{1}\right) \cdot e^{\frac{-K(x-y)}{c \tau}} \cdot e^{-\lambda_{1} K(x-y)} \cdot \sigma^{2} \cdot e^{-\frac{y^{2}}{2 \sigma^{2}}}= \\
\frac{\sigma}{\sqrt{2 \pi}} \cdot e^{-\frac{x^{2}}{2 \sigma^{2}}}-K \sigma^{2}\left(\frac{1}{c \tau}+\lambda_{1}\right) \cdot \text { equation B.3 }
\end{gathered}
$$


Solution for equation B.5:

$$
\begin{aligned}
& \int_{-\infty}^{\infty} d y \theta(x-y) \cdot e^{\frac{-K(x-y)}{c \tau}} \cdot \frac{1}{\sigma \sqrt{2 \pi}} \cdot e^{\frac{-y^{2}}{\sigma^{2}}} \cdot e^{-\frac{(K(x-y)-v)^{2}}{2 w^{2}}}= \\
& \frac{1}{\sigma \sqrt{2 \pi}} \int_{-\infty}^{x} d y e^{\left[-\frac{K x}{c \tau}+\frac{K y}{c \tau}-\frac{y^{2}}{2 \sigma^{2}}-\frac{(K x-v-K y)^{2}}{2 w^{2}}\right]}= \\
& \frac{1}{\sigma \sqrt{2 \pi}} \int_{-\infty}^{x} d y e^{\left[-\frac{K x}{c \tau}-\frac{(K x-v)^{2}}{2 w^{2}}\right]} \cdot e^{\left[-\frac{1}{2}\left(\frac{1}{\sigma^{2}}+\frac{K^{2}}{w^{2}}\right) \cdot y^{2}+\left(\frac{K^{2} x}{w^{2}}+\frac{K}{c \tau}-\frac{v K}{w^{2}}\right) \cdot y\right]}=
\end{aligned}
$$

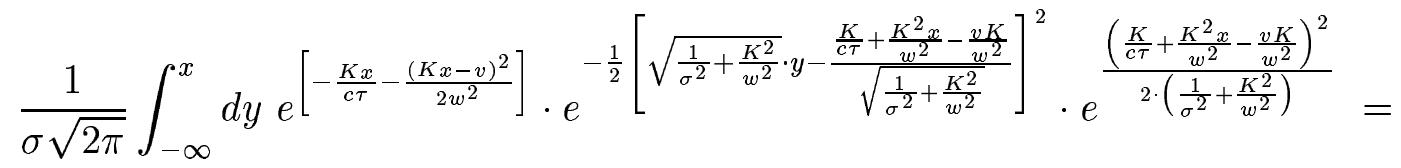

$$
\begin{aligned}
& \frac{1}{\sigma} e^{\left[-\frac{K x}{c \tau}-\frac{(K x-v)^{2}}{2 w^{2}}+\frac{\left(\frac{K}{c \tau}+\frac{K^{2} x}{w^{2}}-\frac{v K}{w^{2}}\right)^{2}}{2 \cdot\left(\frac{1}{\sigma^{2}}+\frac{K^{2}}{w^{2}}\right)}\right]} \cdot \text { freq }\left(\sqrt{\frac{1}{\sigma^{2}}+\frac{K^{2}}{w^{2}}} \cdot x-\frac{\frac{K}{c \tau}+\frac{K^{2} x}{w^{2}}-\frac{v K}{w^{2}}}{\sqrt{\frac{1}{\sigma^{2}}+\frac{K^{2}}{w^{2}}}}\right) \cdot \frac{1}{\sqrt{\frac{1}{\sigma^{2}}+\frac{K^{2}}{w^{2}}}}
\end{aligned}
$$

\section{B.2 Calculation of the Background Function}

The background function $\mathcal{F}_{b}$ is parametrised as a $\delta$-function plus an exponential with positive lifetime, which are again convoluted with a Gaussian resolution function:

$$
\mathcal{F}_{b}=(\delta+\exp ) \otimes G=\delta \otimes G+\exp \otimes G=f_{\delta}+f_{\exp } .
$$

The convolution of a $\delta$-function with a Gaussian yields a Gaussian:

$$
f \otimes \delta=\int f\left(x^{\prime}\right) \delta\left(x-x^{\prime}\right) d x^{\prime}=f(x)
$$

and the normalised contribution of the $\delta$ term to the fit function is:

$$
f_{\delta}=\frac{1}{\sqrt{2 \pi} \sigma_{0}} \cdot \exp \left[-0.5 \cdot\left(\frac{x-X_{0}}{\sigma_{0}}\right)^{2}\right] .
$$

The calculation of the convolution of an exponential with a Gaussian resolution function is shown below. 
Calculation of $f_{\text {exp }}$ :

$$
\begin{gathered}
f_{\exp }=\exp \otimes G=\frac{1}{\sqrt{2 \pi} \sigma_{b}} \int_{-\infty}^{\infty} d y \theta(y) \cdot e^{-\frac{y}{c \tau_{b}}} \cdot e^{-\frac{1}{2} \frac{(x-y)^{2}}{\sigma_{b}^{2}}}= \\
\frac{1}{\sqrt{2 \pi} \sigma_{b}} \int_{0}^{\infty} d y e^{-\frac{y}{c \tau_{b}}} \cdot e^{\left[-\frac{x^{2}}{2 \sigma_{b}^{2}}-\frac{y^{2}}{2 \sigma_{b}^{2}}+\frac{x y}{\sigma_{b}^{2}}\right]}= \\
\frac{1}{\sqrt{2 \pi} \sigma_{b}} \int_{0}^{\infty} d y e^{-\frac{1}{2 \sigma_{b}^{2}}\left[y^{2}+\left(\frac{2 \sigma_{b}^{2}}{c \tau_{b}}-2 x\right) \cdot y\right]} \cdot e^{-\frac{x^{2}}{2 \sigma_{b}^{2}}}= \\
\frac{1}{\sqrt{2 \pi} \sigma_{b}} \int_{0}^{\infty} d y e^{-\frac{1}{2}\left(\frac{y}{\sigma_{b}}+\frac{\sigma_{b}}{c \tau_{b}}-\frac{x}{\sigma_{b}}\right)^{2}} \cdot e^{\left[\frac{1}{2}\left(\frac{\sigma_{b}}{\tau_{b}}\right)^{2}-\frac{x}{c \tau_{b}}\right]}= \\
\frac{1}{\sqrt{2 \pi} \sigma_{b}} \cdot e^{\left[\frac{1}{2}\left(\frac{\sigma_{b}}{c \tau_{b}}\right)^{2}-\frac{x}{c \tau_{b}}\right]} \cdot\left[1-\int_{-\infty}^{0} d y e^{\left.-\frac{1}{2}\left(\frac{y}{\sigma_{b}}+\frac{\sigma_{b}}{c \tau_{b}}-\frac{x}{\sigma_{b}}\right)^{2}\right]}=\right. \\
e^{\left[\frac{1}{2}\left(\frac{\sigma_{b}}{c \tau_{b}}\right)^{2}-\frac{x}{c \tau_{b}}\right]} \cdot\left[1-\text { freq }\left(\frac{\sigma_{b}}{c \tau_{b}}-\frac{x}{\sigma_{b}}\right)\right]
\end{gathered}
$$

The normalisation of $f_{\exp }$ yields an additional factor of $1 / c \tau_{b}$, and $x$ is replaced by $x-X_{b}$ to include a shift. This yields for $f_{\exp }$ the result quoted in section 5.2.2:

$$
f_{\exp }=\frac{1}{c \tau_{b}} \cdot \exp \left[\frac{1}{2}\left(\frac{\sigma_{b}}{c \tau_{b}}\right)^{2}-\frac{x-X_{b}}{c \tau_{b}}\right] \cdot\left[1-\operatorname{freq}\left(\frac{\sigma_{b}}{c \tau_{b}}-\frac{x-X_{b}}{\sigma_{b}}\right)\right] .
$$




\section{Bibliography}

[1] K. Hagiwara et al. Review of Particle Physics. Physical Review, D66:010001, 2002.

[2] H. Weyl. A new extension of Relativity Theory. (in German). Annalen Phys., 59:101-133, 1919.

[3] Gerard 't Hooft. Renormalization of massless Yang-Mills fields. Nucl. Phys., B(33):173-199, 1971.

[4] Gerard 't Hooft. Renormalizable Lagrangians for massive Yang-Mills fields. Nucl. Phys., B(35):167-188, 1971.

[5] Peter W. Higgs. Broken Symmetries, Massless Particles and Gauge Fields. Phys. Lett., 12:132-133, 1964.

[6] Peter W. Higgs. Broken Symmetries and the Masses of Gauge Bosons. Phys. Rev. Lett., 13:508-509, 1964.

[7] F. Englert and R. Brout. Broken Symmetry and the Mass of Gauge Vector Mesons. Phys. Rev. Lett., 13:321-322, 1964.

[8] G.S. Guralnik, C.R. Hagen, and T.W.B. Kibble. Global Conservation Laws and Massless Particles. Phys. Rev. Lett., 13:585-587, 1964.

[9] T.W.B. Kibble. Symmetry Breaking in Nonabelian Gauge Theories. Phys. Rev., 155:1554-1561, 1967.

[10] J.F. Gunion, H.E. Haber, G.L. Kane, and S. Dawson. The Higgs Hunter's Guide. Perseus Publishing, Cambridge, Massachusetts, 2000. 
[11] E. Noether. Invariante Variationsprobleme. Nachr. v. d. Ges. d. Wiss zu Göttingen, pages 235-257, 1918.

[12] T.D. Lee and Chen-Ning Yang. Question of Parity Conservation in Weak Interactions. Phys. Rev., 104:254-258, 1956.

[13] C.S. Wu, E. Ambler, R.W. Hayward, D.D. Hoppes, and R.P. Hudson. Experimental test of parity conservation in beta decay. Phys. Rev., 105:1413-1414, 1957.

[14] CPLEAR Collaboration (A. Angelopoulos et al.). First direct observation of time-reversal non-invariance in the neutral kaon system. Phys. Lett., B(444):43$51,1998$.

[15] Michelangelo L. Mangano. Two Lectures on Heavy Quark Production in Hadronic Collisions. CERN-TH/97-328, hep-ph/9711337.

[16] CDF Collaboration (T. Affolder et al.). Measurement of the $b$ Quark Fragmentation Fractions in $p \bar{p}$ Collisions at $\sqrt{s}=1.8 \mathrm{GeV}$. Phys. Rev. Lett., 84:1663-1668, 2000 .

[17] C. Peterson, D. Schlatter, I. Schmitt, and Peter M. Zerwas. Scaling Violations in inclusive $e^{+} e^{-}$Annihilation Spectra. Phys. Rev., D(27):105, 1983.

[18] The ALEPH Collaboration (D. Buskulic et al.). Strange b baryon production and lifetime in Z decays. Phys. Lett., B(384):449-460, 1996.

[19] DELPHI Collaboration (P. Abreu et al.). Production of strange B baryons decaying into $\Xi^{\mp}-l^{\mp}$ pairs at LEP. Z. Phys., C(68):541-554, 1995.

[20] Gerhard Buchalla. Heavy Quark Theory. CERN-TH/2002-018, hepph/0202092.

[21] M. Battaglia, A.J. Buras, P. Gambino, and A. Stocchi [eds.]. The CKM Matrix and the Unitarity Triangle: Proceedings based on the Workshop held at CERN, 13 - 16 Feb 2002. CERN-EP/2003-002,hep-ph/0304132, 2003. 
[22] S. Eidelman et al. (Particle Data Group). The Review of Particle Physics. Phys. Lett., B 592:1, 2004.

[23] Ronald S. Moore. An Overview of Tevatron Collider Run II at Fermilab. FERMILAB-CONF-02-320-E, 2002.

[24] Edward Wilson. An Introduction to Particle Accelerators. Oxford University Press, 2001. Name on book cover wrongly given as Edmund.

[25] The CDF II Collaboration. The CDF II Detector Technical Design Report. FERMILAB-Pub-96/390-E.

[26] The CDF II Collaboration. Proposal for Enhancement of the CDF II Detector: An Inner Silicon Layer and A Time of Flight Detector. CDF Note 5096, October 1998. Submitted to the PAC.

[27] M. Garcia-Sciveres, O. Milgrome, T. Zimmerman, I. Volobuev, R. P. Ely, A. Connolly, D. Fish, T. Affolder, and A. Sill. The SVX3D integrated circuit for dead-timeless silicon strip readout. Nucl. Instr. Meth., A(435):58-64, 1999.

[28] Alan Sill for the CDF Collaboration. CDF Run II silicon tracking projects. Nucl. Instr. Meth., A(447):1-8, 2000.

[29] D. Acoste et al. Measurement of the $J / \psi$ Meson and b Hadron Production Cross Sections in $p \bar{p}$ Collisions at $\sqrt{s}=1960 \mathrm{GeV}$. CDF Note 7037, 2005. FERMILABPub-04-440-E.

[30] K. Anikeev et al. B Physics at the Tevatron: Run II and Beyond. FERMILABPub-01/197,hep-ph/0201071.

[31] S. Cabrera, I. Cho, D.H. Kim, Y. Oh, J. Piedra, T. Rodrigo, A. Ruiz, I. Vila, and I. Yu. Tests and calibration of the photomultipliers of the CDF-II Timeof-Flight system. CDF Note 5386, November 2000.

[32] S. Cabrera et al. The CDF-II Time-of-Flight Detector. CDF Note 6258, 2003. 
[33] N. Ellis and J. March-Russel. 1998 European School of High-Energy Physics. CERN 99-04, CERN, July 1999.

[34] R. Blair, S. Kuhlmann, L. Nodulman, J. Proudfoot, and A.B. Wicklund. Proposal for a Preradiator Chamber for the CDF Central Detector. CDF Note 760, Argonne National Laboratory, Argonne, IL.

[35] T. Kamon, K. Kondo, A. Yamashita, T. Shimizu, and L. Nodulman. A New Scintillator and Wavelength Shifter. Nucl. Instr. Meth., (213):261, 1983.

[36] The CDF Collaboration (L. Balka et al.). The CDF Central Electromagnetic Calorimeter. Nucl. Instr. Meth., A(267):272, 1988.

[37] Ryutaro Oishi on behalf of the CDF Plug Upgrade Group. New CDF end-plug Calorimeter. Nucl. Instr. Meth., A(453):227-229, 2000.

[38] Gerry Bauer, Jerome Friedman, Elizabeth Hafen, Kevin McFarland, Lawrence Rosenson, Paris Sphicas, Sham Sumorok, Steve Tether, Kirsten Tollefson, and Jeff Tseng. Proposal for Expansion of the Event-Builder and Level-3 Trigger to a $1000 \mathrm{~Hz}$ Input Rate. CDF Note 4623, 1998.

[39] Mike Kirk, Jonathan Lewis, and Masashi Tanaka. Run II Semileptonic Trigger Study. CDF Note 5324, November 2000.

[40] Aseet Mukherjee. CTC and VTX Tracking. CDF Note 5490, 2000.

[41] Paolo Gatti. Performance of the new tracking system at CDF II. CDF Note 5561, December 2000.

[42] S. Menzemer. TrackingKal - A Tracking and Alignment Software Package for the CDFII Silicon Detector. CDF Note 5968, 2002.

[43] J. Marriner. SECONDARY VERTEX FIT WITH MASS AND POINTING CONSTRAINTS (CTVMFT). CDF Note 1996, 1993.

[44] W. R. Leo. Techniques for Nuclear and Particle Physics Experiments. SpringerVerlag, 1994. 
[45] Shin-Shan Yu, Joel Heinrich, Nigel Lockyer, Dave Ambrose, and Peter Wittich. COT dE/dx Measurement and Corrections. CDF Note 6361, 2003.

[46] Saverio D'Auria et al. Track-based calibration of the COT specific ionization. CDF Note 6932, April 2004.

[47] H.-C. Fang, A. Cerri, M.D. Shapiro, M. Tanaka, and S. Uozumi. SemiLeptonicB/LeptonSvtSel- An offline filter module for lepton-SVT data. CDF Note 6326, February 2003.

[48] K. Anikeev, P. Murat, and Ch. Paus. Description of Bgenerator II. CDF Note 5092, 1999.

[49] Jonathan Lewis and Paul Avery. CLEOMC: The CDF Interface to the CLEO Monte Carlo (QQ). CDF Note 2724, 1994.

[50] Manfred Paulini. CDF Detector Simulation. http://wwwcdf.fnal.gov/cdfsim/cdfsim_main.html.

[51] S. Uozumi. Study of Trigger Bias to B Lifetimes. CDF Note 6321, 2003.

[52] J. Tseng, B. Barnett, J. Skarha, and T. Miao. Observation of Bottom Baryon Semileptonic Decay. CDF Note 3353, 1995.

[53] CLEO Collaboration (D. Cinabro et al.). A Study of Baryon-Production in B-decay: Search for Semileptonic decays of $B$ mesons to Charmed Baryons and the First Observation of $\Xi_{c}$ Production in B-decay. CLEO CONF 94-8, 1994.

[54] R.K. Ellis, W.J. Stirling, and B.R. Webber. QCD and Collider Physics. Cambridge University Press, 1996.

[55] The CDF Collaboration (F. Abe et al.). Measurement of $\Lambda_{b}^{0}$ Lifetime Using $\Lambda_{b}^{0} \rightarrow \Lambda_{C}^{+} l^{-} \bar{\nu}$. Phys. Rev. Lett., 77:1439-1443, 1996.

[56] The OPAL Collaboration (K. Ackerstaff et al.). Measurements of the $\mathrm{B}_{\mathrm{s}}^{0}$ and $\Lambda_{\mathrm{b}}^{0}$ Lifetimes. Phys. Lett., B(426):161-179, 1998.

[57] Masashi Tanaka. Personal communication. 
[58] M. Tanaka. Semileptonic B group meeting, 6th April 2004.

[59] M. Tanaka. Semileptonic B group meeting, 18th February 2003.

[60] F. James. MINUIT - Function Minimization and Error Analysis. CERN Program Library Long Writeup D506, 1998.

[61] The input samples for the blind lifetime fit test were provided by Masashi Tanaka.

[62] P.S. Wells. Systematic variation of fragmentation parameters for $\mathrm{b}$ physics analyses. OPAL Technical Note TN125, 1996.

[63] ALEPH Collaboration (D. Buskulic et al.). Measurement of the effective b quark fragmentation function at the $\mathrm{Z}$ resonance. Phys. Lett., B(357):699-714, 1995.

[64] The ALEPH Collaboration (D. Buskulic et al.). Measurement of the $\Lambda_{b}$ polarization in Z decays. Phys. Lett., B(365):437-447, 1996.

[65] The DELPHI Collaboration (P. Abreu et al.). $\Lambda_{b}$ Polarization in $Z^{0}$ decays at LEP. Phys. Lett., B(474):205-222, 2000.

[66] The OPAL Collaboration (G. Abbiendi et al.). Measurement of the Average Polarization of b Baryons in Hadronic $Z^{0}$ Decays. Phys. Lett., B(444):539-554, 1998.

[67] LEP B lifetimes working group. Averages of B Hadron Lifetimes for PDG 2004. http://lepbosc.web.cern.ch/LEPBOSC/lifetimes/lepblife_page2.html.

[68] The ALEPH Collaboration (D. Barate et al.). Measurement of the b baryon lifetime and branching fractions in Z decays. Eur. Phys. J., C(2):197-211, 1998.

[69] DELPHI Collaboration (P. Abreu et al.). Measurement of the Lifetime of bbaryons. Eur. Phys. J., C(10):185-199, 1999.

[70] Ikaros I. Y. Bigi, B. Blok, Mikhail A. Shifman, N. Uraltsev, and Arkady I. Vainshtein. Nonleptonic decays of beauty hadrons: From phenomenology to theory. 1994. hep-ph/9401298. 